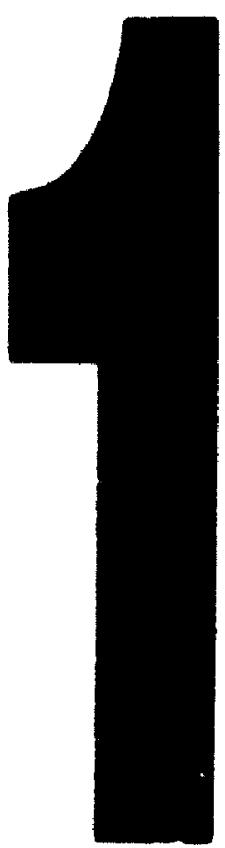

PM-1 3","x4" PHOTOCRAPHIC MICROCOPY TARGET NAS 1010 ANSI/ISO *2 EOUIVALENT

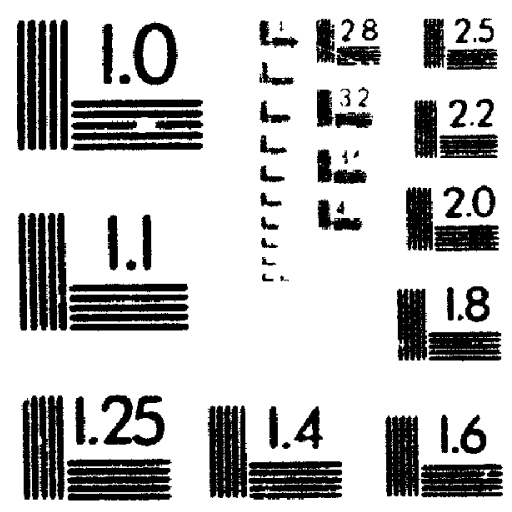

PRECISION"m RESOLUTION TARGETS 


\title{
GEOMETRIC DESIGN CONSIDERATIONS OF COMBINED HORIZONTAL AND VERTICAL HIGHWAY ALIGNMENTS
}

\author{
by \\ YASSER HASSAN \\ B.Sc.. Cairo University. Egypt \\ M.Sc., Cairo University. Egynt \\ A thesis submitted to \\ the Faculty of Graduate Studies and Research \\ in partial fulfilment of \\ the requirements of the degree of \\ Doctor of Philosophy \\ in Civil Engineering \\ Department of Civil and Environmental Engineering \\ Carleton University \\ Ottawa. Ontario, Canada \\ (1) copyright \\ 1996. Yasser Hassan
}

The Doctor of Philosophy in Civil Engineering

is a joint program with the University of Ottawa

administered by the Ottawa-Carleton Institute for Civil Engineering 
The author has granted an irrevocable non-exclusive licence allowing the National Library of Canada to reproduce, loan, distribute or sell copies of his/her thesis by any means and in any form or format, making this thesis available to interested persons.
L'auteur a accordé une licence irrévocable et non exclusive permettant à la Bibliothèque nationale du Canada de reproduire, prêter, distribuer ou vendre des copies de sa thèse de quelque manière et sous quelque forme que ce soit pour mettre des exemplaires de cette thèse à la disposition des personnes intéressées.

L'auteur conserve la propriété du droit d'auteur qui protège sa thèse. Ni la thèse ni des extraits substantiels de celle-ci ne doivent être imprimés ou autrement reproduits sans son autorisation.

ISBN $\quad 0-612-19377-2$ 
Nom Jasser Hassan

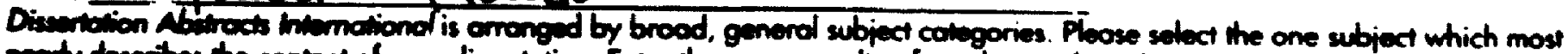
nocity deveribes the combent of your disemtation. Emer the corrosponding four-digit cade in the spoces provided.

Civil Engineering $015[413 \mathrm{U} \cdot \mathrm{M} \cdot \mathrm{I}$ Sulipa Compories

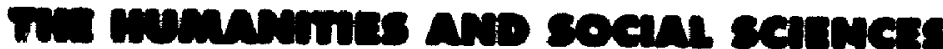

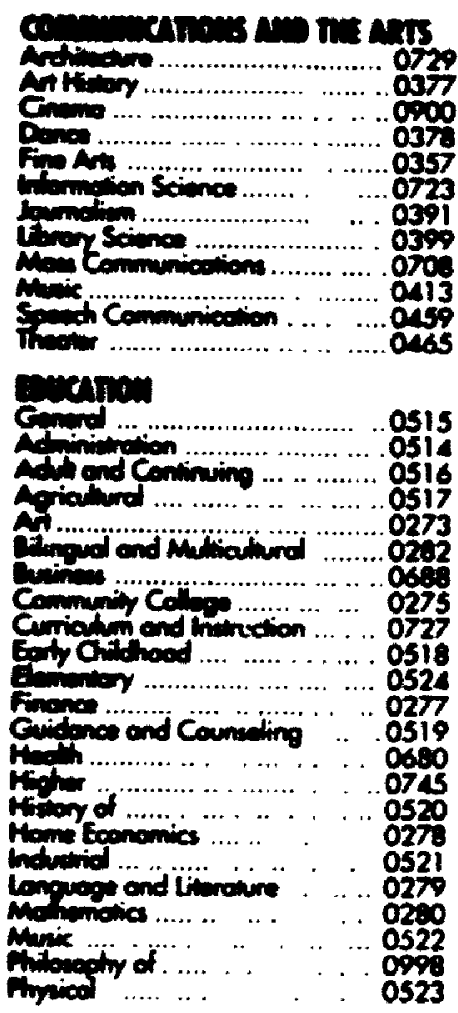

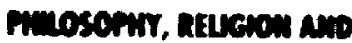

Thivesr

Philosophy

Religen

Genord

Stucel Studves

Cligror

Pitory of

Theolegy

secil seinas

Anericon Studies

Antiropelogy

Ancheodogy

Cenunge

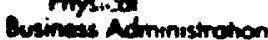

Geverd

Accouning

Bonting

Monogoment

Mortong

Comodion Stidies

Economins:

Gomerol

Connerimed

Finonce

lobor

Theory

Georlore

Geromology

History

Gonerd
Commerce Busness
0422

0318

0321

0310

0320

0469

0323

032

0326

0327

0310

0272

0770

0454

0338
0385

0sol

0503

0505

0508

0510

O5II

0358

0366

0351

0578

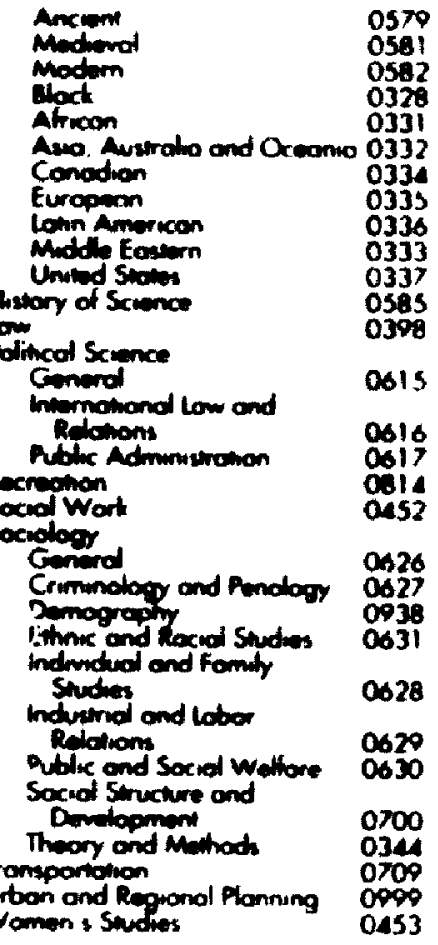

0460

0383

home Economics

intricu satass

Pur Scinces

Chemingy

Agriculural

Anodticel

Brachomisty

morgonic

Nucinor

Promocantice

Aingencel

Polymer

Rodiction

Mothemoncs

Prysics

Gaverd

Aderenomy and

Atroptraces

Amospherc Science

Alomic

Electronics and Electricty Elementory Pandes and

Aut Energy

fhid ond Phosmo

Moleculor

Nudier

Opties

Rotiction

Solud Stow

Sinomitics

Anctiod Seineses

Aplund Machonic:

Compuier serence
0386

0485

0749

0486

0488

0738

049

0,91

0.49

0495

0405

0605

0986

0006

0608

0748

0607

0798

0759

0609

0610

Q611

0.463

0346

0984
Engineering

Aerospoce

Agrouthrol

Aulometive

Chemical

Civel

Electronucs and Elactircol

Hydrouke

industrina

Morine

Movernots Sxience

Machonted

Monolwergr

Mining

Aucher

Poctioging

Petroleim

Sontion and Municipal

System Science

Opemonoms Reverch

Plosucs Technology

Iexibe Technology

ricuger

Conerol

chavioral

Chrucel

Demelopmantel

Espermenter

induenriog.

Provondiny

Piychebialogr

Pypctiomatric:

Sociol
Heot and thermodymomes

Geovechnology $\cos 37$

0536

0539

O5.

054

0543

0344

OS4S

0546

0547

Or94

074

05. 1

055:

0540

0765

$05=$

0428

0796

0795

0994

- 21

0384

022

0623

Os24

0025

0349

0632

0.451 
The undersigned hereby recommend to the Faculty of Graduate Studics and Research acceptance of the thesis,

\title{
Geometric Design Considerations of Combined
} Horizontal and Vertical Highway Alignments

\author{
submitted hy
}

Mr. Yasser Hassan, B.Sc., M.Sc.

in partial fulfilment of the requirements for the degree of Doctor of Philosophy

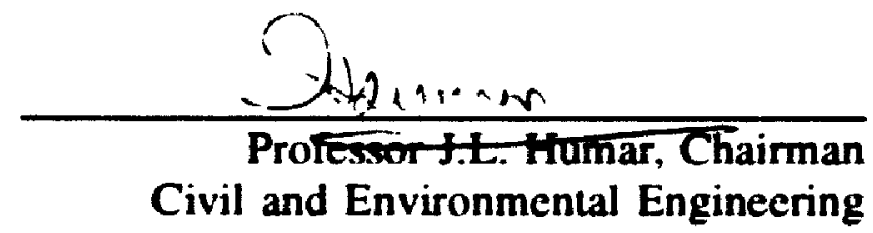

Said M. Huy

for. Professor A.O. Abd El Halim. Co-supervisor Civil and Environmental Engineering

$$
\text { - Said m. Hy }
$$

Professor S. Easa, Co-supervisor Civil Engineering, Lakehead University

$$
\text { Xt incuale }
$$

Professor J. Morrall, External Examiner Civil Engineering. The University of Calgary

Carleton University 


\section{ABSTRACT}

The process of highway design is a complex one where many phases are incorporated to achieve safe. efficient, conomical, and aesthetically pleasant highways. Among these phases. geometric design is the most related to traffic salety. Highwaly efficiency, economics. and aesthetics depend on the decisions taken in the geometric destgn phase. Thus. design standards have been set to guarantee the achievement of the design objectives. However. these standards are based on 2-D modelling of highwatys where the horizontal alignment. vertical alignment. and cross-section are designed separately.

In this research. a framework is presented for 3-D combined highway alignracnts to jointly design all highway elements based on sight distance, vehicle vtability. driver comfort. drainage, and aesthetics. Specifically, this research focuses on the daytime sight distance and its related aspects. First, a revised model for the required passing sight distance and minimum length of passing zones is developed, and the resulting passing sight distances show good agreement with field measurements. Second, a new concept for positioning the beginning and end of passing zones is presented and modelled analytically. Third. the available sight distance on complex 2-D horizontal and vertical alignments and 3-D alignments is modeiled. Computer software are developed hased on these analytical models, and each software is verified graphically or hy field measurements. Applications of the developed models and software are presented in markin" and design. 
The application of the developed models for the required passing sight distance show the need for revismom in the marking standards to ensure satety and efficiency of the trallic operation. The model for available sight distance provide a useful tool to replace the current graphical and field practees to estahlos the no-passing rones on twolane highways. Such a toml would avond the potential human errors, reduce the cost. and minimise the ume required for marking. A comparison with the current design standards hows lhetr inadequacy whether the highway is in cut or fill. The standards may compromise highway economics or traffic safety. As a result. the need for revisions in the destgn slandards based on 3-D alignments is established. 


\section{ACKNOWLEDGMENTS}

The author is grateful to William Hughes. Transportamen and Howh Wepatment.

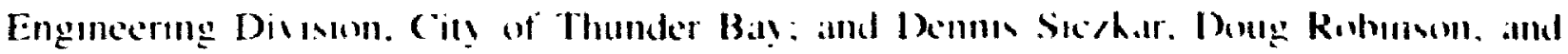

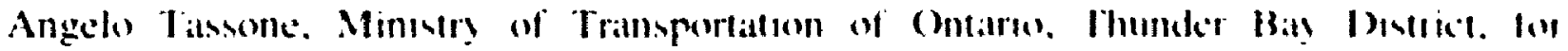

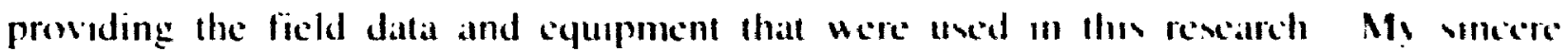
gratitude to Karen Williams. Lathehead l'niterstly. Thunder Hay. for her aswance in producing this thess: Kailash Bhatia and Ahmed Abo Talch. I.athehead I misersly.

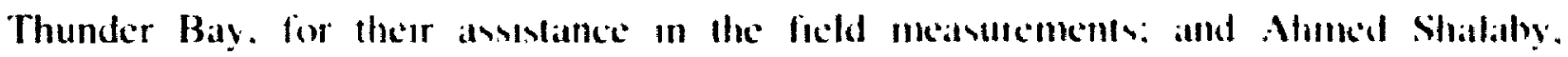
Carleton Insersity. Otlau a, for his continuous help for almost thec years The fmancial

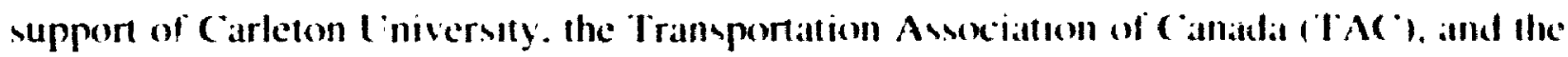
Natural Sa:ences and Engineering Research Council of Callada (NSI:RC i al ab glatlefully acknowledged.

Finallg. I would like to express my deepest gratllude fo I )r. Salld lialsat. I.atheheind Chiversity. Thunder Bay, and Dr. A.O. Abd El Halim, Carleton I iniverstly, (Mlawa, Ior their guidance. suggestions, help. support. and patience in supervistng this llesis. 


\section{TABIE OF CONTENTS}

ABSTRAC"T

iii

AC KNOWI.FIXIMENTS

$\mathbf{v}$

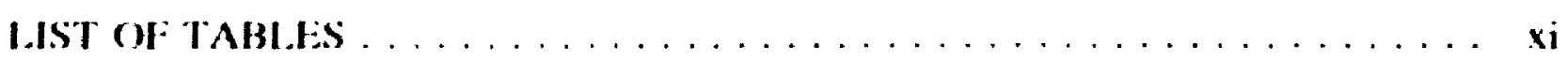

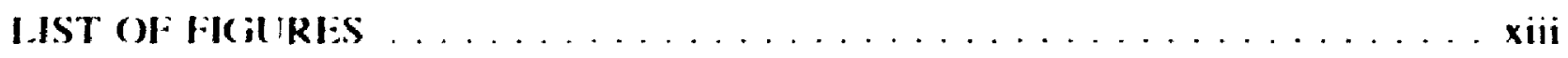

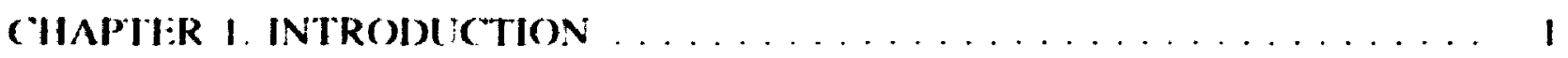

1.1 Highway Design Proress . . . . . . . . . . . . . . . 3

1.2 Present Status of Geometric Design . . . . . . . . . . . . 5

I.3 Problem Definitıon $\ldots \ldots \ldots \ldots \ldots \ldots$

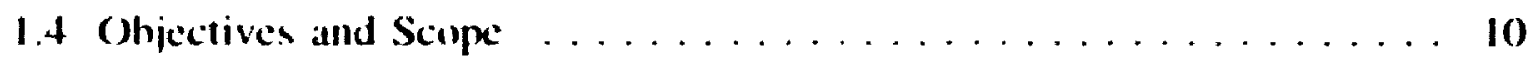

CIIAPTIE 2. REVIEW OF CIIRRENT DESIGN PRACTICE AND

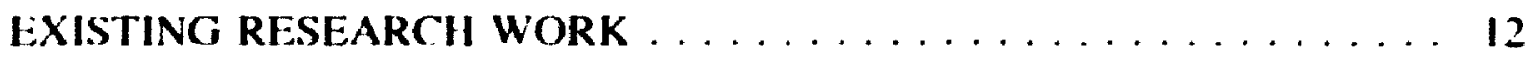

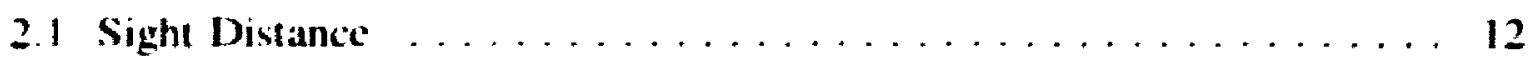

2.1.I Required Stopping Sight Distance $(S S D) \ldots \ldots \ldots \ldots$

2.1.2 Required Decision Sight Distance (DSD) .......... 17

2.1.3 Required Passing Sight Distance (PSD) . . . . . . . . 17

2.1 .4 Minimum Length of Passing Zones . . . . . . . . . . . 25

2.1 .5 Available Sight Distance ............... 27

2.2 Vehicle Stability and Driver Comfort $\ldots \ldots \ldots \ldots \ldots$

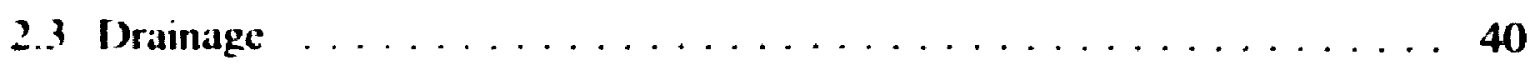

2.4 Highway Acsthetics . . . . . . . . . . . . . . 45 
2.5 Summary of Revicw

CHAPTER ? MODELLINÉ; REQQ'IRED PASSING SIGHT ISISINCI: ANI)

PASSINC; ZCINES .

3.1 ('ritique of Existing Models for PSI) . . . . . . . . . . . . . . . . .

3.1 .1 Glennon's Madel .................... S1)

3.1 .2 Rillet el al + Model $\ldots \ldots \ldots \ldots \ldots \ldots$

3.2 Mechanism of the Passing Manoxuvre ............. . 5o

3.3 Critical Posituon and Critical Sight Distance $\ldots \ldots \ldots \ldots \ldots \ldots$. $\ldots \ldots$

3.4 Minimum Length of Passing Zono $\ldots \ldots \ldots \ldots \ldots$

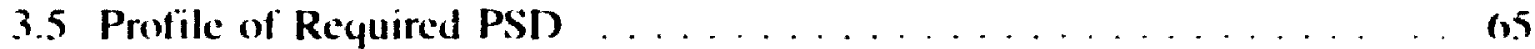

3.6 Model Simplification . . . . . . . . . . . . . . . . 70

3.7 Selection of Model Parameter $\ldots \ldots \ldots \ldots \ldots \ldots \ldots$

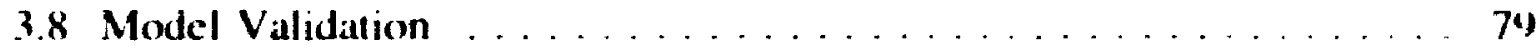

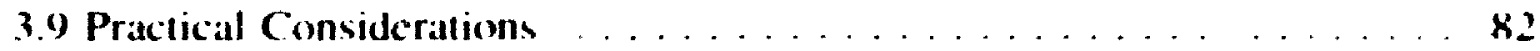

3.9.1 Design Values for Required PSI . . . . . . . . . . . . . 82

3.9.2 Design Values for Minimum Length of Passing Zomes . . . . 83

3.9.3 Beginning and End of Passing rones . . . . . . . . . 44

CHAPTER 1. AVAILABLE SIGHT DISTANCI: ON 2-I) SIPARATL:

ALIGNMENTS $\ldots \ldots \ldots \ldots \ldots \ldots \ldots \ldots \ldots \ldots \ldots$ k8

4.1 Horizontal Alignment with Continuous Obstruction . . . . . . . . . 8 8

4.1.1 General Procedure: Sight Line Tangent to ("ircular (arve ... x)

4.1.2 General Procedure: Sight Line Tangent to Spiral Curve .... M1 
41.3 Cieneral Procedure: Sight Line Passing Through Point of Intersection 96

414 Special case: Simple Circular Curve . . . . . . . . . . 97

4.1.5 Special casc: Intersecting Long Straight Segments . . . . . 101

4.2 Horizontal Alignment with Single Ohstructions $\ldots \ldots \ldots \ldots$

4.3 Vertical Alignment .................... 10.3

4.3.1 Cicometric Characteristics of Vertical Alignment Elements . 104

$\$ .3 .2$ Road as Sight Obstructiont . . . . . . . . . . . . I!)8

4.3 .3 Sight-Hidden Dips ................... 109

4.3 .4 Overpasces as Sight Ohstructions ............. 111

44 Initied Methodology for Separate Horizontal and Venical

Alignments ...................... 112

4.4.1 Background to Parametric Element: . . . . . . . . . . 113

4.4 .2 Alignment ldeatization $\ldots \ldots \ldots \ldots \ldots \ldots$

4.4.3 Intersection Between Sight Line and Parametric Elemerts. . 119

4.4.4 Iterative Procedure for Available Sight Distance . . . . 122

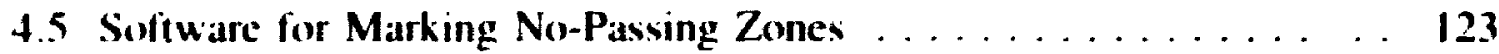

4.5 .1 Description of Developed Software $\ldots \ldots \ldots 123$

4.5.2 Software Verification $\ldots \ldots \ldots \ldots \ldots \ldots \ldots \ldots \ldots \ldots$

CHAPTER 5. AVAII ABLE SIGHT DISTANCE ON 3-D COMBINED

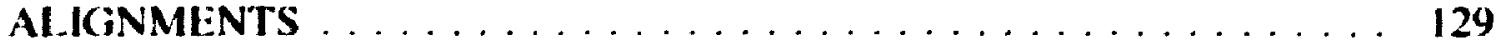

5.1 Elements in 3-D Alignment $\ldots \ldots \ldots \ldots \ldots \ldots \ldots$ 
5.2 Alignment Lealuatom into Parametric t:lements

5.3 Mathemattual Modelling

5.3.1 The 4-Node Rectangutar F:lement

5.3 .2 The 6-Node Recrangular Flemem

5.3.3 The 8-Node Rectangular I:lement

5.3.4 The 3-Node Triangular Flement

5.3.5 The 5-Node Triangular Element .

5.4 Itcrance Procedure for Avalahle Sight Distance

155

5.5 Softuare tor Marking No Passing Zomer

150

5.5.1 Dexcripton of Developed Softuare

1.50

5.5.2 Verification

I(x)

CHAPTER 6. (TTILIZATION OF DEVIALOPHAD MOIDIIS IN MARKINC;

TWO-LANE HIGHWAYS

6. I Alignment Data

6.2 Applicalion Objectives and Procedure

176

6.2.1 Applacation Objedtres . . . . . . . . . . . . . 176

6.2.2 Computer Programs and l ser-Specified Parameters

6.2.3 Experimental Design

6.3 Results and Analysis

6.3.1 Phase 1: Effect of Element Size

6.3.2 Phase 2: 2-D Versus 3-1) Sight Distances

I86

6.3.3 Phase 3: Comparison with Existing Marking in the Ficeld

I(x) 
6 3.4 Phase 4. Marking I sing Profiles of Avalable and

0.t Summary

( IIAPII:R 7. ISTABISISIMENT OI IOFSIGN PROVISIONS FOR 3-D

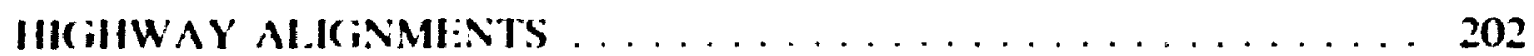

71 (urrent and $3-1$ ) Design Practices . . . . . . . . . 202

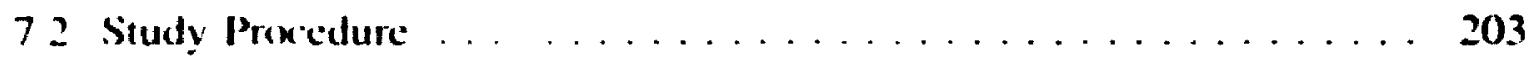

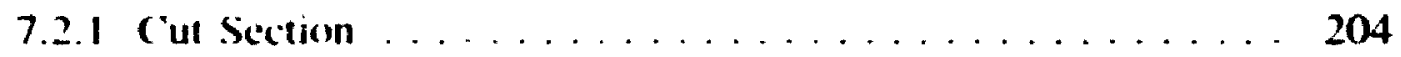

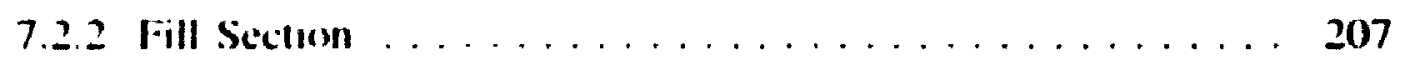

$7 \ldots$ Kesults and Analy is ..................... 208

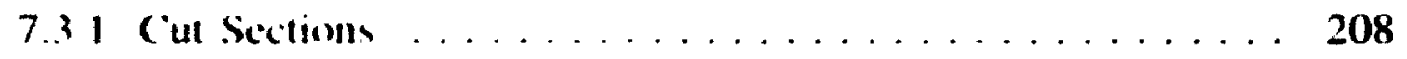

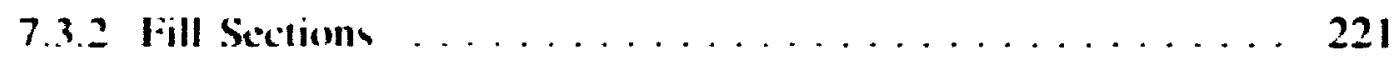

7.4 Summary ......................... 228

('HAPTIK \&. CONCLI'SIONS AND RECOMMENDATIONS . . . . . . . 230

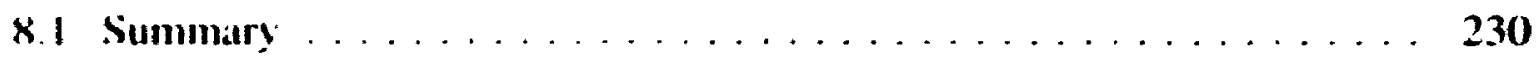

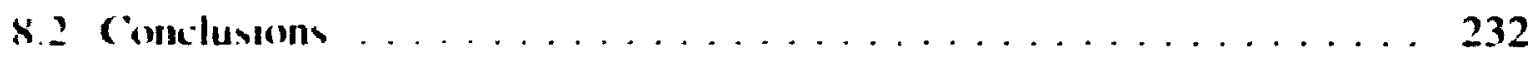

x.3 Recommendations and Future Research . . . . . . . . . 235

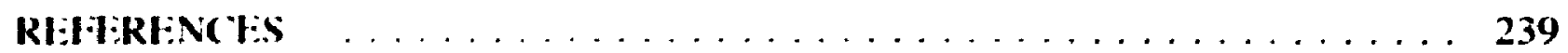




\section{I.IST OF TABLES}

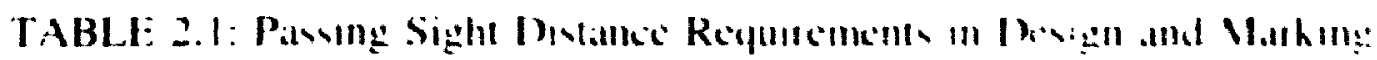

Standards in Candula.

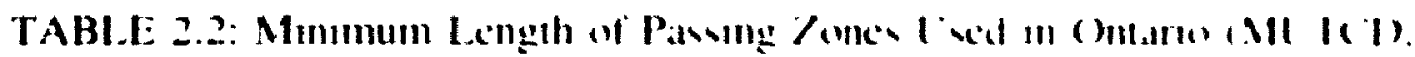
10051.

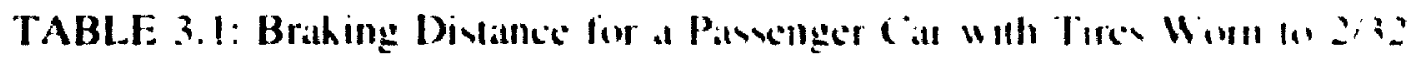

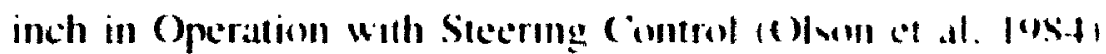

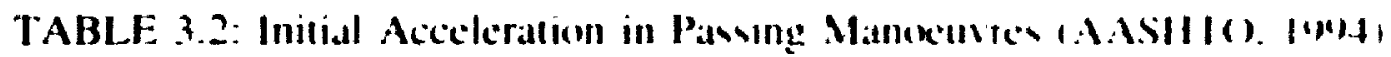

TABLE 4.: : Sample of Meisured and Calculated Vialtae ol I I Matlahle

Sight Distance.

TABLE 5.1: Measured and Cakulated Values of the Alaldale Sight Instance

167

TABLE 5.2: 's, sults of the Modified Vertical Alignment

$16 x$

TABLE 5.3: Tes of Hypothews for Avalabic Sight J'stiance

In)

TABLE 6.1: Summary of Fxpermental Ievgn.

TABLE 6.2: Effect of Element Size.

$|x|$

TABLE 6.3: Marking (sing Profile of Avallable PSI) and M! "T( 1$)(13,5$,

Standards.

19.8

TABLE 6.4: Segment Marking in the Ficld.

115

TABLE 6.5: Marking ('ung Profiles of Keyuired and 2-D Avalable (PSI)

147

TABLE 6.6: Marking (lsong Profiles of Required and 2.1) Avalaible PSil)

lox 
TABLE 7.1: Mininum Horizontal Curve Radius, $R$, and Design Values of $K$ According to Current Design Practice. . . . . . . . . . . . . . 209

TABLE 7.2: Difference Between 2-D and 3-D Radii for Horizontal Curves

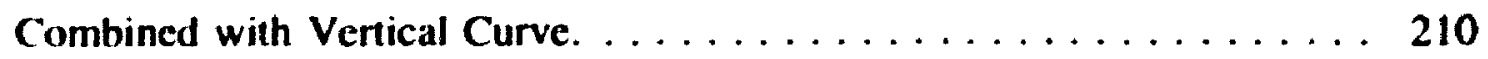

TABLE 7.3: 2-D and 3-D Values of $R$ for Separate and Long Horizontal Curves in Cut Sections $\left(A=0, L_{1}>\right.$ SSD $) \ldots \ldots \ldots \ldots \ldots \ldots \ldots \ldots$

TABLE 7.4: Effect of Vertical Curvature, Superelevation Rate, and Side-Slope on 3-D $R$

TABLE 7.5: Effect of Down-Slope and Drainage Ditch Before Up-Slope on 3-

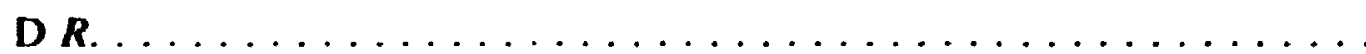

TABLE 7.6: Minimum $\boldsymbol{R}$ for Horizontal Curve Considering the Superelevation Development. . . . . . . . . . . . . . . . . . . . . 220

TABLE 7.7: Difference Between 2-D and 3-D $K$ Values for Crest Vertical Curves Combined with Horizontal Curve. . . . . . . . . . . . . . 222

TABLE 7.8: Effect of Speed, Horizontal Curve Radius, and Superelevation Rate on 3-D $\boldsymbol{K}$ Values for Srest Vertical Curves Combined with Horizontal Curve.

TABLE 7.9: 3-D $K$ Values for Crest Vertical Curve Combined with Horizontal Curve with $90 \mathrm{~m}$ Superelevation Runoff Leng:h (No Spiral Curve). . . . . 225

TABLE 7.10: 3-D $K$ Values for Crest Vertical Curve Combined with Horizontal Curve with $90 \mathrm{~m}$ Spiral Curve at Both Ends. . . . . . . 226 


\section{LIST OF FIGURES}

FIGURE 1.1: Framework for Highway Geometric Design. . . . . . . . . . . . 7

FIGURE 2.1: Passing Manoeuvre According to AASHTO (1994) . . . . . . . . . I8

FIGURE 2.2: Passing Manoeuvre According to Vin Valkenherg and Michacel

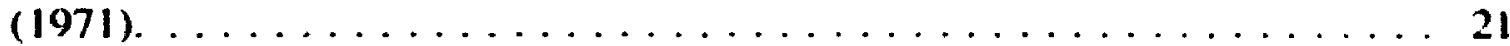

FIGURE 2.3: Time-Space Diagrams for Critical Passing Manoeuvre (Glennon.

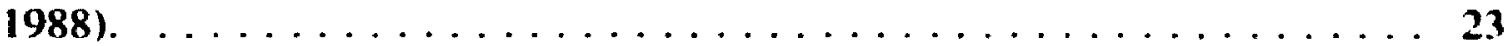

FIGURE 2.4: Forces Acting on a Passenger Car in a Bicycle Model

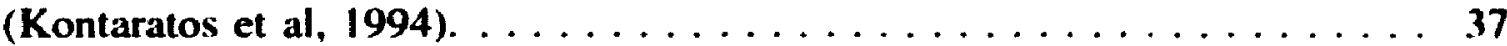

FIGURE 2.5: Vehicle Dynamics Model Axis System and Degrees of Freedom

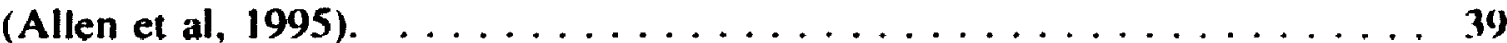

FIGURE 2.6: Coordination of Distortion Points in Horizontal and Vertical

Alignments (Smith and Lamm, 1994). . . . . . . . . . . . . . . 43

FIGURE 2.7: Effect of Superimposed Sag Vertical Curves on Appearance of

Horizontal Curves (Smith and Lamm, 1994). . . . . . . . . . . . . 48

FIGURE 3.1: Assumptions Required for $\boldsymbol{G}=\boldsymbol{m} . \ldots \ldots \ldots \ldots$

FIGURE 3.2: Actual Headway at the End of a Completed Pass. . . . . . . . . . 54

FIGURE 3.3: Time-Space Relationship for Two Vehicles with Speeds $v_{1}$ and

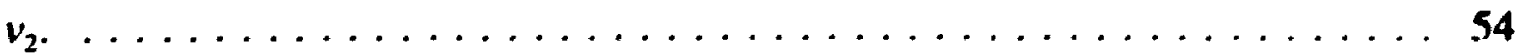

FIGURE 3.4: Time-Space Diagrams of Aborted and Completed Passes. . . . . . . 59 
FIGURE 3.5: Time-Space Diagram for the Passing Manoeuvre Between Pass

Initiation and Critical Position.

FIGURE 3.6: Relative Positions of the Passing and Impeding Vehicles up to

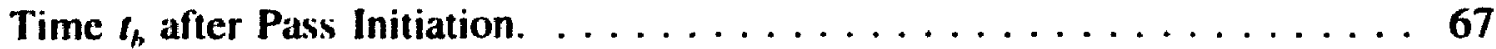

FIGURE 3.7: Time-Space Diagrams for the Simplified Model. . . . . . . . . . 71

FIGURE 3.8: Validation of the Model for Required PSD . . . . . . . . . . 81

FIGURE 3.9: Comparison of Required PSD of the Model and Current Practice. . . 82

FIGURE 3.10: Comparison of Minimum Length of Passing Zones of the Model

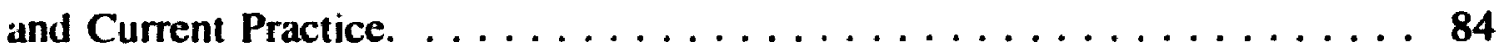

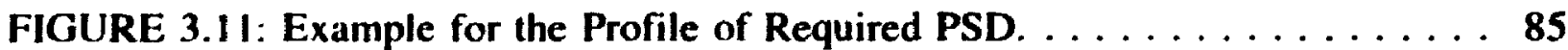

FIGURE 3.12: Determination of the Beginning and End of Passing Zones. . . . . 87

FIGURE 4.1: General Procedure: Sight Line Tangent to Circular Curve

(Continuous Obstruction) $\ldots \ldots \ldots \ldots \ldots \ldots \ldots \ldots$

FIGURE 4.2: Determination of Coordinates on Spiral Curves. . . . . . . . . . . 92

FIGURE 4.3: General Procedure: Sight Line Tangent to Spiral Curve

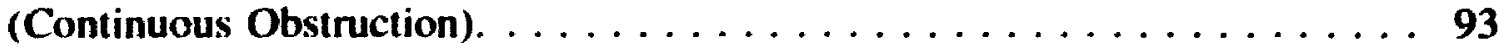

FIGURE 4.4: General Procedure: Intersecting Straight Segments Without

Curves (Continuous Obstruction) $\ldots \ldots \ldots \ldots \ldots \ldots \ldots$

FIGURE 4.5: Special Case: Simple Horizontal Curve (Continuous Obstruction). . . 99

FIGURE 4.6: Special Case: Intersecting Straight Segments Without Curves

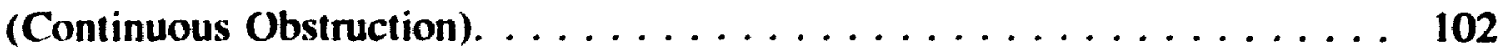

FIGURE 4.7: Sight Distance Limited by Crest Vertical Curve. . . . . . . . . 108 
FIGURE 4.8: Development of Sight-Hidden Dips. . . . . . . . . . . . I 109

FIGURE 4.9: Sight Distance Limited by Overpass on Sage Vertical Curve. . . . 111

FIGURE 4.10: Parametric Elements for 2-D Horizontal and Vertical

Alignments. $\ldots \ldots \ldots \ldots \ldots \ldots \ldots \ldots \ldots \ldots \ldots \ldots \ldots \ldots \ldots$

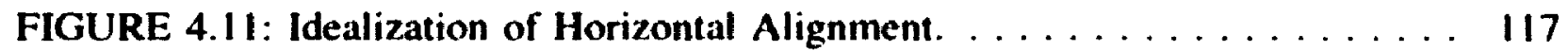

FIGURE 4.12: Idealization of Vertical Alignment. . . . . . . . . . 119

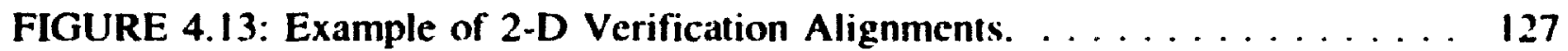

FIGURE 5.1: Rectangular Parametric Elements. $\ldots \ldots \ldots \ldots \ldots \ldots \ldots \ldots$ 1.31

FIGURE 5.2: Triangular Elements. $\ldots \ldots \ldots \ldots \ldots \ldots \ldots \ldots \ldots \ldots \ldots$

FIGURE 5.3: Idealization of Tangents, Vertical Curves, and Horizontal Curves into Parametric Elements. . . . . . . . . . . . . . . . .

FIGURE 5.4: Idealization of Combined Vertical and Horizontal Alignment. . . . 139

FIGURE 5.5: Lateral Obstructions and Their Representation by 4-Node

Elements. $\ldots \ldots \ldots \ldots \ldots \ldots \ldots \ldots \ldots \ldots \ldots \ldots \ldots \ldots \ldots \ldots$

FIGURE 5.6: Modelling of Overpasses. $\ldots \ldots \ldots \ldots \ldots \ldots \ldots \ldots \ldots \ldots$

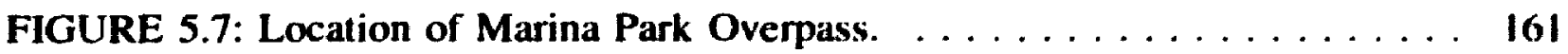

FIGURE 5.8: As-Built Horizontal and Vertical Alignments of Marina Overpass,

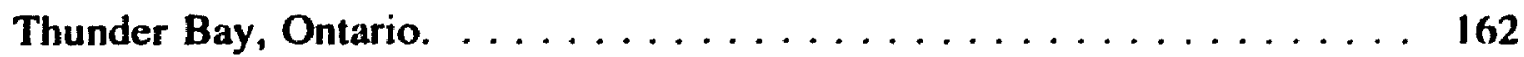

FIGURE 5.9: Original As-Built and Current Vertical Alignments After Bridge

Deck. ...................... 163

FIGURE 5.10 : Sight Distance Targets Used in Field Measurements. . . . . . 165

FIGURE 5.11: The Element Net for Modelling Marina Overpass. . . . . . . 166 


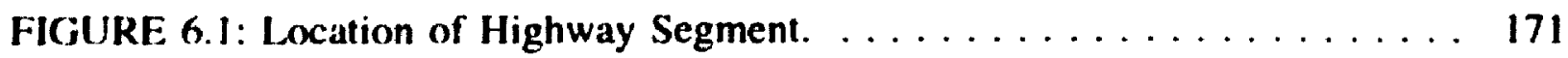

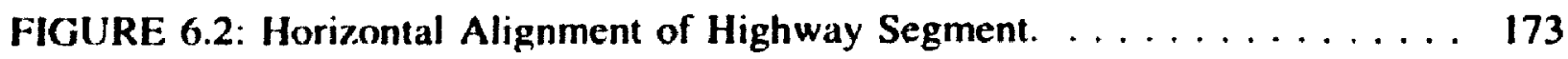

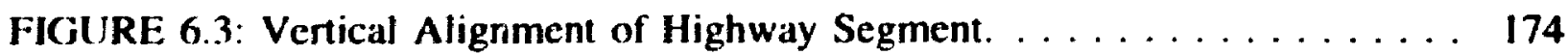

FIGURE 6.4: Examples for Different Cross-Sections of Highway Segment. . . . 175

FIGURE 6.5: Element Net for 2-D Horizontal Alignment. $\ldots \ldots \ldots \ldots \ldots$

FIGURE 6.6: Example of Element Net for 3-D Combined Alignment. . . . . . 183

FIGURE 6.7: Lateral Obstruction in 2-D Horizontal Alignment from Station

$(13+100)$ to Station $(13+150) \ldots \ldots \ldots \ldots \ldots \ldots \ldots \ldots$

FIGURE 6.8: Profile of Available PSD in 2-D and 3-D Alignments. $\ldots \ldots \ldots 188$

FIGURE 6.9: Percentage Difference Between 2-D and 3-D Sight Distances. . . . 189

FIGURE 6.10: Profile of Available PSD According to MUTCD (1995)

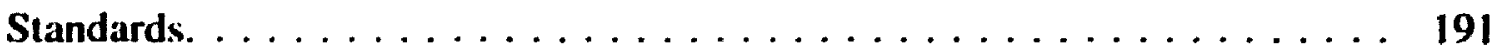

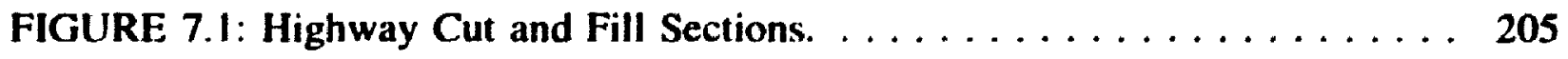

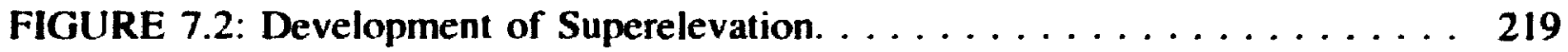

FIGURE 7.3: Combined Alignment on Cut Section on Highway 17. . . . . 229 


\section{CHAPTER 1}

\section{INTRODUCTION}

Since ancient times, advancement and prosperity of civilizations have heen very much dependent on the availability of a reliable transportation system, with roads representing a major component. Subsequently, it is not surprising to know that both the Roman and Persian Empires had built good roads which served not only as commercial tools but also as military and power instruments (Meijer and Van Nijf, 1992). Currently, in spite of the advancements in other transportation modes, highways are still holding their importance in both the economic and military fields. Realizing this fact, it was reported in a study published by the World Bank that a transport infrastructure is a prerequisite of economic development (Adler, 1987). It was also reported in the same study that it is not unusual for traffic growth to be two to three times as large as the rise in national income. Therefore, it has been the interest of the World Bank to encourage developing the transport sector in the less developed countries (Van Der Tak and Ray, 1971). Furthermore, the importance of highways increases even more in the US and Canada where, according to 1977 statistics, $5 \%$ of the world's population own $43 \%$ of the motor vehicles (Oglesbey and Hicks, 1982).

In Canada, highways have evolved from very primitive narrow trails made hy natives to connect rivers and lakes, where the canoe was the basic transportation means, to one of the most advanced road networks in the world. The service provided by this network was described as "today, no other country, in relation to its size and population. 
is better served" (Guillet, 1966) These roads served $93 \%$ of all domestic travel by Canadians in 1990 as will as the freight travei of more than 84 billion tonne-kilometres in 1988, with an expected continuous increase in these figures until the year 2000 (TAC, 1990). In the fiscal year 1988/89, the federal, provincial, and municipal expenditures on the road network, whose length totalled 879,530 2-lane kilometres, were more than $\$ 8.4$ billion. In the US in the year 1994, federal, state, and local governments spent more than $\$ 90$ billion on a total of $3,906,544$ miles of highways (BTS, 1996). This

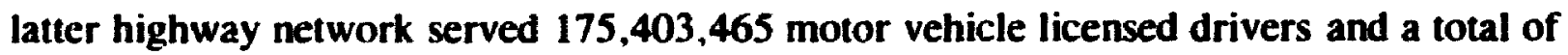
$133,929,661$ cars; $3,718,427$ motorcycles; 670,423 buses; and $63,445,280$ trucks. Outperforming all other domestic transportation modes, in 1994, American highways accounted for $2,364,384$ million vehicle-miles ( $98.5 \%$ of all domestic vehicle-milage) and $3,937,765$ million passenger-miles ( $90.7 \%$ of all domestic passenger-milage). In addition to passenger travel, by carrying 908 billion ton-miles of freight $(25.6 \%$ of all domestic ton-milage of freight), highways played a significant role in the American freight transportation.

However, one key element in designing modern highways is the increasing awareness of the possible highway and motor vehicle impacts. As a result, highway design has become a very complex process in which designers and agencies of different specialities and interests cooperate to account for traffic safety and efficiency, driver comfort, social and environmental impacts, highway aesthetics, and economic benefits. This complexity in the design process was reported by the Federal Highway Administration of the US (FHWA). It was stated that "highway transportation goals 
become broader and more difficult to achieve as time goes" (FHWA, 1972). Subsequently, a rational highway design process should involve the following stages: planning, route selection, geometric design, and structural design.

\subsection{Highway Design Process}

The planning phase is a never-ending process whose main objective is "the establishment of a highway network capable of accommodating all highway travel in an orderly, safe. efficient, and economical manner" (Michael, 1960). Due to the increasing public concern about the social and environmental impacts of highways and motor vehicle transportation, the decisions ma.le in this phase are usually influenced by public organizations and representatives. As a result, the decisions have to be acceptable not only professionally and economically but also politically. An approach for the planning phase including the decision-making process and the factors considered can be found in the literature (Khisty, 1990; Oglesbey and Hicks, 1982). If, at a certain time, a need for a highway between certain points is established, the possible alternative routes are compared to determine the most economical route for the highway to follow. Such an economic comparison must consider the costs of both construction and right-of-way and the existence of any control points (Young et al, 196n).

The highway geometric design is the phase which follows the route selection. As defined in the literature, highway geometric design is the proportioning of the visible elements of the highway (Easa, 1995; Garber and Hoel, 1988; King and Harkins, 1982; Noble, 1960; Wright and Paquette, 1979). The laws of motion (kinematics and 
dynamics), the vehicle characteristics, and the ability and psychology of the road users are incorporated in this phase to produce safe and efficient highways. In order to ensure the safety and efficiency of the designed highways, each country has established a set of design standards to suite its traffic characteristics and environmental conditions. However, as reported by Krammes and Garnham (1995), the design standards used in most countries have far more similarities than differences. The most common set of design standards is found in the design guide developed by the American Association of State Highway i 1 Transportation Officials, known as the AASHTO green book, (AASHTO, 1994). This design guide is used by the different states as a basis to develop their own standards. In Canada, the provinces use the Manual of Geometric Design Standards for Canadian Roads published by the Transportation Association of Canada (TAC, 1986), formerly known as the Roads and Transportation Association of Canada (RTAC). as their design standards or as a basis for their own. Another set of standards is used for highway marking and can be found in the Manual of Uniform Traffic Control Devices which is available in both Canadian and American editions (MUTCD, 1976; MUTCD, 1988).

Finally, the structural design of highways involves the selection of the materials used in the different pavement layers, including any required treatment for the subgrade, and thickness design for each layer. Accordingly, the designed pavement is expected to sustain the traffic loads and the environmental conditions during its design life. Detailed explanation of the principles of pavement structural design can be found in the literature (Huang. 1993: Yoder and Witczak. 1975). Also, design guides for flexible and rigid 
pavements are given by AASHTO (1986) and the Portland Ciment Assokiatten (PC $A$. 1984). Currently, extensive research work is being conducted mainly by l's and partly Ey Canada to enhance the pavement design standards. The rescarch program, known as the Strategic Highway Rewearch Program (SHRP), lanted for five years ind ended in l(w)3. Since then. FHWA. AASHTO. and the Transportation Research Board (TRIB) hate leeen working on the implementation of the new SHRP findings and products (FHWA. (M)5).

Among the different phases of the highway design process. the geometric design phase is the most related to traffic safety. This phase is the broad subject of this research where the current standards of highway geometric design are reviewed. The'n. extensums to these standards are suggested. The following vections expiain the concerms with current standards and the objectives of this reseatreh.

\subsection{Present Status of Geometric Design}

Prior to the design of the different elrments of the highway, traffic comtrohs. such an the design speed and highway classification, are selected. Then, in the geometric design phase. the designer uses the standards to design the highway alignment and the cross section, including the roadside and other special elements such as acceteration. deceleration, and truck lanes. These design standards must ensure that the resulting highways satisfy sight distance, vehicle stability, driver comfort, drainage, and highwaly aesthetics. Also, the design must not violate the practical limitalions of driver abilities. vehicle characteristics, and topography. Moreover. the design process should account for the variations in drivers' performances which result from the variations in educialion. 
experience, and age. Each of the design bases and constraints influences the design of all highway elements, as shown in Figure 1.1.

However. although geometric design standards have been revised several times during the last 40 years, the theoretical bases and fundamentals of these standards have not changed since the first design guide issued in $10^{\circ}+$ by the American Association of State Highway Officials (AASHO. 1954). For example, although the 3-D nature of highway geometric design is a well known fact, the highway alignment is still designed as separate 2-D horizontal and vertical alignments. On this point. it was reported that "the aligmment of a highway is a three-dimensional problem ... However, in highway design practice. three-dimensiomal design computations are cumbersome" (Mannering and Kilareski. 1990). Convinced of the difficulty of establishing 3-D-based standards and recognizing the need for an assessment of the combined alignment, AASHTO and TAC design guides have offered general guidelines to enhance the coordination of horizontal and vertical alignments. However, these guidelines are far less than satisfactory. As a result, Smith and Lamm (1994) concluded that "Ihree-dimensional alinement, a very complex component in the highnay geometric design process, still represents the neakest link in the overall design of highuays".

Moreover, the highway geometric design standards have been criticized by many researchers in studying certain areas. For example. Harwood and Glennon (1977) stated "in the age of interactive graphics, automatic photogrammetric plotting. freeway surveillance and control ... the highway community still designs and marks passing zones on mo-lane rural highways according to false and archaic priv '"lec". Eleven years after 


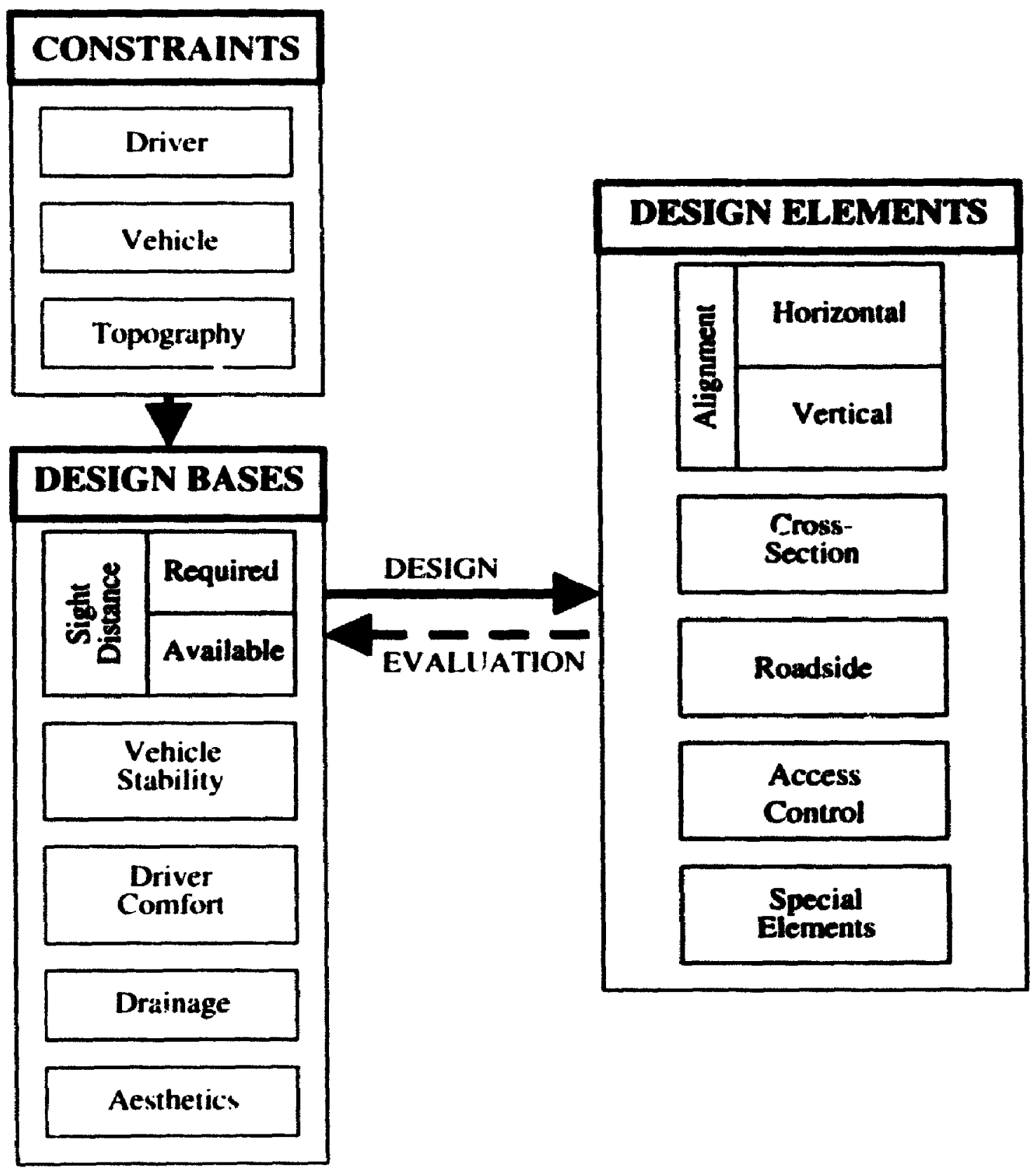

FIGURE 1.1: Framework for Highway (iermetric Design. 
this comment, It was repeated by (ilennon (1988) on the same subject of passing zones that the hegwat community still clings to false and archaic principles". The consideration of the stopping sight distance has also been criticized by Neuman (1989) who stated "hhere is continuing, growing dissatisfac tom among many design engineers nith the current policv and seneral approach w the subject". Furthermore, on the same pomt. Hall and Turner (1989) stated "enginters are finding that if is expensive fo comply. nith the current sfandards, especialls in the recomstruction of existing hightuats".

Generally, the observations of the deficiencies associated with the current sandards have teen summarized by Glennon, 1989 ) who commented on the 1984 edition of the AASHTO design guide that "efforrs were inadequate to make the Green Book

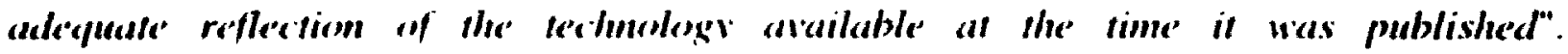
Interestingly. most of the deficiencies pointed out by Gilennon (1989) have not been addressed in the subsequint editions of the AASHTO green book, and the previous comment can still be applied to the most recent edition published in 1994.

\subsection{Problem Definition}

As stated earlier. this research focuses on the geometric design phase. The current pratice and standards of highway geometric de:ign are reviewed against the framework shown in Figure 1.1. As shown in the figure. all the destgn elements (horizontal and vertical alignments. cross-section. roadside, access control. and special elements such as acceleration lanes, climbing lanes, ...) should be designed jointly. if a new highway is being designed. Furthermore, when an existing highway is being evaluated, the effect of 
all these elements should also be considered fointly. However. as mentioned anther. ank of the main deficiencies in the current practice of highwa! geometric de-sgn is that wach element is designed separately instead of a combined aigmment af all clements in (1) analysis. As a result. complete achevement of the ohpectives of highwaly geomettic design is not guaranteed with the use of the current standards, espectally. "llice devgluet is not very experienced. On the other hand. the c(onsiderallen (1) the 3-1) highwaly alignment should dramatically reduce the risk of any design defiectency and the asweratted costs for its subsequent fixation.

In summary, the concerns in the current geometric design standards can in stalts as follows:

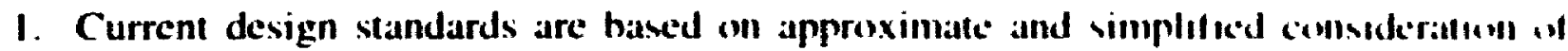
each highway clement in a 2-D projectuon and xeparately from the ofluer element

2. Moreover, in this simplified 2-D design, the current standards call deal .. ' wilh all isolated simple curve, in a horieontal or vertical alignment.

3. Although other research works have comsdered compound and reverve curves. they have failed to consider general alighments with successtve curves and shent tallfints 4. Oversimplified 2-D design standards do not guarantece a sillsfactory highwaty de vgll

5. Highway alignment design should be based on 3-D analysis.

6. The 3-D nature of highway alignnent has not been exploted in deven trecaluse af its complexity. 


\subsection{Objectives and Scope}

Consequently, the main objectives of this research are:

1. To identify and evaluate the weakness in the current geometric design prac : : where each element is designed separately in a 2-D projection.

2. To develop a design methodology to account for the 3-D nature of highway alignment.

3. To study the effect of 3-D analysis on different highway elements.

4. To quantify the ditference between 2-D and 3-D alignment on highway marking and design.

5. To revise the available models for the required passing sight distance and the minimum required length of passing zones.

It should be noted that the scope of this research is limited to the design of rural highways. Also, as indicated by the hatching in Figure 1.1, the research focuses mainly on the sight distance and its impact on the different highway elements. In addition, since stopping sight distance is currently being addressed in the National Cooperative Highway Research Program (NCHRP) Project 3-42, is has not been included in this research.

The next chapters of this thesis are organized as follows:

- Chapter 2 presents a detailed review of the consideration of 3-D highway alignments with a greater focus on the work conducted in the field of sight distance and its relation to the different design elements.

- Chapter 3 explains the revised model developed in this research to determine the required passing sight distance. Also, developed models for the length of passing 
zones and the profile of the required passing sight distance with the evolvement of the pass are presented.

- Chapter 4 explains the methodologies developed in this research to determine the available sight distance on complex 2-D horizontal and vertical alignments. Either alignment is considered separately from the other one.

- Chapter 5 explains a new methodology to determine the available sight distance on general 3-D combined horizontal and vertical alignments.

- Chapter 6 presents an application of the developed models for required and available sight distances in marking passing and no-passing zones. A practical example of a 7km segment on Highway 61 is presented.

- Chapter 7 presents a further application of the developed models for available sight distance in alignment design with a quantification for the consequences of ignoring the 3-D nature of highway geometric design.

- Chapter 8 presents the main conclusions and findings of this research and recommendations for further research work required to establish 3-D design standards. 


\section{CHAPTER 2}

\section{REVIEW OF CURRENT DESIGN PRACTICE \\ AND EXISTING RESEARCH WORK}

In this chapter, the previous research work and the current practice of highway geometric design are reviewed against the framework presented in Figure 1.1. The review focuses on the design bases (sight distance, vehicle stability, driver comfort, drainage, and aesthetics) and how they affect the different design elements. Although the design constraints influence the design elements, they are not explicitly included in this review. As indicated by the hatching in the figure, more emphasis is put on the sight distance which is the main focus of this research.

\subsection{Sight Distance}

Ideally, geometric design should ensure that any object on the pavement surface is visible to the drivers within the normal eye sight distance. However, because topographical constraints make such a design impractical, roads are designed to provide drivers with at least the minimum sight distance required for safe and efficient operation. The sight distance is defined in the AASHTO guide (1994) as the length of the highway visible to the driver. The role of the sight distance in safe and efficient traffic operation has been emphasized in both AASHTO and TAC guides.

Although highway professionals agree on the correlation between sight distance and safety, many researchers have reported that not enough studies have been carried out 
to quantify this relationship (TRB, 1987: Glennon, 1987; Olson et al, 1984). One of the main reasons for the difficulty associated with establishing such correlation is that sight distance is always associated with a horizontal and/or vertical curvature. Thus, it is difficult to separate sight distance effects from other highway elements. Moreover. the accidents resulting from deficient sight distance are event oriented. For such accidents to occur, a series of events must first take place at the critical section with deficient sight distance.

In the state-of-the art presented in Report 270 by the National Cooperative Highway Research Program (NCHRP), Olson et al (1984) summarized the findings of 20 studies on the relationship between highway elements, including sight distance, and safety. Among these studies, a weak relationship between sight distance and safety was reported in only one study while the remaining 19 concluded that safety increases as the alignment and sight distance improve. However, because all of these studies included the effect of the alignment, combined with sight distance, another study was conducted to neutralize this parameter (Olson et al, i984). In this study, the accident counts on 10 matching pairs of vertical curves were compared. Each pair was selected so that the two curves had identical traffic and alignment features. One of the two curves had a limited sight distance while the other one had a sight distance more than the minimum required sight distance according to the 1965-AASHTO guide. The results showed that the accident counts on the control sections were significantly lower than the accident counts on the sections with limited sight distance. 
Therefore, design standards state that designers must provide, at every point along the highway, a sight distance sufficient for a below-avcrage driver or vehicle to stop before hitting an unexpected object on the pavement (AASHTO, 1994; TAC, 1986). Such a distance is known as the stopping sight distance (SSD). Moreover, a longer sight distance has been recommended in some situations where the driver has to make a complex decision. Such a distance is known as the decision sight distance (DSD). Design values for DSD are given in the AASHTO and TAC guides. However, DSD is extremely variable and must be analyzed on a single-situation-basis.

As for efficiency, "if operational efficiency is to be built into the road, for higher Iraffic volumes, then lengths of road with sufficient sight distance may have to be provided for drivers to overtake slower vehicles safely" (Kosasih et al, 1987). Clearly, since overtaking slower vehicles on multi-lane highways can be performed with a lane change without travelling in the lanes of the opposing traffic, this requirement is applicable for two-lane highways only. The role of the availability of sufficient passing sight distance (PSD) on the level-of-service of two-lane highways has been emphasized In the Highway Capacity Manual (HCM, 1994). As shown in the HCM, the volume-tocapacity ratio of two-lane rural highways may be reduced by more than $30 \%$ if the percentage of the length of no-passing zones increases from zero to $100 \%$. Moreover, long stretches of two-lane rural highways with no opportunity for passing slower vehicles could cause drivers to carry out erratic and hazardous passing manoeuvres (Persaud, 1992). For example, in 1987, while the head-on non-intersection collisions on two-lane highways in Ontario, where erratic passing is the most probable cause, represented just 
$2 \%$ of all accidents, they accounted for $17 \%$ of the fattal accidents. Therefore, AAsuro and TAC guides have recommended providing sufficient PSD on two-lane rural highways at frequent intervals and for substantial portions of the highway length. The collisions due to erratic passing can also be reduced by a good marking of passing and no-passing zones to assist drivers, especially the less experienced, decide whether to pass or trail a slower vehicle. Such a marking is based mainly on sight distance requirements.

Realizing the importance of sight distance in highway geometric design. considerable research work has been directed to answer two vital questions: how long should the required sight distance be?, and how long is the actually available sight distance? In the following sections, the current models to determine the required sight distance are reviewed and discussed. Also, the existing models for the available sight distance, with the incorporation of these models in the highway design and evaluation, are presented.

\section{I.I Required Stopping Sight Distance (SSD)}

As stated earlier, SSD is a major element in the safe operation on any highway. In the historical review of the development of the required SSD. Hall and Turner (1989) showed that the importance of SSD has been recognized as early as in 1914 (Agg. 1916; Blanchard and Drowne, 1914). In 1940, the AASIIO policy on sight distance presented an analytical model for calculating the required SSD based on the laws of dynamics (AASHO, 1940). In this model, which is still in use until today, the SSD is measured from the driver eye to the highest point of a small stationary object on the road. The 
required SSD in the model is the sum of the distance travelled during the perceptionreaction time and the braking distance, B.D., which can be calculated as follows:

$$
\text { B.D. }=\frac{V^{2}}{254(f+G)}
$$

where

$$
\begin{aligned}
& V=\text { initial speed of the vehicle }(\mathrm{km} / \mathrm{h}) . \\
& f=\text { coefficient of friction between the tires and the roadway, and } \\
& G=\text { highway grade in decimal fraction (positive if upward and vice versa). }
\end{aligned}
$$

Although this model has not changed since then, many studies have been conducted to revise the values of its parameters. Among them is a comprehensive study conducted by Olson et al (1984). The study included the quantification of the perceptionreaction time, height of the driver eye, height of the object, and the coefficient of friction. Furthermore, a new concept of deceleration with steering control for a passenger car and a truck was presented to ensure safety and comfort on horizontal curves. Also, a new and rational approach to determine the required SSD based on highway functional classification was suggested by Neuman (1989). However, the most recent edition of the AASHTO guide in 1994 failed to incorporate this approach in the design standards. A more recent comprehensive revision for the model is being conducted in the NCHRP Project 3-42 (Fambro et al, 1995). 


\subsubsection{Required Decision Sight Distance (DSD)}

In some complex situation, the perception of information may he difficult and/or the appropriate action may be unexpected or unusual. In such situations, SSD is usually not enough to ensure traffic safety. As a result. the AASHTO guide recommends the provision of a longer sight distance known as the decision sight distance (DSD). The DSD has been defined in both AASHTO and TAC guides as "the distunce required for a driver to detect an unexpected or otherwise difficult-ro-perceive information somarce or hazard in a roadway environment that may be visually cluttered, recognise the hasard on its threat potential, select an appropriate speed and path. and initiate and complete the required safety manoeuvre safely and efficiently". A similar sight distince, called manoeuvre sight distance, is also recommended in the Australian standards presented by the National Association of Australian State Road Authorities (NAASRA, 1980). Although design values of the DSD are given in each of these design guides. DSD should be determined on a single-situation-basis. This is mainly due to the fact that it depends on the complexity of the situation and the volume of traffic.

\subsubsection{Required Passing Sight Distance (PSD)}

(i) AASHTO Model. Based on the results of field studies conducted in and before 1958 and as shown in Figure 2.1, AASHTO presented a model to calculate the PSI). S. ats follows:

$$
S=d_{1}+d_{2}+d_{3}+d_{4}
$$




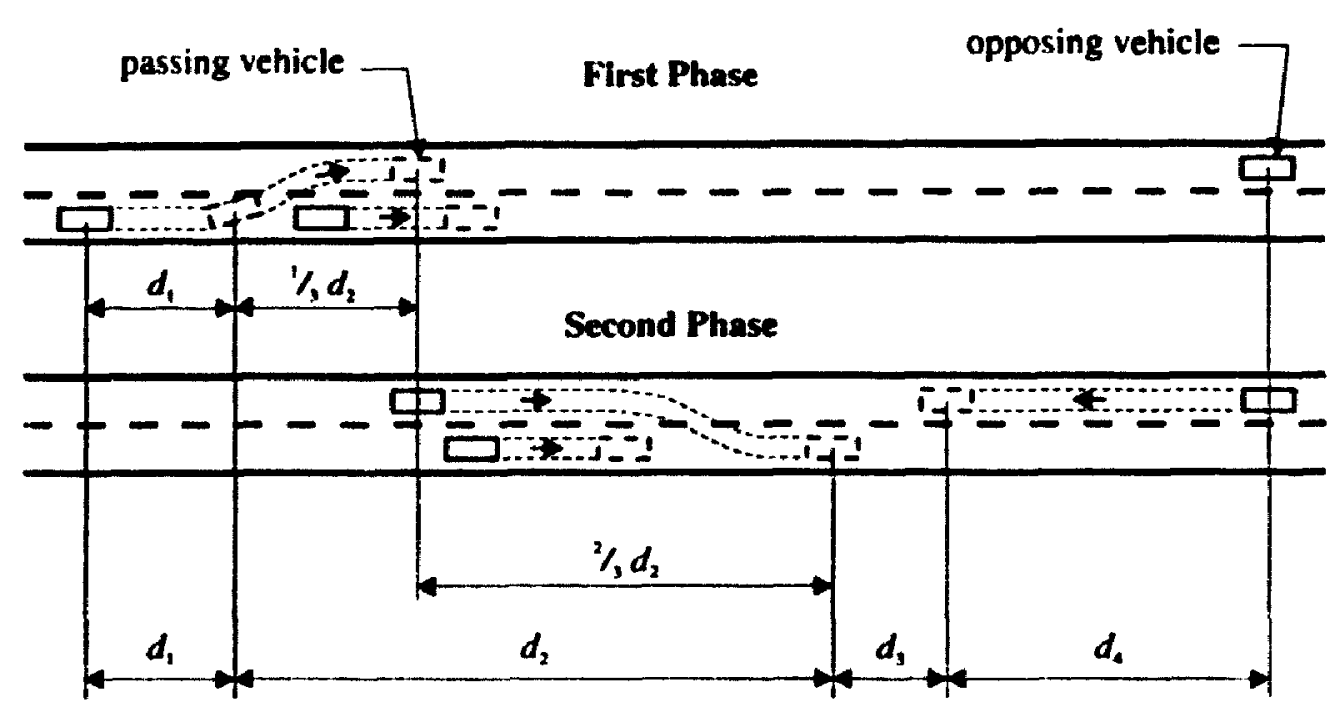

FIGURE 2.1 : Passing Manoeuvre According to AASHTO (1994).

$d_{2}=$ distance travelled by the passing vehicle while occupying the left lane (time elapsed $\left.=t_{2}\right)$,

$d_{3}=$ clearance distance between the passing and opposing vehicles at the end of the pass, and

$d_{4}=$ distance travelled by the opposing vehicle for two-thirds of the time the passing vehicle occupies the left lane $=2 / 3 d_{2}$ (time elapsed $=t_{4}=2 / 3 t_{2}$ ).

Design values for these distances and PSD are given in the AASHTO and TAC design guides.

However, this model is not free of self-discrepancies. By taking $t_{4}=2 / 3 t_{2}$ instead of $t_{1}+t_{2}$, the model accounts for the driver's ability to abort the pass if any opposing vehicle is seen ahead during the time $t_{1}+1 / 3 t_{2}$. At the mean time, by considering $d_{1}$ and all $d_{2}$ as parts of the PSD, the model assumes that the passing vehicle is committed to complete 
However, this model is not free of self-discrepancies. By taking $t_{4}=2 / 3 t_{2}$ inste:ul of $t_{1}+t_{2}$, the model accounts for the driver's ability to abort the pass if any upposing vehicle is seen ahead during the time $t_{1}+1 / 3 t_{2}$. At the mean time. by considering $d_{1}$ and all $d_{2}$ as parts of the PSD. the model assumes that the passing vehicle is committed to complete the pass once it is initiated. Three additional flaws in the model and its design values were pinpointed by Harwood and Glennon (1977) as follows:

1. The model is incapable of determining the minimum length of the passing ronc.

2. The model uses assumed average speeds rather than the design spects, and subsequently it represents an average pass not a critical pass.

3. For high speeds, speed differentials between passing and passed vehicless were taken constant instead of being determined from field measurements.

In conclusion, Weaver and Glennon (1971) and Harwoxd and (ilennon (1977) suggested that $2 / 3 d_{2}+d_{3}+d_{4}$ would represent a more logical model for PSI). Ifowever, such a conclusion was very subjective and does not consider the other flaws in the model they criticized, and they themselves participated in developing another new mudel (cilennon. 1988: Harwood and Glennon, 1989).

(ii) MUTCD Design Values of PSD. Another discrepancy arises when comparing the PSD values given in the Canadian and American versions of MUTCI). which are used in pavement marking, and those given in design guides (AASHTO, 1994; TA(:, 1986). A comparison between these values, as shown in Table 2.1, shows that the values used in pavement marking are much shorte, than those presented in the design guides. 
TABI.E 2.1: Passing Sight Distance Requirements in Design and Marking Standards in Canada.

\begin{tabular}{ccc}
\hline \multirow{2}{*}{$\begin{array}{c}\text { Speed } \\
(\mathrm{km} / \mathrm{h})\end{array}$} & \multicolumn{2}{c}{ Required PSD (m) } \\
\cline { 2 - 3 } & $\begin{array}{c}\text { Design Standards } \\
(\text { TAC. 1986) }\end{array}$ & $\begin{array}{c}\text { Marking Standards } \\
\text { (MUTCD. 1976) }\end{array}$ \\
\hline 50 & 340 & 160 \\
60 & 420 & 200 \\
70 & 480 & 240 \\
80 & 560 & 275 \\
90 & 620 & 330 \\
100 & 680 & 400 \\
110 & 740 & 475 \\
120 & 800 & 565 \\
130 & 860 & $\ldots$ \\
\hline
\end{tabular}

Interestingly, it was reponted by Harwood and Glennon $(1977 ; 1989)$ that the reasons for selecting these minimum sight distances in the MUTCD are not stated, nor is the source given. However, they noted that these values are identical to those presented in the 1940AASHO Guide. Since these values represent a subjective compromise between PSD for delayed and tlying passes, Harwood and Glennon (1977: 1989) concluded that these values do not represent any particular passing situation.

(iii) Models Bused on the Concept of Critical Position (Point of No Return). A new concept in modelling PSD was presented by Van Valkenberg and Michael (1971). As 
shown in Figure 2.2, they considered that the distance travelled by the passing velicle can be divided into two distances: $S_{0}$ the distance during which the vehicle calt apply the brakes and pull back into the proper lane, and $S_{1}$, the distance required to complete the pass. The point beyond which the pass must be completed was called the proint if no, refum, and based on personal judgement it was assumed to cecur when the rear bumper of the passed vehicle is abreast of the middle of the passing vehicle. Then, the PSI) was taken as the summation of $S_{1}$ and $S_{2}$ plus a clearance distance, where $S_{2}$ is the distames travelted by the opposing vehicle during the time required for the passing vehicle to travel the distance $S_{1}$. Although, Van Valkenberg and Michacl (1971) presented design values for PSD based on field measurements. they did not present mathematical modelling: for their work and subsequently, these measurements can be useful for highways will specds and conditions within the range used in the field measurements only.

The same concept was used by Lieberman (1982) who called the point of no return the critical position. He defined the critical position as the point where "fhe

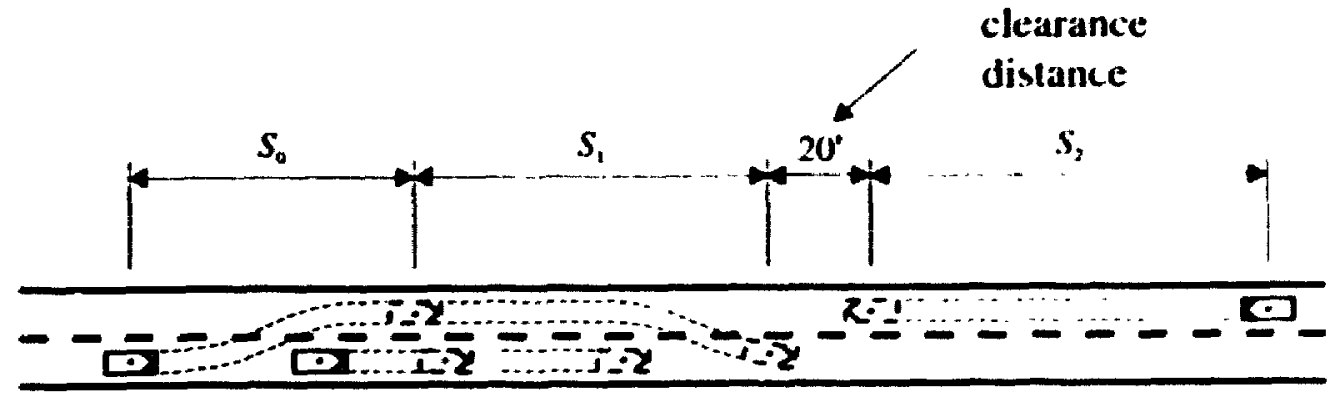

FIGURE 2.2 : Passing Manoeuvre According to Van Valkenberg and Michael (1971). 
decision by the passing vehicle to complete the pass will afford it the same clearance relative to the ancomming vehicle as will the decision wo abort the pass" (Lieberman.

1982). He incorporated this definition into a mathematical model to calculate the PSD hut he assumed that the driver is committed to complete the pass, and therefore, he concluded that the AASHTO criteria for PSD were inadequate. In addition to this very conservative approach, other flaws in the modelling were identified by Glennon (1988) such as ignoring the direct effects of vehicle length and the perception-reaction time in atorted passes. Another altempt for modelling the PSD using the concept of the critical position was made by Saito (1984). However. as stated by Glennon (1988). Saito considered only the needs to abort the manoeuvre and ignored the trade-offs between the completed and aborted manoeuvres.

In 1988. Glennon (!988) presented the most comprehensive and closest modelling to the actual mechanism of the passing manceuvre. He interpreted the definition of the critical position. or the point of no return. by having a minimum acceptable headway between the nearest points of each two vehicles involved in the manoeuvre at the end of either a completed or aborted pass (for example, the rear bumper of the passing vehicle and the front bumper of the impeding vehicle in a completed pass). Then. he developed a model based on the hypothesis that at the beginning of the pass, the sight distance required to abon the pass is much less than that required to complete it and vice versa by the end of the pass. In between. there is a point, the critical position, where the sight distance required to complete the pass is equal to that required to abort it. He called this sight distance the critical sight distance. $S_{4}$. 
As shown in Figure 2.3. Glennon identified the time-space diagram for completed and aborted passes from the critical position. Equating the distances between the front bumper of the passing and impeding vehicles at the critical position. $\Delta_{r}$ and $\Delta_{r}^{*}$, and the critical sight distance, $S_{c}$ and $S_{c}$. and assuming a one-second minimum acieptable headway, the model was formulated as follows:

$$
\begin{aligned}
& \Delta_{c}=L_{p}+m\left[\frac{2 m+L_{1}+L_{p}}{2 v-m}-\sqrt{\left.\frac{4 v\left(2 m+L_{1}+L_{p}\right)}{d(2 v-m)}\right]}\right. \\
& S_{c}=2 v\left[2+\frac{L_{p}-\Delta_{c}}{m}\right]=2 v+\frac{2 v\left(L_{p}+m-\Delta_{t}\right)}{m}
\end{aligned}
$$

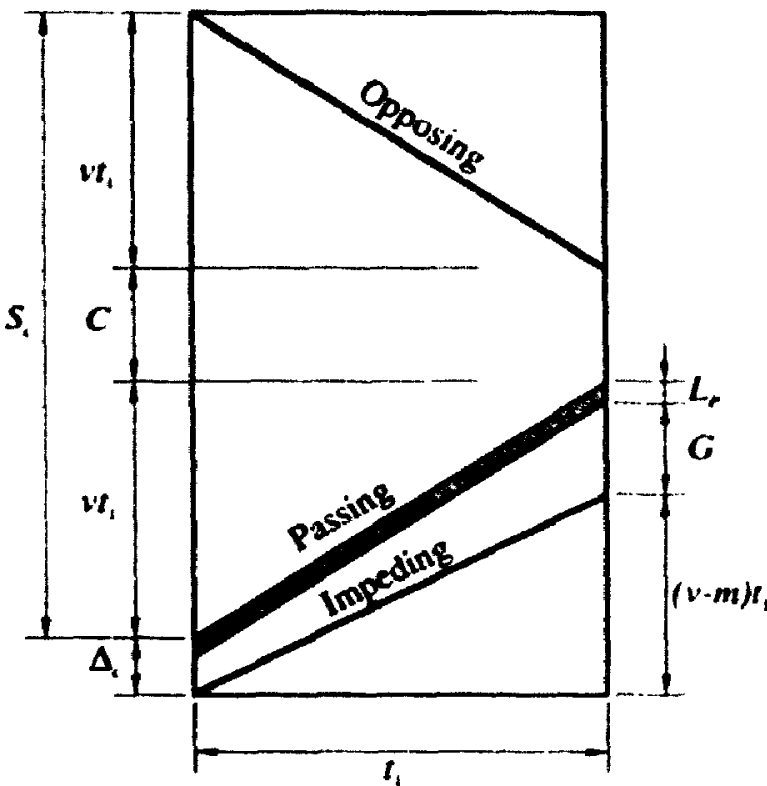

Completed Pass

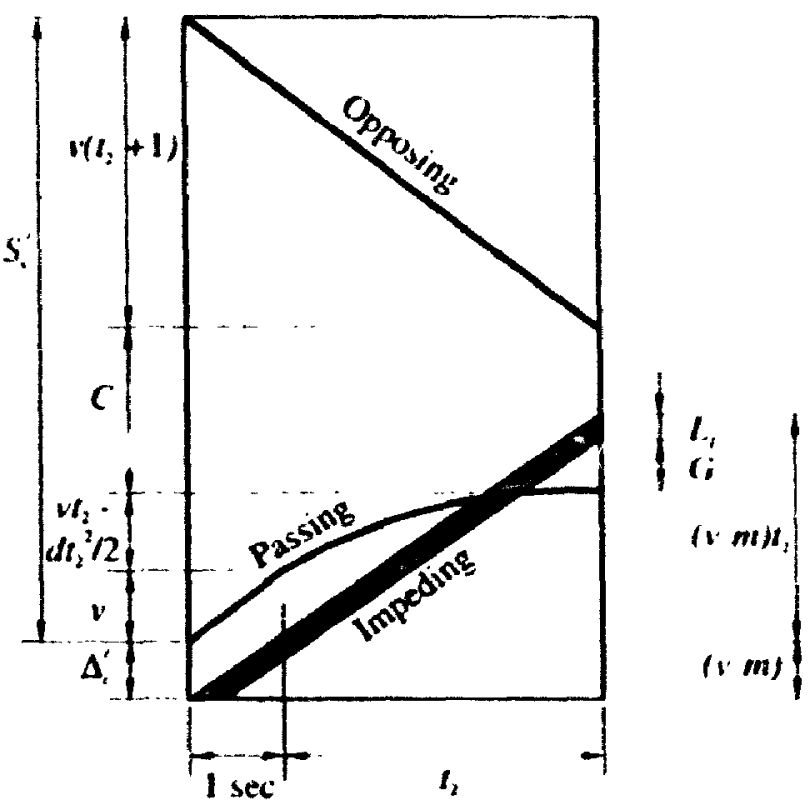

Abonted Pass

FIGURE 2.3: Time-Space Diagrams for Critical Passing Manoeuvre (Glennon, 1988). 
where

$$
\begin{aligned}
& v=\text { design speed, } \\
& m=\text { differential speed between passing and impeding vehicles, } \\
& L_{p}=\text { length of passing vehicle, } \\
& L_{\boldsymbol{l}}=\text { length of impeding vehicle, and } \\
& d=\text { deceleration rate. }
\end{aligned}
$$

Some of Glennon's assumptions were revised by Rillet et al (1990) and a modified model was developed. The main points addressed in this modified model are:

1. The value of $G$ in either a completed or aborted pass was related to the speed $(v-m)$ rather than the differential speed $m$ used in Glennon's model.

2. In aborting the pass, the passing vehicle was assumed to decelerate to a minimum terminal speed, $v_{m m}$, and then, maintain this speed until it is back in the right lane.

3. When reaching the critical position, the passing vehicle may have not completed the acceleration to reach the speed $v$ yet.

The consideration of these assumptions resulted in PSD requirements much longer than those resulting from Glennon's model. Interestingly, due to the great complexity and conservatism associated with Rillet's model, some researchers have preferred using Glennon's model which is much simpler (Good et al, 1991; Sparks et al, 1993). However, a clwer inspection of both models would establish a need for revising the assumptions involved in each of them. A detailed examination of these assumptions is given in the following chapter. 


\subsubsection{Minimum Length of Passing Zones}

In order to ensure the safety of the passing manoeurres, the American MUTCD states that the length of the passing zone must not be less than $122 \mathrm{~m}$ (100) $\mathrm{m}$ in the Canadian MUTCD). Also, as shown in Table 2.2, different lengths, depending on the speed limit. are used in Ontario (MUTCD, 1995). However, the hasis for these lengths is not documented in any of the MUTCD editions.

TABLE 2.2: Minimum Length of Passing Zones Used in Ontario (MUTCD, 1995).

\begin{tabular}{cc}
\hline $\begin{array}{c}\text { Operating Speed } \\
(\mathbf{k m} / \mathbf{h})\end{array}$ & $\begin{array}{c}\text { Minimum Length of a } \\
\text { Passing Zone }(\mathbf{m})\end{array}$ \\
\hline 50 & 120 \\
60 & 160 \\
70 & 200 \\
80 & 240 \\
90 & 280 \\
100 & 320 \\
110 & 360 \\
\hline
\end{tabular}

In the study conducted by Van Valkenberg and Michael (1971), the required minimum length of passing zones was suggested as $1 / 3$ of the distance travelled by the passing vehicle, i.e., $1 / 2$ the distance $S_{0}$ in Figure 2.2. Subsequently, lengths shorter than the $122 \mathrm{~m}$ stated in the MUTCD were suggested for speeds up to $96 \mathrm{~km} / \mathrm{h}$. However, no experimental or analytical evidence was presented to support this subjective 
assumption. Moreover, these suggested short lengths were in disagreement with the findings of many studies as summarized by Harwood and Glennon (1977). For example, Jones (1970) studied the safety and efficiency of three short passing zones of 122,195 , and $268 \mathrm{~m}$, with similar traffic and geometric features. In addition, the performance on these shor zones was compared to the performance on two longer zones of 500 and 792 m length. With a speed limit of $113 \mathrm{~km} / \mathrm{h}$ on all sites, fewer than $9 \%$ of the passing opportunities on the short zones were accepted by the drivers compared to 22.8 and $41.0 \%$ on the 500 and $792-\mathrm{m}$ zones, respectively. Mol eover, based on subjective rating, the 122-m zone experienced forced to violent returns in $63 \%$ of the passes compared to 45 and $10 \%$ on the 195 and $268-m$ zones, respectively. In conclusion, the short passing zones add very little to the quality of service of the highway and the passes performed on them are more hazardous than longer passing zones.

In another experimental study conducted by Weaver and Glennon (1971), the minimum length of passing zones was assumed as the suin of the distances travelled by 'he passing vehicle during the perception-reaction time and during occupying the left lane. The resulting suggested lengths were much higher than the values recommended in the MUTCD. However, the assumption is very conservative since, according to the concept of critical position, the passing vehicle can complete the pass safely once it passed the critical position. Therefore, a rational modelling of the minimum length of passing zones is unavailable. 


\subsubsection{Available Sight Distance}

As mentioned earlier, due to topographical and economical considerations. the sight distance on some sections of the highway may be restricted to a certain length. On horizontal curves, the driver's sight line may be obstructed by lateral obstructions such as trees, buildings, and cut slopes. On crest vertical curves, the sight line may be obstructed by the vertical curve itself. Also, sight distance on sag vertical curves may be limited to the farthest point covered by the vehicle headlight. Furthermore, overpasses represent sight obstructions for the underneath traffic. Therefore, designers have to check the available sight distance against the required SSD on any highway and the required PSD on two-lane rural highways.

(i) 2-D Horizontal Alignments. For horizontal curves, many models have heen developed to relate the available sight distance to the lateral clearance. Among these models is the one presented in the AASHTO guide for the case of $S \leq L$, where $S$ is the sight distance on the curve and $L$ is the curve length. The model, which can be used in case of continuous or single obstruction, is given as follows:

$$
m=R\left[1-\cos \left(\frac{90 S}{\pi R}\right)\right]
$$

where

$$
\begin{aligned}
& R=\text { curve radius, and } \\
& m=\text { lateral clearance from the obstruction to the centerline of the inside lane. }
\end{aligned}
$$


This formula is the only arialytical model presented by the AASHTO guide to relate the sight distance to horizontal alignment. Although the formula is easy and direct, it is "of limited practical value except on long curves" (AASHTO, 1990; 1994). Therefore, the AASHTO green book recommends that the designer must use graphical methods to check sight distance on horizontal curves. Furthermore, Neuman and Glennon (1984) and Glennon (1987) showed that the lateral clearance calculated by the AASHTO formula is needed only from the point at SSD/2 after the point of curve (PC) to the point at SSD/2 before the point of tangent (PT).

The other case, where the sight distance is greater than the curve length, has been studied by man; researchers. Olson et al (1984), Waissi and Cleveland (1987), Berg et al (1989), and Easi (1991a) have developed different methods to check the required lateral clearance on simple horizontal curves. Easa $(1993 ; 1994 a)$ has also studied the case of a single lateral obstruction on compound and reverse curves and developed other formulas to relate the latital clearance to the available sight distance.

Nevertheless, none of these models has considered the case of continuous obstructions. For example, according to the standards of MUTCD (1995), which is used by the Ministry of Transportation of Ontario (MTO) for establishing the no-passing zones, the available sight distance for the drivers in the inside lane is limited by a continuous obstruction represented by a theoretical shoulder of $\mathbf{3} \mathrm{m}$-width. Continuous obstructions may also be encountered due to cut slopes or trees and bushes. Moreover, none of the procedures mentioned above has been incorporated in the design standards by AASHTO or TAC. 
(ii) 2-D Vertical Alignments. As for the case of vertical alignment, the AASHTO (1994) formulas can relate the available sight distance on a simple crest or sag curve with long tangents to the curve parameters as follows:

for a crest curve:

$$
\begin{array}{ll}
L=\frac{A S^{2}}{100\left(\sqrt{2 h_{1}}+\sqrt{2 h_{2}}\right)^{2}} & S<L \\
L=2 S-\frac{200\left(\sqrt{h_{1}}+\sqrt{h_{2}}\right)^{2}}{A} & S>L
\end{array}
$$

for a sag curve:

$$
\begin{array}{ll}
L=\frac{A S^{2}}{120+3.5 S} & S<L \\
L=2 S-\frac{120+3.5 S}{A} & S>L
\end{array}
$$

where

$$
\begin{aligned}
& L=\text { length of vertical curve, } \\
& S=\text { sight distance, } \\
& A=\text { algebraic difference in grades, percent, } \\
& h_{1}=\text { height of driver eye, and } \\
& h_{2}=\text { height of object. }
\end{aligned}
$$

These formulas are to be used in designing vertical curves to satisfy SSD, and can aiso be used to evaluate the PSD requirements on two-lane highways. However, using 
these formulas to evaluate the available sight distance would produce only the minimum sight distance rather than the sight distance profile. Therefore, only the conclusion of whether a sight distance deficiency exists or not can be reached. Yet, the exact portion of the highway with deficient sight distance cannot be determined. Moreover, the previous formulas can be used only in the case of a simple vertical curve with long tangents.

For the case of unsymmetrical (compound) crest or sag curves and reverse vertical curves, the available sight distance can be determined using the models developed by Easa $(1991 b ; 1991 c ; 1994 a)$. Also, sight-hidden cips, which may develop if a crest curve is followed by a sag curve, has been modelled by Easa (1994b). Using this latter model, the designer can check the existence of a sight-hidden dip and determine the portion of the highway experiencing this sight-hidden dip. Moreover, an account of sight obstructions resulting from non-centred overpasses has been modeled (Easa, 1992). Yet, all the available models for sight distance on vertical alignments are valid only for the daytime sight distance, and no work has been conducted regarding the nighttime (headlight) sight distance on complex alignments.

(iii) 3-D Combined Alignments. Although computer programs for 3-D highway visualization have been available for a long time, none has been directed to the 3-D analysis of sight distance. With the recent advancements of computers, more software packages have been developed and have been available to highway professionals (Tanton et al. 1986). Nevertheless, as stated by Jull and Murray (1984), "as with all computer 
programs, ITEDS (a computer softwate for highway design) does not provide any new' revelations in the theory of highway design".

In a recent study. Sanchez (1994) studied the interaction between the sight distance and the 3-D combined alignment of interchange connectors. The methodology used in this study can be summarized in three main steps. First, the alignment was idealized into a net of triangular planes using a software called InRoads. Second, a perspective view of the idealized net was created by the computer. and the sight line was drawn from the driver eye to the object. Then, from the different views generated by the computer (top, side, and driver's perspective), the operator was able to determine the obstruction impeding the sight line. Also, the available sight distance was calculated using a spreadsheet. Although this methodology was successful in achieving the objectives of the study, it is clear that it is very time consuming because: (1) a large number of small planar triangular elements is required to model horizontal and/or vertical curvatures and (2) the available sight distance is determined graphically (not analytically). Therefore, such a methodology cannot be used to establish 3-D geometric design standards. As a result, the need for accurate analysis of the sight distance in 3-D alignments has been pointed out by Easa (1994a).

\subsection{Vehicle Stability and Driver Comfort}

It is essential for the highway designers to account for the stability of the vehicle on the road to avoid situations which impose excessive forces on the vehicle and may lead to single or multiple vehicle collisions. For example, the centrifugal forces imposed on a 
vehicle negotiating a horizontal curve may cause a single vehicle collision if the vehicle skids laterally or rolls over. Also, a multiple vehicle collision can result if the vehicle moves to another lane due to lateral skidding or loss of control. Similarly, a centrifugal force experienced on vertical curves must be accounted for in the design of vertical curves. In addition to the vehicle stability, the forces should not be high enough to cause a driver discomfort even if the vehicle can maintain its stability. The need to satisfy the two conditions of vehicle stability and driver comfort is one of the basic requirements in the design of horizontal alignments, vertical alignments, and cross-sections.

In the design of horizontal alignments. AASHTO has presented a simple formula to determine the minimum radius of horizontal curves that would satisfy driver comfort and vehicle stability as follows:

$$
R=\frac{V^{2}}{127\left(f_{s}+e\right)}
$$

where

$$
\begin{aligned}
& V=\text { design speed. } \mathrm{km} / \mathrm{h}, \\
& f_{s}=\text { coefficient of side friction, and } \\
& e=\text { superelevation rate. }
\end{aligned}
$$

The values of $f$, were determined based on the driver comfort criterion using a ball-bank indicator (AASHrO, 1994). It is assumed that drivers who travel at speeds higher than the design speed will experience some level of discomfort, and therefore will reduce their speeds before any significant risk of lateral skidding. Thus, the resulting radii are expected to be higher than the limiting values beyond which the vehicle stability 
is questioned. However, Harwood and Mason (1994) have noticed that the values of $f_{\mathrm{s}}$ were developed 50 years ago, and since then, vehicle design has changed significantly. Therefore, they pointed out the need to reevaluate the levels of driver comfort used by AASHTO.

In respect to vertical alignments, a significant difference can be noted between the centrifugal forces on crest and sag vertical curves. On crest curves, the centrifugal force is acting upward in an opposite direction to the vehicle's weight. Therefore. the developed forces are always too small to cause driver discomfort or vehicle instability within the limits of normal highway speeds and conditions. On sag curves, however, the centrifugal force is acting downward in the same direction of the vehicle's weight. Although there is no risk of vehicle instability, the combination of the centrifugal force and the vehicle's weight may cause driver discomfort. Therefore, AASHTO recommends an absolute minimum length of sag curves that will limit the centrifugal acceleration to less than $0.3 \mathrm{~m} / \mathrm{sec}^{2}$ using the following formula:

$$
L=\frac{A V^{2}}{395}
$$

It should be noted, however, that the resulting length is around $50 \%$ of the length required to satisfy the headlight sight distancc. Subsequently, the previous formula does not control the design unless the highway is well lighted.

Finally, the vehicle dynamics is an important consideration in the cross-section design. The pavement cross-slope on horizontal curves (superelevation rate) is selected mainly to help overcome a portion of the centrifugal force experienced on the curve, and 
thus enhance the vehicle stability and driver comfort. However, a maximum limit for the superelevation rate is set to avoid having slow and stationary vehicles skidding into the inside of the curve, especially in icy conditions. Such a maximum limit is set by each col:try according to the prevailing weather conditions (Krammes and Garnham, 1995).

The weakness of this current design practice has been addressed by several rescurchers. An important shortcoming is that the effect of the grades or vertical curves combined with the horizontal curve is completely overlooked. In addition, the main formula for horizontal curve design has a number of rough approximations which make it far from accurate. First, the vehicle is arproximated into a point mass rather than a body. As a result, the variation in the distribution of the friction forces between the rear and front tires and between the inner and outer tires is totally ignored. Second, as mentioned by Harwood and Mason (1994), the AASHTO formula assumes that drivers follow a path of constant radius equal to the curve radius. However, based on field studies, it was found that drivers tend to jversteer at some point of the curve, and sub: quently follow a more critical path (Glennon and Weaver, 197\%). Third, the formula considers only one case of driving, namely, driving with a constant speed. Therefore, it does not consider the reduction in the side friction supply in case the vehicle's brakes are applied and some longitudinal friction is used. TAC, however, pays attention to the interaction between longitudinal and side frictions by recommending increasing the stopping sight distance on horizontal curves. More detailed consideration of this interaction is found in the German design standards (Lamm, 1984). Finally. AASHTO design guide does not consider the risk of rollover due to excessive centrifugal forces. 
Although this may be justified for passenger ears which have small heights. it may not be the case for trucks. Because of their high centre of gravity. trucks usually have a higher potential for rollover (Harwood and Mason. 19(4).

In a research to evaluate the adequacy of the AASHTO standards. Harwiknd and Mason (1994) defined the margin of safety against skidding as the difference between the available tire friction and the friction demand on the curve. In order 10 avoid the weaknesses in the AASHTO formula, and based on ficld data. the friction demand for trucks was taken $10 \%$ higher than that for passenger cars 10 account for the tire-tu-tire friction variation. Also, the friction generated by Irucks was taken as $70 \%$ of that generated by passenger cars. The margin of safety against rollover was defincd as the additional lateral acceleration the vehicle can undergo before rolling which was ascunked to occur at a lateral acceleration of $1.2 \mathrm{~g}$ for passenger cars and $0.2710(0.40 \mathrm{~g}$ for irucks. In conclusion, passenger cars were found to have adequate margins of salety against skidding and rollover. On the other hand, it was found that trucks with high centre of gravity would roll over before they would skid off a dry pavement. However, the margins of safety for trucks against skidding and rollover were still adequale if they ds, not exceed the curve design speed. Although Harwoxd and Mason ( $1(x) 4)$ concluded that the AASHTO standards do not need revisions for safety concerns, it should he noted that the interaction of the vertical and horizontal alignments was not included nor was the reduction in the side friction supply when the vehicle's brakes are applied. Als,, an accurate consideration of the variation of the friction forces from tire to tire was not included. 
The interaction between downgrades and upgrades and horizontal curves was a part in a study aiming at developing guidelines for highway geometry and pavement surface characteristics to ensure adequate vehicle control during different manoeuvres. Dunlap et al (1978) studied the accident data on two turnpikes in Ohio and Pennsylvania and found ihat neither the horizontal curvature nor the combining vertical grade has an effect on the accident rate on horizontal curves. The abilitics of three different types of passenger cars to perform three different manozuvres (comering under traction. cornering and lane change. and comering and lane change plus braking) were studied using computer simulation. The highway grade was found to have a very little influence while the curvature influenced the comering under traction only. Although the AASHTO point mass formula was found to be adequate for vehicles driving at a constant speed (comering under traction). it was far from conservative if an emergency manocuvre would be performed on the curve.

Although Dunlap et al (1978) provided field and analytical evidence on the insignificance of the effect of grades combined with horizontal curves. it should be noted that the field evidence was limited to the range of grades on the two turnpikes, namely. $310+2 \%$ on Ohio Turnpike and -3 to +3 on Pennsylvan Turnpike. In addition. Kontaratos et al (1994) disputed the analytical evidence because Dunlap et al used a sliding coefficient of friction between 0.3 to 0.5 which is 100 high according to established road safety criteria. Furthermore. Dunlap et al (1978) did not consider the casc of a vertical curve combined with the horizontal curve. 
In a more accurate model than the AASHTO point-mass formula, Kontaratos et al (1994) simulated the vehicle-road interaction using a bicycle model (Figure 2.4). The model can consider the combining effect of grade, horizontal curve, and superelevalion and the interaction between longitudinal and side fricti uns. The vehicle used in the model is a passenger car and is modelled as a body with a specific height. The variation in friction forces between rear and front axles is considered depending on the type of vehicke arive while interior and exterior tires are assumed to have equal friction forces. Assuming that the vehicle is travelling at a constant speed, the model was ured 11 determine the minimum required radius of a horizontal curve combined with an upgrade or downgrade.

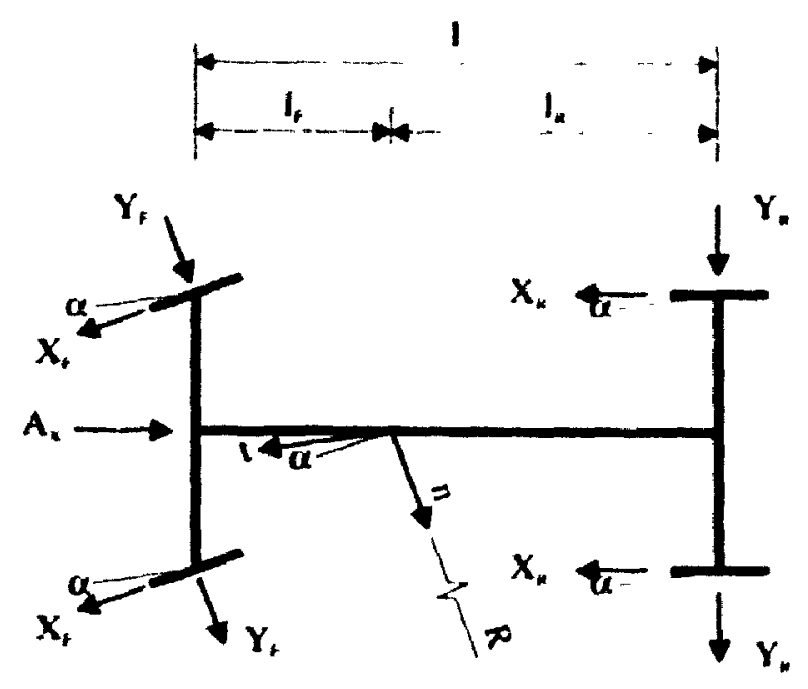

FIGURE 2.4: Forces Acting on a Passenger Car in a Bicycle Model (Kontaratos et . Il, 1994). 
The results showed that the AASHTO formula would yield conservative radii when the horizontal curve was combined with a downgrade. On the other hand, if the curve was combined with an upgrade, the required minimum radius increased with the increase of the upgrade. Depending on the design and operating speeds, there would be an upgrade biyond which the AASHTr, formula would underestimate the required horizontal curve radius. Although this may seem to contradict the intuition that downgrades are more critical than upgrades, it was explained that this would be true in the braking mode. However, in the driving mode assumed in this analysis, Kontaratos et al (1994) explained that "as the vehicle moves upgrade, greater longitudinal forces act on it, demanding greater reserves of friction. Consequently, fewer reserves of friction remain to be used in the lateral direction". An interesting observation in the results Kontaratos et al (1994) is that the effect of the upgrade tends to compromise the safety of curves designed according to the AASHTO formula when the grade is higher than $3 \%$. These grades were beyond the limits of the two turnpikes investigated by Dunlap et al (1978). Therefore, there is no contradiction between the two opposite conciusions regarding the effect of upgrades hy Dunlap et al (1978) and Kontaratos et al (1994).

Although the model by Kontaratos et al (1994) is far superior to the AASHTO point-mass formula, it should be noted that the study did not include the braking driving mode. emergency manoeuvres performed on curves, or the effect of combining vertical curves. A more complex and comprehensive vehicle dynamics model (VDM) has been developed hy Allen et al (1995). The model can simulate passenger cars, trucks, and articulated vehicles. With a total of 17 degrees of freedom (Figure 2.5), the model can 


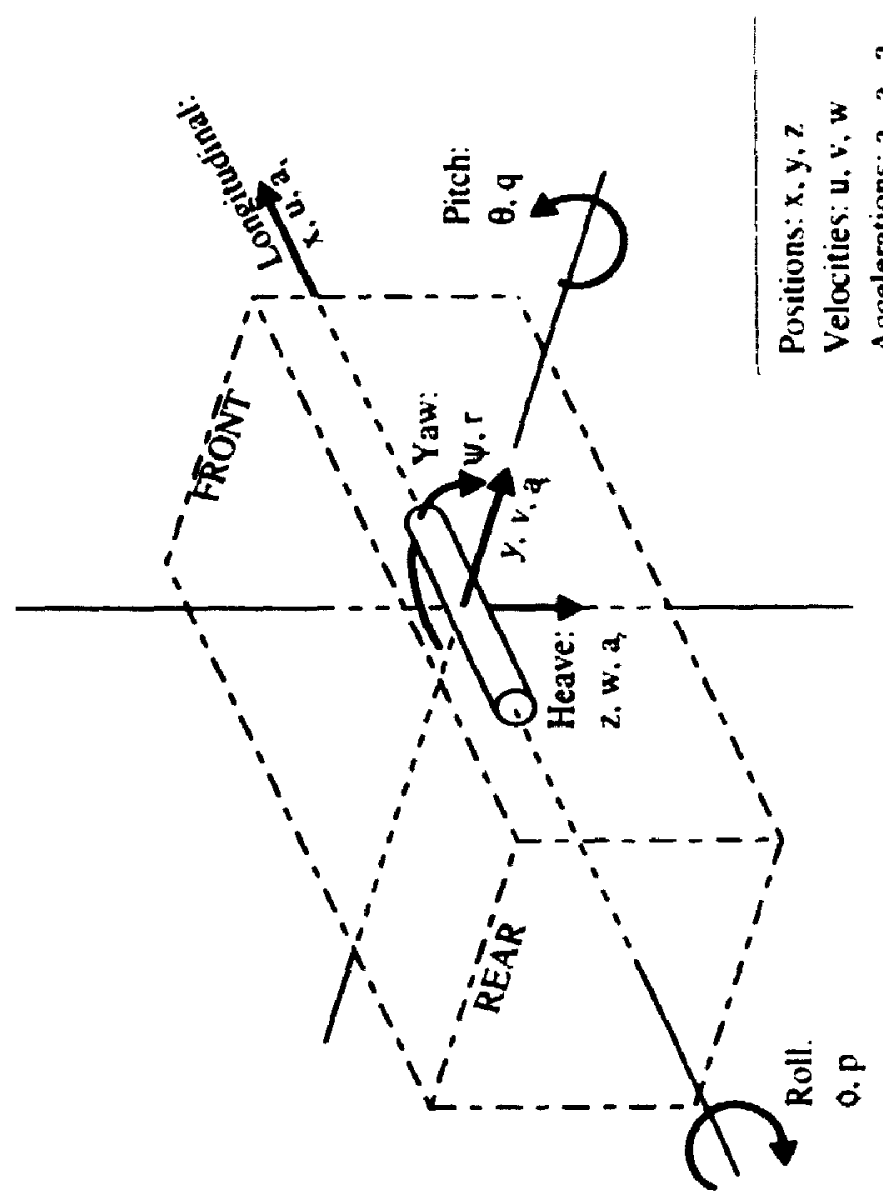

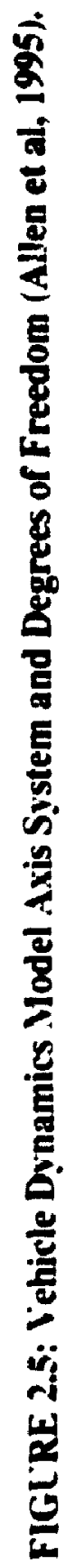

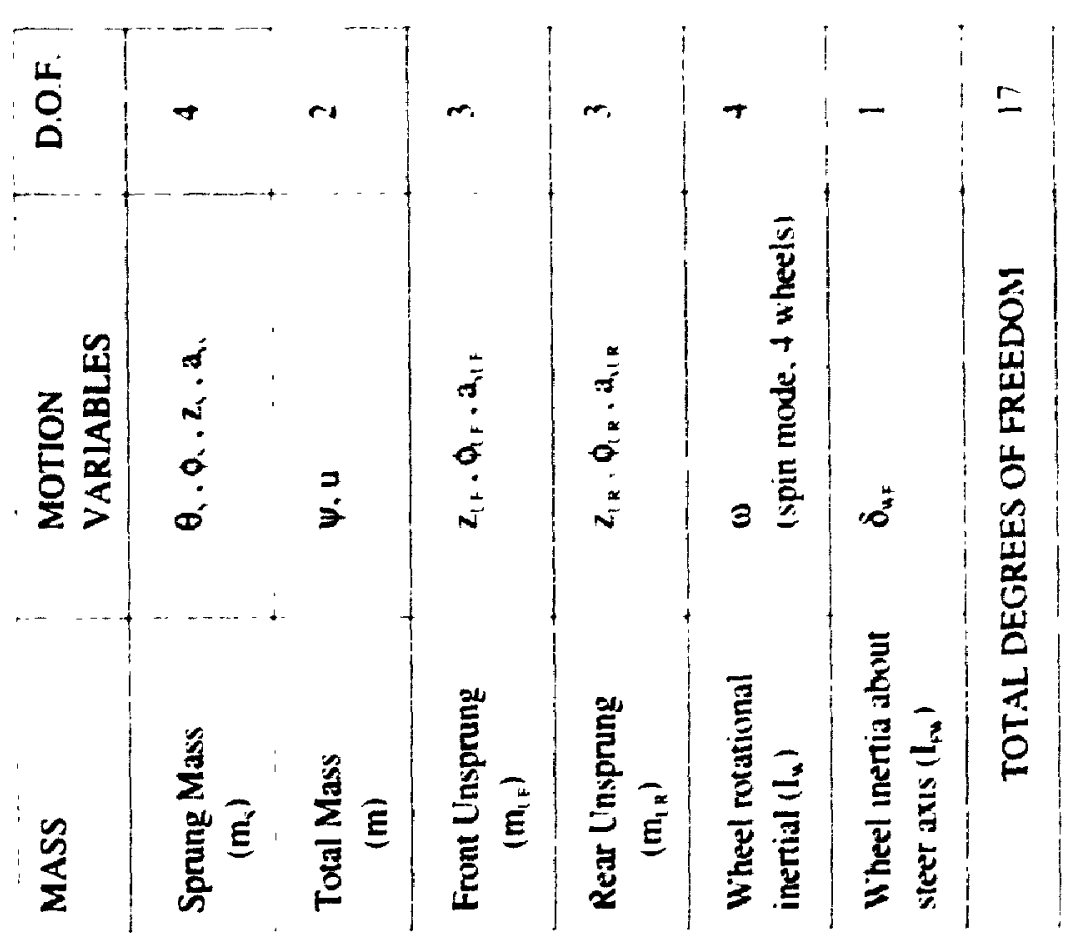


cover virtually all types of lateral, longitudinal, and angular motions. The model is also clairred to be able to simulate "virtually all driver induced maneuvering up through and including limit performance conditions defined by tire saturation characteristic (plowout, spinout) and rollover". The model has been incorporated as a main module in the Interactive Highway Safety Design Model (IHSDM) being created by the US Federal Highway Administration (Reagan, 1995). This module will permit highway designers to simulate any of the AASHTO design vehicles and drive them through the alignment. Thus, a complete speed profile, data on lateral accelerations, and the potential of accidents will be available for the designer to evaluate the available alignment alternatives (Reagan, 1994).

In summary, it can be stated that the AASHTO point-mass formula for designing horizontal curves is far from accurate and can be far from conservative (Dunlap et al, 1978). Ignoring the 3-D nature of highway alignment may lead to erroneous decisions regarding the selection of horizontal curve radii (Kontaratos et al, 1994). On the other hand, more accurate models are currently available for a more reliable alignment design based on 3-D analysis. However, more research for accurate quantification of the side frictions is still needed (Fitzpatrick, 1994; Harwood and Mason, 1994; Kontaratos et al, 1994).

\subsection{Drainage}

Drainage of storm water off the road is an integral part of highway geometric design. Water on the pavement surface reduces the available friction between the road and the 
vehicle's tires, and therefore compromises the vehicle stability and increases the risk of collisions. Moreover, high rainfall intensity combined with poor pavement surface drainage may cause complete hydroplaning where the pavement surface and the tires are separated by a thin film of water. Therefore, safe traffic operation requires highway designers to coordinate horizontal and veitical alignments and cross-sections to ensure adequate surface drainage. In addition to the pavement surface drainage, drainage facilities such as bridges, culverts, channels, curbs, and gutters are used to carry storm water across the right-of-way. The design of this latter type of drainage provisions is explained in separate manuals (AASHTO 1991; 1993), rather than being included in the AASHTO green book, and is beyond the scope of this review.

As mentioned above, poor pavement surface drainage will accunulate a significant water depth on the pavement and may lead to complete hydroplaning. However, it should be noted that the majority of wet-weather skidding accidents occurs at water depths well below that required for complete hydroplaning (Dunlap et al, 1978). For example, for a specific smooth tire and pavement surface texture, Staughton and Williams (1970) reponed that the tire brake force coefficient at $96 \mathrm{~km} / \mathrm{h}(60 \mathrm{mph})$ was less than 0.05 at a water depth of $0.762 \mathrm{~mm}(0.03 \mathrm{in})$. The corresponding water depth required for a complete hydroplaning was estimated as $3.81 \mathrm{~mm}(0.15 \mathrm{in})$. Therefore, pavement surface drainage provisions sho'ld be designed not only to avoid significant water depths which will cause complete hydroplaning but also to avoid low water depths which will jeopardize traffic safety. 
Both AASHTO and TAC design guides recommend general guidelines for pavement surface drainage. In designing the cross-section, a range of pavement crossslopes is suggested based on the pavement type. High-type two-lane highways crowned at the centre can have the lowest rate of cross-slope (1.5 to $2.0 \%$ ). As the pavement surface type gets more inferior and water can be drained more difficultly, the rate of the cross-slope should be increased. For multi-lane highways, gradual increase in the crossslope of each lane relative to the preceding one is recommended. Moreover, the advantages and disadvantages of the different cross-slope arrangements should be weighed to determine the best overall arrangement. For example, when the lanes of each travelling direction are sloped in both the median and shoulder directions, the pavement surface drainage will be more effective and less water depths will be encountered. However, drainage facilities are required to collect the water from three points (both shoulders and median). On the other extreme, if all lanes are sloped in the median direction, less expenses are required for the drainage facilities since water is collected from one point only. However, such arrangement will result in greater water depths in the inside lane which carries the traffic with higher operating speeds.

In addition to the design of the cross-slope, some caution should be practiced in designing the vertical alignment. Although level or flat grade is allowed on uncurbed highways with adequate cross-slope for drainage, a minimum grade of 0.5 (or $0.3 \%$ in case of high-type pavement) is recommended. In addition, crest and sag vertical curves should allow a minimum grade of $0.3 \%$ at a point $15 \mathrm{~m}$ away from the level point. This criterion allows for a maximum $K$ of $51 \mathrm{~m}$ per percent change of grade which 
corresponds to a maximum design speed of 80 and $100 \mathrm{~km} / \mathrm{h}$ for crest and sag curves, respectively. However, if a higher design speed is to be reached, flatter curves should be used and special attention is needed for proper pavement drainage.

Although the coordination of vertical alignments and cross-slope for adequate drainage is implicitly included in the design standards, again, a clear and quantified coordination is lacking. For example, a further enhancement of pavement drainage can be accomplished by good coordination of horizontal and vertical alignments so that $t \mathrm{t}$ : revolving points in both alignments are approximately coinciding (Smith and Lamm, 1994). As shown in Figure 2.6, by placing approximately equal-length horizontal and vertical curves at approximately the same locations, the maximum superelevation rate will coincide with the minimum longitudinal grade and vice versa. This combination would not only add to the highway aesthetics but also enhance the pavement drainage significantly. Moreover, the current design guides which base the superelevation

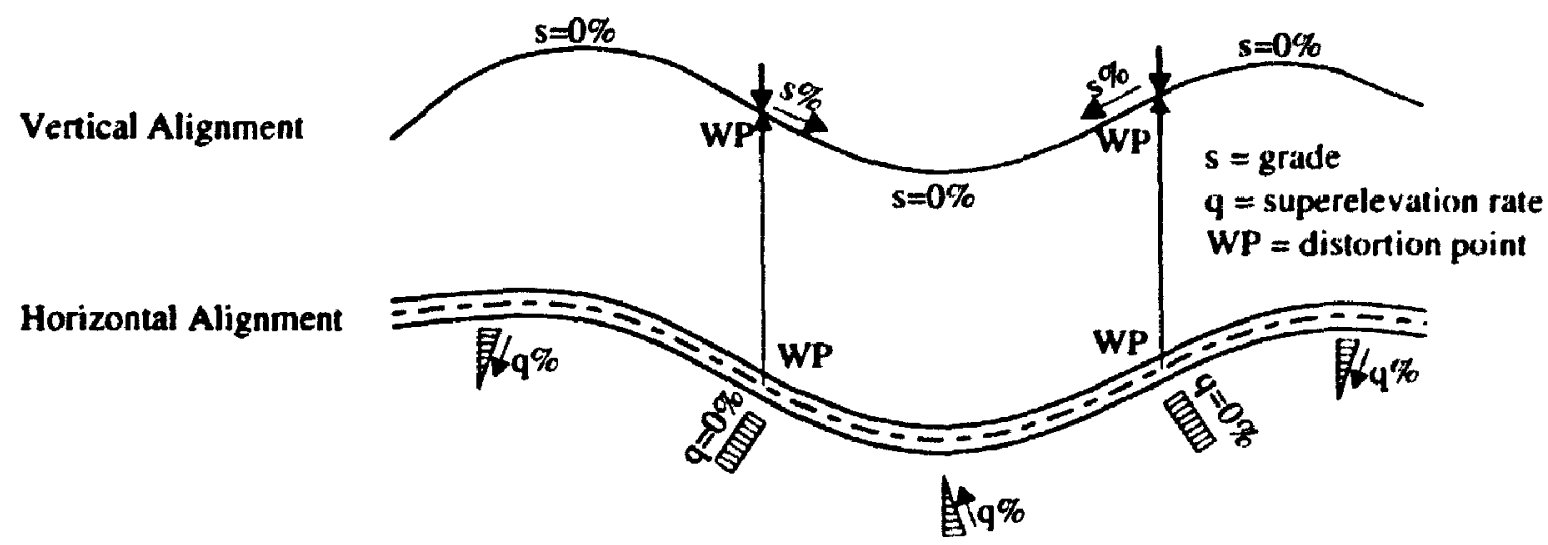

FIGURE 2.6: Coordination of Distortion Points in Horizontal and Vertical Alignments (Smith and Lamm, 1994). 
primarily on the vehicle stability aione may produce curves with long radii and low superelevation rates. With all lanes sloped in the same direction (to the inside of the curve), such a sitaation may deteriorate the pavement surface drainage significantly (Dunlap et al, 1978).

A more quantitative analysis for pavement surface drainage can be carried out using existing formulas to predict the water depth over the pavement surface (Gallaway et al, 1971; Ross and Russam, 1968; Yeager, 1971; Yeager and Miller, 1971). According to these formulas, the primary factors affecting the water depth are the road width, the superelevation (cross-slope) rate, and to a less extent, pavement surface texture. On the other hand, longitudinal grade has no or little effect. This can be explained by the fact that the grade increases the slope and the run-off length. Although the former reduces the water depth, the second increases it with a net result of almost zero (Dunlap et al, 1978). Using these formulas, Dunlap et al (1978) suggested a methodology to account for pavement drainage and the loss in friction forces on horizontal curves relative to tangent sections. Depending on the superelevation rate, rainfall intensity, drainage length, and longitudinal grade, the required increase in the pavement skid resistance on horizontal curves to overwuc the loss in friction is recommended.

Dunlap et al (1978) also investigated the correlation between pavement surface drainage and traffic safety. In a comprehensive investigation of a $1^{\circ}$ horizontal curve $(R=1746 \mathrm{~m}$ ) on Ohio Tumpike with significantly high accident rate, it was found that the wet-weather accidents were over-represented. The percentage of wet-weather accidents represented 79 and $62 \%$ of the total accidents when the pavement was made of portland 
cement and asphalt cement, respectively. Observing that the superelevaltion rate was relatively low $(1.56 \%)$ which was equal to the crown slope on the tangent, the waller depth on the curved section was predicted to be almost twice that on the tangent. Thus. the curve experienced a considerable sudden loss in the available friction. Subsequently, the safe operating speed on the curve was significantly less than that on the tangent. Although drivers expect a lower safe operating speed on curves than on tangents, such a sudden and significant drop in the safe operating speed was heyond their expectalions, and therefore they failed to adjust their speed properly.

In summary, it can be seen that the current design standards include implicit correlation between vertical alignment and cross-section in providing proper pavement surface drainage. However, a clear coordination is not included explicitly. Also, the current provisions of the superelevation rate can be misinterpreted to produce unsatic horizontal curves during wet weather. Further research is still needed to establish a clear correlation between pavement surface drainage and traffic safety. Quantitative analysis and guidelines for the effect of combined alignments on providing proper pavenuent surface drainage are still needed.

\subsection{Highway Aesthetics}

Although highways' essential function is to provide safe and rapid mobility for goxds and people, their pleasing appearance should not be overlooked. The interrelationship between highway aesthetics and traffic safety has been emphasized (ASCI: 1977; Sinith and Lamm, 1994); though has not been well quantified. Poor coordination of horizontal and 
vertical alignment may violate the driver expectations or cause an erroneous perception of information. For example, in viewing existing highways from the driver's point of view, Mori et al (1995) concluded that the alignment coordination may cause the drivers to perceive the alignment wrongly. Such a wrong perception may be hazardous if the driver perceives a sharp curve as a flat curve. Therefore, the AASHTO green book emphasizes the need for a comprehensive study of the alignment coordination during the design phase to avoid the excessive costs associated with any deficiency that may be noticed later.

However, unfortunately. "the exact adherence to preceding (specific) design standards does not guarantee obtaining a satisfactory and aesthetically pleasing design" (Easa, 1995). This statement summarizes, in simple terms, a widely spread conviction among highway professionals and researchers. The main reason for this conviction is the current design practice ir, which each of the horizontal and vertical alignments are designed separately. Since the drivers do not view the highway in 2-D but rather in 3-D. there is no guarantee that the resulting combined alignment will. be satisfactory. Moreover, in designing either alignment, both AASHTO and TAC design guides pay attention to a single element (curve or tangent) at a time. Such practice may cause further violations to highway aesthetics and design consistency (Lamm and Smith, 1994).

In order to achieve a better coordination between horizontal and vertical alignments, AASHTO and TAC provide general guidelines which should enhance the aesthetics of the combined alignment. In following these guidelines, the designer must depend on his/her ability to visualize the highway in 3-D using the 2-D drawings of the 
plan and profile. Such a task is believed to be achievable by an experieiked designer. Yet, the AASHTO design guide recommends using physical models in complex situations where using the 2-D maps for 3-D visualization may be difficult.

As an easier alternative for 3-D highway visaalization, computer simulation models can be used. This computerized visualization has been approached as early as in the 1960's (Geissler, 1968; Park et al, 1968). With the recent advancements in computers, the simulation and visualization of 3-D highway alignments have been much easier and more accurate using computer-aided drafting and design package's (C AI)(S). Currently, many highway agencies in the US and Canada, governmental and private, are using 3-D computer visualization in reviewing the highway alignment before construction or reconstruction (Larson, 1996). Taking the time as a fourth dimension to create short movies, the available packages are also used to generate 4-D visualization to the highway from the driver's point of view. This 3-D and 4-D visualizaltion can help detect any alignment deficiencies before it is too late or too expensive to fix them. Moreover, it is proved to be a fast, understandable, and relatively inexpensive way for highway agencies to communicate with the public. 3-D visualization is also a main module in the IHSISM being created by the US Federal Highway Administration (Reagan, 1994).

Still, however, further research is needed for good quantification of the effect of highway aesthetics on traffic safety. Similarly, research on the effect of alignment coordination on the driver's perception of information is needed. Under the heading " $A$ n Interesting Phenomenon", Smith and Lamm (1994) mentions a specific case of alignment coordination that needs further research. This case is the driver's perception of horizontal 
curves when superimposed with sag vertical curves (Figure 2.7). Using CADD, the hypothesis that sag vertical curves cause horizontal curves to appear flatter than what they really are can be examined. Such an erroneous perception of information would cause drivers to travel at higher speeds than what they should ajopt, and, subsequently, would increase the risk of collisions.

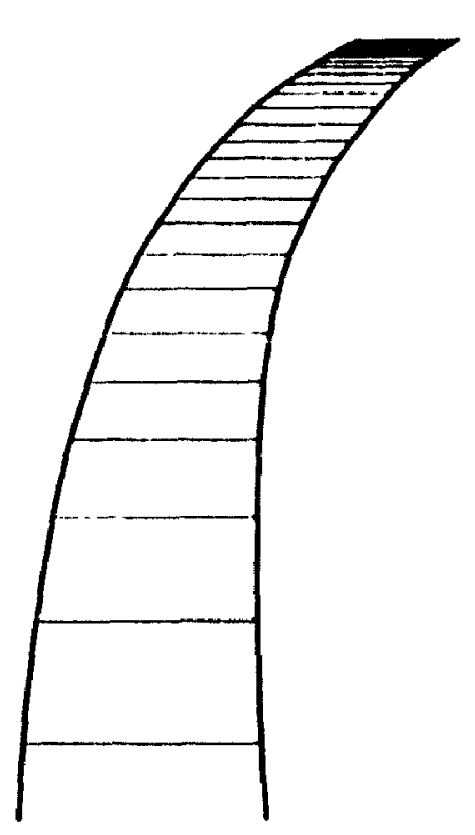

(a) Horizontal Curve

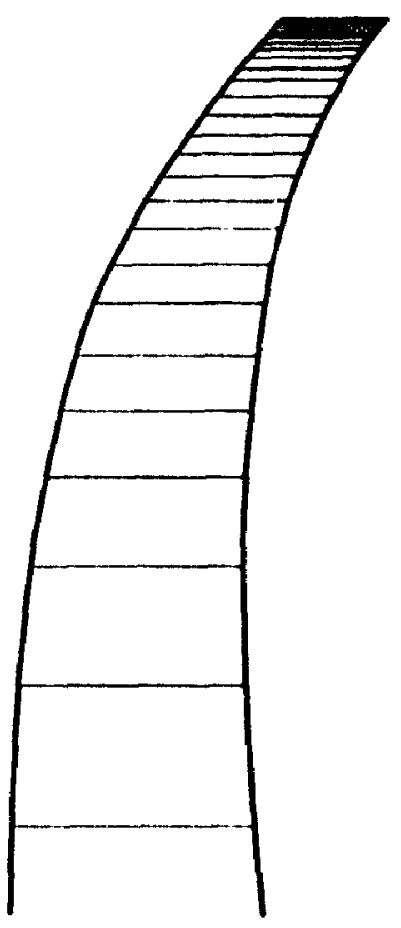

(b) Horizontal Curve with Superimposed Sag Vertical Curve

FIGURE 2.7: Efrect of Superimposed Sag Vertical Curves on Appearance of Horizontal Curves (Smith and Lamm, 1994). 


\subsection{Summary of Review}

To sum up. the previous review shows clearly that the current highway geometric design standards are based entirely on 2-D separate alignments. Moreover, in the 2-1) allignment. only a single element is looked at. separately from the other a...gunnemt compruneents. Although analytical models are currently available for a better consideration of more complex 2-D alignments, they have not been included in the design standards. The 3-1) analysis of combined highway alignments, however, has been thought to le complex and cumbersome. As a result, not much research work has been directed all designing highways in 3-D alignments or quantifying the effect of ignoring the 3-1) natture of highway alignments. Consequently. the margin of safely or risk on highways destgned according to the current standards cannot be quantified. Therefore. the highway community agrees that a considerable research work is needed to establish 3-1) highway geometric design standards. As stated by Krammes and (jarnham ( (9)5) "Se-verat issuers

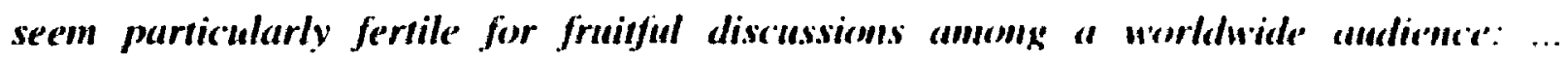
Considering the interrelationships among horizontal and vertical alignment and roudway cross section". 


\section{CHAPTER 3}

\section{MODFLLING REQUIRED PASSING SIGHT \\ DISTANCE AND PASSING ZONES}

In this Chapter, based on the examination of the current design and marking practices and existing research work related to the required PSD and based on the critique of the available models presented below, a model is developed to simulate the entire passing manoxuvre (Hassan. et al 1996a: 1995a). In addition to the required PSD. the model can be used to determine the location and the minimum length of passing zones. The mechanism of the passing manocurre upon which the model is based is presented first. Then, the values of the required PSD using this model, referred to as the revised model, are validated using field measurements made by Van Valkenberg and Michael (1971). Finally. practical design considerations for PSD and passing zones are discussed.

\subsection{Critique of Existing Models for PSD}

\section{t.I.I Gilennon's Model}

Referring to Chapter 2. two parameters in Glennon's model are worth of closer investigation. The first parameter is the clearance, $C$. between the passing and opposing vehiciles at the end of the pass. Although the concept of the point of no return is basically the same as that of the critical position, Van Valkenberg and Michael (1971) identified the point of no return by producing the same safefy factor whether the pass is completed or aboned while Lieberman (1982) identified the critical position by producing 
the same clearance for completed and athorted passess. In the completed pass. the passing vehicle will maintain its speed, 1 , while decelerating in abrrted passes, and thus, hatring a final speed lower than $1:$ Therefore, if the clearance distance is the samte in moth caises. the clearance headway will be greater in aborted passes than that in completed passes. Undoubtedly, the safety factor depends on the time headway not on the clearanse distance. For example, two stationary vehicles will maintain an infinite sattely lactur ceven if the clearance between them is almost zero because the lime headway in thus cane is infinity. Therefore, the definition given by Van Valkenberg and Michatel (1971), and interpreted by Glennon (1988) by assuming a minimum acceptable headway le:tween the two vehicles at the end of the pass, appears to be more reasonathle. However, II lins model's derivation, Glennon (1988) considered that the clearance ( is collnstant for completed and aborted passes. This of course is in disagrectment will the more reasonable definition for the sritical position and is interpetation by a mumum acceptable headway. Generally, if two vehicles are travelling in opposile directuons with speeds $v_{1}$ and $v_{2}$. the clearance $C$ which makes them reach the salme primt afler a leadwaty $h$ will be $\left(v_{1}+v_{2}\right) h$.

The second parameter is the gap, $G$, between the passing and innpeding velucles at the end of the pass. In the model's derivation, Glennon (I9KX) balted "assumming a minimum acceptable headway of one second for $G$, then $G=m "$, where $m$ is the differential speed and is equal to the difference between the speed of the impeding velucke and the design speed. Although this equality may theoretically be true ill somke yocial cases, it seems very unrealistic. As shown in figure 3.1. knowing that the total limle 
required $w$ ahort the pass is $t_{2}+1 \mathrm{sec}$ (for perception-reaction time) and the time required to complete the pass is $\ell_{1}$, for the value of $G$ to equal $m$ (times $1 \mathrm{sec}$ ), one has to assume:

1. For a completed pass, at time $t=t_{1}-1$, the rear bumper of the passing vehicle is abreast of the front bumper of the impeding vehicle. Then, from this position, the driver of the passing vehicle will initiate the lateral shift of one lane widh, to return back to the right lane, and complete it in $1 \mathrm{sec}$.

2. For an aborted pass, at time $t=t_{2}$, the rear bumper of the impeding vehicle is abreast of the front bumper of the passing vehicle and the passing vehicle will travel at a constant speed of $(v-2 m)$ for the remaining second (note that the speed of the impeding vehicle is $v-m$ ). Also, the driver of the passing vehicle will initiate the lateral shift from this position and complete : in 1 sec. Another possible scenario for an ahorted pass is as follows: the passing vehicle will continue decelerating during the entire time period $t_{2}$. Obviously, $G$, in this case, cannot be related to $m$.
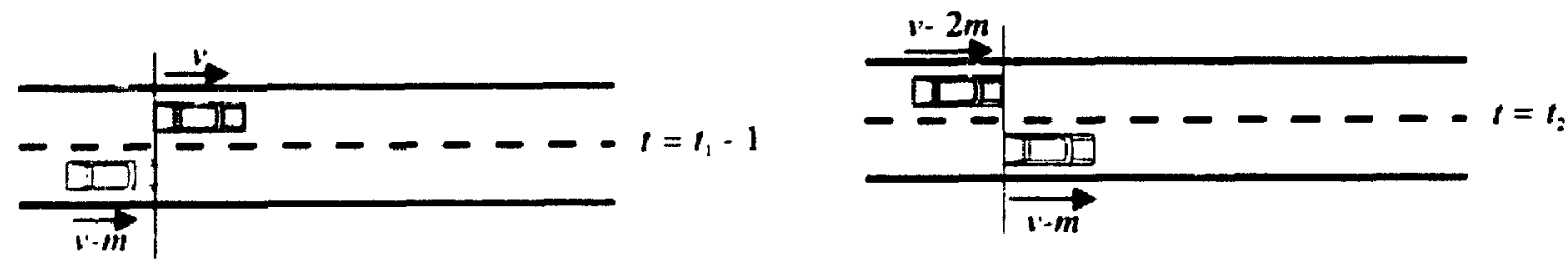

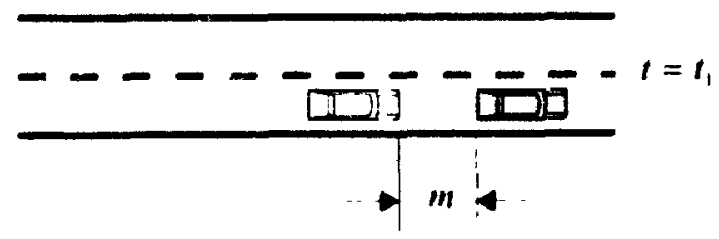

(a) Completed Pass

בassing Vehicle

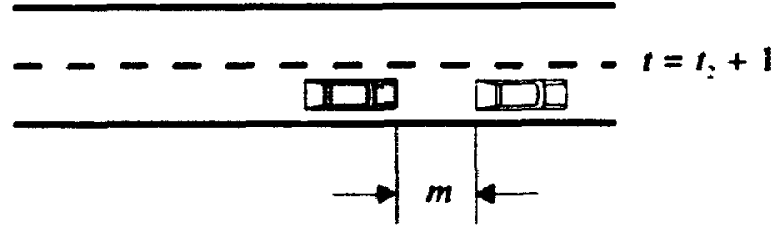

(b) Aborted Pass

FIGURE 3.1: Assumptions Required for $G=m$. 
The above assumptions are extremely difficult to justify or accept. It is obvious that initiating the lateral shift from the positions stated in both assumptions is very hazardous. Actually, the driver of the passing vehicle will maintain some gap distance between his/her vehicle and the impeding vehicle before starting shifting to the right lank. Subsequently, for $G$ to equal $m$, the driver has to complete the lateral shift in a time much less than a second.

Moreover, as defined in the HCM (1994), the headway is the time hetween successive vehicles as they pass a point on a lane or roadway. The headway, as stated in the HCM (1994), can be measured using stopwatch observations as vehicles pass a point on the roadway. As shown in Figure 3.2, if an ohserver measures the time spacing between the passage of the passing and impeding vehicles at the end of a completed pass, it will be $m /(v-m)$ which is much lower than 1 sec for speeds higher than $2 m$. For an aborted pass, the time spacing will be $m / v_{f}$, where $v_{f}$ is the final speed of the passing vehicle due to deceleration. Although the time spacing in this latter case is greater than the corresponding time for a completed pass, it will remain less than the one second minimum acceptable headway, assumed by Glennon (1988), for high speeds where $v_{f}>$ $m$. The physical interpretation of such very low headway is that the driver of the trailing vehicle will not have any opportunity to decelerate if the leading vehicle stops suddenly unless his/her perception-reaction time approaches zero. As shown in Figure 3.3, a more reasonable gap distance, $G$, between two vehicles having different speeds, $v_{1}$ and $\ddot{v}_{2}$, where $v_{1}>v_{2}$ and $v_{1}$ is the speed of the leading vehicle, and for a headway of $h$ 


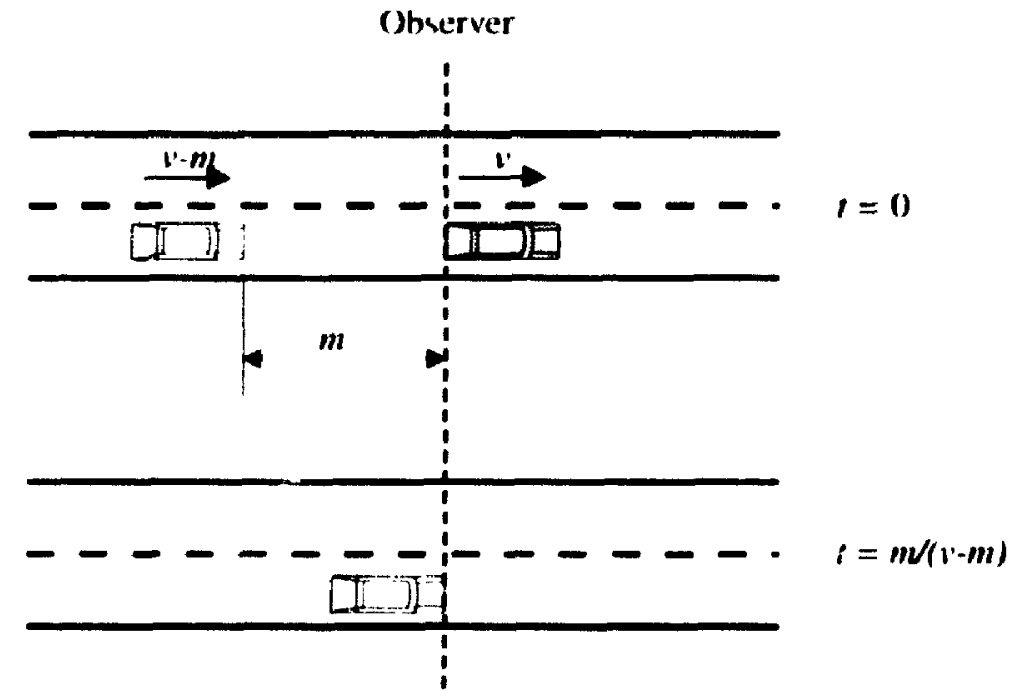

FIGURE 3.2 : Actual Headway at the End of a Completed Pass.

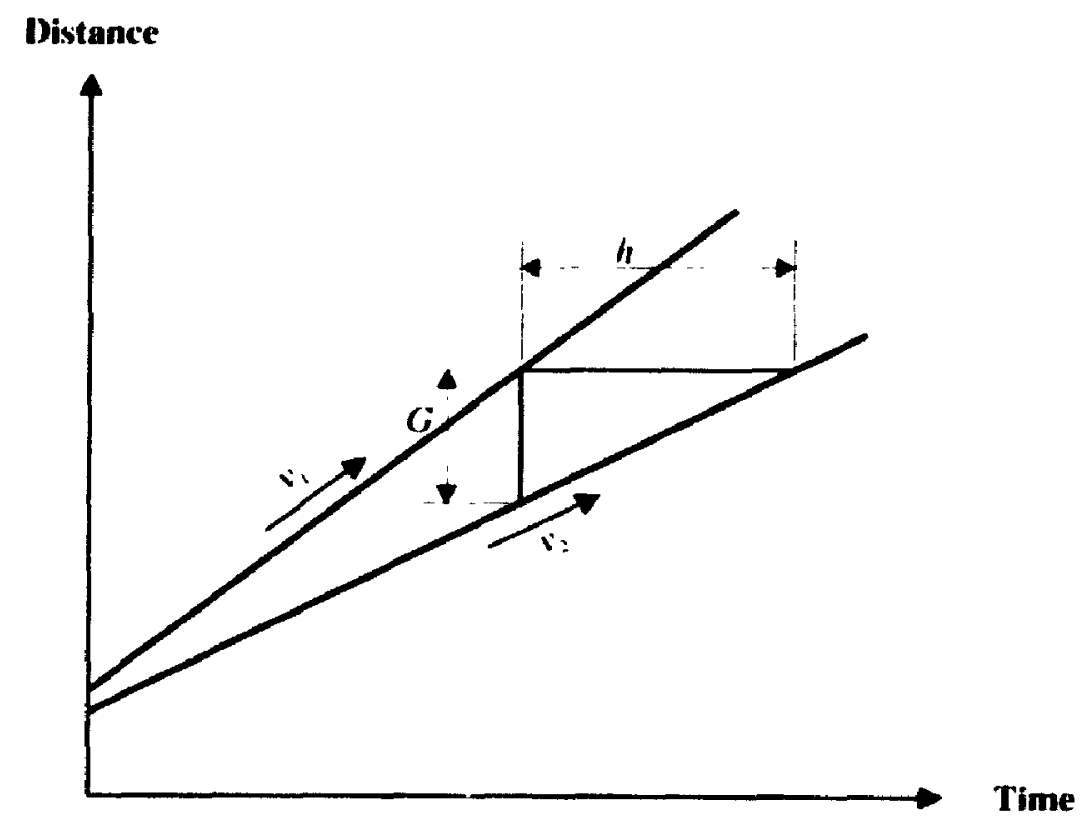

FI(iURE 3.3: Time-Space Relationship for Two Vehicles with Speeds $v_{1}$ and $v_{2}$. 
seconds, is $v_{2} h$. Therefore. the values of $C$ and $G$ in the model should be hased on the speeds of the vehicles involved.

\section{I.2 Rillet et al's Model}

A closer inspection of the modified model by Rillet et al (1990) reveals the following:

1. In develop. ing the model, Rillet et al (1990) stated "a correct appronch would be "1" multiply the time headway by the speed of the stewer moning vehicle. (1-m)". However, since the passing vehicle in aborted passes decelerates to a speed lower than the speed of the impeding vehicle, $\left(v^{-}-m\right)$ in this case will correspond to the fister vehicle. Therefore. a more correct approach would be to multiply the time heidwaly by the speed of the trailing vehicle.

2. The assumption of a minimum terminal speed appears to be too conservative. In a study conducted by TAC, Good et al (1991) stated "it serems. illegical to cassume thut drivers. having determined that a siti atton exists in which there is polential for an

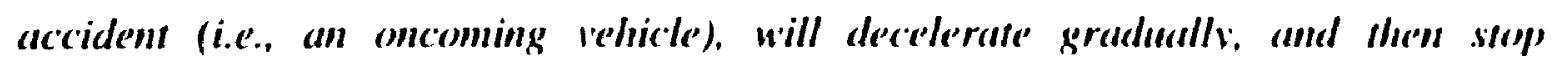
decelerating even though they are not in a position to re-integrate themselves into the traffic stream".

3. This conservative approach in considering the aborted passes pushes the critical position back, i.e. closer to the beginning of the pass, as the design speed increases. Consequently, the possibility of having the passing vehicle reached the critical position while still accelerating increases. This explains the observation "ther 
acceleration at the critical point is still occurring at design speeds of up to $100 \mathrm{~km} / \mathrm{h}$ when the impeding vehicle is a car (5 m long)" (Rillet et al, 1990).

4. Although the model successfully considered the acceleration occurring at and beyond the critical position in completed passes, it failed to consider the same event in aborted passes. Instead, the speed of the passing vehicle during the perceptionreaction time in aborted passes was taken constant (Rillet et al, 1990).

Based on the above discussion, a revision for the models of required PSD is needed. A revised model based on the mechanism of the passing manoeuvre, explained in the following section, is developed in the subsequent sections.

\subsection{Mechanism of the Passing Manoeuvre}

An ideal passing manoeuvre should proceed as follows:

First. the manoeuvre is initiated as follows:

- The impeding and opposing vehicles are travelling at constant speeds of $v-m$ and $v$, respectively, during the entire manoeuvre.

- At the beginning of the pass, the passing vehicle is trailing the impeding vehicle and travelling at a speed of $v-m$. A minimum headway, $h$, is maintained between the front bumper of the passing vehicle and the rear bumper of the impeding vehicle.

- Then, the passing vehicle accelerates with a constant rate, $a$, to a speed $v$ while shifting to the left lane. The sight distance required at this stage is minimal and corresponds to aborting the pass safely. 
- As the pass builds up. the sight distance required for the passing vehicle to ahort the pass increases and that required to complete the pass decreases.

Second, if the manoeuvre cannot be completed safely, it should be aborted as follows:

- If, at any instance, the driver of the passing vehicle decides to abort the pass, a minimum headway, $h_{l}$, should be maintained between the front humper of the passing vehicle and the rear bumper of the impeding vehicle. Similarly, a minimum headway. $h_{o}$. should be maintained between the front bumper of the passing vehicle and the front bumper of the opposing vehicle.

- In aborting the pass, the driver of the passing vehicle takes a perception-reaction time. $P$. before applying the brakes. During this perception-reaction time, the speed profile of the passing vehicle is assumed not to be intluenced by the need to abort the pass. This means that the passing vehicle will continue its acceleration until it reaches the design speed, $v$, or to the end of $P$, whichever is first.

- Then, the vehicle keeps decelerating with a constant rate, $d$, until it is hack in the right lane.

Finally, at a certain point, the critical position, the sight distance required to abort the pass equals that required to complete it. The sight distance at this point is called the critical sight distance.

- By passing the critical position, the passing vehicle can complete the pass safely.

- At the end of the completed pass, the minimum headways, $h_{o}$ and $h_{l}$. should be maintained between the front bumpers of the passing and opposing vehicles and 
between the rear bumper of the passing vehicle and the front bumper of the impeding vehicle, respectively.

The model derivation, presented in the following sections, includes the determination of the critical position, the critical sight distance, the minimum length of passing zones and the profile of the required sight distance with the evolvement of the pass.

\subsection{Critical Position and Critical Sight Distance}

Figure 3.4 shows the time-space diagram for a completed and an aborted pass beginning from the critical position. Referring to the completed pass,

$$
\Delta_{1}+x_{1}+v t_{1}=L_{p}+G_{1}+(v-m)\left(t_{\mathrm{d}}+t_{1}\right)
$$

or

$$
\Delta_{a}=L_{p}+G_{1}-x_{1}+(1-m) t_{a}-m t_{1}
$$

where

$L_{P}=$ length of passing vehicle,

$t_{1}=$ time required to complete the pass after accelerating to the speed $v$,

$G_{1}=$ distance between the rear bumper of the passing vehicle and the front bumper of the impeding vehicle at the end of a completed pass,

$t_{u}=$ time required to complete the acceleration from the critical position,

$x_{1}=$ distance travelled by the passing vehicle during the time $t_{a}$, and 


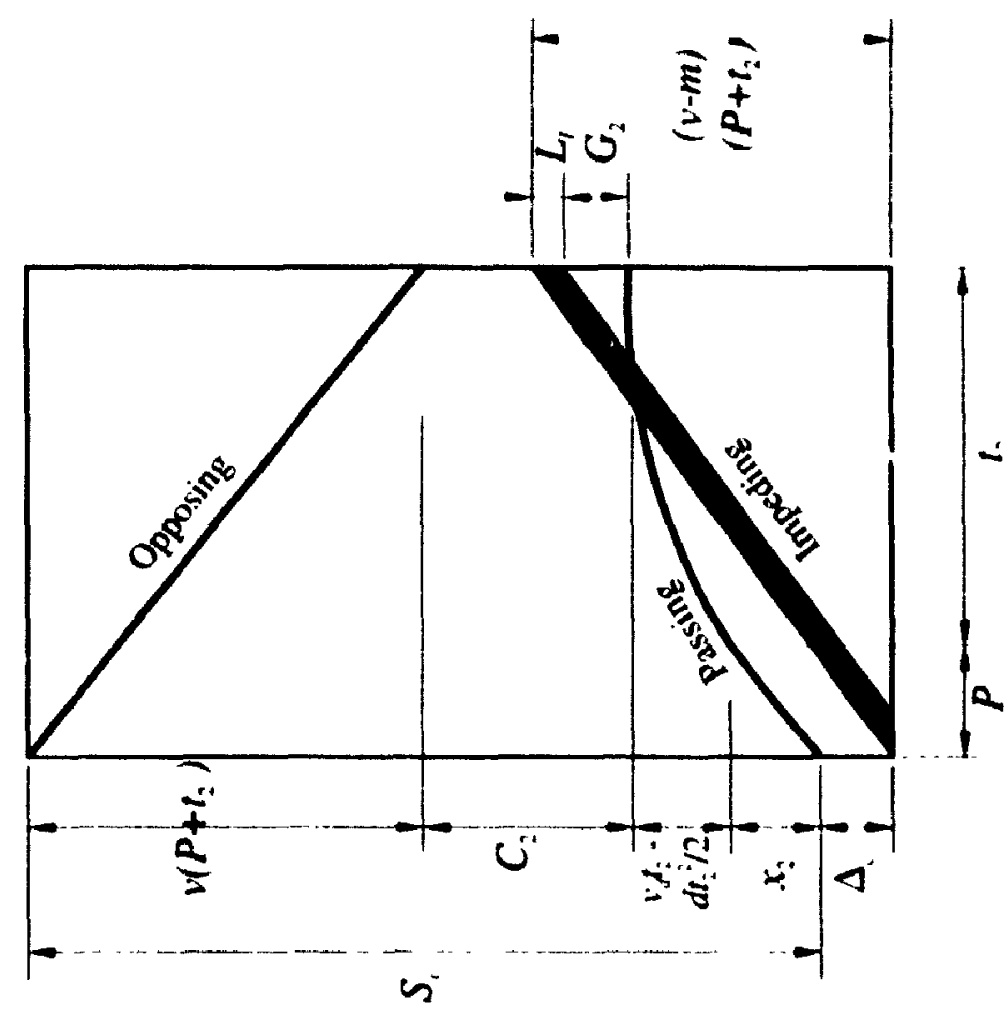

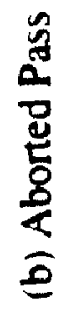

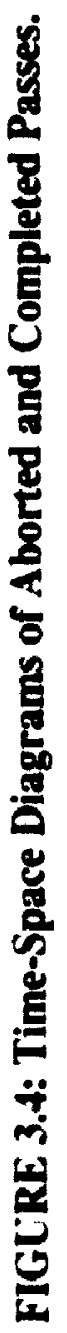

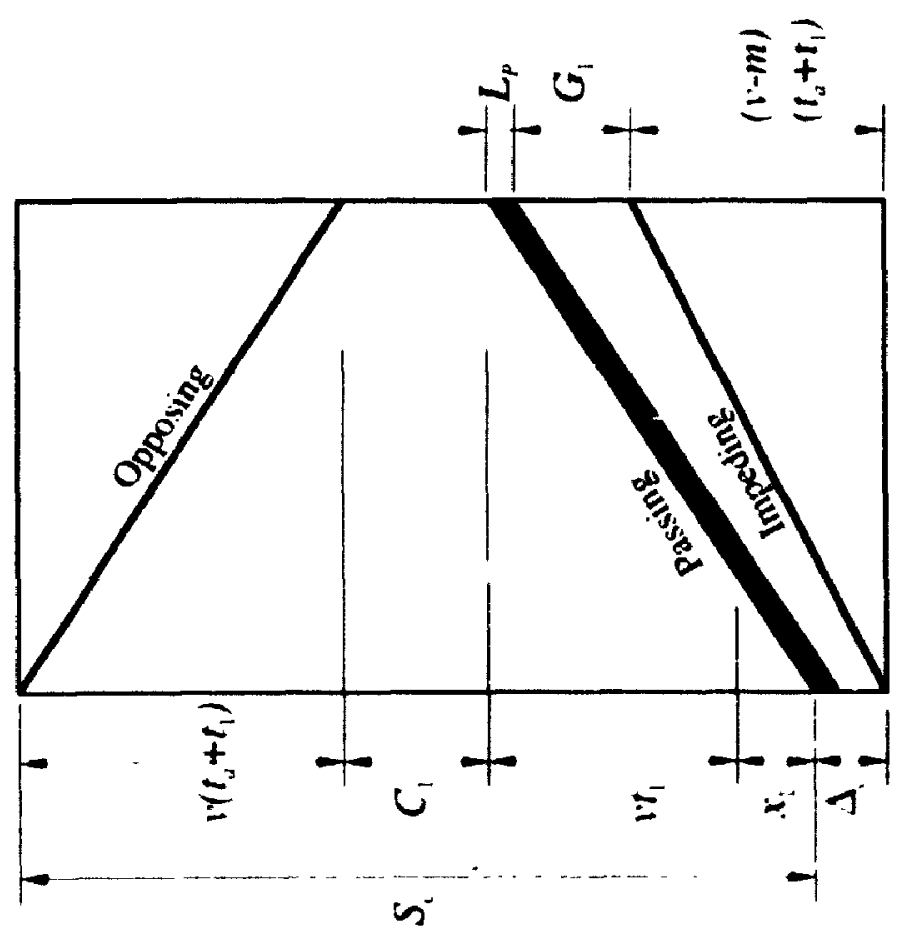


$\Delta_{\mathrm{t}}=$ distance between the front bumpers of the impeding and passing vehicles at the critical position.

Similarly, for an aborted pass,

$$
\Delta_{1}+v_{2}+v_{1} t_{2}-\frac{d t_{2}^{2}}{2}=(1 \cdots m)\left(P+t_{2}\right)-L_{1}-G_{2}
$$

or

$$
\Lambda_{1}=\frac{d l_{2}^{2}}{2}+\left(1-m-1_{1}\right) t_{2}+(1-m) P-L_{1}-G_{2}-x_{2}
$$

where

$L_{1}=$ length of the impeding vehicle,

I = perception-reaction time,

$t_{z}=$ time required 10 abort the pass from the critical position (after the perception-reaction time).

$G_{2}=$ distance between the front bumper of the passing vehicle and the rear bumper of the impeding vehicle at the end of an aborted pass.

$v_{d}=$ speed of the passing vehicle after the perception-reaction time, and

$r=$ distance travelled by the passing vehicle during the perception-reaction time.

By equaling $\Delta_{1}$ in Equations 3.2 and 3.4 .

$$
t_{1}=\frac{L_{1}+I_{i}-x_{1}+x_{2}+(v-m)\left(t_{a}-P\right)+G_{1}+G_{2}-\left(v-m-v_{d}\right) t_{2}-d t_{2}^{2} / 2}{m}
$$

By equating the critical sight distance, $S_{\imath}$, for the completed and aborted passes, 


$$
v\left(2 t_{1}+t_{1}\right)+x_{1}+C_{1}-v\left(P+t_{2}\right)+x_{2}+v_{1} t_{2}-\frac{d t_{2}}{2}+c_{2}
$$

or

$$
t_{1}=\frac{v\left(P-t_{a}\right)+x_{2}-x_{1}+C_{2}-C_{1}+\left(v+v_{d}\right) t_{2}-d t_{2}^{2} / 2}{2 v}
$$

where

$C_{1}=$ distance between the front humpers of the passing and opposing vehicles at the end of a completed pass, and

$C_{2}=$ distance between the front bumpers of the passing and opposing velicles at the end of an aborted pass.

As explained earlier and knowing that the speed of the passing vehicle at the end of an aborted pass equals $\left(v_{t}-d t_{2}\right)$, the values of $G_{1}, G_{2}, C_{1}$, and $C_{2}$ will be as follows:

$$
\begin{aligned}
& G_{1}=(v-m) h_{1} \\
& G_{2}=\left(v_{t}-d t_{2}\right) h_{l} \\
& C_{1}=2 v h_{o} \\
& C_{2}=\left(v+v_{d}-d t_{2}\right) h_{o}
\end{aligned}
$$

From Equations 3.5 and 3.7 and substituting for the values $, i f G_{1}, G_{,}, C_{1}$, and $C_{2}$.

$$
\begin{aligned}
& l_{2}^{2}\left[\frac{d(2 v-m)}{4 v m}\right]_{t}+t_{2}\left[\frac{v+v_{d}-d h_{o}}{2 v}+\frac{v-m-v_{d}+d h_{1}}{m}\right]+ \\
& {\left[\frac{v_{d} h_{o}-v\left(t_{a}-\beta+h_{o}\right)+x_{2}-x_{1}}{2 v}-\frac{L_{p}+L_{1}+(v-m)\left(t_{d}-P+h_{1}\right)+v_{d} h_{1}+x_{2}-x_{1}}{m}\right]}
\end{aligned}
$$

which can be solved to get the value of $t_{2}$. Then, the values of $t_{1}$ and $\Delta$, can be determined using the previous equations. Finally, $S$, can be formulated as: 


$$
S_{1}=v\left(2 t_{1}+t_{a}+2 h_{0}\right)+x_{1}
$$

However, to determine the distances $x_{1}$ and $x_{2}$ in the previous equations, the speed of the passing vehicle at the critical position, $v_{c}$, must be known. 'Therefore, an iterative procedure can be followed as follows:

1. Assume an initial value of $v_{1}$ as the design speed $v$. In this case, $t_{a}$ and $x_{1}$ will vanish and $v_{d}$ and $x_{2}$ will be equal to $v$ and $v P$, respectively.

2. Use the model to determine $\Delta_{\imath}$.

3. Knowing the acceleration rate of the passing vehicle, $a$, determine the time $t_{c}$ required for accelerating from the speed $(v-m)$ to the speed $v_{1}$ as:

$$
t_{1 .}=\frac{v_{1}-(v-m)}{a}
$$

4. Determine the time $t_{a}$ during which the passing vehicle is travelling with a speed equal to or greater than $v$, before reaching the critical position. Referring to Figure $3.5, t_{1,}$ can be calculated as:

$$
t_{11}=\frac{\Delta_{1}+L_{1}+(v-m) h-a t_{1}^{2} / 2}{v_{1}-(v-m)}
$$

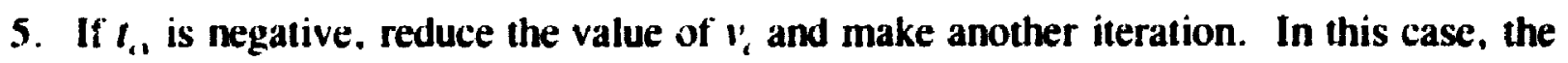
values of $t_{d,}, x_{1}, x_{2}$, and $v_{d}$ will be as follows:

$$
t_{a}=\frac{v-v_{i}}{a}
$$




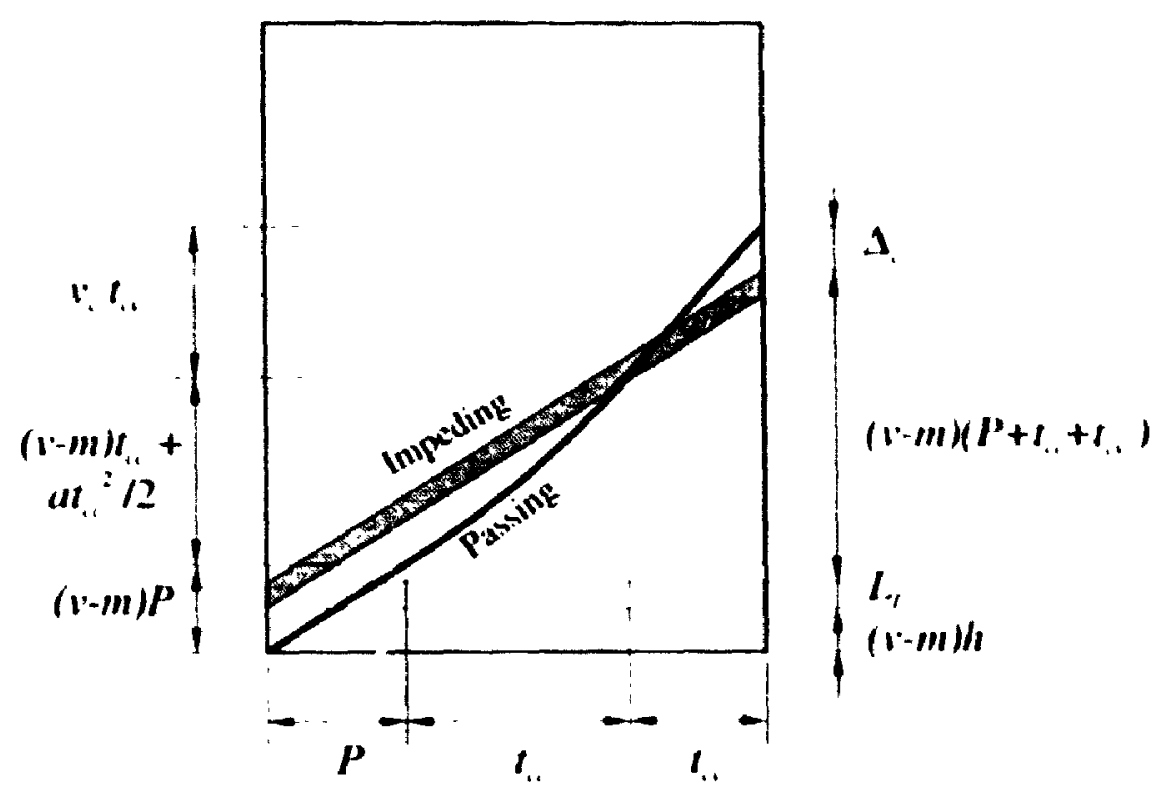

FIGURE 3.5: Time-Space Diagram for the Passing Manceuvre Between Puss Initiation and Critical Position.

$$
\begin{aligned}
& x_{1}=v_{c} t_{a}+\frac{a t_{a}^{2}}{2} \\
& x_{2}= \begin{cases}v_{c} P+\frac{a P^{2}}{2} & t_{a} \geq P \\
v_{c} t_{a}+\frac{a t_{a}^{2}}{2}+v\left(P-t_{a}\right) & t_{a}<P\end{cases} \\
& v_{d}= \begin{cases}v_{c}+a P & t_{d}>P \\
v & t_{a} \leq P\end{cases}
\end{aligned}
$$

6. Iterations should end if $t_{w} \geq 0$ and $v_{\mathrm{c}}=v$ or $t_{\mathrm{v}}=0$ and $v_{\mathrm{q}}<v$.

The solution of the previous equations may produce a positive value of $\Delta$, which means that the passing vehicle is ahead of the impeding vehicle. This can be interpreted 
as that a salie passing manceuvre may, in some situations, require the driver of the passing vehicle to abort the pass after being ahead of the impeding vehicle. Praclically. drivers should not he expected (1) abide to such a requirement. Therefore, it is recommended to provide the passing vehicle with the sight distance required to complet the pass when its iront bumper is abreast of the front bumper of the impeding vehicle, i.e., at $\Lambda=0$ at most. where $\Delta$ is the distance between the front humpers of the impeding and the passing vehicles. Substituting for $\Delta_{c}=0$ in Equation 3.2. the time required to complete the pass in this casce, $t_{1}$, can be calculated as follows:

$$
l_{i}^{*}=\frac{(1-m)\left(h_{0}+l_{1,}\right)+L_{p}-x_{1}^{*}}{m}
$$

where

$$
\begin{aligned}
& i_{1}=\text { time required to complete the acceleration from the critical position. and } \\
& r_{i}=\text { distance travelled by the passing vehicle during the tinke } i_{u} \text { - } \\
& i_{a}^{*} \text { and } x_{i}^{*} \text { can be determined using Equations } 3.10 \text { to } 3.13 \text {. }
\end{aligned}
$$

The'l, Equallion 3.9 can be rewritten as follons:

$$
S_{1}= \begin{cases}v\left(2 t_{1}+t_{1}+2 t_{0}\right)+r_{1} & \Delta \leq 0 \\ v\left(2 t_{1}+t_{1}+2 t_{1}\right)+t_{1} & \Delta>0\end{cases}
$$

It should be noted that although $L_{t}$ and $L_{l}$ are not explicit parameters in the formula for $S_{1}$. they affeet the values of $t_{1}, t_{3}, \Delta_{1}$. and in turn $S_{4}$. 


\subsection{Minimum Length of Passing Zone}

The models based on the concept of ne critical sight distance, if inling the monkel developed in this research. determine the required stght distance at the critical penstient. However, it is obvious that the passing manceure should be immtated at certain tume and distance before reaching the critical position. Theretore, the destance navelled by the passing vehicle from the beginning of the pass until reaching the critleal pestlem. $I_{\text {. }}$ would represent we absolute minimum length of the passing conce required tor silfie patsses. This length would guarantec that at least onc pass can the initialed on the passtng conc and reach the critical position betiore the passing fone conds. The time, 1, required hor the

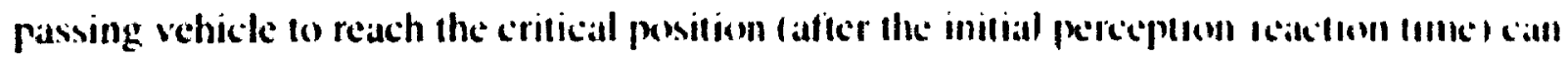
be calculated by adding the times $t_{1,}$ and $t_{1,}$ from Equations 3.10 and 3.11. Whe't $t_{.,}$may be equal to zero. The distance $I$, can be calculated as folloms:

$$
1,=(1-m)(P+1)+a t_{1}^{2} / 2+1, t_{1}
$$

\subsection{Profile of Required PSD}

In order to study the safety of the passing manocuvec comprehensvely. the regunred sught distance profile should be determined. The portion of the protsle prion la the culleal

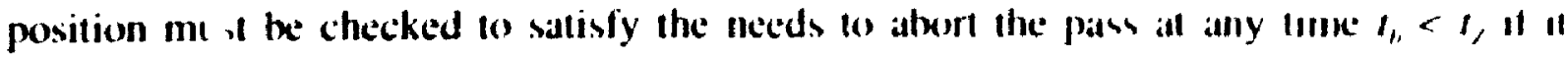

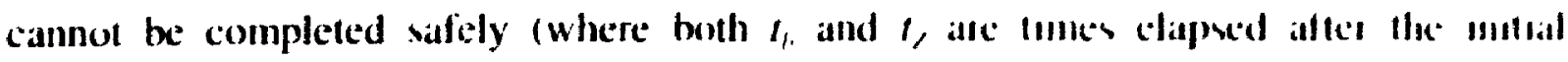
pereeption-reaction time). Although the passng vehicle call complele the pass sately ance

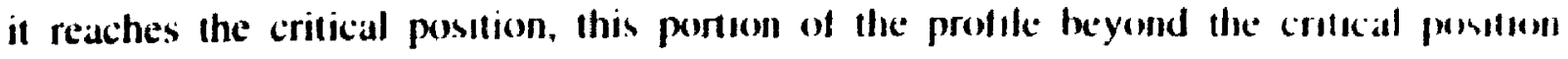
should be checked to ensure that the opposing vethele is vishble to the driver of the 
passing vehicle at any time $t_{1}>t_{1}$. Therefore, this section presents the derivation of the required sight distance, $S$, at any time $t_{1 ;}$. The derivation of the required sight distance depends on whether $t_{t}$, is less or greater than $t_{f}$. where $t$ is the time required for the passing vehicle to complete its acceleration ard reach the full design speed, $v$, and equals m/a. Generally, three cases can be encountered.

Case 1: $\left(t_{b}+P\right) \leq t_{f}$. In this case, in aborted passes, the passing vehicle is assumed to keep accelerating during the entire perception-reaction time, $P$. The speed of the passing vehicle at the beginning and end of the perception-reaction time, $v_{1}$ and $v_{2}$, will be ( 1 $\left.m+a t_{1}\right)$ and $\left(1-m+a t_{1}+a P\right)$, respectively. As shown in Figure 3.6a, the distance between the front bumpers of the passing and the impeding vehicles. $\Delta$. can be calculated as follows:

$$
(1 \cdot-m) t_{1}+a t_{1}^{\prime} / 2-\Delta=(1-m) h+L_{1}+(1-m) t_{1}
$$

or

$$
\Delta=-L_{l}-(v-m) h+a t_{n}^{2} / 2
$$

If $t_{1}<1$, , the pass should be aborted. Referring to Equation 3.3 , the values of $\Delta_{1} .1^{\prime}$, and $1_{2}$ can be replaced by $\Delta . v_{2}$, and $\left(v_{1} l+a P^{2} / 2\right)$, respectively. Then, the time $t_{2}$ required (1) abort the pass can be calculated as follows:

$$
(1-m)\left(P+t_{2}\right)=L_{1}+\left(j_{2}+v_{2} t_{2}-d t_{2}^{2} / 2+v_{1} P+a P^{2} / 2+\Delta\right.
$$




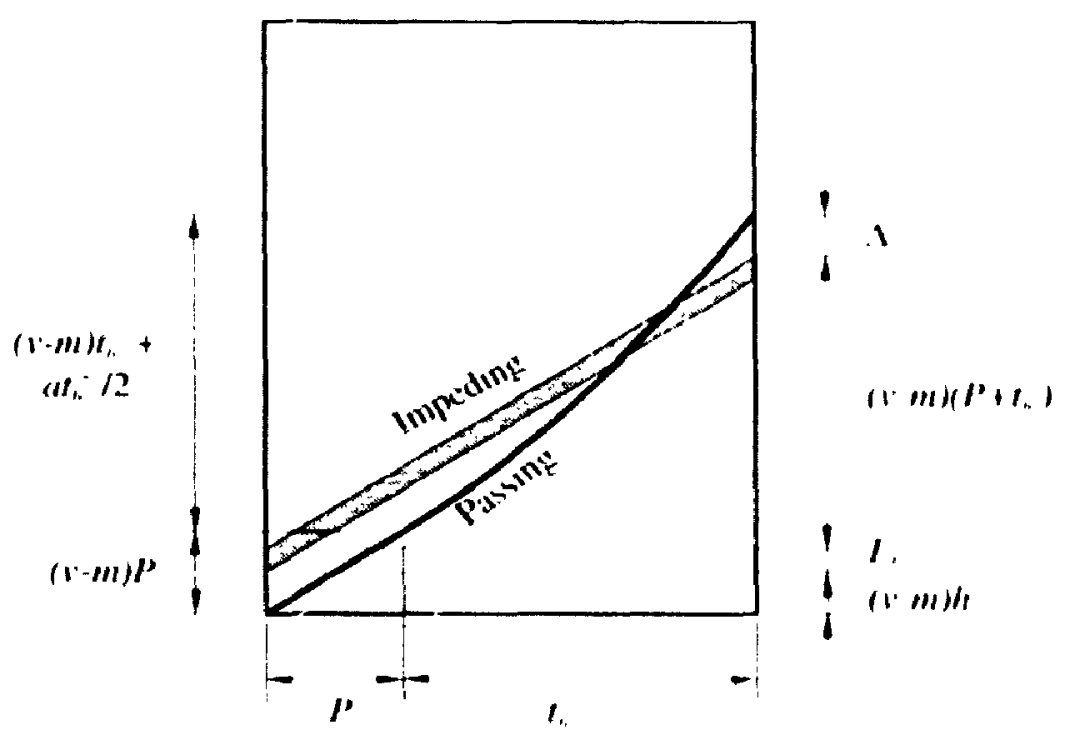

(a) $t, \leqslant t$,

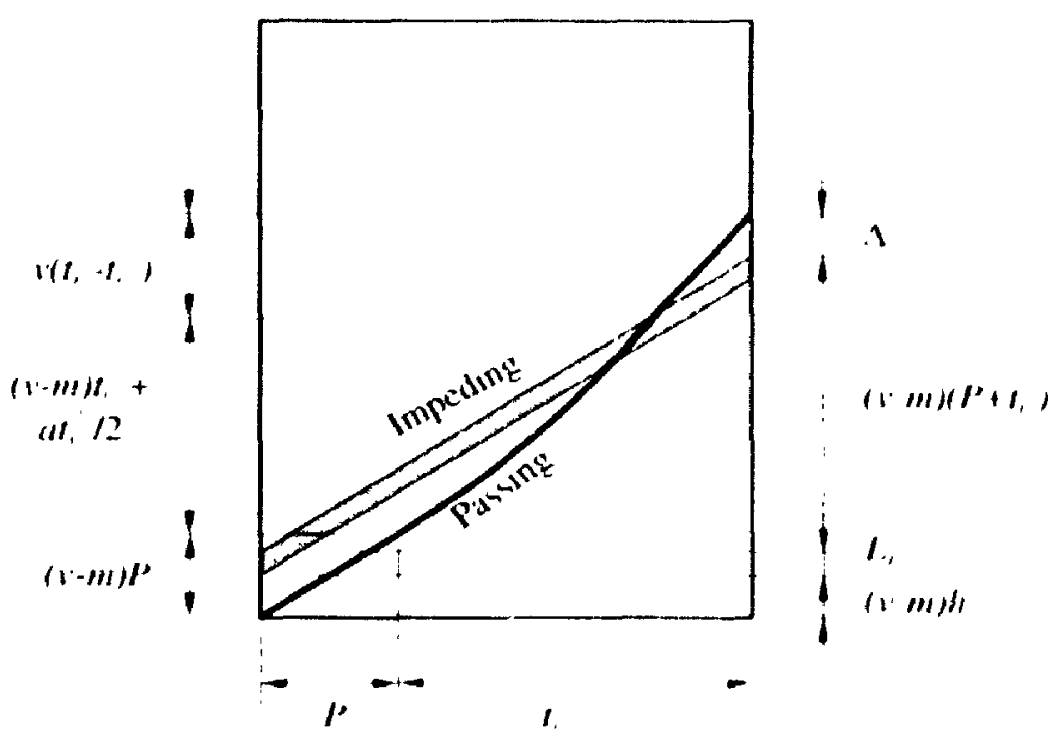

(b) $1,>1$,

FIGLRE 3.6: Relative Positions of the Passing and Impeding Vehicles up to Time $t_{t}$ after Pass Initiation. 


$$
d t_{2}^{2} / 2+\left|d h_{1}-a\left(t_{b}+P\right)\right| t_{2}+\left[(v-m)\left(h-h_{l}\right)-\frac{a}{2}\left(t_{b}+P\right)\left(t_{b}+P+2 h_{1}\right)\right]=0
$$

Then, $s$ can be formulated as:

$$
S=\left(v_{1} P+a P^{2} / 2\right)+\left(v_{2} t_{2}-d t_{2}^{2} / 2\right)+\left(v+v_{2}-d t_{2}\right) h_{0}+v\left(P+t_{2}\right)
$$

On the other hand, if $t_{b}>t_{\partial}$, the pass should be completed, and the time $t_{1}$ required to complete the pass can be calculated by substituting for $\Delta_{1}$ and $x_{1}$ in Equation 3.1 with $\Delta$, and $\left(v_{1} t_{u}+a t_{a}^{2} / 2\right)$, respective:, as follows:

$$
\Delta+\left(v_{1} t_{a}+a t_{a}^{2} / 2\right)+v t_{1}=(v-m)\left(t_{d}+t_{1}\right)+(v-m) h_{1}+L_{1}
$$

or

$$
t_{1}=\frac{L_{t}+L_{p}+(v-m)\left(h+h_{t}\right)-\frac{a}{2}\left(t_{t}+t_{b}\right)^{2}}{m}
$$

Then, $S$ can be formulated as:

$$
S=\left(v_{1} t_{d}+a t_{t}^{2} / 2\right)+v t_{1}+v\left(t_{t}+t_{1}\right)+2 v h_{0}
$$

Case 2: $t_{b}<t_{f}$ and $\left(t_{b}+P\right)>t_{f}$. In this case, in aborted passes, the passing vehicle is assumed to keep accelerating during a portion $P_{1}$ of the perception-reaction time and then, maintain its constant speed. 1 , during the remaining portion $P_{2}$, where $P_{1}=m / a-t_{t}$, and $P_{:}=P-P_{1}$. The speed of the passing vehicle at the beginning of the perception-reaction timc, $v_{1}$, will be $\left(1-m+a t_{n}\right)$. The distance $\Delta$ in this case can be calculated as in Case 1 . 
Then, if $t_{h}<t_{\mu}$, the pass should be aborted. and the time $t_{z}$ can to callculated by substituting for $\Delta_{1}, v_{1}$, and $x_{2}$ in Equation 3.3 with $\Delta, r$ and $\left(v_{1} P_{1}+a P_{1} y_{2}+1 P_{2}\right)$. respectively, as follows:

$$
(v-m)\left(P+t_{2}\right)=L_{1}+G_{2}+v t_{2}-d t_{2}^{2} / 2+v P_{2}+v_{1} P_{1}+a P_{1}^{\prime} / 2+\Delta
$$

or

$$
d t_{2}^{2} / 2+[d h-m] t_{2}+\left[\frac{m^{2}}{2 a}-m\left(h+P+t_{h^{\prime}}\right)+v\left(h-h_{1}\right)\right]=0
$$

Then, $S$ can be formulated as:

$$
S=\left(v_{1} P_{1}+a P_{1}^{2} / 2\right)+v P_{2}+\left(v t_{2}-d / t_{2}^{\prime} / 2\right)+\left(2 v-d / t_{2}\right) h_{1}+v\left(t^{\prime}+t_{2}\right)
$$

If $t_{l}>t_{2}$, the pass should be completed, and $t_{1}$ and $S$ can be calculatted as in ("anse 1 .

Case 3: $t_{b} \geq t_{f}$. As shown in Figure 3.6b, the distance $\Delta$ can be calculalled as follows:

$$
(v-m) t_{1}+a t_{,}^{2} / 2+v\left(t_{b}-t_{f}\right)-\Delta=(v-m) h+t_{i}+(v-m) t_{b}
$$

or

$$
\Delta=-L_{1}-1 \cdot h-\frac{m^{2}}{2 a}+m\left(l_{1}+h\right)
$$

If $t_{1}<t_{Z}$, the time required to abort the pass, $t_{i}$. can be calculated by subsituting for $\Lambda_{,}$, $v_{d}$, and $x_{2}$ in Equation 3.3 with $\Delta . v$, and $v$, respectively, as follows:

$$
(v-m)\left(d^{D}+t_{2}\right)=L_{1}+G_{2}+v t_{2}-d t_{2}^{2} / 2+v P+\Delta
$$




$$
d t_{2}^{2} / 2+\left|d h_{1}-m\right| t_{2}+\left[v\left(h-h_{1}\right)+\frac{m^{2}}{2 a}-m\left(h+P+t_{b}\right)\right]=0
$$

Because the speed of the passing vehicle at the beginning of the deceleration is $v$, as in Case 2, the formulas of $t_{2}$ in the two cases are similar. However, the resulting $t_{2}$ wiil not be the same due to the change in the value of $t_{b}$ in the two formulas.

Then, $S$ can be formulated as:

$$
S-v P+\left(v t_{2}-d t_{2}^{2} / 2\right)+\left(2 v-d t_{2}\right) h_{O}+v\left(P+t_{2}\right)
$$

If $t_{l,}>t_{\ell,}$, both $t_{a}$ and $x_{1}$ in Equation 3.1 will vanish, and substituting for $\Delta_{t}$ with $\Delta$, the time $t_{1}$ required to complete the pass can be calculated as follows:

$$
\Delta+v t_{1}-(v-m) t_{1}+(v-m) h_{1}+L_{p}
$$

or

$$
t_{1}-\frac{L_{1}+L_{p}+(v-m)\left(h+h_{l}\right)}{m}+\frac{m}{2 a}-t_{l}
$$

Then, the required sight distance can be calculated as follows:

$$
S-2 v\left(t_{1}+h_{0}\right)
$$

\subsection{Model Simplification}

The model derived above can be simplified by assuming that, by reaching the critical position. the passing vehicle has completed its acceleration to the design speed, $v$. In this caste. the time-space diagram for the completed and aborted pass can be simplified as shown in ligure 3.7. Foll, wing the same technique, the model can be derived as follows: 


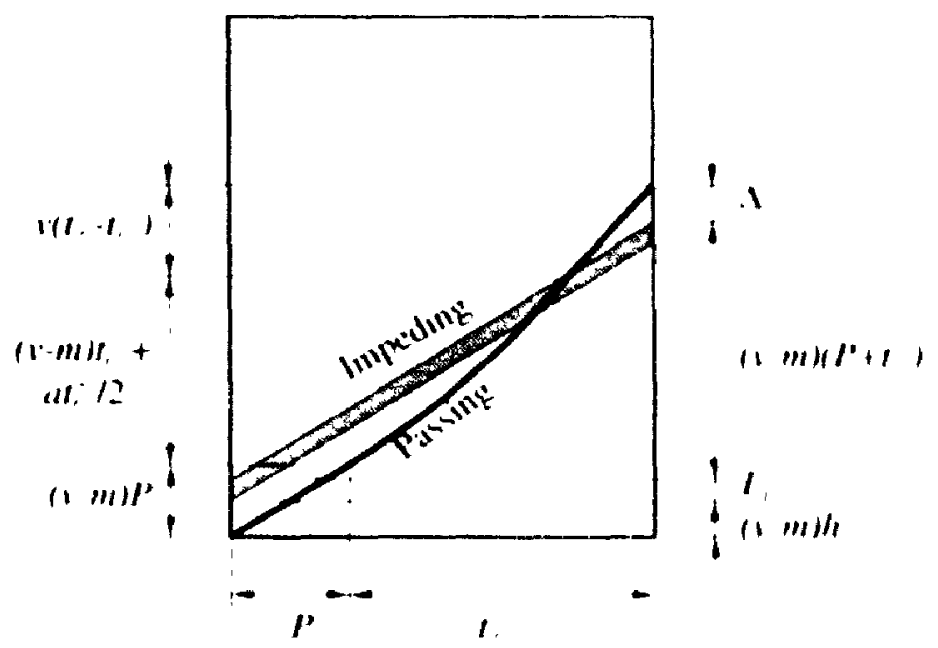

(a) Before the criticall Position

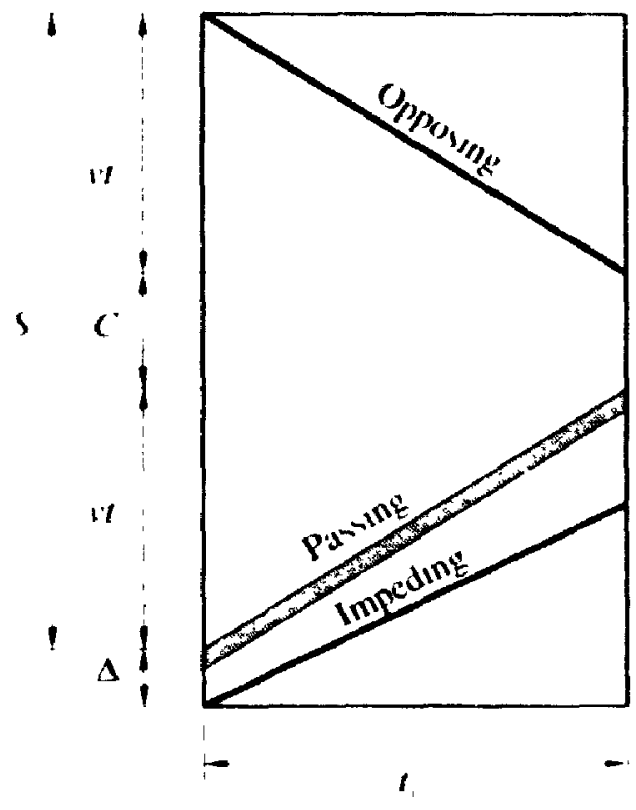

Completed Piss

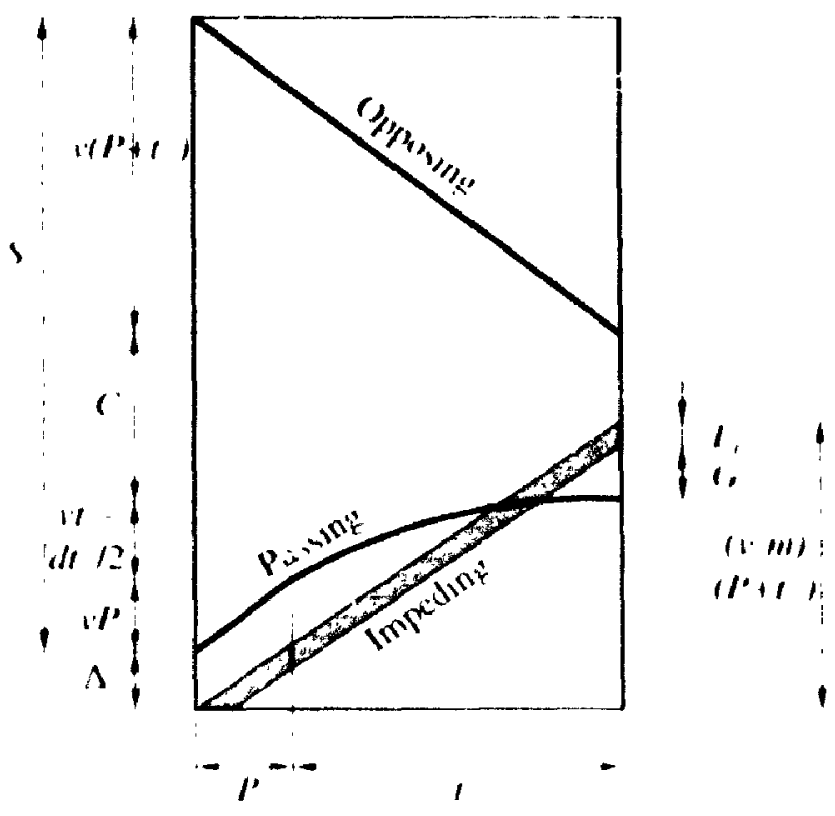

Andued liss

(b) Beyond the C ritical Poutlon.

FIGURE 3.7: Time-Space Diagrams for the Simplificed Model. 
I.or a completed pass.

$$
\Delta_{1}+v t_{1}-t_{p}+G_{1}+(v-m) t_{1}
$$

or

$$
\Delta,-L_{p}+G_{1}-m t_{1}
$$

Similarly, for an aborted pass.

$$
\Delta_{1}+v P+v t_{2}-\frac{d t_{2}^{2}}{2}=(v-m)\left(P+t_{2}\right)-L_{1}-G_{2}
$$

or

$$
\Delta_{1}-\frac{d t_{2}^{2}}{2}-m\left(P+t_{2}\right)-L_{1}-G_{2}
$$

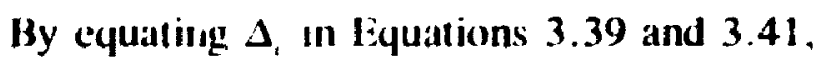

$$
t_{1}-P+t_{2}-\frac{d t_{2}^{2}}{2 m}+\frac{L_{P}+L_{1}+G_{1}+G_{2}}{m}
$$

By equating $S$, for the completed and aborted passes,

$$
2 v t_{1}+C_{1}-v P+v t_{2}-\frac{d t_{2}^{2}}{2}+C_{2}+v\left(t_{2}+P\right)
$$

or

$$
t_{1}-P+t_{2}-\frac{d t_{2}^{2}}{4 v}-\frac{C_{1}-C_{2}}{2 v}
$$

From liquations 3.42 and 3.44 . 


$$
t_{2}^{2}\left[\frac{d(2 r-m)}{4 \cdot m}\right]=\frac{L_{1}+L_{1}+C_{1}+C_{i}}{m}+\frac{C_{1}-C_{2}}{2 v}
$$

Substituting for the values of $G_{1}, G_{2}, C_{1}$, and $C_{2}$ and solving for $t_{:}:$

$$
t_{2}=-\frac{21 \cdot h_{1}-m h_{11}}{21-m}+\sqrt{\left[\frac{21 \cdot h_{1}-m h_{11}}{21-m}\right]^{2} \cdot \frac{4 \cdot \mid h_{1}+l_{1}+(21) m\left(m h_{1} \mid\right.}{d(21-m)}}
$$

Note that the other possible value of $t_{2}$ is megallive and thus matmissible. The'll, llus values of $t_{1}, \Delta_{1}$. and $S_{\text {c }}$ can be formulated as:

$$
\begin{aligned}
& t_{1}=P+t_{2}-\frac{d t_{2}}{4 v}\left(t_{2}+2 h_{0}\right) \\
& \left.\Delta_{c}=L_{p}+(1-m) h_{1}-m t_{1}=\frac{d i}{2}-m(p)+t_{2}\right)-L_{1}-\left(1 \quad d 1,1 h_{i}\right. \\
& S_{c}=2 v\left(t_{1}+h_{0}\right)=2 v^{\prime}\left(P+t_{2}+h_{0}\right)-\frac{d t_{2}^{2}}{2} \cdot d t_{\imath} h_{\prime \prime}
\end{aligned}
$$

If $h_{l}=h_{l}=h$, Equation 3.46 through 3.49 can be wrillen an lollows:

$$
\begin{aligned}
& t_{2}=-h_{1}+\sqrt{h^{2}+\frac{4 v\left[L_{,}+L_{1}+(2 v-m) h\right]}{d(2 v-m)}} \\
& t_{1}=P+t_{2}-\frac{d t_{2}}{4 v}\left[t_{2}+2 h\right]
\end{aligned}
$$

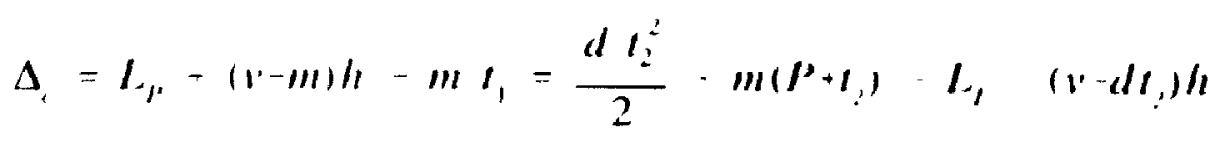




$$
S_{1}-2 v\left(t_{1}+h\right)=2 v\left(P+t_{2}+h\right)-\frac{d t_{2}^{2}}{2}-d t_{2} h
$$

If $\Delta,>0$, as shown previously, the time required to complete the pass in this case, $i_{1}$, can be calculated by substituting for the value of $\Delta_{c}=0$ in Equation 3.48 as follows:

$$
t_{1}^{*}=\frac{(v-m) h_{1}+L_{r}}{m}
$$

Then, Equation 3.49 can be written as follows:

$$
S_{s}=\left\{\begin{array}{cc}
2 v\left(t_{1}+h_{0}\right) & \Delta_{c} \leq 0 \\
2 v\left(t_{1}^{*}+h_{0}\right) & \Delta_{c}>0
\end{array}\right.
$$

The derivations for $t_{Z}$ and $l_{Z}$ can also be simplified using the same assumption that the passing vehicle has completed the acceleration before reaching the critical position. In this case, the time-space diagram for the pass before the critical position will be as shown in Figure 3.7a, and $t$, and $l$, can he formulated as follows:

$$
\begin{aligned}
& t_{l}=\frac{m}{2 a}+\frac{\Delta_{1} i_{1}+v h}{m}-h \\
& l_{l}-\Delta_{1}+(v-m)\left(P+l_{Y}+h\right)+L_{1}
\end{aligned}
$$

The derivations for the profile of the required sight distance will not be affected by this assumption. However, in this case, Cases 1 and 2 will not be applicable for completed passes. 


\subsection{Selection of Mlodel Parameters}

Differential Speed $(m)$ : In the AASHTO design guide, the differential specd. $m$, is set as a constant value of $15 \mathrm{~km} / \mathrm{h}$ regardless of the design specd. $\mathrm{s}$. (O) the wher hand. based on field studies, speed dependant values of m were assumed by (ilennon ( I)ss) and Harwood and Glennon (1989). These values can be related to the design speed, I. using the following formula:

$$
m=24-V / 10
$$

where $m$ and $V$ are in $\mathrm{km} / \mathrm{h}$.

Deceleration Rate (d): Although the deceleration ratte of the passing vehicke, d, in the presented model is assumed constant, an iterative precedure can he used lo alcount for a speed-dependent deceleration rate as follows:

1. Assume an initial value for the deceleration ralte. $d$.

2. Calculate $t_{2}$ as shown previously.

3. Calculate the final speed of the passing vehicle, $v_{f}$, ats $\left(v_{1}, d t\right)$, or $(1-d t)$ according to the simplified model.

4. Select an appropriate model for the speed-dependent deceleration tate iA ASITIO). 1994: Olson et al, 1984; French. 1982) and delermine line average deceles allon sate corresponding to these initial and final speeds

5. Continue the iterations until the change in the value of d In Iwo stecessive Hetalloms is within the requised accuracy. 
Among the different available models for deceleration, the model presented by Olson et all (1984) for a worn tire to $1.59 \mathrm{~mm}$ (2/32 inches) and operation with steering cortrol should provide a sufficient braking distance for virtually all the vehicles on a highway. Therefore, it is recommended in this research. According to this model, the average deceleration rate from the design speed. 1 , to the final speed, $v$, can be calculated as follows:

$$
d=\frac{v^{2}-v_{1}^{2}}{2\left(B D_{0}-B()_{1}\right)}
$$

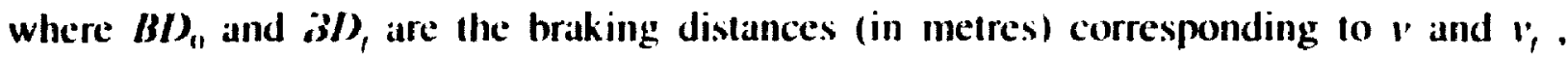
respectively.

Table 3.1 shows the braking-distance data for a passenger car with a worn tire and decelerating with steering control. The braking distance corresponding to any speed can be calculated using Gauss interpolation. However, because these data were developed assuming a locked-wheel condition for speeds lower than $32 \mathrm{~km} / \mathrm{h}$ ( $20 \mathrm{mph}$ ) (Olson et al, 1984), only the braking distance corresponding to higher speeds are to be used in calculating $d$. This can be done by imposing a maximum deceleration rate that corresponds to deceleration from the design speed to $32 \mathrm{~km} / \mathrm{h}$. Finally, to avoid the situation where the passing vehicle decelerates to unreasonably low speed, the final speed at an aborted pass can he set to a minimum value, $1 ;$. This can be done by reducing the value of $d$ to allow deceleration during the entire time interval $t_{z}$ and maintaining the final speed $r$. 
TABLE 3.1: Braking Distance for a Passenger (ar with Tires

Worn to 2/32 inch in Operation with Stecring

Control (Olson et al, 1984$).$

\begin{tabular}{cc}
$\begin{array}{c}\text { Speed } \\
(\mathrm{km} / \mathrm{h})\end{array}$ & $\begin{array}{c}\text { Brahing Distanci } \\
(\mathrm{m})\end{array}$ \\
\hline 32 & 13.72 \\
42 & 3.3 .53 \\
64 & 0.5 .84 \\
80 & 115.82 \\
97 & 188.67 \\
113 & 287.4 .3 \\
129 & 416.05 \\
\hline
\end{tabular}

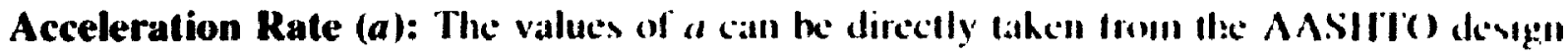
guide (Table 3.2). Another model was developed by (ilaule el al (los(1) and call also lxe used. Accarding to this model, the acceleration rate, a $\left(\mathrm{m} / \mathrm{s}^{\prime}\right)$, call Ix calculatled att all arbitrary speed, $v_{\triangleleft}(\mathrm{m} / \mathrm{s})$, as follows:

$$
\begin{aligned}
& a_{0}=1.14\left[\frac{2-e^{1+24 k}}{1-e^{1+55 k 1}}\right] \\
& v_{n}=\frac{a_{10}}{0.085} \\
& a=a_{0}\left(1-v_{13} / b_{m}\right)
\end{aligned}
$$

where

$$
K=\text { mass-to-power ratio }(\mathrm{kg} / \mathrm{W}) \text {. }
$$


$a_{11}=$ maximum accelc stion $\left(\mathrm{m} / \mathrm{s}^{2}\right)$. and

$v_{m}=$ naximum speed $(\mathrm{m} / \mathrm{s})$.

However, because the speed increases continuously upon acceleration, an average sped (1-m/2) can be used to calculate the acceleration rate. Also, a low power-to-mass ratio of $40 \mathrm{~W} / \mathrm{kg}$ which represents a relatively poor performance is recommended for paswenger cars to account for most of the vehicles. However, because such a vehicle cannot operate on high speeds (the maximum speed $121.9 \mathrm{~km} / \mathrm{h}$ ) and $\mathrm{becase}$ tiais model Iends to underestimate acceleralion capability at high speeds (Glauz et al. 1980). the acecleration rate corresponding to a tiir)-km/h design speed can be assumed to be the same for higher speceds.

TABLE 3.2: Initial Acceleration in Passing Manoeuvres (AASHTO, 1994).

\begin{tabular}{cc}
$\begin{array}{c}\text { Speed ciroup ot } \\
\text { Passing Velicle } \\
(\mathrm{km} / \mathrm{h})\end{array}$ & $\begin{array}{c}\text { Average Acceleratio I } \\
(\mathrm{km} / \mathrm{h} / \mathrm{sec})\end{array}$ \\
\hline $50-6.5$ & 2.25 \\
$66-80$ & 2.30 \\
$81-95$ & 2.37 \\
$96-110$ & 2.41 \\
\hline
\end{tabular}

Vehicle length: The vehicle length is extremely variable. However, a design length of 5 ill can be assumed for passenger cars. On the other hand. the length of a devign truck 
can be taken as $25 \mathrm{~m}$ which is the matimum truch kength on C:Mbalian mids aliend et al. 19911 .

\subsection{Model Validation}

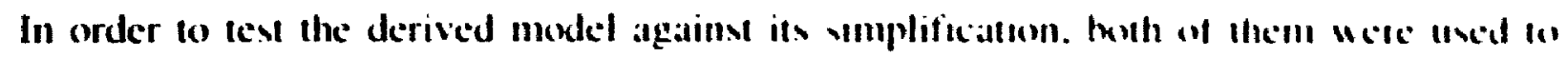

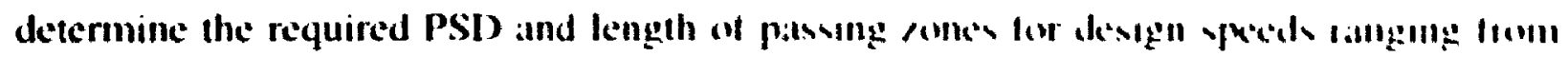

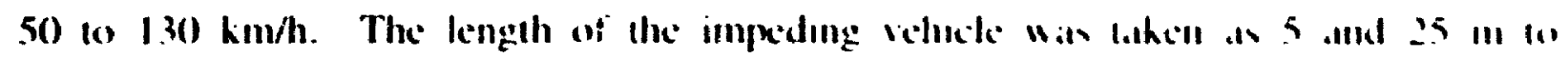

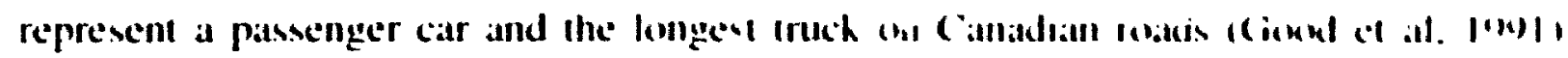

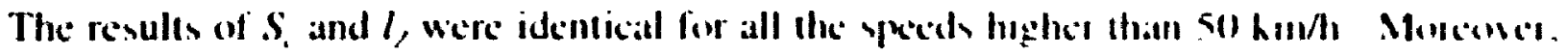

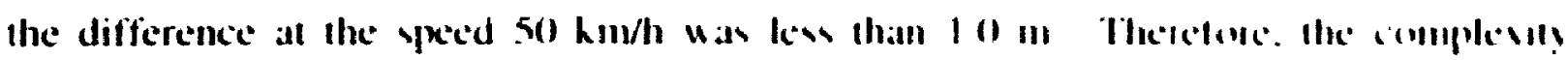

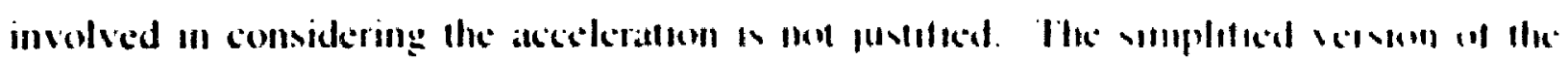

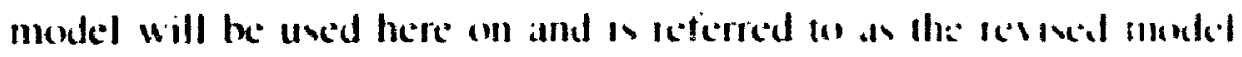

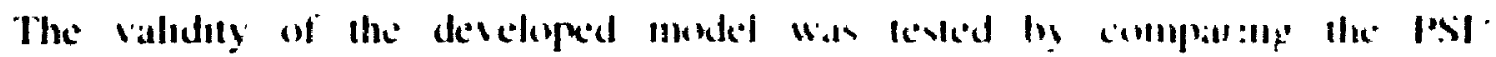

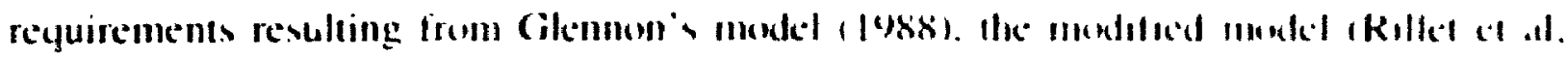

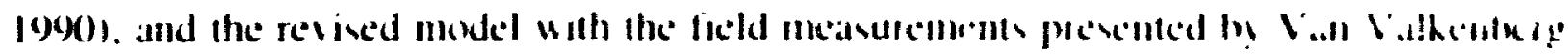

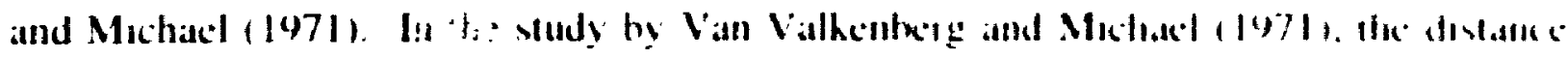

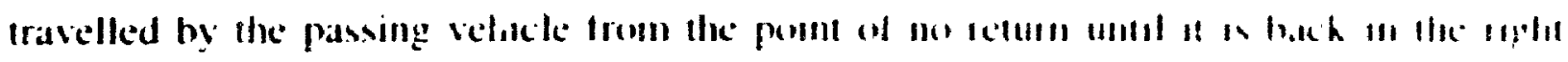

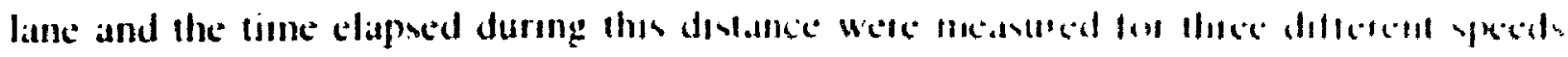

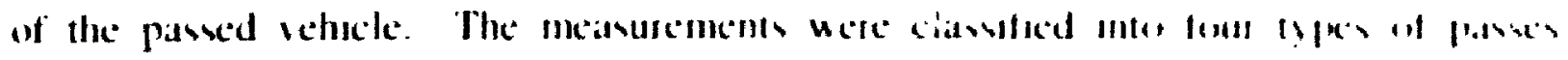

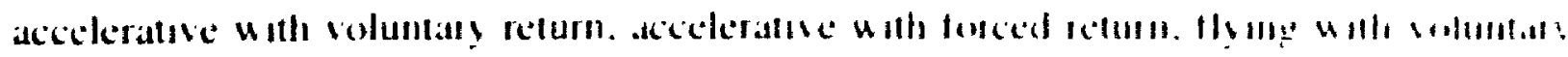

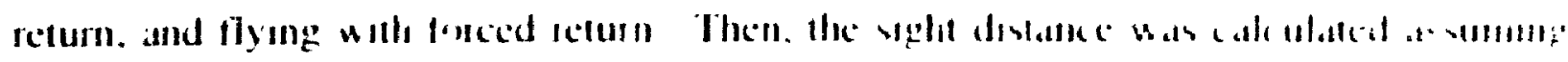




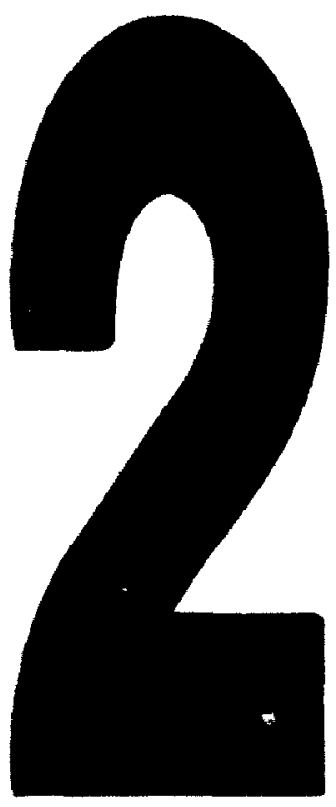

PM-1 3"'" " PHOTOGRAPHIC MICAOCOPY TARGET MES 1010: ANSI/ISO "2 EOUIVALENT

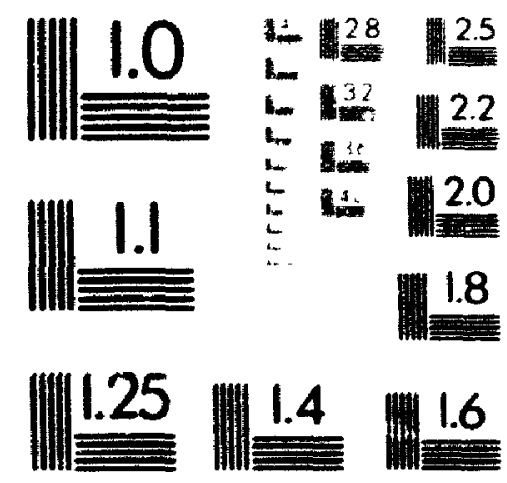

PRECISIONEM RESOLUTION TARGETS 
an opposing vehicle travelling w ith a speed greater than the alvage speed by $11.2 \mathrm{~km} / \mathrm{h}$ $(7 \mathrm{mph})$ and a head-on clearance of $0.1 \mathrm{~m}$ (20) fil) at the end of the pass

The three models were used to determine the requered PSI ) for tiath speced of the passed vehicle according to the following assumptions:

1. Only the accelerative passes were ennsidered hecaltes all the models assume that the passing vehicle is trailing the passed vehicle at the begming of the manceure.

2. The deceleration rate was taken so as to simulate the operation wath sfermes comtrol for a passenger car with tires worn to 2/32 inches modeled by ()torn et al (1984t. These rates were $2.14,1.88$, and $1.55 \mathrm{~m} / \mathrm{s}^{2}$ for the 4 perds 77.25 , 90.12 , and 111.01 $\mathrm{km} / \mathrm{l}$, respectively.

3. The speed differential, $m$, was calculated using equation 3.58 . The values of $m$ were 16.3. 15.0 , and $12.9 \mathrm{~km} / \mathrm{h}$ for the speeds 77.25 .90 .12 , and $111.04 \mathrm{~km} / \mathrm{h}$. respecturely.

4. The clearance between the passing and opposing velicle alt the end of the pass was taken as $21 \%$ instead of the $6.1 \mathrm{~m}(20 \mathrm{ft})$ assumed by Van Valkenherg and Michatel.

5. The acceleration rate used in the modified model (Rillet et al, l90(0) was assumed according to the values given by AASHTO (1094) for the initial acceleratlon in the passing manoeuvre (Table 3.2 ).

As shown in Figure 3.8, the PSD requirements resultung from (ilemmon's nuxlel are closer to the forced return performance indicating uncomfortable or unsale manoeuvring. On the other hand, the PSD requirements iesulting from Rillet's modified model are much longer than those required for safe and comfontable manisuvring indicating that the model is too conservative. These conservative results are not fustified 


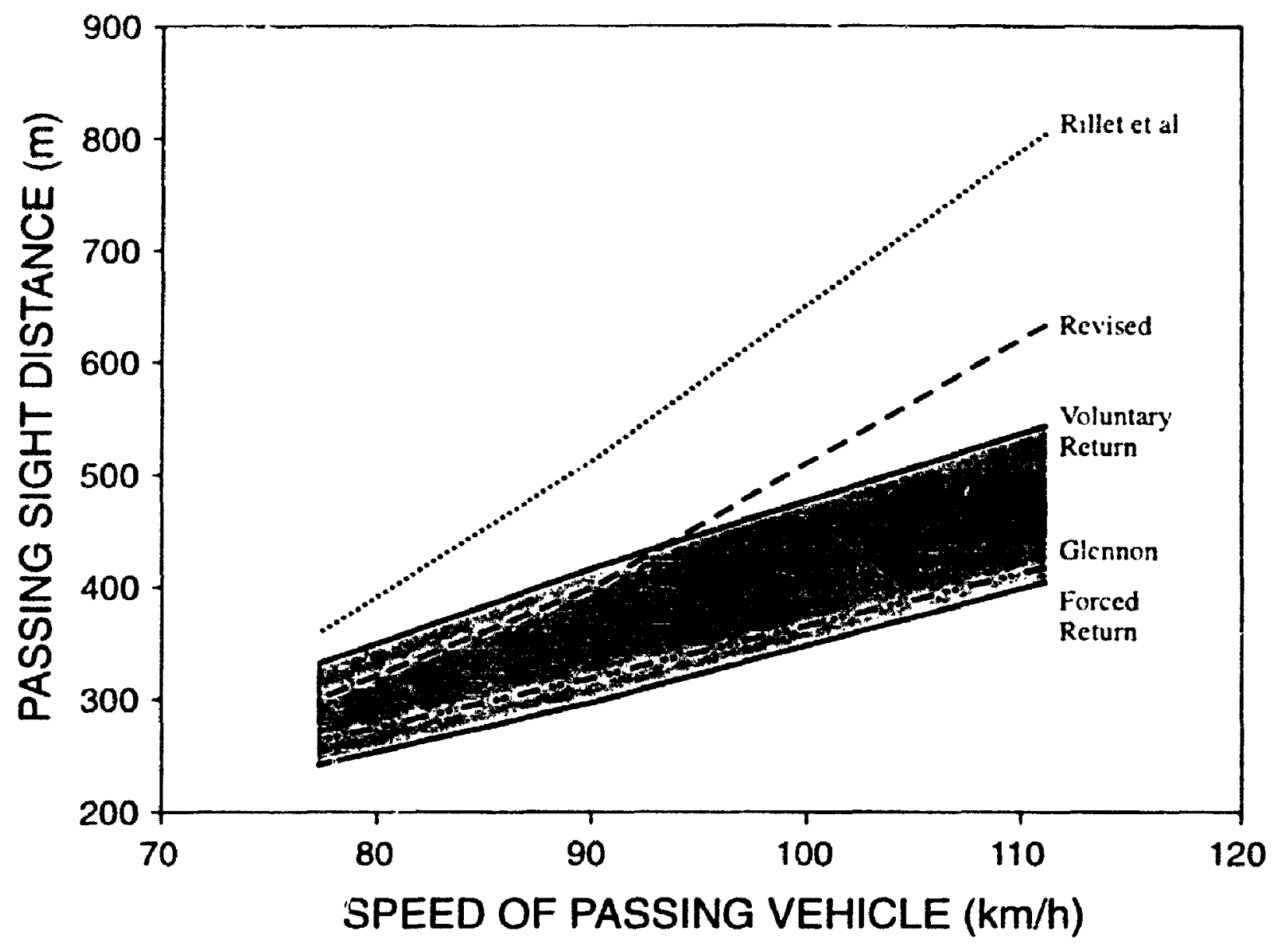

FIGURE 3.8: Validation of the Model for Required PSD.

by ficld observations. The revised model, however, provides PSD requirements that are very close to the voluntary return field data, and therefore ensures safe and comfortable passing manocuvres. Interestingly, the margin of safety and comfort of PSD requirements produced by the revised model increases as the design speed increases, and thus, the degree of potential hazards due to human errors or shifting from the model's assumptions increases. An example of shifting from the model's assumptions is a higher perception- 
reaction time due to any type of impairments such as fatigue. This mallgin of salcty would overcome these hazards.

\subsection{Practical Considerations}

\subsubsection{Design Values for Required PSD}

The revised model presented here was used to develop design valtes for the requised l'SI) on two-lane highways, as shown in Figure 3.9. The PSD requirements when the impeding vehicle is a truck are longer than those when the impeding velicke is a passenger car for speeds up to $110 \mathrm{~km} / \mathrm{h}$. For higher specds, there is $\mathrm{mo}$ difference because the critical position was set as $\Delta=0$ instead of $\Delta_{c}$ which was positive at higher

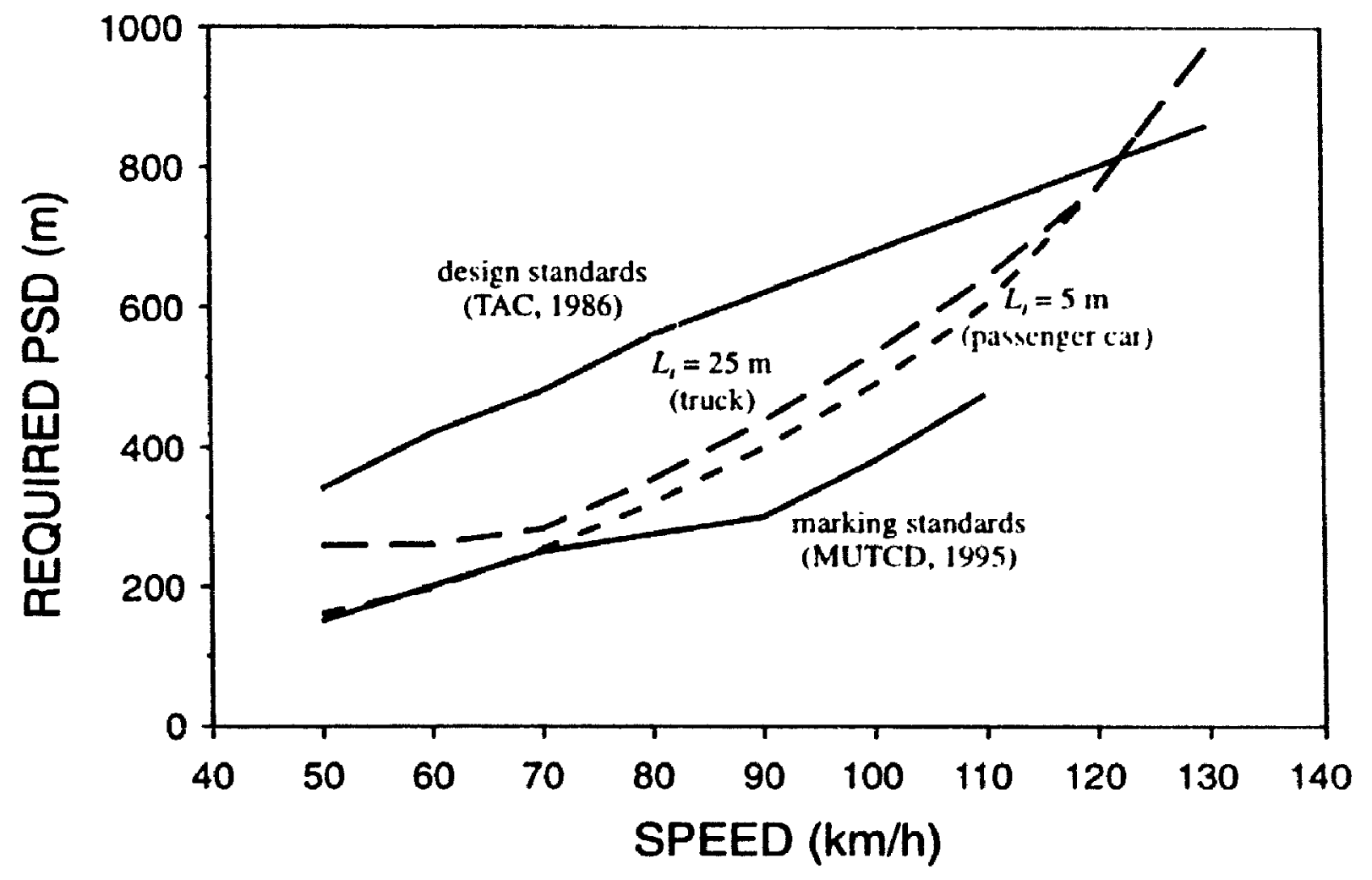

FIGURE 3.9: Comparison of Required PSD of the Model and Current Practice. 
speeds. As expected, this process would produce PSD requirements which are independent of the characteristics of the impedirg vehicle. On the other hand. since the values recommended in the design and the marking standards are to be used for any highway regardless of the traffic composition. both standards fail to consider the effect of the vehicle length. The results also show, in addition to the great difference between the two standards, that the PSD requirements in neither of them would help achieve safe and economic roads. Although following the design standards would guarantee the safety of the passing manceuvres for all passes up to a $120-\mathrm{km} / \mathrm{h}$ design speed. this safety would te achieved in an expensive way. On the other hand. following the MUTCD standards would jeopardize the passes involving passenger cars when the design speed is higher than $70 \mathrm{~km} / \mathrm{h}$. If the impeding vehicle is a long truck, safety would not be achieved for all speeds. It is clear, therefore, that the MUTCD marking standards need major revisions to account for the traffic composition on any specific highway and to ensure safety and comfort in all passing manoeuvres.

\subsubsection{Design Values for Minimum Length of Passing Zones}

Similar to the required PSD, the marking standards fail to consider the traffic characteristics in setting the minimum length of passing zones. Figure 3.10 shows the minimum length of passing zones developed using the model presented earlier and the values recommended in the MUTCD (1976: 1995). The values recommended in the MUTCD (1995) and used for marking in Ontario are higher than the required length for low speeds, and this difference can be related to practical considerations. Moreover, the 


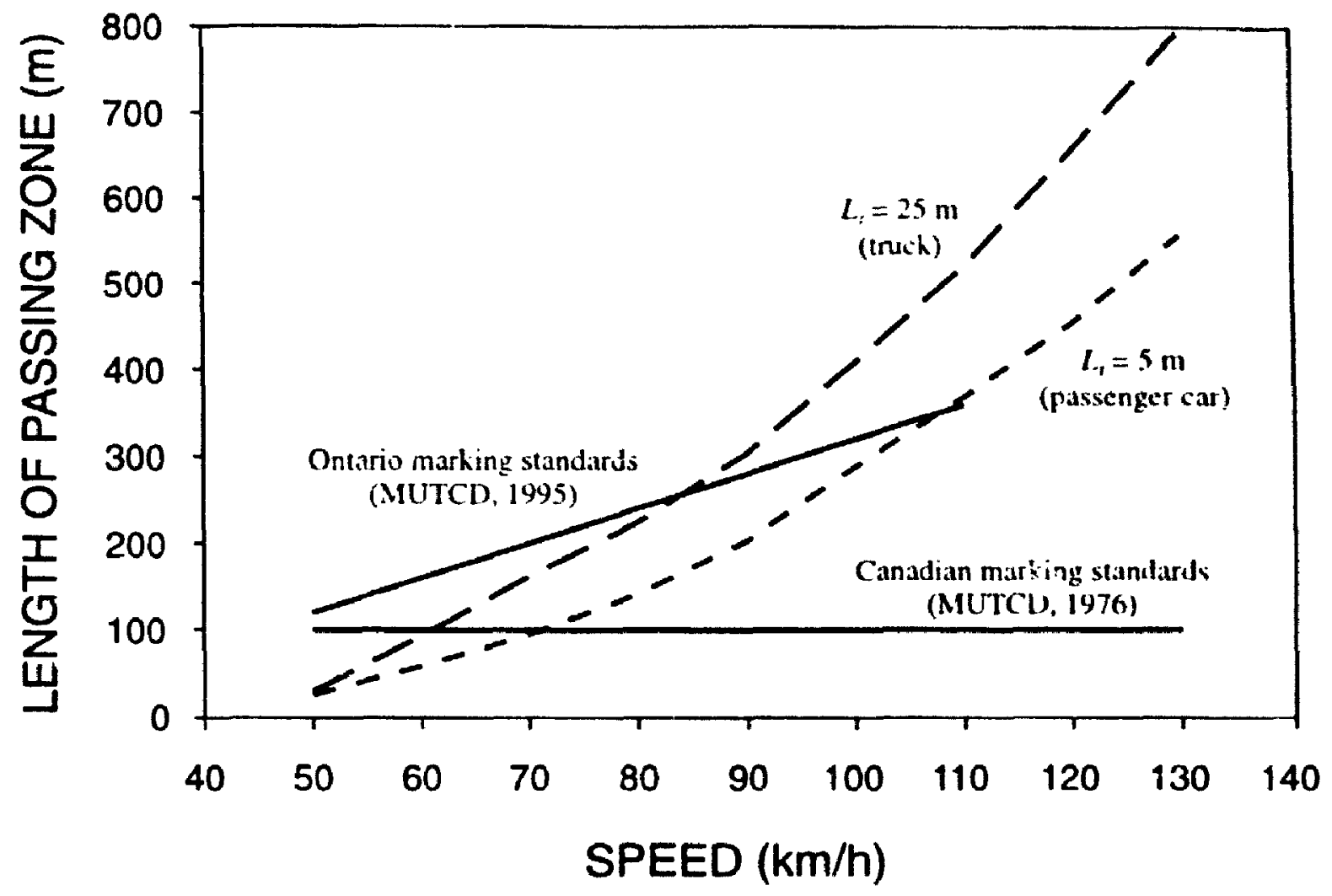

FIGURE 3.10: Comparison of Minimum Length of Passing Zones of the Model and Current Practice.

values are satisfactory for all speeds if the manoeuvre involves passenger cars only. However, if the impeding vehicle is a truck, these values are not sufficient for speeds higher than $90 \mathrm{~km} / \mathrm{h}$. On the other hand, the constant minimum lengths of $100 \mathrm{~m}$ and $122 \mathrm{~m}(400 \mathrm{ft})$ recommended in Canadian and American MUTCD $(1976 ; 1988)$ secm unrealistic.

\subsubsection{Beginning and End of Passing zones}

As mentioned earlier, the profile of required PSD with the evolvement of the pass is useful in checking the safety of the manoeuvre from its beginning to its end. Figure 3.11 shows an example of this profile for a $90 \mathrm{~km} / \mathrm{h}$ design speed. The profile of the required 


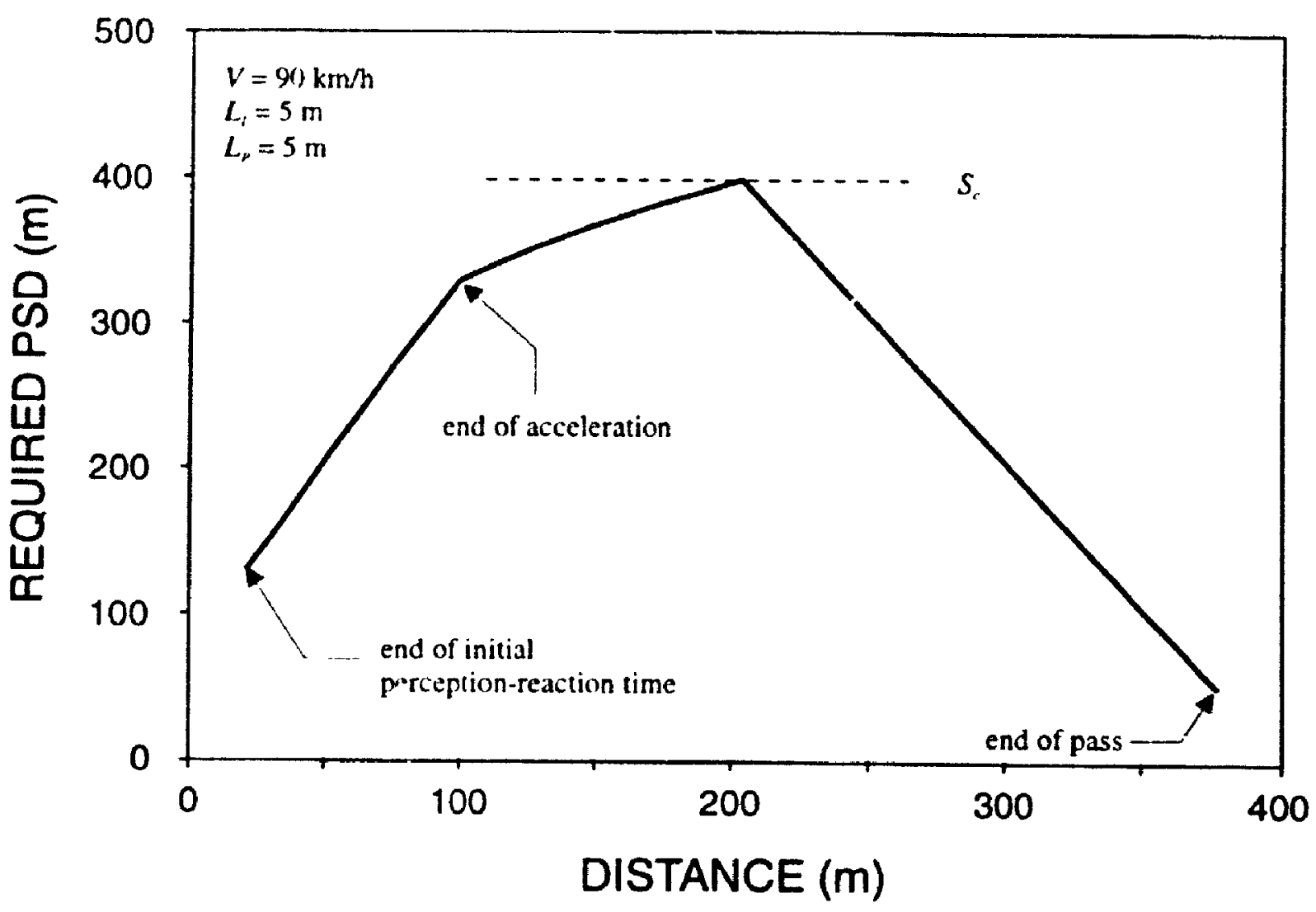

FIGURE 3.11: Example for the Profile of Required PSD.

PSD consists of three distinct zones. The first zone extends from the end of the initial perception-reaction time to the end of the passing vehicle's acceleration time. In this zone, the required PSD is minimum at the end of the ir tial perception-reaction time, and corresponds to aborting the pass. Although the passing vehicle at this point has not begun the acceleration yet, it is assumed that the driver will keep accelerating for a complete perception-reaction time before decelerating again if the pass is to be aborted. The rate of increase of the required PSD in this first zone is relatively high because the speed of the parsing vehicl $s$ increasing.

The second zone extends from the end of acceleration to the critical position. The required PSD in this zone corresponds also to aborting the pass. The speed of the passing 


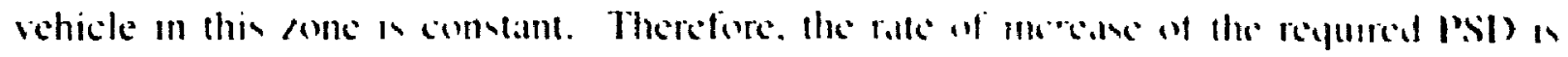

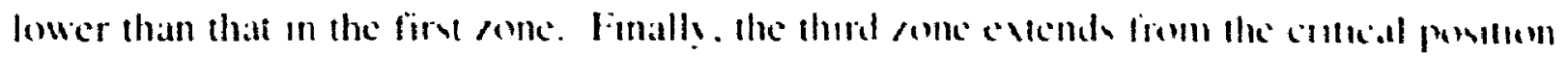

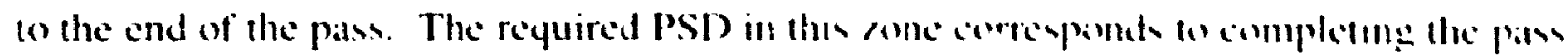

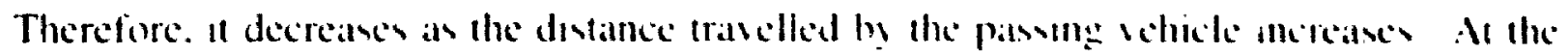

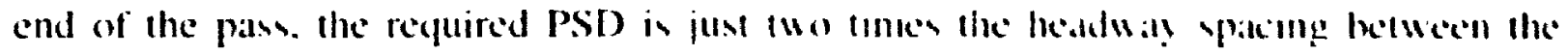
passing and opposing wehicls.

The use of the profile of required PSI) to chech the satety of the pan durmeng the entire inanocuve is shown in Figure 3.12 for different profiles of the at allable PSis

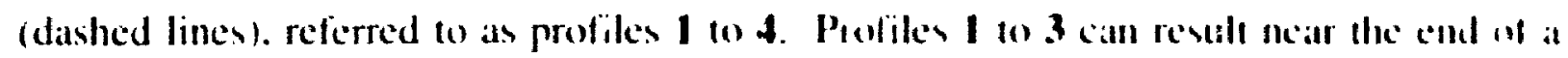
horizontal or a sertical curve where the available sight distance is increasmy. ( In llec other hand. profile 4 can result near the beginning of the curves where the avallahle yght distance is decreasing. For any profile of atailable PSI ) between profiles 1 and 2, a passing zone can begin prior to the point with avalable sight debtalles grealler thall the eritical sight distance. $S_{\text {. }}$. On the other hand, doing the same with profale 3 wrold hude the potential opposing vehicles during a portion of the pass. Therefore, the begimmug of the passing zone must be shifted wh that the requir. d PSD at any tame is kess than the available PSD. Moreover. although the available sght distance in profile 4 is highes than

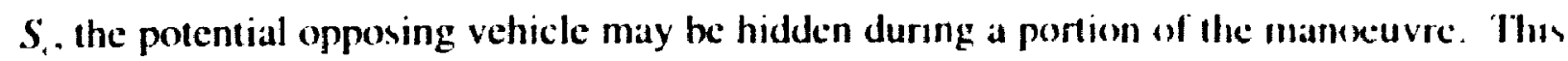
would give a false sense of safety for the passing driver, and therefore may produce lacadon collisions between the passing ans opposing vehicles. Subsequently. such a passllyg manoeuvre should be prohibited by an early marking of a bo-passung anc 


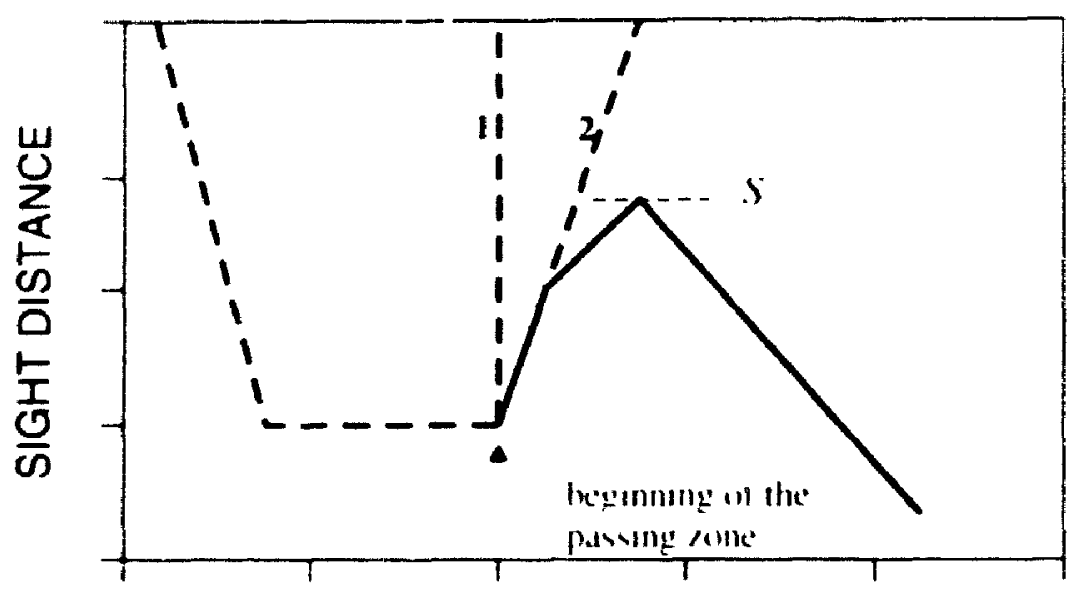

\section{DISTANCE}

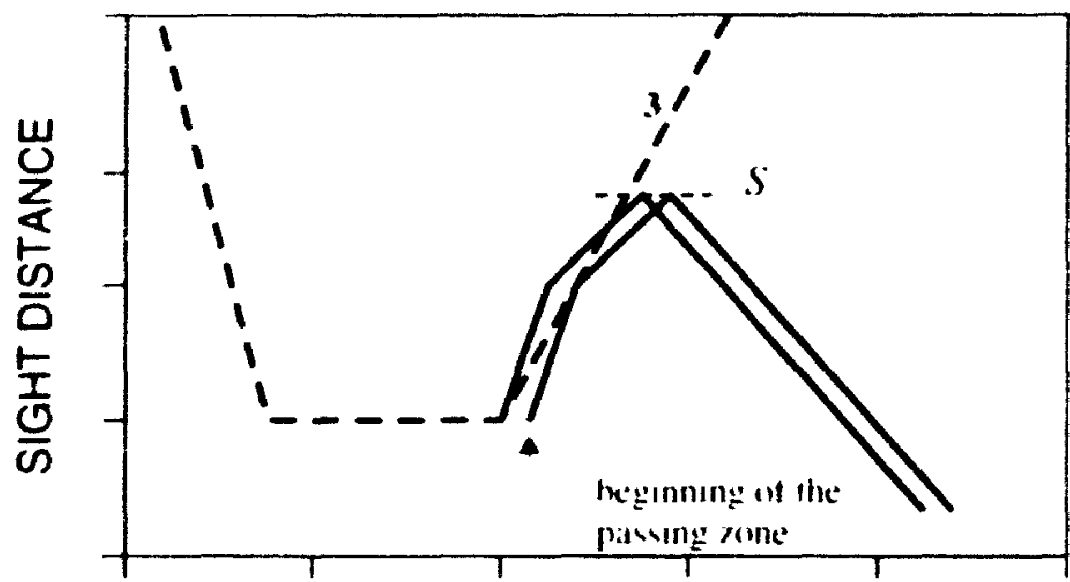

\section{DISTANCE}

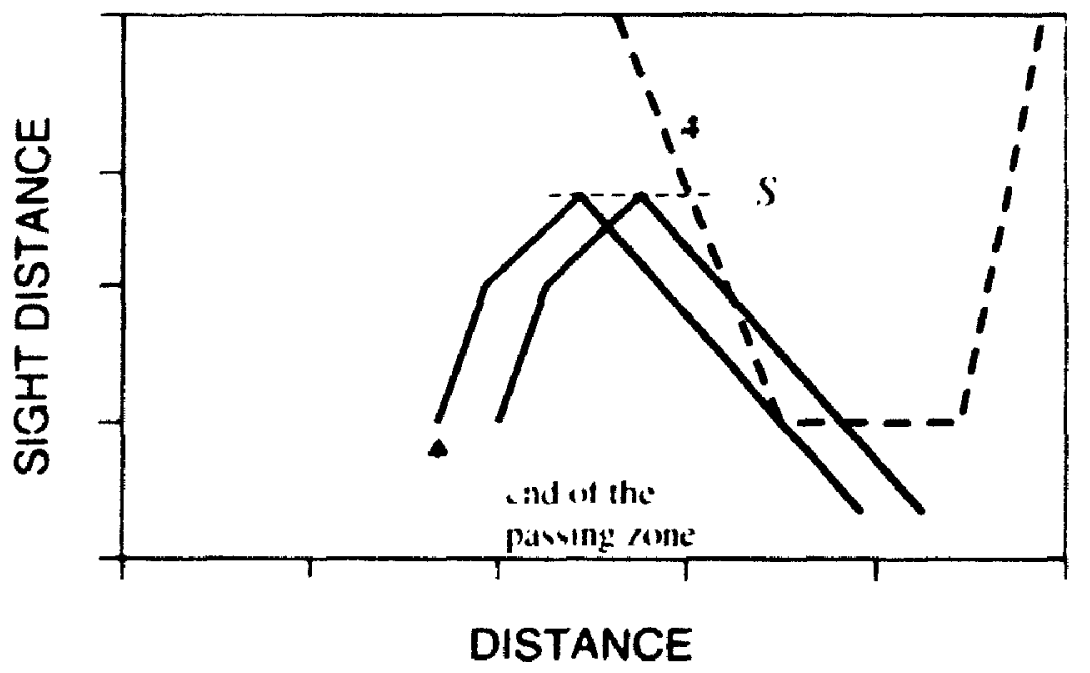

FI(;LRk 3.12: Determination of the Beginning and End of Passing Zones. 


\section{CHAPTER 4}

\section{AVAILABLE SIGHT DISTANCE ON 2-D \\ SEPARATE ALIGNMENTS}

As shown in Chapter 2. current analytical models for available sight distance cannou consider complex 2-D alignments. In addition. in order $t$ evaluate the effect of considering the combined alignment in design. separate horizontal and vertical alignments are studied first. Therefore, this chapter presents general analytical mudels to evaluate sight distance on horizontal alignments for hoth cases of continuous and singli: obstructions (Hassan et al. 1995b: 1995d). Also. models for available sight distance on vertical alignments, where the obstruction may be the ciest curves or the overpasses on sag curves. are presented (Hassan et al. 1995\%; 1995d). The term horizontal alignment is used herein to refer to any combination of the horizontal highway components which are straight segments, circular curves, and clothoid spiral curves. Similarly. the term venical alignment is used to refer to any combination of the highway vertical connponents which are straight segments with constant grade and parabolic sag and crest vertical curves. Firally, computer models are developed to determine the profile of availahle sight distance, and in turn the no-passing zones. on two-lane rural highways

\subsection{Horizontal Alignment with Continuous Obstruction}

Assuming a constant lane width and lateral clearance. the continuous obstruction will be parallel to, and will have the same geometry of, the highway centerline, and the sight 
distance is restricted by having the sight line tangent to the obstruction. The point of langency may he located on a circular curve. spiral curve. or the point of intersection of two successive straight segments without curves. The following sections present general frocedures which can be used to determine the available sight distance regardless of the components of the horizontal alignment. Then. special relationships are presented for the special alignments of simple circular curves and intersecting long tangents. In both of the gencral procedures and special relationships. the lateral clearance hetween the obstruction and the centre of the lane is assumed constant and referred to as $\mathrm{m}$.

In the following sections. the azimuth of a line. defined as the angle between the morth direction and the line, measured clockwise, is used and referred to as $\Phi$. Also, the east and north coordinates of a point are used and referred to as $(x, y)$. respectively. Iowever, since it is the relative positioning of the points to each other. nit the absolute positions. which determines the available sight distance. the coordinates and azimuths can be taken relative to any reference point and direction.

\section{\$.1.I General Procedure: Sight Line Tangent to Circular Curve}

As shown in Figure 4.1. the obstruction restricting the sight line in this case is a curcular curve. In general. the beginning and the end of the sight line may be positioned on any horizontal highway segment (straight. circular curve, or spiral curve). The general procedure. developed in this research, is iterative. where the sight distance is initially assumed as $S$. Then. $S$ is checked and decreased or increased until the sight line becomes tangent to the obstruction. The procedure involves the following steps: 


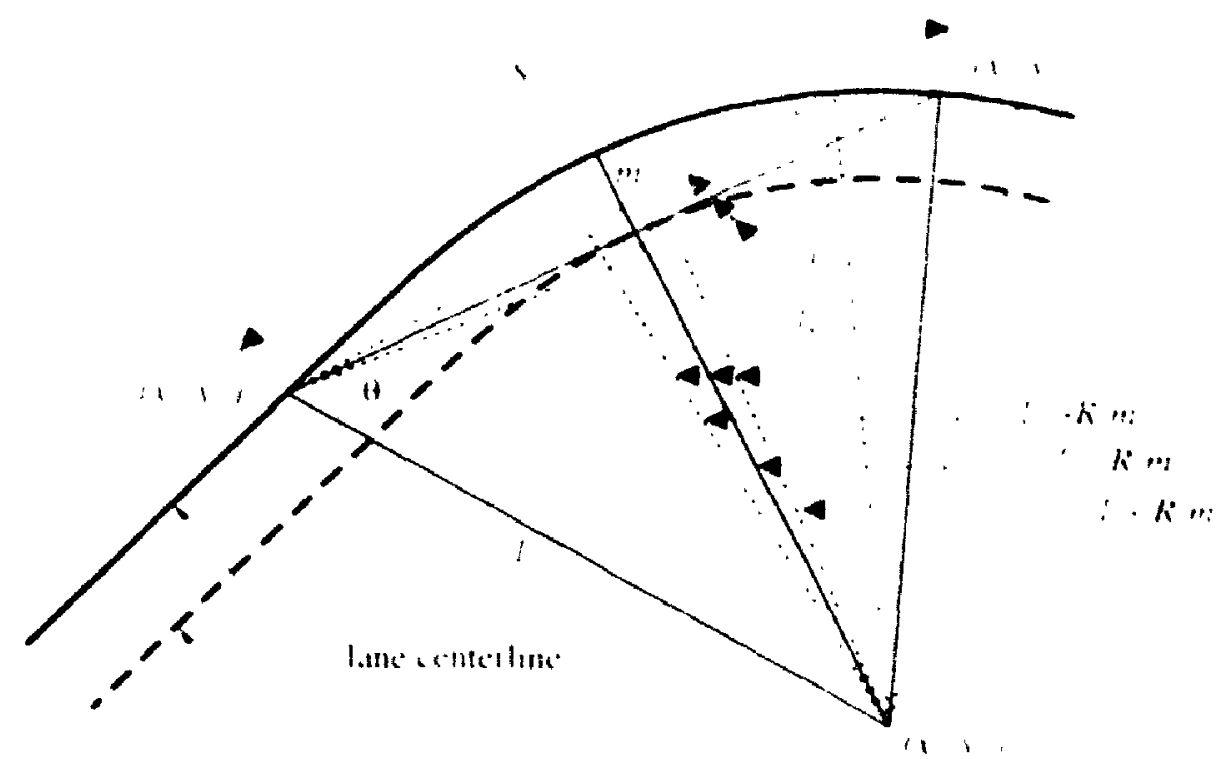

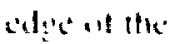

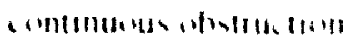

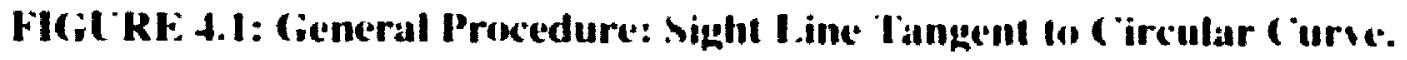
(Continuous (Bbstruction)

1. Determine the coordinates of the beginning of the sight line and the centre of lle curve, $\left(x_{1}, y\right)$ and $(x, y)$, respectively.

2. Calculate the length $l_{1}$ as:

$$
l_{1}=\left\{\left(x_{1}-x_{1}\right)^{2}+\left(y_{1}-y_{1}\right)^{2}\right\}^{2}
$$

3. Determine the coordinates of the end of the sight line. $11,1,1$

4. Calculate $l_{2}$ and $l_{2}$ similar to $l_{1}$.

5. Calculate the angle $\theta$ as:

$$
\theta=\cos ^{-1}\left(\frac{l_{1}^{2}+l_{3}^{2}-l_{2}}{2 l_{1} l_{3}}\right)
$$

6. Calculate the length $l_{4}$ as: 


$$
I_{4}-I_{1} \sin \theta
$$

7 If $l_{4}<\boldsymbol{R}-\boldsymbol{m}, S$ in greater than the actual sight distance. Decrease $S$ and repeat steps $3 \cdot 6$

8. If $I_{4}>\boldsymbol{R}-\boldsymbol{m}, S$ is less than the actual sight distance Increase $S$ and repeat steps 3-6.

9. If $I_{4}=R-m, S$ is equal to the actual sight distance. End of iterations.

Althrogh the lengths $l_{1}, l_{2}$, and $l_{1}$ can he calculated without using the coordinates. a unique sequence of calculations is required for each possible combination of horizontal segments. On the other ha kd, using the coordinates of the points makes the procedure applicable regardless of the positions of the beginning and end of the sight line and also makes it easier for programming.

\subsubsection{General Procedure: Sight Line Tangent to Spiral Cune}

Gemerally. the spiral curve is a curve with varying radius. beginning with a straight segment $(R \rightarrow \infty)$, and as the curve length increases, the corresponding radius decreases. Many mathematical formulas can be used to represent spiral curves and can be found in mathematics textbooks (Drahek. 1969). Among these formulas, Euler's spiral, known as the clothoid spiral. is the most commonly used in road design (AASHTO. 1994; TAC, 1986). Defining $l$ as a segment length of a spiral curve beginning at the point of tangentspiral (TS). $R$ as the corresponding radius. and $\delta$ as the deflection angle of this segment in radian. Euleı's spiral is formulated as follows: 


$$
1=-l \cdot R-\frac{l^{2}}{2 \delta}-R^{2} \cdot 2 \delta
$$

where $A$ is a constant known as the yird parameter

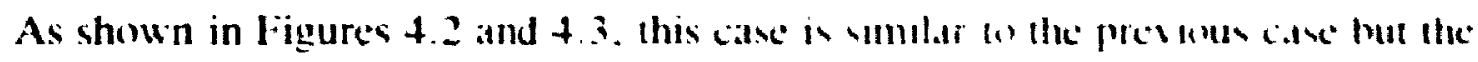
obstruction restrictung the sight line is a spiral curse the hegtmung and the end at the sight line may he positioned on any horizontal highwa! segment etr.tyght. enculal curvi.

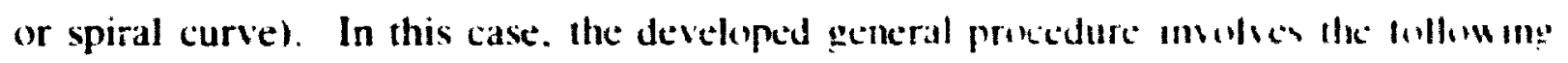
steps:

1. Detennine the coordinates of the heginning of the sight linc, $11,1,1$

2. Determine the coordinates of the end of the sight line. $: 1, \ldots, 1$

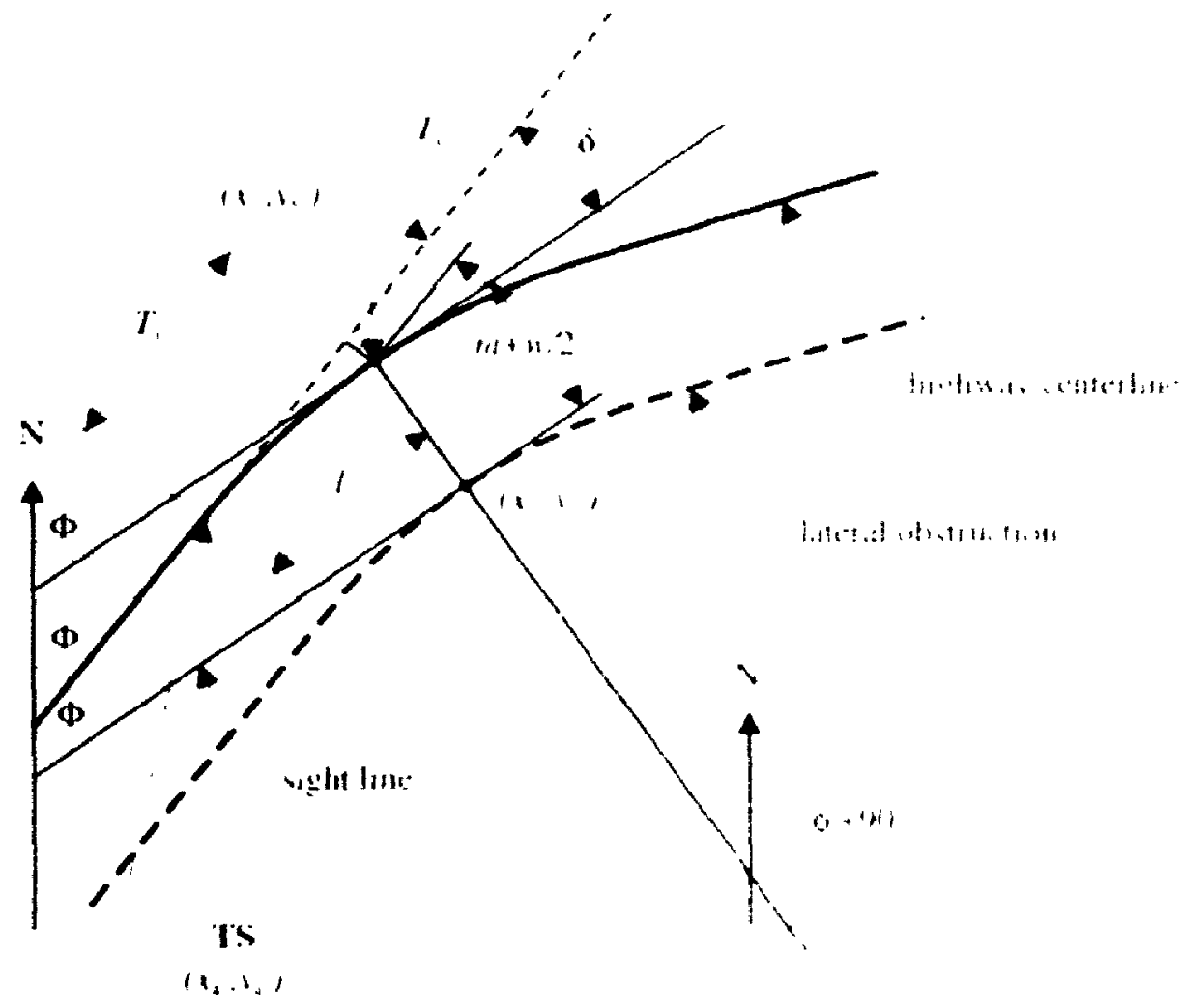

FIGURE 4.2: Determination of Courdinates on tipiral c urves. 


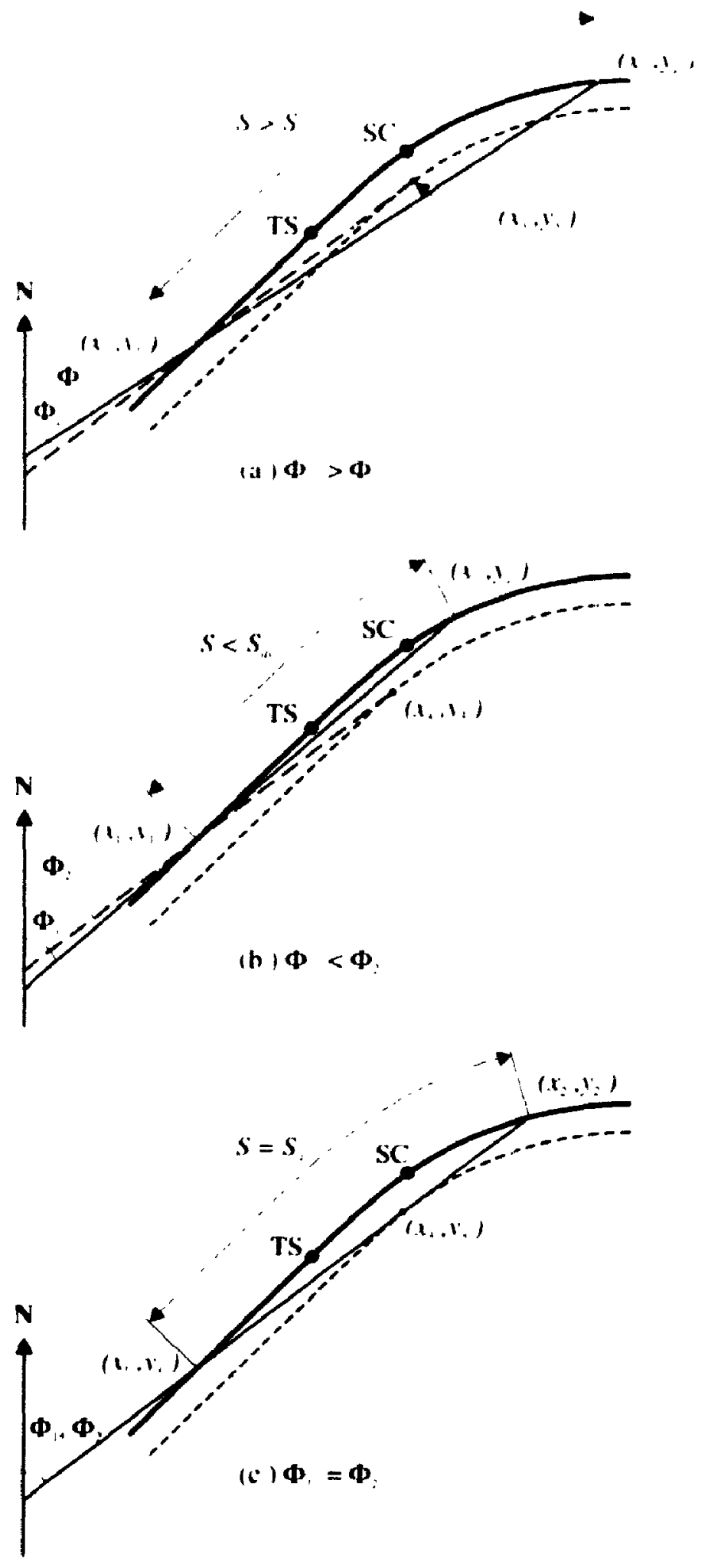

Figure 4.3: General Procedure: Sight Line Tangent to Spiral Curve. (Continuous Obstruction) 
3. Calculate the azimuth of the sight line, $\Phi_{1}$, as:

$$
\Phi_{1}=\tan ^{-1}\left(\frac{x_{2}-x_{1}}{y_{2}-y_{1}}\right)
$$

4. Knowing the azimuth of the tangent to the spiral, $t_{0}$. determine the coordinates of the point of tangency of a line having an azimuth $\Phi_{1}$ and the given obstruction, $\left(x_{3}\right.$. $\left.y_{3}\right)$. For right turn spirals beginning with a straight segment, $\left(x_{1}, y_{3}\right)$ can he determined by considering another point $\left(x_{5}, y_{5}\right)$ defined as the point of tangency of a line having an azimuth $\Phi_{1}$ and the highway centerline. As shown in Figure 4.2 , the coordinates $\left(x_{5}, y_{5}\right)$ can be determined as follows:

$$
\delta=\left(\Phi_{1}-\Phi_{i}\right) \times \frac{\pi}{180^{\circ}}
$$

Using the spiral formula presented in Equation 4.4.

$$
l_{\delta}=A \times \sqrt{2} \bar{\delta}
$$

Fron the general characteristics of Euler's spiral (Drábek. 1969).

$$
\begin{aligned}
& T_{x}=A \sqrt{2} \bar{\delta}\left(1-\frac{\delta^{2}}{5 \cdot 2 !}+\frac{\delta^{4}}{9 \cdot 4 !}-\frac{\delta^{6}}{13 \cdot 6 !}+\ldots\right)=l_{\delta}-\frac{l_{\delta} \delta^{2}}{10} \\
& T_{y}=A \sqrt{2} \bar{\delta}\left(\frac{\delta}{3}-\frac{\delta^{3}}{7 \cdot 3 !}+\frac{\delta^{5}}{11 \cdot 5 !}-\ldots\right)=\frac{l_{\delta} \delta}{3}
\end{aligned}
$$

Defining $\left(x_{4}, y_{4}\right)$ as the coordinates of the tangent-spiral (TS) point on the centertine of the highway, then 


$$
\begin{aligned}
& x_{5}=x_{4}+T_{x} \sin \Phi_{0}+T_{v} \cos \Phi_{01} \\
& y_{5}=y_{4}+T_{x} \cos \Phi_{0}-T_{y} \sin \Phi_{0}
\end{aligned}
$$

Defining $w$ as the lane width, the line from $\left(x_{5}, y_{5}\right)$ to $\left(x_{3}, y_{3}\right)$ will have an azimuth of $\Phi_{1}+90^{\circ}$ and a length of $m+w / 2$. Therefore, $\left(x_{3}, y_{3}\right)$ can be calculated as follows:

$$
\begin{aligned}
& x_{3}=x_{5}+(m+w / 2) \sin \left(\Phi_{1}+90^{\circ}\right) \\
& y_{3}=y_{5}+(m+w / 2) \cos \left(\Phi_{1}+90^{\circ}\right)
\end{aligned}
$$

5. Calculate the azimuth of the line between points 1 and $3, \Phi_{2}$.

6. For right turn curves, If $\Phi_{1}>\Phi_{2}$ (if $\Phi_{1}<\Phi_{2}$, for left turn curves), $S$ is greater than the actual sight distance (see Figure 4.3 for illustration). Decrease $S$ and repeat steps 2-5.

7. For right turn curves, If $\Phi_{1}<\Phi_{2}$ (if $\Phi_{1}>\Phi_{2}$, for left turn curves), $S$ is less than the actual sight distance (see Figure 4.3 for illustration). Increase $S$ and repeat steps 2-5.

8. If $\Phi_{1}=\Phi_{2}, S$ is equal to the actual sight distance (see Figure 4.3 for illustration). End of iterations.

Since, the azimuth is always less than $360^{\circ}$, some caution is required in the last check if, for right turn curves, $\Phi_{1}$ is slightly greater than zero and $\Phi_{2}$ is slightly less than $360^{\circ}$ (or if $\Phi_{2}$ is slightly greater than zero and $\Phi_{1}$ is slightly less than $360^{\circ}$, for left turn curves). For example, for right turn curves, if $\Phi_{2}$ is slightly less than $360^{\circ}$ and $S$ is greater than the available sight distance, $\Phi_{1}$ may be slightly greater than zer. In this case. $S$ should be decreased and another iteration is required. 


\subsubsection{General Procedure: Sight Line Passing Through Point of Intersection}

In this case. only two long straight segments are intersecting at a small deflection angle. as shown in Figure 4.4. Although no circular or spiral curves are involved in this case, it represents a possible horizontal alignment and. therefore. it is considered in this research. In general, the beginning and the end of the sight line may be lociated on any horizontal highway segment. However, the straight segments in this case are usually long enough for the beginning and the end of the sight line to be located on the two intersecting straight segments. Though, for the comprehensiveness of the research. a general iterative procedure is presented here to determine the available sight distance regardless of the beginning and end of the sight line.

As shown in Figure 4.4, the sight line will pass through the point of intersection of the two straight segments, PI. The general iterative procedure involves the following steps:

1. Determine the coordinates of the beginning of the sight line, $\left(x_{1}, y_{1}\right)$.

2. Determine the coordinates of the point of intersection (PI) at the lane centerline and at the obstruction, $\left(x_{3}, y_{3}\right)$ and $\left(x_{4}, y_{4}\right)$, respectively.

3. Determine the coordinates of the end of the sight line, $\left(x_{2}, y_{2}\right)$.

4. Calculate the area of the triangle 123 and the traverse 1324, AREAI and AREA2, respectively

5. If $A R E A I>A R E A 2, S$ is greater than the actual sight distance. Decrease $S$ and repeat steps 3 and 4. 


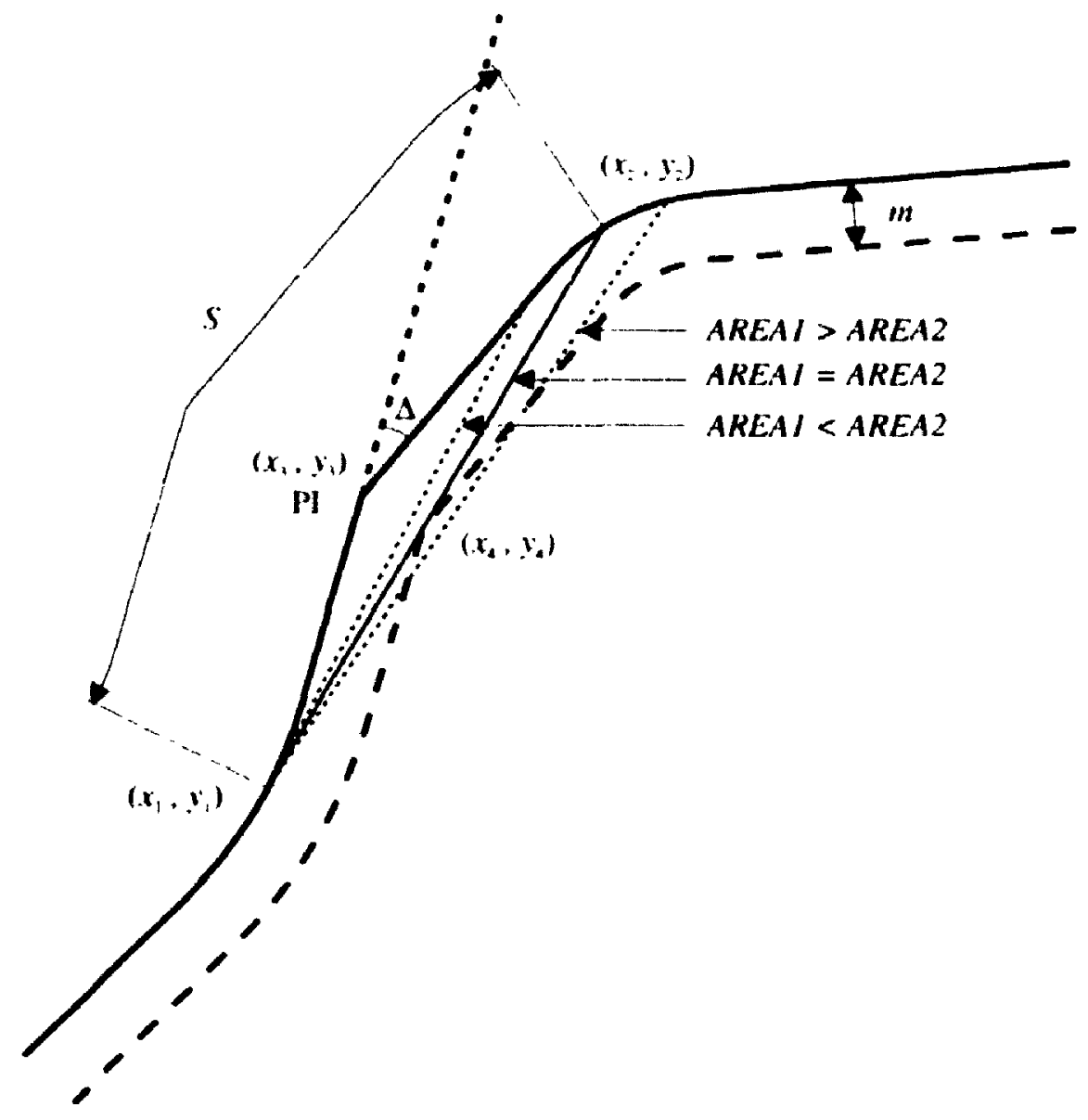

FIGURE 4.4: General Procedure: Intersecting Straight Segments Without Curves. (Continuous Obstruction)

6. If $A R E A 1<A R E A 2, S$ is less than the actual sight distance. Increase $S$ and repeat steps 3 and 4 .

7. If $A R E A I=A R E A 2, S$ is equal to the actual sight distance. End of iterations.

\section{1.4 Special Case: Simple Circular Curve}

In this case, the horizontal curve consists of a simple circular curve, having a radius $\boldsymbol{R}$, with two straight segments (tangents) at the two ends. Obviously, the sight line can only 
presented previously can be applied. However, other closed form relationships have heen developed for this special case to determine the available sight distance more casily and accurately. These formulas can be considered extensions to the work of Fasa (I9) la) which considered only a single (or multiple) lateral obstruction.

For the case of a simple curve, there are four possibilities regarding the beginning and the end of the sight line touching the obstruction:

(i) Sight line begins on first tangent and ends on curve.

(ii) Sight line begins on first tangent and ends on second tangent.

(iii) Sight line begins and ends on curve.

(iv) Sight line begins on curve and ends on second tangent.

Case (i): Sight line begins on first tangent and ends on curve. As shown in lïigure 4.5a,

$$
l_{2}=\left(R^{2}+l_{1}^{2}\right)^{1 / 2}
$$

where $l_{1}$ is the distance between the driver and the point of curve (PC). 


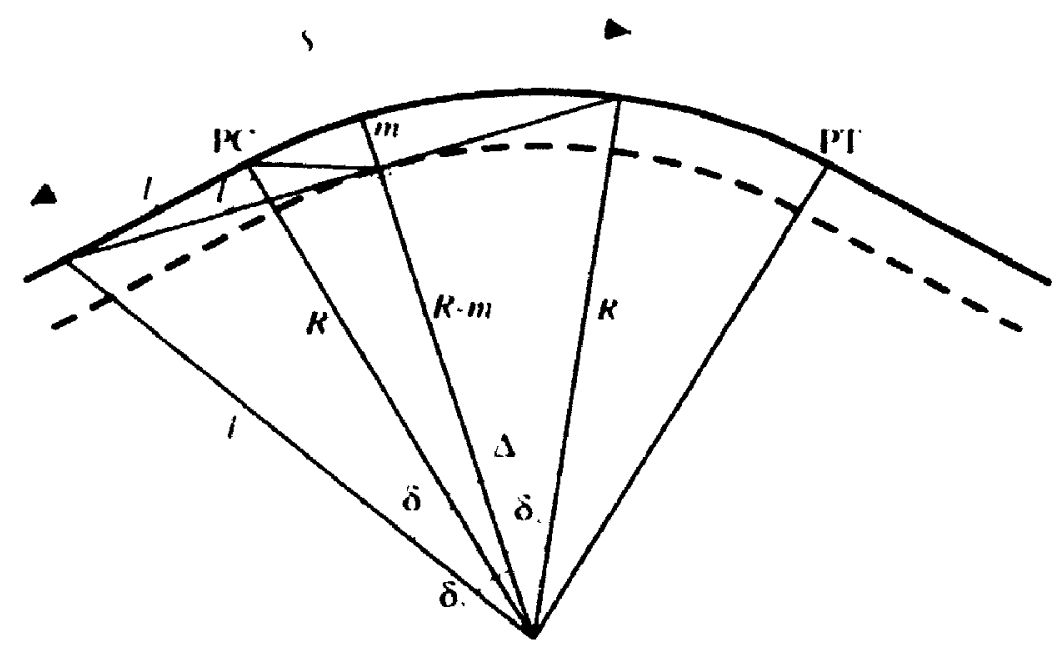

(a) Sight Line Begins on Tangent and Ends on Curve.

s

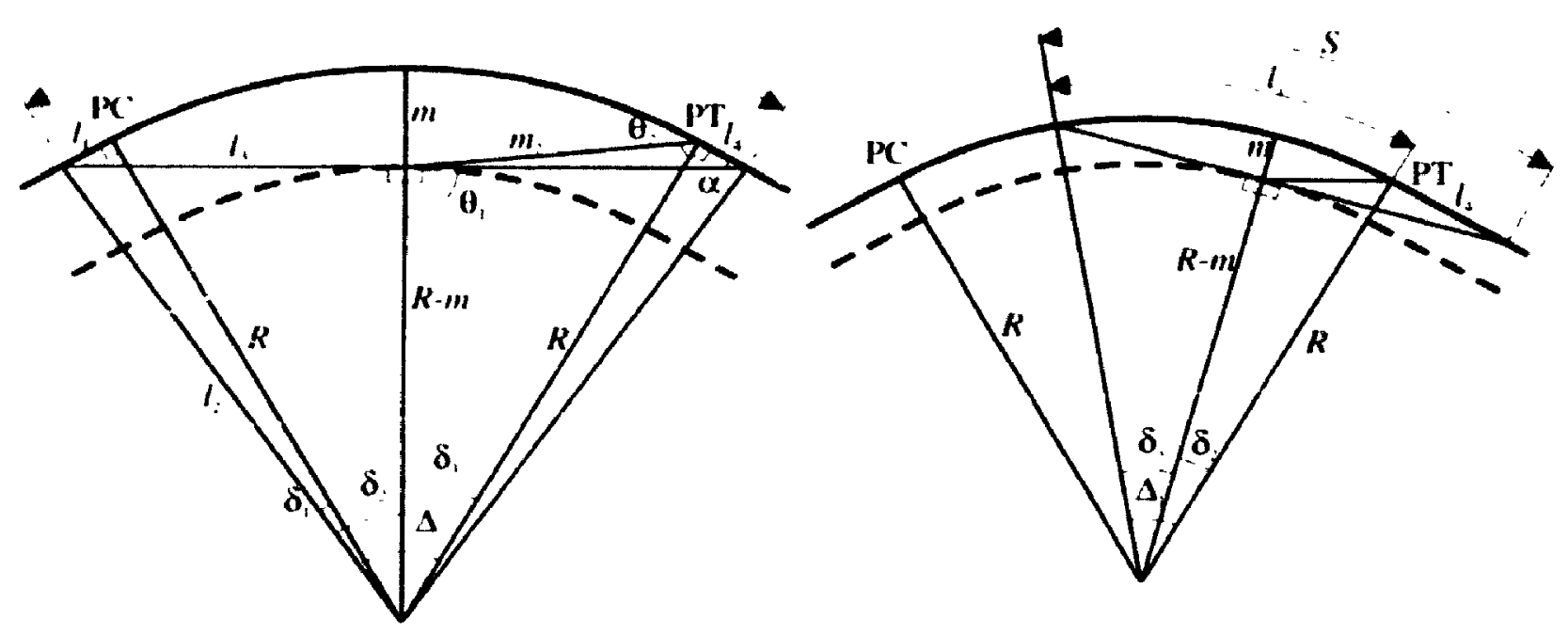

(b) Sight Line Begins and Ends on Tangents. (c) Sight Line Begins on Curve and Ends on Tangent.

FIC URE 4.5: Special Case: Simple Horizontal Curve. (Continuous Obstruction) 


$$
\begin{aligned}
& \delta_{1}=\sin ^{-1}\left(l_{1} / R\right) \\
& \delta_{2}=\cos ^{-1}\left(\frac{R-m}{l_{2}}\right) \\
& \delta_{3}=\cos ^{-1}\left(1-\frac{m}{R}\right) \\
& \Delta_{1}=\delta_{2}-\delta_{1}+\delta_{3}
\end{aligned}
$$

Then, the available sight distance is:

$$
S=l_{1}+R \Delta_{1} \times \frac{\pi}{180^{\circ}}
$$

Case (ii): Sight line begins on first tangent and ends on second tangent. $\Lambda$ s shown in Figure 4.5b, $\delta_{1}$ and $\delta_{2}$ can be determined as in case (i). Defining $\Delta$ as the total deflection angle of the curve, then:

$$
\begin{aligned}
& \delta_{3}=\Delta+\delta_{1}-\delta_{2} \\
& m_{2}=\left(R^{2}+(R-m)^{2}-2 R(R-m) \cos \delta_{3}\right)^{1 / 2} \\
& \theta_{1}=\sin ^{-1}\left(R \sin \delta_{3} / m_{2}\right) \\
& \theta_{2}=\sin ^{-1}\left[(R-m) \sin \delta_{3} / m_{2}\right] \\
& \alpha=180-\theta_{1}-\theta_{2}
\end{aligned}
$$




$$
l_{4}-m_{2} \sin \left(\theta_{1}-90^{\circ}\right) / \sin \alpha
$$

Then, the available sight distance is:

$$
S-l_{1}+R \Delta \times \frac{\pi}{180^{\circ}}+l_{4}
$$

Case (iii): Sight line begins and ends on curve. In this case, the formula presented by AASHTO can le applied as follows:

$$
S-2 R \times \cos ^{-1}(1-m / R) \times \frac{\pi}{180^{\circ}}
$$

Case (iv): Sight line begins on curve and ends on second tangent. The relationships involved in this case are backward derivations for case (i). As shown in Figure 4.5c, the angle $\delta_{3}$ can be calculated as in case (i). Knowing the distance $l_{1}$ and the radius $R$, the angle $\Delta_{1}$ and subsequently $\delta_{2}$ can be calculated. Then, $l_{4}$ can be calculated as in case (ii). Finally, $S$ equals the sum of $l_{1}$ and $l_{4}$.

\subsubsection{Special Case: Intersecting Long Straight Segments}

This case, as shown in Figure 4.6, is a special case of sight line passing through point of intersection where two straight segments are intersecting at a deflection angle $\Delta$. The straight segments are long enough for the beginning and end of the sight line to be located at the first and second segment. respectively. The driver is at a distance $l_{\mathrm{t}}$ from 
PI. As shown. the sight line will touch the continuous obstruction at its PI. lirom Figure 4.6.

$$
\begin{aligned}
& l_{3}-m / \tan \frac{180^{\circ}-1}{2} \\
& \alpha=\tan ^{-1}\left[m /\left(l_{1}-l_{2}\right)\right] \\
& \beta=\Delta-\alpha \\
& l_{2}=l_{1} \sin \alpha / \sin \beta
\end{aligned}
$$

Then, the available sight distance is:

$$
S=l_{1}+l_{2}
$$

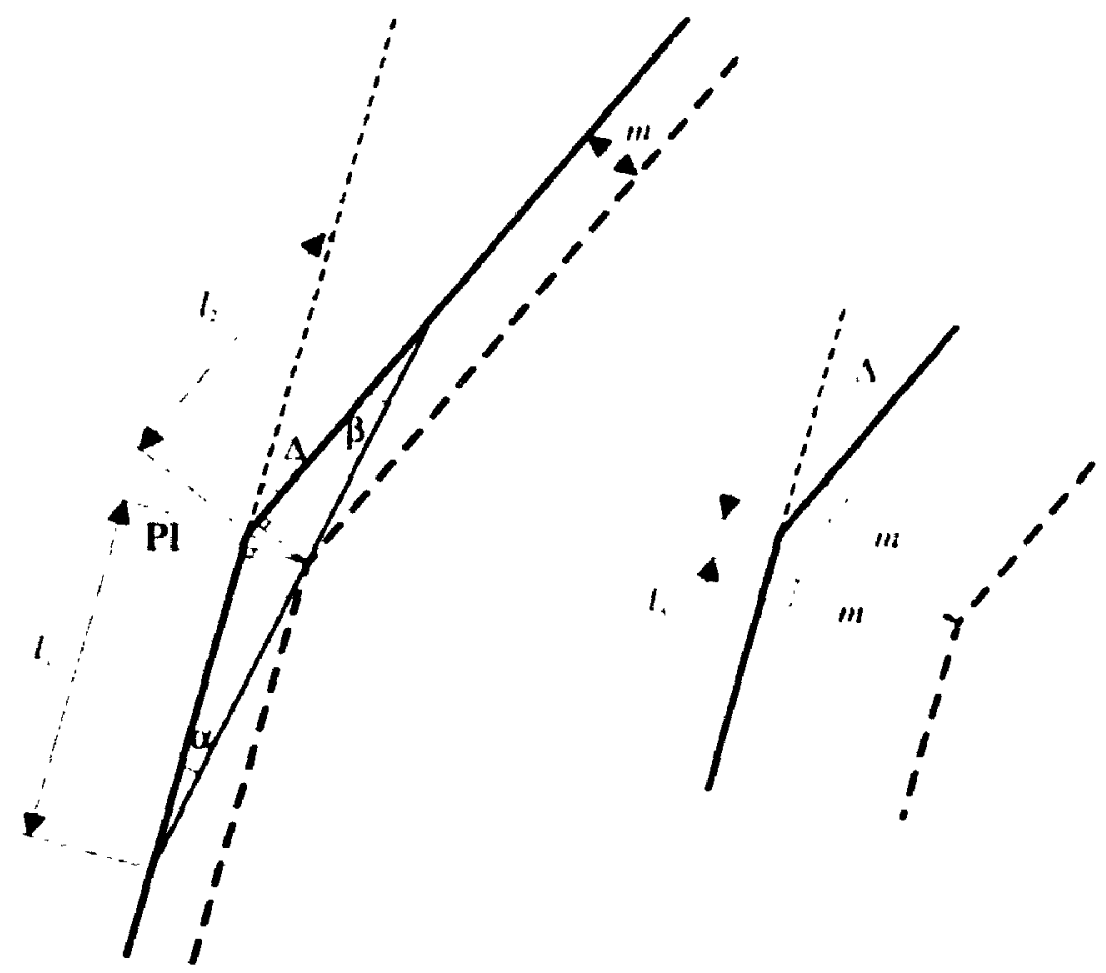

FIGURE 4.6: Special Case: Intersecting Straight Segments Without Curves. (Continuous Obstruction) 


\subsection{Horizontal Alignment with Single Obstructions}

As mentioned previously, the case of a single obstruction has been extensively studied hy many researchers. Formulas relating the available sight distance to the lateral clearance on simple horizontal curves already exist. In this research. two general itcrative procedures have heen developed to check the available sight distance regardless of the components of the horizontal curve and the positions of the beginning and end of the sight line.

The first procedure uses the areas as explained in the general procedure of sight line passing through point of intersection while the second procedure uses the azimuths of the lines in a way similar to the general procedure of the continuous obstruction with the sight line tangent to a spiral curve. In the first procedure, the coordinates of an intermediate point on the highway between points 1 and 2 can replace the coordinates $\left(x_{3}\right.$, $\left.y_{1}\right)$ and the coordinates of the obstruction are used instead of $\left(x_{4}, y_{4}\right)$. In the second procedure, the coordinates of the obstruction are directly used instead of the point of tangency $\left(x_{3}, y_{3}\right)$.

\subsection{Vertical Alignment}

Generally. vertical alignment may obstruct the sight line in three possible ways. First, if the sight line from the driver eye to the object should pass over a crest curve, it may intersect with the road itself. The points of intersection may be on a tangent segment, a crest curve, or a sag curve. Subsequently, the sight distance is limited by having the sight line tangent to the crest curve. Second. although sag curves do not represent a 
sight obstruction at daytime, the availahle SSD at nighttime is limited to the farthest puint covered by the vehicle headlights. However, this restriction is nut applied for the available PSD since the driver can always detect the opposing vehicles by their uwn headlights. Therefore, the case of headlight sight distance is heyond the sione of this research. Third, overpasses existing on sag curves may obstruct the sight limk which must be limited by passing under these overpasses.

In considering the separate vertical alignment, as in the general practice it highway vertical alignment design. the protile of the highway is drawn using a system of axes $X-Z$ with the first axis going through the highway centerline. Therefiore. the coordinates of any point $(x, z)$ represent the point station and elevation, respectively. It should be noted also that, in the following sections. the grade of a tangent wegnkent or the instantaneous grade at a point on a curve is used as a percentage and referred 10 as $g$. The sign of $g$ is positive if the grade is upward and negative if it is downward. The algebraic difference in grades of vertical curves, which is always a prsilive value, is used as a percentage and referred to as $A$. Finally. $h_{1}$ and $h_{2}$ are referring w the heights ot the driver's eye and the object, respectively.

\subsubsection{Geometric Characteristics of Vertical Alignment Elements}

Elements' Equations. Three main elements are encountered when studying sight distance on vertical alignments at daytime. These elements are the sight line, tangent segments, and sag and crest vertical curves. 
The sight line is a straight line whose equation is:

$$
\frac{z-z_{1}}{x-x_{1}}-\frac{z_{z}-z_{1}}{x_{2}-x_{1}}
$$

where $\left(x_{1}, z_{1}\right)$ and $\left(x_{2}, z_{2}\right)$ are the coordinates of any two points on the sight line and will he taken in this research as its heginning and end. respectively.

The previcus equation can he simplified as:

$$
=-c_{1} x+c
$$

where

$$
\begin{aligned}
& c_{1}-\frac{z-z_{1}}{1 z-x_{1}} \\
& c_{2}-z_{1} x_{1}
\end{aligned}
$$

Tangent segments are also straight lines. and can be represented by Equation 4.33 or 4.34 hut with $\left(x_{1}, z_{1}\right)$ and $\left(x_{2}, z_{2}\right)$ representing the coordinates of any two points on the segment. However, to distinguish hetween the equations of the sight line and that of a tangent segment. the lia. it will be written as:

$$
z-c_{1} x+c_{4}
$$

It can be noted that since $c_{3}$ in Equation 4.36 represents the slope of the line, it can be taken directly as the decimal grade of the segment. $g / 100$.

Highway vertical curves are always taken as second degree parabolas. If the coordinate origin is taken at the point of beginning of vertical curve (BVC). the curve equation can be written as: 
for a crest cume: $=-\frac{g_{1}}{1(x)},-\frac{t}{2(x) I},:$
for a sag cune: $=-\frac{g_{1}}{1(x)},+\frac{A}{2(x) I},:$

where

$$
\begin{aligned}
& g_{1}=\text { grade of the first tangent. } \\
& A=\text { algebraic difference in grades of the curve. and } \\
& L \quad=\text { curve length. }
\end{aligned}
$$

Using an arbitrary origin of the coordinate system so that the corrdinates of the BVC are $\left(x_{1},-2\right)$. Equation 4.37 can be written as:

$$
z=c_{5} x^{2}+c_{n} x+c
$$

where

$$
\begin{aligned}
& c_{a}=-\frac{A}{200 L} \quad \text { (crest cune) } \\
& c_{4}=+\frac{A}{200 L} \quad \text { (sag cune) } \\
& c_{6}=\frac{g_{1}}{100}-2 c_{4} x_{1} \\
& c_{7}=z_{1}-\frac{g_{1}}{100} x_{1}+c_{5} x_{1}^{2}
\end{aligned}
$$

Intersection with Sight Line. As will be shown later. determuning lixe punt(b) ot. intersection between the sight line and the vertical alignment elements is a key step in the procedure developed for determining the available sight distance. If the clemint 
considere' is a tangent segment, it can have only one point of intersection with the sight line. Solving Equations 4.34 and 4.36, the $X$-coordinate of the point of intei section , $x_{i}$ can be written as:

$$
x_{1}=\frac{c_{4}-c_{2}}{c_{1}-c_{3}} \quad\left(c_{1} \neq c_{3}\right)
$$

It should $b_{1}$ - noted that if $c_{1}=c_{3}$, the two lines are either parallel or coinciding. In either case, this specific segment does not obstruct the sight line.

If the element is a curve, it can nave two points of intersection with the sight line. Solving Equations 4.34 and 4.38 , these points can be determined as:

$$
x_{1}=\frac{\left(c_{1}-c_{6}\right) \pm \sqrt{M}}{2 c_{5}} \quad(M \geq 0)
$$

where

$$
M=\left(c_{n}-c_{1}\right)^{2}-4 c_{5}\left(c_{7}-c_{7}\right)
$$

However, the sign of $M$ should be checked first before applying Equation 4.43. If $M<0$, there are two imaginary points of intersection; i.e., the curve does not intersect with the sight line. If $M>0$, there are two real points of intersection whose $X$-coordinates can be determined using Equation 4.43. Finally, if $M=0$, the two points of intersection will coincide and represent a single point of tangency. This last case is the limiting condition for the sight distance and occurs on crest vertical curves only. 


\subsubsection{Road as Sight Obstruction}

As stated previously, the road itself may obstruct the sight line if the latter should pass over a crest curve. As shown in Figure 4.7, for such obstruction to happen, there must be at least two points of intersection between the sight line and the highway segments within the limits of the sight line. Although the road cannot obstruct the sight line unless there is a crest curve, the points of intersection may be on the crest curve, a sag curve, or a tangent segment. The procedure developed in this research to deteimine the available sight distance is carried out assuming an initial value for the sight distance, $S$. Then, any existing intersection between the highway segments and the sight line is checked, and $S$ is increased or decreased until the sight line becomes a tangent to a crest curve. The following steps explain how the procedure can be carried out.

1. Determine the coordinates of the beginning of the sight line, $\left(x_{1}, z_{1}\right)$.

2. Determine the coordinates of the end of the sight line, $\left(x_{2}, z_{2}\right)$.

3. Establish the equation of the sight line as in Equation 4.34.

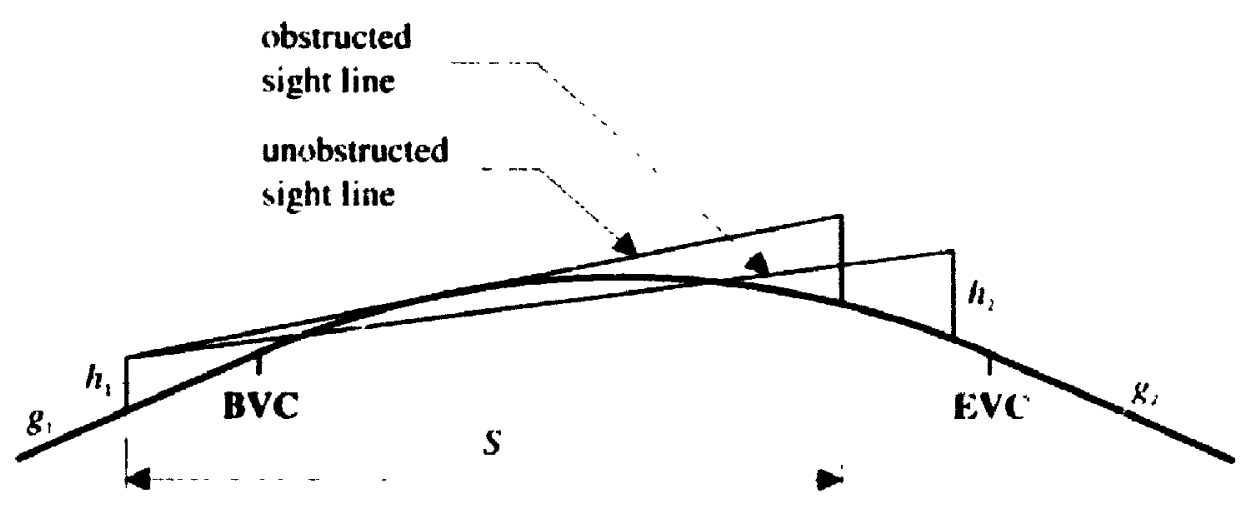

FIGURE 4.7: Sight Distance Limited by Crest Vertical Curve. 
4. Check the existence of any point of intersection between the sight line and all the highway segments between the beginning and end of the sight line.

5. If ANY segment intersects with the sight line within the segment limits, $S$ is greater than the actual sight distance. Decrease $S$ and repeat steps 2-4.

6. If NO segment intersects with the sight line within the segment limits, $S$ is less than the actual sight distance. Increase $S$ and repeat steps $2-4$.

7. If the sight line is tangent to a crest curve, $S$ is equal to the actual sight distance. End of iterations.

\subsubsection{Sight-Hidden Dips}

As shown in Figure 4.8, sight-hidden dips may exist if a crest curve is followed by a sag curve (with or without an intermediate tangent segment). Using the model developed by Easa (1994b), the existence of the sight-hidden dips can be checked and its length can be determined. However, a simple iterative procedure, easily programmable, is presented here to check the existence of a sight-hidden dip and to determine the available sight distance 1 such cases. This procedure should follow the determination of the

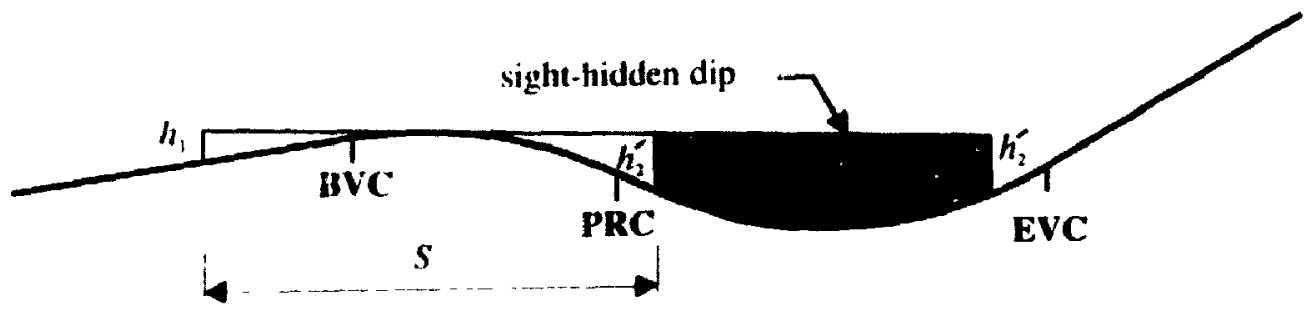

FIGURE 4.8: Development of Sight-Hidden Dips. 
farthest point seen by the driver as explained in the previous section. The procedure involves the following steps:

1. Determine the unobstructed sight distance, $S$, as explained previously.

2. Establish the equation of the sight line as in Equation 4.34 .

3. For a number of points between the two limits of the sight line and taken at a fine step, determine the vertical clearance between the sight line and the highway surface. The clearance at a certain point can be obtained as the elevation ohtained from Equation 4.34 minus that obtained from Equation 4.36 or 4.38 , depending on the segment type.

4. Determine the maximum clearance, $z_{h}$, and the station at this point, $x_{h}$.

5. If $z_{h} \leq h_{2}$, NO sight-hidden dip exists. End of procedure.

6. If $z_{h}>h_{2}$, check the existence of any point of intersection between a sight line from the driver eye to an onject at $x_{h}$ and the different highway segments.

7. If there is NO point of intersection, no sight-hidden dip exists. End of procedure.

8. If there is ANY point of intersection, a sight-hidden dip exists. Take $S=x_{h}-x_{1}$ and repeat the procedure in Section 4.3.2.

It should be noted that if a sight hidden dip is detected, the height of the object may be reduced by a factor called the visibility factor, $f_{v}$. The use of this factor was recommended by Easa $(1994 b)$. The object height used in this case will he, $h_{2}^{\prime}=(1$ $\left.f_{v}\right) h_{2}$, where $f_{v}=0.1$ to 0.3 . 


\subsubsection{Overpasses as Sight Obstructions}

As shown in Figure 4.9, the existence of overpasses on sag curves may obstruct the sight line. To check the existence of such an obstruction, the elevation of the sight line at the station of the overpass is compared to the elevation of the lowest point in the overpass. If the elevation of the sight line is higher than that of the overpass, an obstruction exists. $S$ should be reduced and another iteration is performed until the elevation of the sight line is lower than that of the overpass. However, it should be noted that the sight line in this case should be taken as the line connecting the driver's eye to the minimum height required for the object to be detected, $h_{\operatorname{man}}$. Although this height may be theoretically set as zero, it is preferred to be of a certain minimum height. In determining the available PSD, and because drivers use the headlight of the opposing vehicles to detect them at night, it is recommended here to take this minimum height as the height of the headlight. Finally, if the overpass is relatively wide, the sight line may be obstructed by the two bottom edges of the overpass. In this case, the obstruction should be modelled by two points over the highway profile.

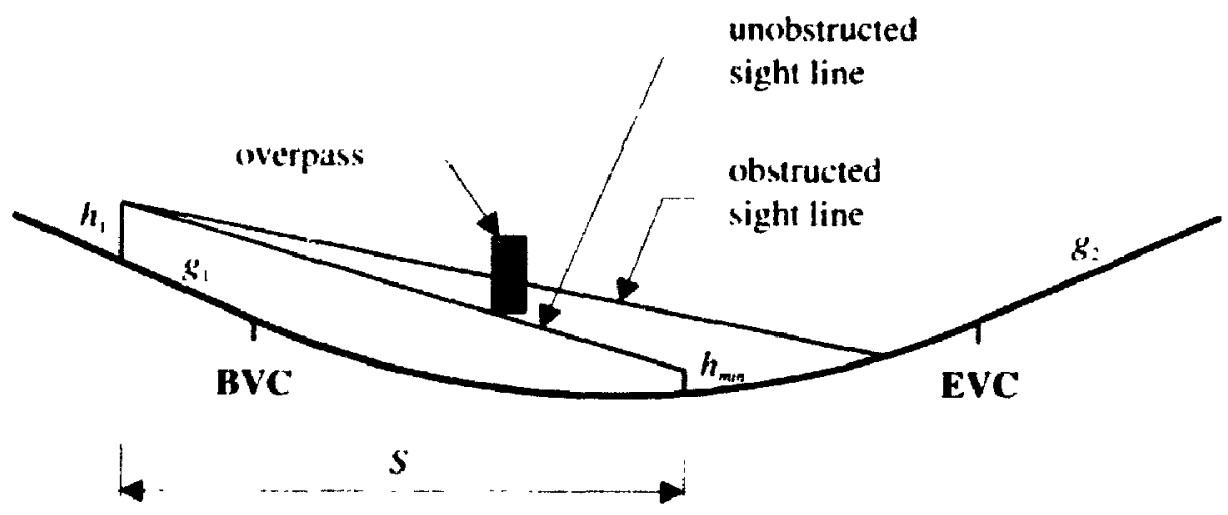

FIGURE 4.9: Sight Distance Limited by Overpass on Sag Vertical Curve. 


\subsection{Unified Methodology for Separate Horizontal and Vertical Alignments}

In the models presented above. each type of sight obstructions is dealt with in a unique way, and thus, computer programming is not an easy task. Also, in dealing with continuous obstructions on horizontal alignments, the lane width has to be constant. As a result, lane widening could not be considered. In addition. spline grades in vertical alignments, where the alignment is given as stations and elevations, do not have an explicit mathematical equation and cannot he modelled. More importantly, it is extremely difficult to extend these models to 3-D combined alignments. In this section an analytical model that can deal with separate 2-D alignments and can overcume these disadvantages is presented. The model is an application of the linite element method where sight obstructions are modelled using finite elements, and the intersection between the sight line and these elements is checked to determine the available sight distance.

In the following sections. a background for the parametric elements in 2-1) alignments, upon which the model is based, will be presented. Then, the model itself will be explained. It should be noted that the coordinates of any point will be referred to as $(x, y)$ which represent the east and north coordinates of the point (in horizontal alignments) or the station and elevation of the point (in vertical alignments). The reference point can be anywhere because the sight distance is governed by the relative, not the absolute, positioning of the points. 


\subsection{Background to Parametric IElements}

The model developed here depends on the characteristics of the parametric elements which have been used in the structural analysis using the finite element method since the 1960's ( Cook et al, 1989; Zienkiewicz and Taylor, 1991). The basic idea in using the parametric elements is to transform distorted straight or curved lines (in a global coordinate system, $X-Y$ ) to regular one-dimensional straight lines (in a local coordinate system, $\eta$ ) using a number of points with known coordinates (nodes). The coordinates of "ny point within the element can be interpolated among the nodes using interpolation functions. These functions along with a specific number of nodes can define a unique element geometry (shape), and therefore they are referred to as shape functions. The interpolation in 2-D can be expressed as:

$$
\begin{aligned}
& x=\sum_{i=1}^{n} N_{i} x_{1} \\
& y=\sum_{i=1}^{n} N_{1} y_{1}
\end{aligned}
$$

where

$$
\begin{aligned}
& n=\text { total number of element nodes. } \\
& x_{1} y=\text { global cartesian coordinates of any point, } \\
& x_{1}, y_{1}=\text { global cartesian coordinates of node } i, \text { and } \\
& N_{1}=\text { shape function of node } i .
\end{aligned}
$$

The shape functions, $N_{1}$, for an element with a specific number of nodes can be obtained by Gauss interpolation. For example, referring to Figure 4.10, for a 2-node 
element with its nodes at the local coordinates $\eta$ of -1 and +1 . respectively. the shape functions will be:

$$
\begin{aligned}
& N_{1}=\frac{1}{2}(-\eta+1) \\
& N_{2}=\frac{1}{2}(\eta+1)
\end{aligned}
$$

Similarly, the 3-node element shown in Figure 4.10 (with its modes at the lix:al coordinates $\eta$ of $-1,0$, and +1 , respectively) will have shape functions ats follows:

$$
\begin{aligned}
& N_{1}=\frac{1}{2}\left(\eta^{2}-\eta\right) \\
& N_{2}=-\left(\eta^{2}-1\right) \\
& N_{1}=\frac{1}{2}\left(\eta^{2}+\eta\right)
\end{aligned}
$$

As shown in Equation 4.46, the shape functions of the 2-node element are lincar. As a result. applying Equation 4.45 to calculate the coordinates of any point in the element will represent a linear interpolation between the two nodes. This elenkent can exactly model straight lines only, and is referred to here as the linear cle'me'nt. On th: other hand, the shape functions of the 3-node element are quadratic functions, and therefore such an element can model exactly second-degree parabolic curves. This element is referred to as the quadratic element. Also, higher order elements wilh higher number of nodes can be used to model higher degree curves. 


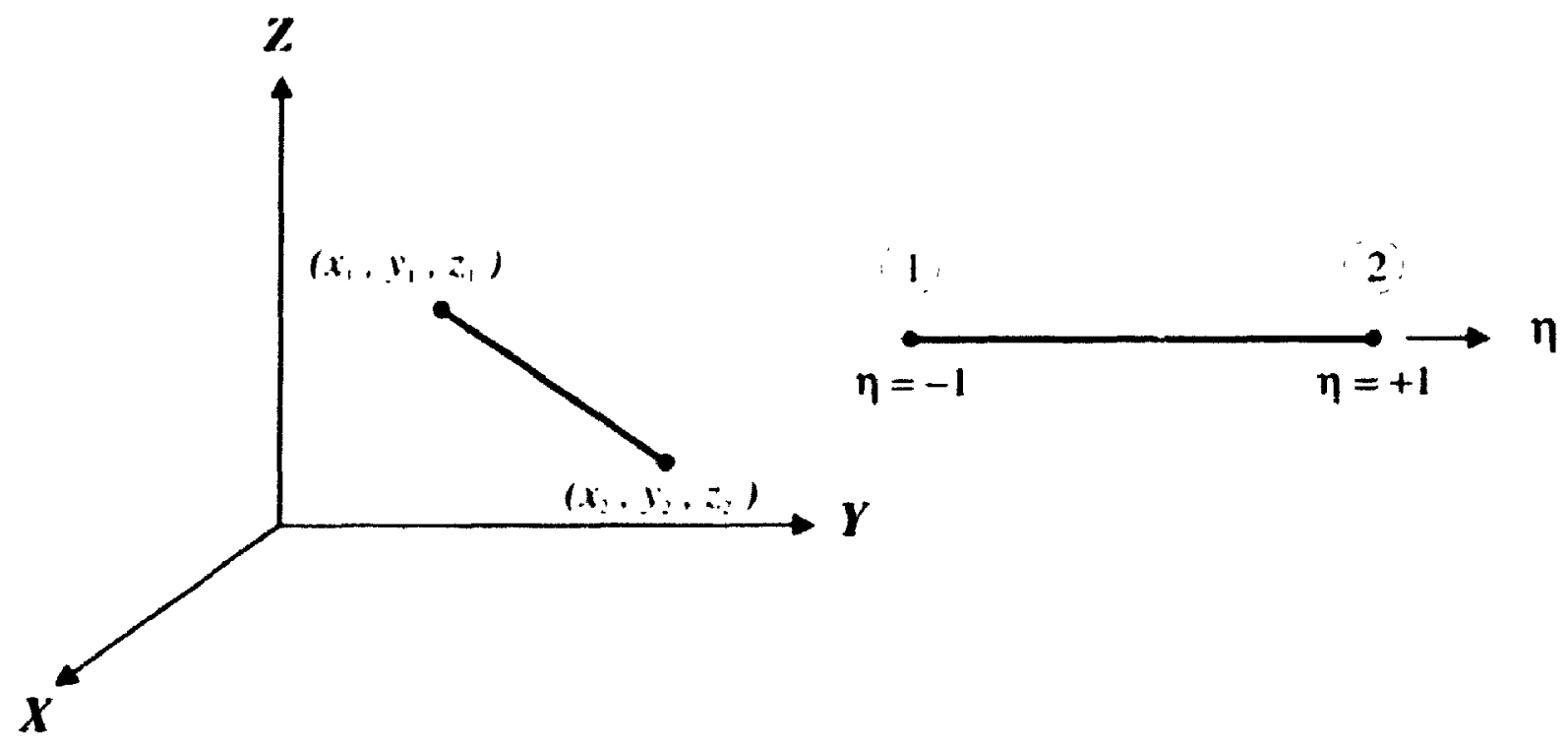

(a) 2-Node Lincar Element.

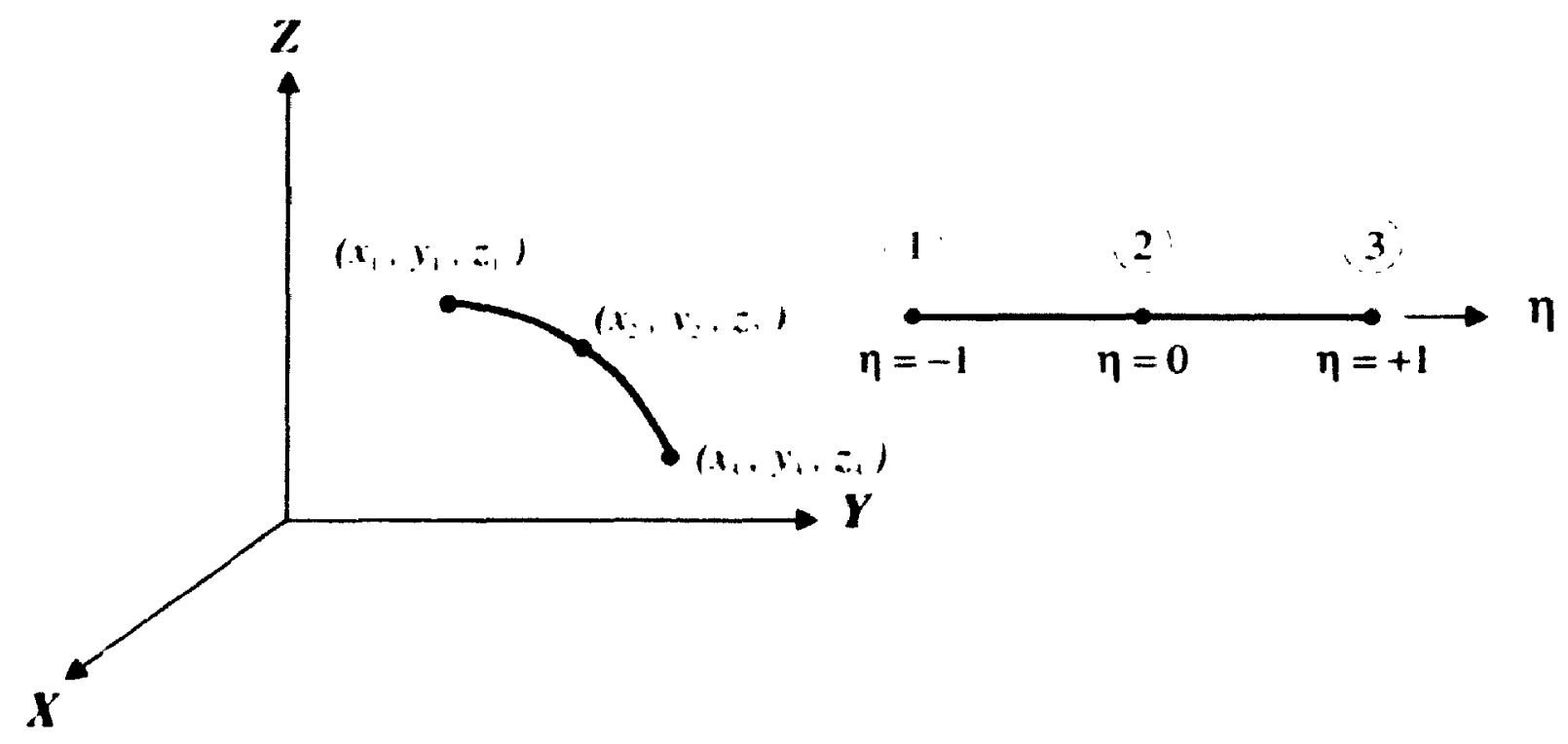

(b) 3-Node Quadratic Element.

FIC (IRE 4.10: Parametric Elements for 2-D Horizontal and Vertical Alignments. 


\subsubsection{Alignment Idealization}

According to this model. sight obstnctions in horizontal and vertical alignments are idealized into a series of parametric elements. The elements which can the used for each sight obstruction are as follows:

Horizontal Nlignment. As shown in Figure 4.11. the different types of sight obstructions modelled in horizontal alignments are:

1. Continuous obstructions on straight segments (tangents) are straight lines, and therefore they are exactly modelled using linear elements.

2. Although the equation of horizontal spiral curves is an infinite series, it is usually approximated to a second degree equation only. Therefore, continuous obstructions parallel to spiral curves are modelled using quadratic elements. Ilowever, the smaller the element size, the higher the modelling accuracy. Therefore, the elements used to model continuous obstructions on spiral curves will be referred 10 as short quadratic elements.

3. Circular curves are not parabolic, and therefore cannot be exactly modelled using the quadratic element. However, since they are second degree curves, they call be reasonably modelled using short quadratic elements. Generally, the smaller the element size the higher the accuracy and the more the time required to determine the available sight distance. Although more accurate modelling can be achieved by increasing the number of nodes in the element, such elements are not used here because of the difficulty expected in using such elements in 3-D analysis. As a 
2-node element modelling

ond-pount ungle obstructuon

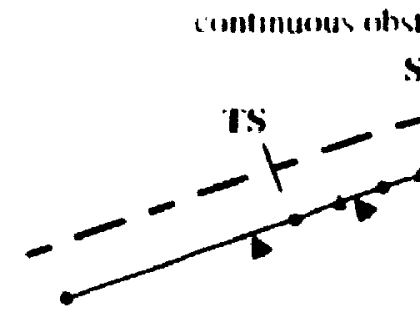

- An cond mixde.

- An internectadte node.

(a) Continuous and One-Point Single Obstructions.
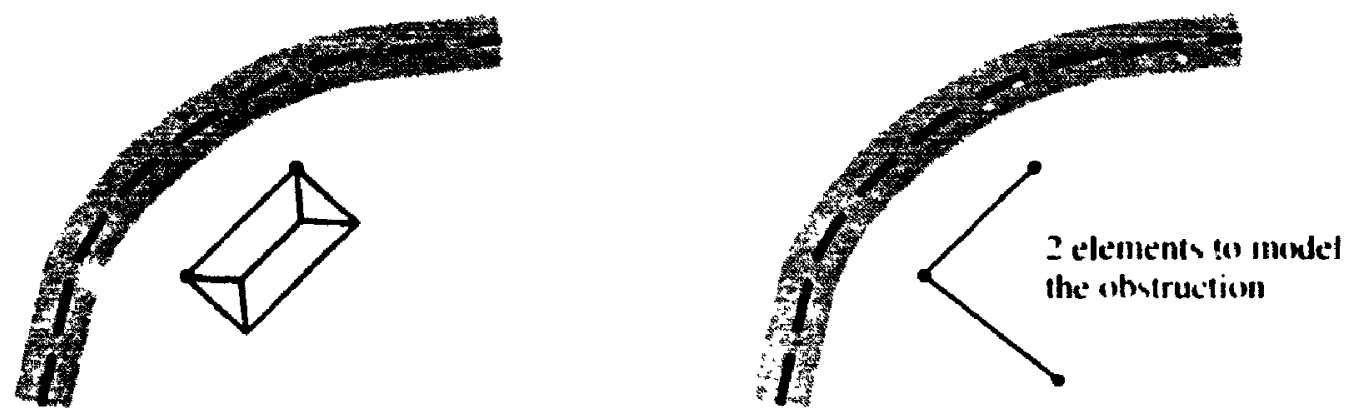

(b) Two-Point Obstruction
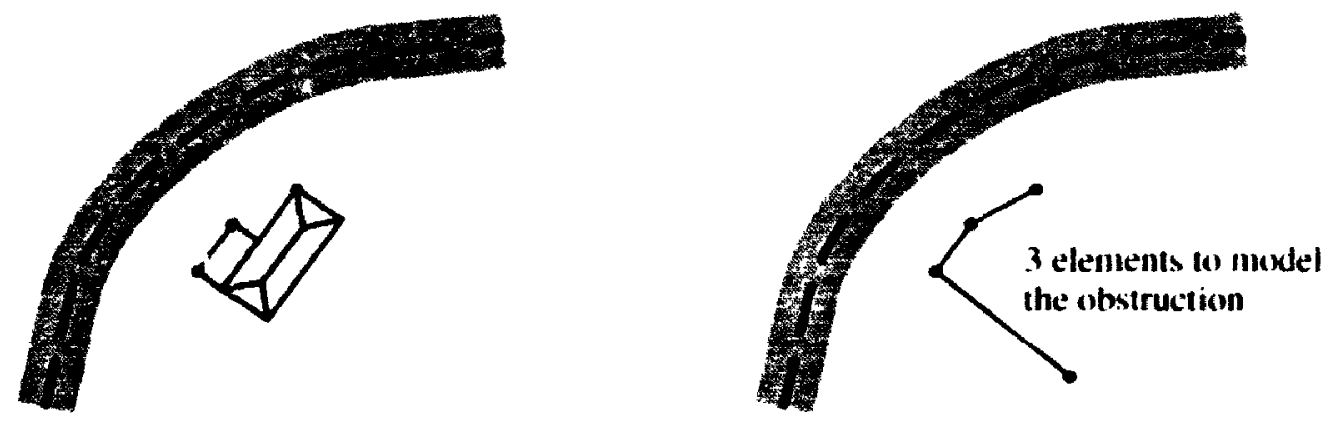

(c) Three-Point Obstruction

FIGURE 4.1 1: Idealization of Horizontal Alignment. 
result, continuous obstructions parallel to circular curves are modelled using a number of short quadratic elements.

4. Single obstructions. although defined by a sing!e moint, are represented by a limear element. The first node of the element is the poimt defining the obstruction while the element extends away from the highway. Sometimes, the ohstruction has considerably large dimensions and cannot be modelled properly as a single point but rather with two or three points. In this case, a number of elements should he used as shown in Figure $4.11 b$ and $c$.

Vertical Alignment. As shown in Figure 4.12. the different types of sight obstructions modelled in vertical alignments are:

1. Straight segments (slopes) are straight line, and therefore they are exactly modelled using linear elements.

2. Vertical curves are parabolic curves, and therefore, they are exactly modelled using a quadratic element for each curve, regardless of its length. These elements will be referred to as long quadratic elements.

3. In some cases, vertical curves are not perfect parabolas but rather spline grades defined by the station and elevation of a number of points on the curve. This type of curvature does not have an explicit mathematical formula and cannot be considered in the model presented in Section 4.3. However, in using the parametric elements. spline grades can be modelled using short quar ratic elements similar to horizontal curves. 


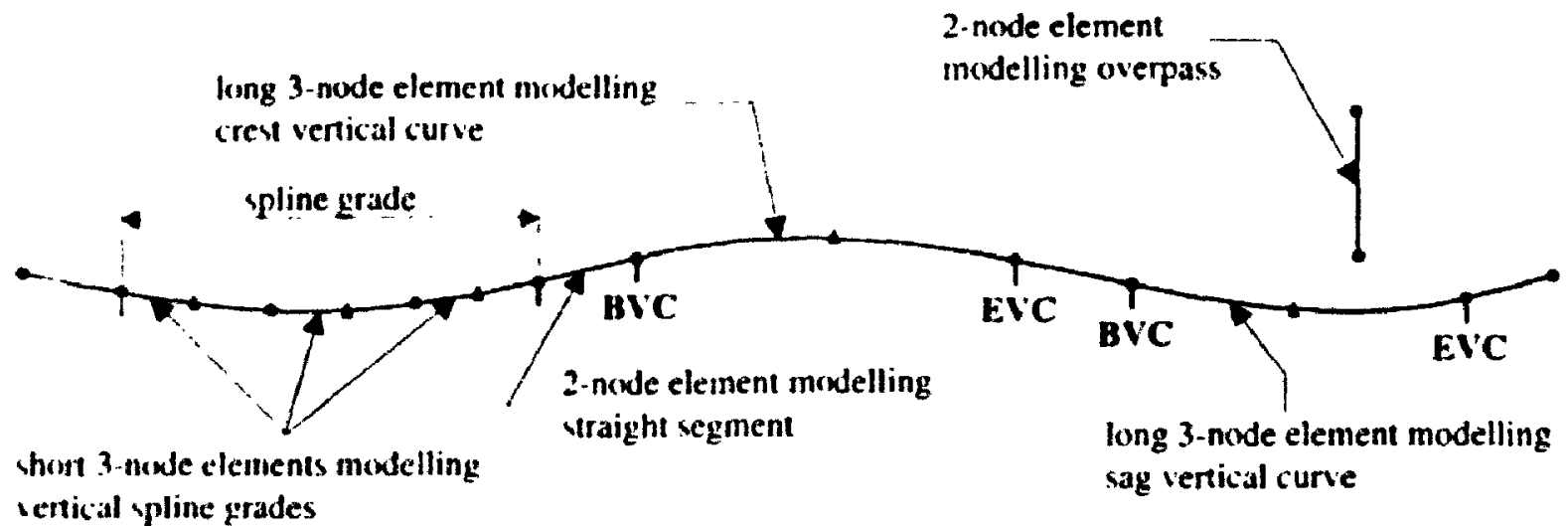

- An end ninde.

- An intermediate node.

\section{FIGURE 4.12: Idealization of Vertical Alignment.}

4. Overpasses are modelled similar to the single obstructions in horizontal alignments. This is by using a linear element with the first node at tue lowest point of the overpass, and the element extends up. As mentioned previously, if the overpass is relatively wide, two consecutive elements, at the limits of the overpass, should be used in modelling the obstruction.

\subsubsection{Intersection Berween Sight Line and Parametric Elements}

As will be explained later. the model depends mainly on the intersection between the sight line and the highway elements, mapped into linear and quadratic parametric elements. In this section. $\left(x_{31}, y_{11}\right)$ and $\left(x_{32}, y_{22}\right)$ refer to the cartesian coordinates of any two points on the sight line. Usually, these two points are taken as its beginning and end. Similarly, $\left(x_{1}, y_{i}\right)$ refer to the cartesian coordinates of node $i$. The parameter $a$, refers to 


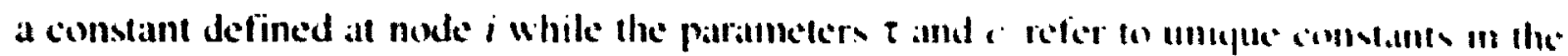
equation.

In a 2-D alignment. the equation of the yght linc is

$$
\frac{x-x_{1}}{y-y_{1}}=\frac{r_{1}-y_{1}}{y_{1}-y_{1}} \quad\left(y_{1}+y_{1}\right)
$$

or

$$
r-s_{11} r+\left(S_{11} r_{1,}-1,1=0 \quad 1 r_{0}+r_{1,1}\right.
$$

where

$$
S_{11}=\left(1_{12}-1_{1,1}\right)\left(y_{12}-x_{1 j}\right)
$$

If $y_{1}=y_{2}=y_{1}$, the equation of the sight lime hecomes:

$$
y-y=0 \quad\left(y=y_{1}=1,1\right.
$$

Since the point(s) of intersection between the sight line and the elemeant must sittsly the equations of both. then

$$
\sum N_{1} a_{1}+\tau=0
$$

where

$$
\begin{aligned}
& u_{1}=r_{1}-S_{2} v_{1} \\
& \left(v_{11} \neq v_{12}\right) \\
& u_{1}=y_{1} \\
& \left.w_{11}=v_{n}\right) \\
& \tau=-x_{11}+S_{11} y_{1} \\
& \left(v_{1} \neq v\right) \\
& t=-y \\
& \left(y_{11}=v_{1 .}\right)
\end{aligned}
$$


Equation 4.51 represents a polynomial in one variable, $\eta$. which can be obtained by substituting for the values of $N_{1}$ as shown in Equations 4.46 and 4.47. For a linear element, Equation 4.51 can be written in terms of $\eta$ as follows:

$$
c_{1} \eta+c_{2}=0
$$

where

$$
\begin{aligned}
& c_{1}=-a_{1}+a_{2} \\
& c_{2}=a_{1}+a_{2}+2 \tau
\end{aligned}
$$

The solution of Equation 4.52 may produce:

1. No solution, if the equation is trivial $\left(c_{1}=c_{2}=0\right)$. This case can happen only if the sight line is parallel to or coincident with the linear element. As a result, no sight obstruction exists.

2. One real root, $\eta=-c_{2} / c_{1}$. In this case, one point of intersection exists whose cartesian coordinates can be calculated using Equation 4.45.

If the element considered is quadratic. Equation 4.51 can be written in terms of $\eta$ as foilows:

$$
c_{1} \eta^{2}+c_{2} \eta+c_{3}=0
$$

where

$$
\begin{aligned}
& c_{1}=a_{1}-2 a_{2}+a_{1} \\
& c_{2}=-a_{1}+a_{3} \\
& c_{1}=2 a_{2}+2 \tau
\end{aligned}
$$


The solution of Equation 4.53 may produce:

1. No solution, if the equation is trivial $\left(c_{1}=c_{2}=c_{1}=0\right)$. No sight obstruction exists.

2. Two imaginary roots, if $c_{2}^{2}-4 c_{1} c_{3}<0$. This case also represents mo intersection.

3. One real root, if $c_{1}=0$ and $c_{2} \neq 0$. In this case, one point of intersection exists whose cartesian coordinates can be calculated using Equation 4.45 .

4. Two different real roots, if $c_{2}^{2}-4 c_{1} c_{1}>0$. In this case, two points of interscelion exist, and their cartesian coordinates can be calculated using Equation $\mathbf{4 . 4 5}$.

5. Two identical real roots, if $c_{2}^{2}-4 c_{1} c_{1}=0$. In this case, the two points of intersection coincide and represent one point of tangency. The cartesian coordinaltes of this point can be calculated using Equation 4.45.

\subsubsection{Iterative Procedure for Available Sight Distance}

Based on the mathematical formulation presented above, an iterative procedure has been developed to determine the available sight distance on any horizontal or vertical alignment. In this procedure, the sight distance is initially assumed as $S$, and then $S$ is decreased or increased until the sight line becomes tangent to an element representing a sight obstruction. The following steps summarize the procedure:

1. Idealize the sight obstructions to a series of elements as explained previously.

2. Determine the coordinates of the beginning of the sight line.

3. Determine the coordinates of the end of the sight line.

4. Check the intersection between the sight line and the elements which arc used to idealize the sight obstructions. The point of intersection which represents a sight 
obstruction must be within the limits of both the sight line and the element. A point is within the limits of the element, linear or quadratic, if its local coordinate satisfies the condition: $-1 \leq \eta \leq+1$. A point is within the limits of the sight line if the length of the sight line equals the length of the two lines connecting the point and the two extremes of the sight line (the beginning and the end).

5. If the sight line intersects with any element, $S$ is greater than the available sight distance. Decrease $S$ and repeat steps 3 and 4.

6. If the sight line does not intersect with any element, $S$ is less than the available sight distance. Increase $S$ and repeat steps 3 and 4.

7. If the sight line is tangent to any element, $S$ is equal to the available sight distance. End of iterations.

\subsection{Sortware for Marking No-Passing Zones}

\subsection{Description of Developed Software}

The theoretical procedures, presented in Sections 4.1-4.3, have been translated into two computer programs, MARKH and MARKV, written in Microsoft QuickBasic. The two programs can determine the profile of available passing sight distance, and in turn nopassing zones, on two lane highways due to separate horizontal and vertical alignments, respectively.

The software MARKH uses the MUTCD (1995) standards for no-passing zones which are used by the Ministry of Transportation of Ontario (MTO). For horizontal alignments, the sight line of a driver on the inside lane, the lane nearer to the centre(s) 
of the curve(s), is limited by the edge of a theoretical shoulder of 3-m width. The sight line of a driver on the outside lane can cross the right-of-waly and is limited only by any existing lateral obstruction (single or continuous). In both cases, the sight distance is measured along the centerline of the lane. Therefore, for the inside lane, the software determines the available sight distance due to the theoretical $3 \mathrm{~m}$-width shoulder specified by the MUTCD. For the outside lane, the software determines the available sight distance duc to user specified obstructions. In the case of the existence of more than one obstruction, the software will check the available sight distance against each obstruction and determine the minimum available sight distance.

For vertical alignments, although the MUTCD has set the values of both $h_{1}$ and $h_{2}$ as $1.05 \mathrm{~m}$ and has not mentioned using $h_{m \text { m }}$ or $f_{v}$, the software $M A R K V$ adopts these heights as parameters entered by the user. This would provide higher degree of flexibility in determining $S_{a v}$ according to any other specifications, or if any modifications are adopted in the MUTCD specifications. It should also be noted that the adoption of 1.05 $\mathrm{m}$ height for both the driver eye and the object in the MUTCD is set hasically 10 mark the two lanes at the same time and to reduce the amount of work. Such a problem is not experienced in using $M A R K V$.

For both programs, the user specifies the minimum sight distance, $S_{m}$. required $t$ ) be checked. If the available sight distance is Jess than $S_{m}$, each of the (wo programs determines the available sight distance, $S_{a v}$, at every user-specified step, STEP, and for a user-specified accuracy, ACC. Otherwise, the current station will be skipped and the available sight distance at the next station will be checked. 
In addition to $M A R K H$ and $M A R K V$, another computer software, MARKS, has also been developed based on the methodology presented in Section 4.4. The software determines the profile of available sight distance similar to MARKH and MARKV. However, it has a number of additional features which are: (1) it is a single software that can deal with 2-D torizontal or vertical alignments, (2) it can consider variable lane width on horizontal alignment and spline grades on vertical alignments, and (3) it can create the lateral continuous obstruction in horizontal alignments based on the data of the sideslopes. It should be noted that this last feature could not be included in MARKH because of the limitation of constant lateral clearance which is not applicable for MARKS.

\subsubsection{Soffware Verification}

The developed procedures and software were verified by comparing the results obtained by the software with those obtained graphically using numerical examples having different alignments. The parameters specified for the software were as follows: $S_{m}=250 \mathrm{~m}$, $S T E P=20 \mathrm{~m}$. and $A C C=0.1 \mathrm{~m}$. The actual available sight distances were determined by drawing the same curves using Autocad. Then, for horizontal alignments, the available sight distances on the inside lane were determined by drawing sight lines tangent to the theoretical shoulder. On the outside lane, the sight lines were drawn passing through certain obsiructions input to the software. For vertical alignments, the available sight distances were determined graphically considering the existing crest vertical curves and assuming certain locations for the overpasses on sag vertical curves. 
The alignments used in the verification and samples for the input files and the output results are given in the reports published by the Transportition Research Centre. Lakehead University (Hassan et al, 1994a and 1994h). Figure 4.13 shows an example for the horizontal and vertical alignments that were used in the verification. Also, a sample of the results calculated by the computer programs and those measured graphically is shown in Table 4.1. The results obtained by the programs for all cases were in excellent agreement with those obtained graphically. 


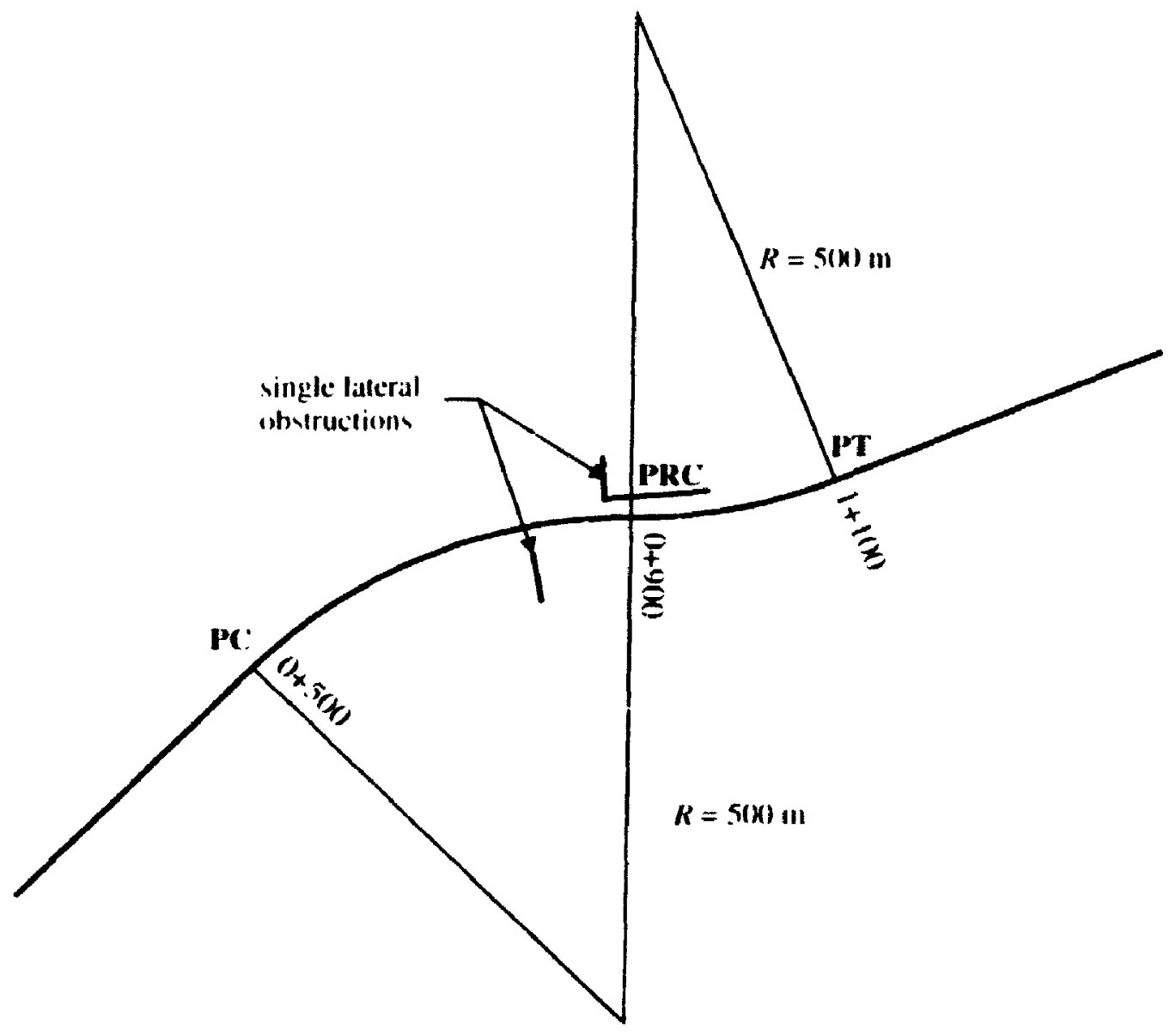

(a) Horrzontal Alignment.

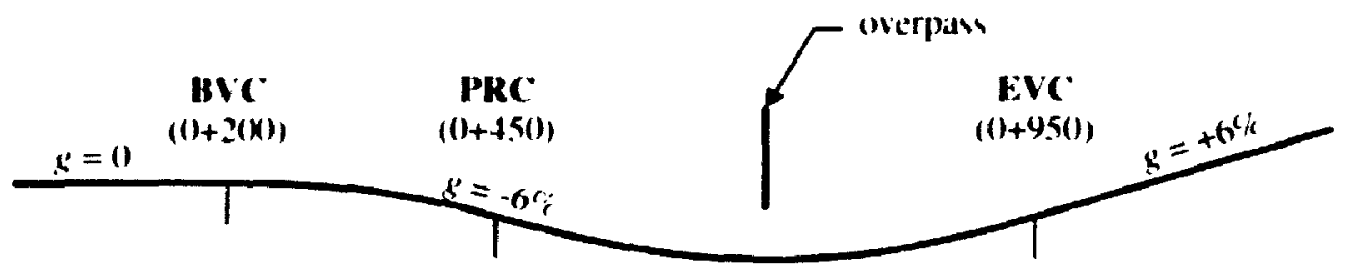

(b) Vertical Alignment.

FIC;URE 4.13: Example of 2-D Verification Alignments. 
TABLE 4.1: Sample of Measured and Calculated Values of 2-1) Available Sight Distance. ${ }^{n}$

\begin{tabular}{|c|c|c|c|c|c|c|c|}
\hline \multicolumn{5}{|c|}{ 2-D Horizontal Alignment ${ }^{h}$} & \multicolumn{3}{|c|}{ 2-D Verticaal Alignment' } \\
\hline \multirow[t]{2}{*}{ Station } & \multicolumn{2}{|c|}{$S_{a s}(\mathrm{~m})^{d}$} & \multicolumn{2}{|c|}{$S_{a}(m)^{r}$} & \multirow[t]{2}{*}{ Station } & \multicolumn{2}{|c|}{$S_{a 1}(m)$} \\
\hline & Calculated & Measured & Calculated & Measured & & Calculated & Measured \\
\hline $0+100$ & 474.9 & 474.9 & $-\cdots-t^{\prime}$ & -....- & $0+000$ & 332.5 & 332.6 \\
\hline $0+200$ & 376.9 & 376.9 & $\cdots$ & ---- & $0+100$ & 248.7 & 248.8 \\
\hline $0+300$ & 280.6 & 280.6 & $-\ldots$ & -..-- & $0+200$ & 205.3 & 205.4 \\
\hline $0+400$ & 190.5 & 190.5 & 415.5 & 415.5 & $0+300$ & $-\ldots$. & -.... \\
\hline $0+500$ & 138.1 & 138.1 & 323.6 & 323.6 & $0+400$ & 425.4 & 425.5 \\
\hline $0+600$ & 138.1 & 138.1 & 236.1 & 236.1 & $0+500$ & 376.7 & 376.9 \\
\hline $0+700$ & 138.1 & 138.1 & 172.9 & 172.9 & $0+600$ & 469.2 & 469.6 \\
\hline $0+800$ & 258.9 & 258.9 & 286.4 & 286.4 & & & \\
\hline $0+900$ & 181.5 & 181.5 & 213.3 & 213.3 & & & \\
\hline
\end{tabular}

a Sight distance is given on the right lane (relative to a driver travelling in the direction of increasing stations)

" Results of MARKH and MARKS

- Results of MARKV and MARKS

d Case of vertical continuous lateral ubstructions at both sides (lateral clearance = $6.5 \mathrm{~m}$ )

- Case of single lateral obstructions (Figure 4.13)

$f$ Sight distance is $500 \mathrm{~m}$ or more 


\section{CHAPTER 5}

\section{AVAILABLE SIGHT DISTANCE ON 3-D}

\section{COMBINED ALIGNMENTS}

This chapter presents an analytical model for determining the available sight distance on 3-D combined horizontal and vertical alignments (Hassan et al, 1996b). The model is an extension to the 2-D model presented in Section 4.4 and is based on idealizing the highway surface and sight obstructions into a net of finite elements that can model planar, curved, and warped surfaces. Then, the sight line, which is a straight line 'netween the driver's eye and the object, is checked against all the possible sight obstructions. If any element representing a sight obstruction intersects with the sight line, the sight line is obstructed. Otherwise, it is an unobstructed sight line. The references used here are Cook et al (1989) and Zienkiewicz and Tay'or (1991).

\subsection{Elements in 3-D Alignment}

Gene: ally, two approaches can be adopted in idealizing the highway alignment into a net of elements: using a large number of simple and small size (micro) elements or using a small number of more accurate and large size (macro) elements. Although the mathematical modelling involved in the second approach is more complicated, this approach produces better accuracy and may require less overall run-time due to the smaller number of elements. Therefore, this approach has been adopted in the developed 
model. Consequently, the elements that are used to idealize the alignment should be able to represent the geometry of planar, warped, and curved highway segments.

Similar to the 2-D elements, the 3-D elements which can have a relatively complex geometry in the global $X-Y-Z$ coordinate system tan be represented by a number of points with known coordinates (nodes). The coordinates of any point within the element can be interpolated among the nodes using the shape functions as follows:

$$
\begin{aligned}
& x=\sum_{i=1}^{n} N_{1} x_{1} \\
& y=\sum_{i=1}^{n} N_{1} y_{i} \\
& z=\sum_{i=1}^{n} N_{1} z_{1}
\end{aligned}
$$

where

$n=$ total number of element nodes,

$x, y, z=$ global cartesian coordinates of the point.

$x_{1}, y_{1}, z_{t}=$ global cartesian coordinates of node $i$, and

$N_{1}=$ shape function of node $i$.

As explained earlier, because the shape functions in the global cartesian coordinates can be very complex, the original element in the global corordinates (paremt element) can be transformed to a simpler element (transformed or parametric element) in an arbitrary local coordinate system. For example Figure 5.1 shows three parent elements, each has four edges, and the parametric element used for each of them. As 

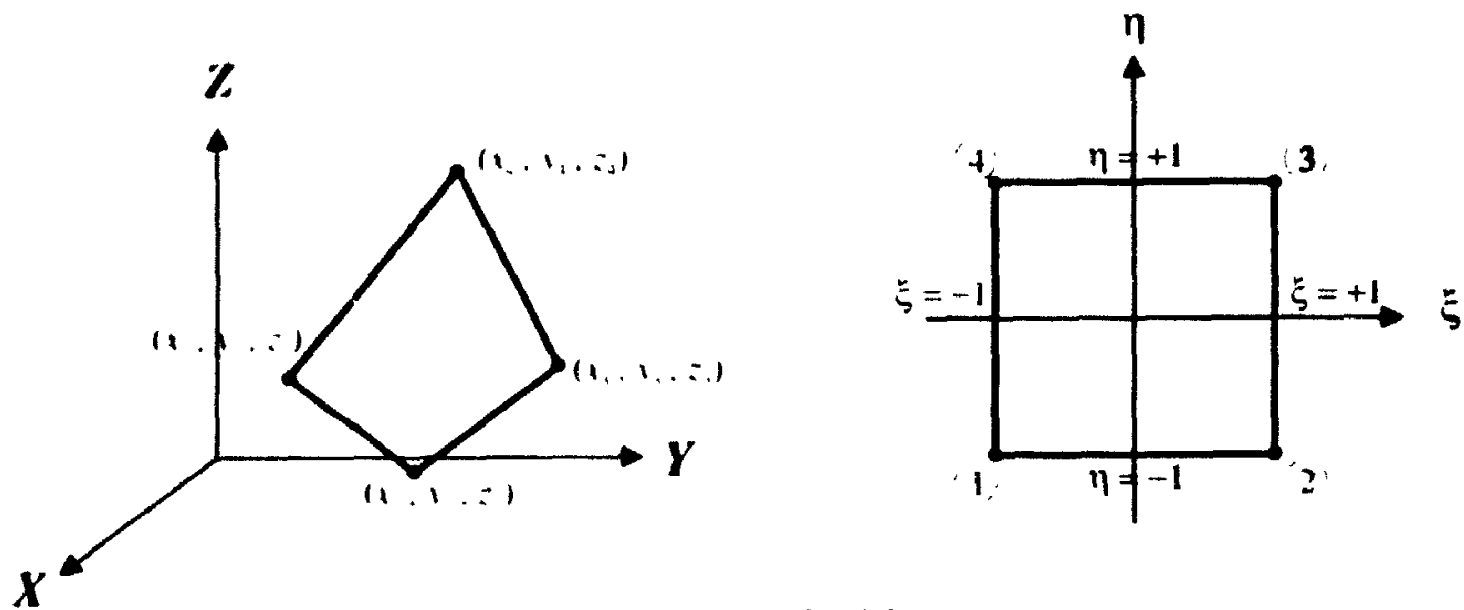

(a) 4-Nide Rectangular Element.
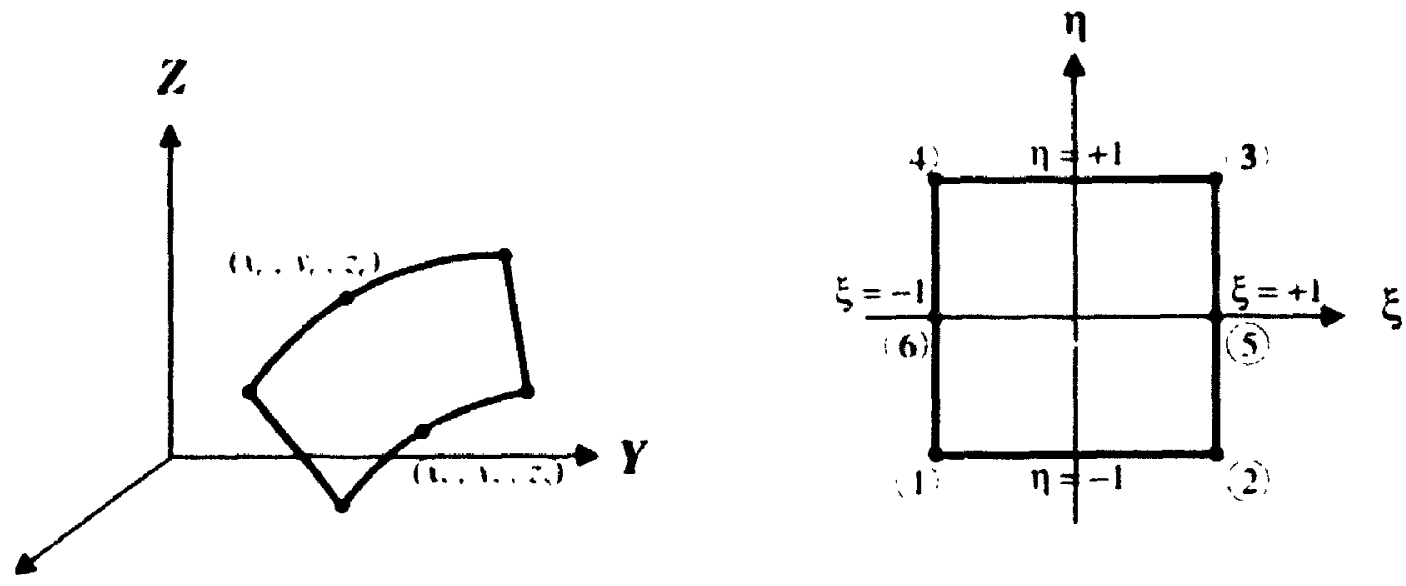

(b) 6-Nis': Rectangular Element.
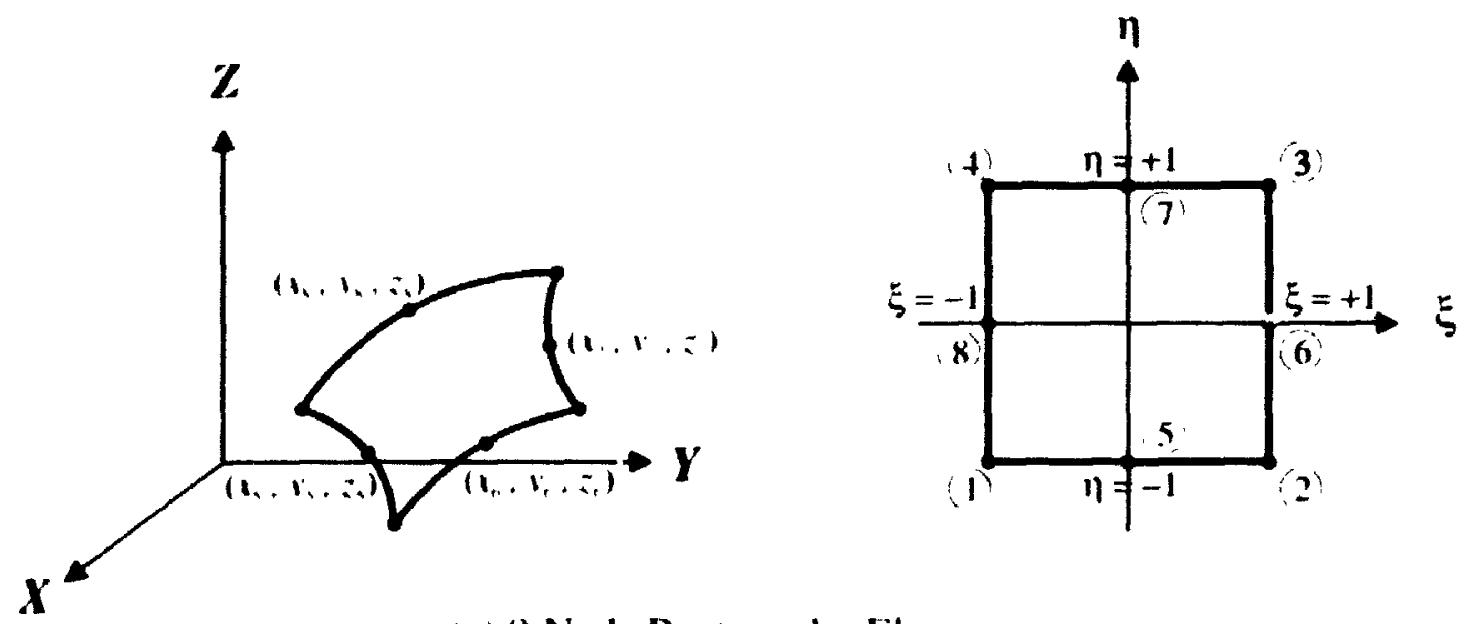

(c) 8-Node Rectangular Element.

FI(jURE 5.1: Rectangular Parametric Elements. 
shown in the figure. the three parametric elements are rectangles in a l(xal $\xi-n$ cinordinalte system.

First. the 4-node rectangular element has a node at each cornor. and thus there are two nodes on each edge. Subsequently. the interpolation along any edge will he linear. Therefore, the interpolation is expected to be exact if and only if all the element's four edges are straight lines, which is the case for highway segnumts which have no vertical or horizontal curvature. However. Jue to the interpolation in two directions, the surface does not have to be planar; instead, it can be warped. Such feature is particularly useful when modelling segments along which the cross-section changes, e.g., straight segments before horizontal curves which are used for the superelevation development.

Second, the 6-node rectangular element has four nodes at its four corners in addition to an extra node at the middle of two parallel edges (parallel to $\eta$-axis). Such an arrangement results is two edges that have three nodes each (parallel to $\eta$-axis) while the remaining two edges have two nodes each (parallel to $\xi$-axis). As a result, the interpolation in the $\eta$-direction is expected to be quadratic (second degrec polynomial) while the interpolation in the $\boldsymbol{\xi}$-direction is still linear. Since a second degrec polynomial (parabola) can be defined by three points. the interpolation in the $\boldsymbol{\eta}$-direction will be exact if the edges of the parent element are parabolic. However, because of the lincar interpolation in the $\xi$-direction, the other two edges of the element must he straight lines. Such surfaces can be experienced on highway segments which have horizontal and/or vertical curvature and have non-curved cross-sections. 
Finally, the 8-node rectangular element is similar to the 6-node element but has an intermediate node on all edges. Following the same argument. the 8-node element can model surfaces that have four curved edges. Such surfaces can be experienced on highway segments which have horizontal and/or vertical curvature and have curved cross-secti is.

In addition to the rectangular elements, triangular elements can also be used. Figure 5.2 shows two types of triangular elements that are transformed into parametric elements in a different local coordinate system. As explained above. since the 3-node element has two nodes along each edge, it can model surfaces with straight edges. However, unlike the 4-node rectangular element, since any three intersecting lines can exist in only one plane, the 3-node triangular element cannot model warped surfaces. As for the 5-node element, it can model surfaces with two curved edges and one straight edge. Similar to the 6-node element, this element can be used on curved segments with non-curved cross-sections. In addition, a 6-node triangular element can be generated from the 5-node element by adding a node at the middle of the third edge. This latter element is similar to the 8-node element and can be used on curved segments with curved cross-sections.

\subsection{Alignment Idealization into Parametric Elements}

The five different elements presented previously can be used in modelling sight obstructions which consist of the highway surface, lateral obstructions, and overpasses. Although all of these elements can be used. selecting an improper element will reduce 


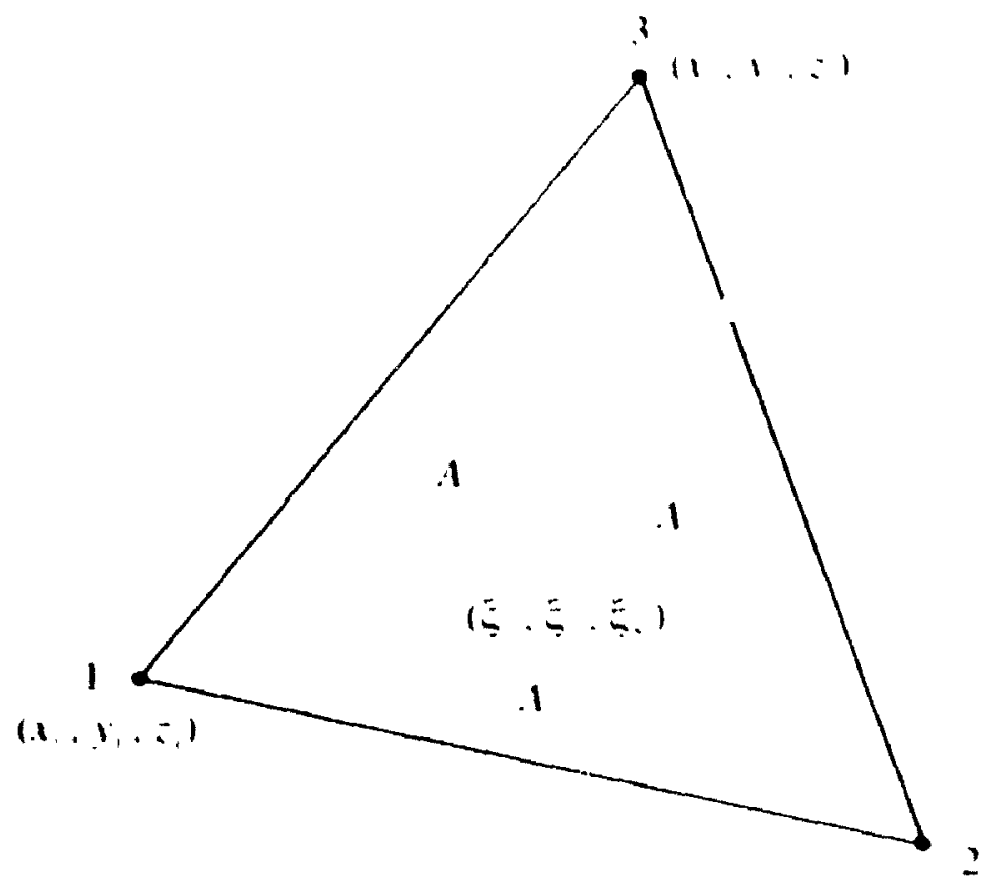

$11,1 \ldots 1$

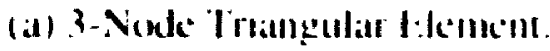

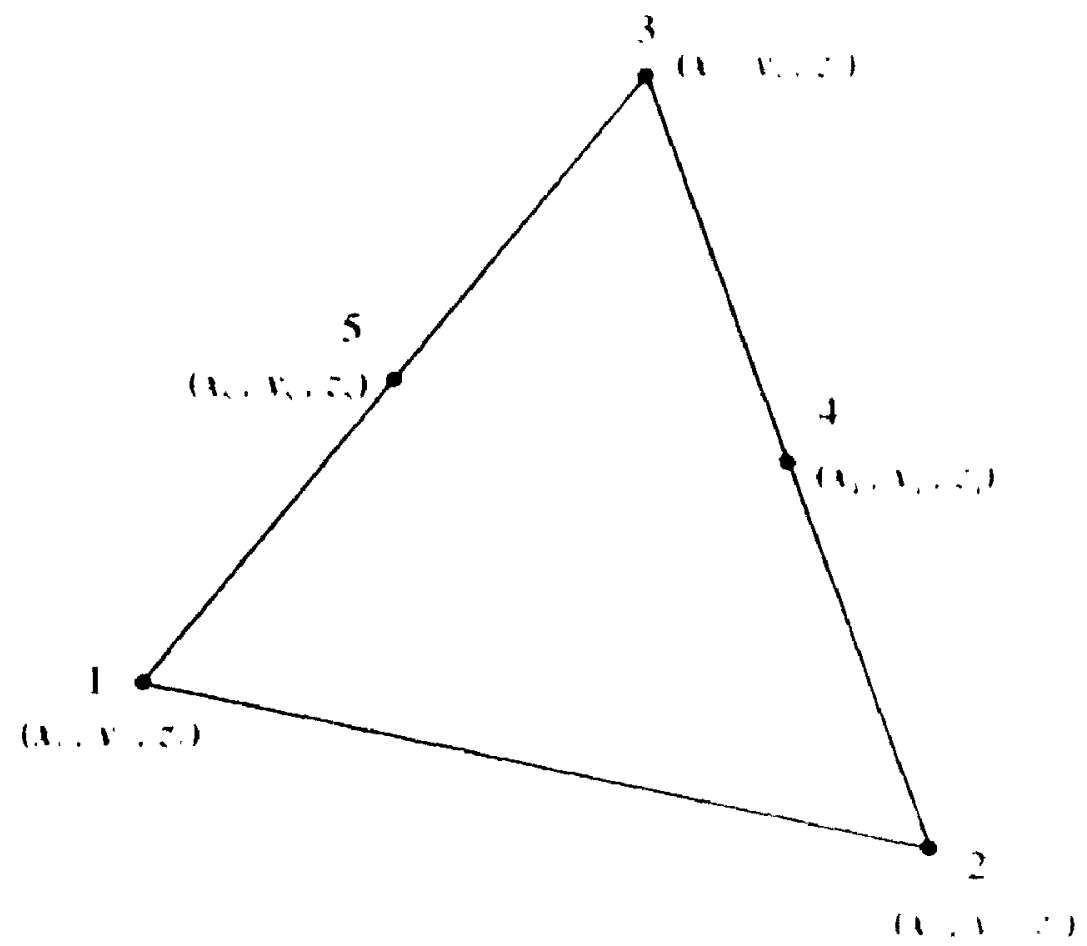

(b) 5-Node Triangulas f:lensent.

FIC;URE 5.2: Triangular Elements. 
the accuracy or increase the complexity of the modelling. Therefore, a good modelling requires a good understanding of the capabilities and limitations of each element.

Surfaces with straight edges can be exactly modelied using the 4-node element where the methudology for the intersection between the sight line and the element is very simple. Therefore, this element can be used to model the highway segments which have no horizontal or vertical curves. Figure 5.3a shows how tangent segments are modelled by the 4-node rectangular elements. As shown in the figure, only one element is required along the whole segment. However, because of the breaks in the segment's cross-section, a number of transversally adjacent elements is required so that every breaking edge will represent the edge of two adjacent elements. Although, the 3-node element has a similar capability, the idealization of the highway into a net of rectangles $w$ ill produce one-half the number of elements required for a net of triangles. Therefore, finding the intersection between the sight line and the rectangular elements will consume almost one-half the time required for triangular elements In addition, the 4-node element can model the warped surface which cannot be modelled by the 3-node element.

As discussed earlier, the 6-node element can model surfaces with two straight edges and two curved edges. Therefore, this element can be used for highway segments with horizontal and/or vertical curves and with non-curved cross-sections. This modelling will be exact if the two curved edges are parabolic, which is the case for curvature due to the vertical alignment. Subsequently, if the curvature is due to a vertical curve only, one element can be used to model the segment regardless of its length. These elements are referred to here as large elements. Figure $5.3 a$ shows also 


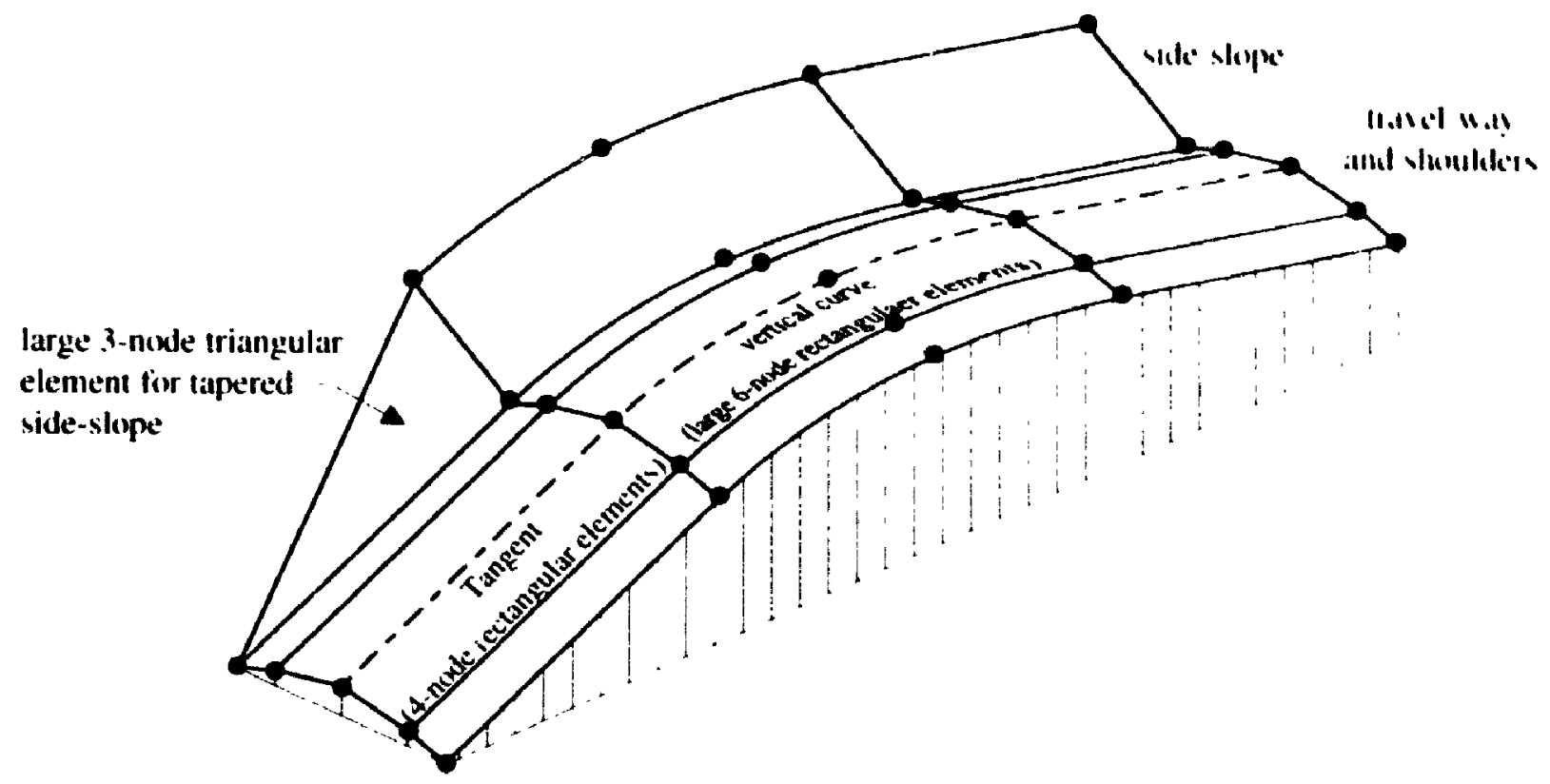

(a) Vertical Curve and Tangent.

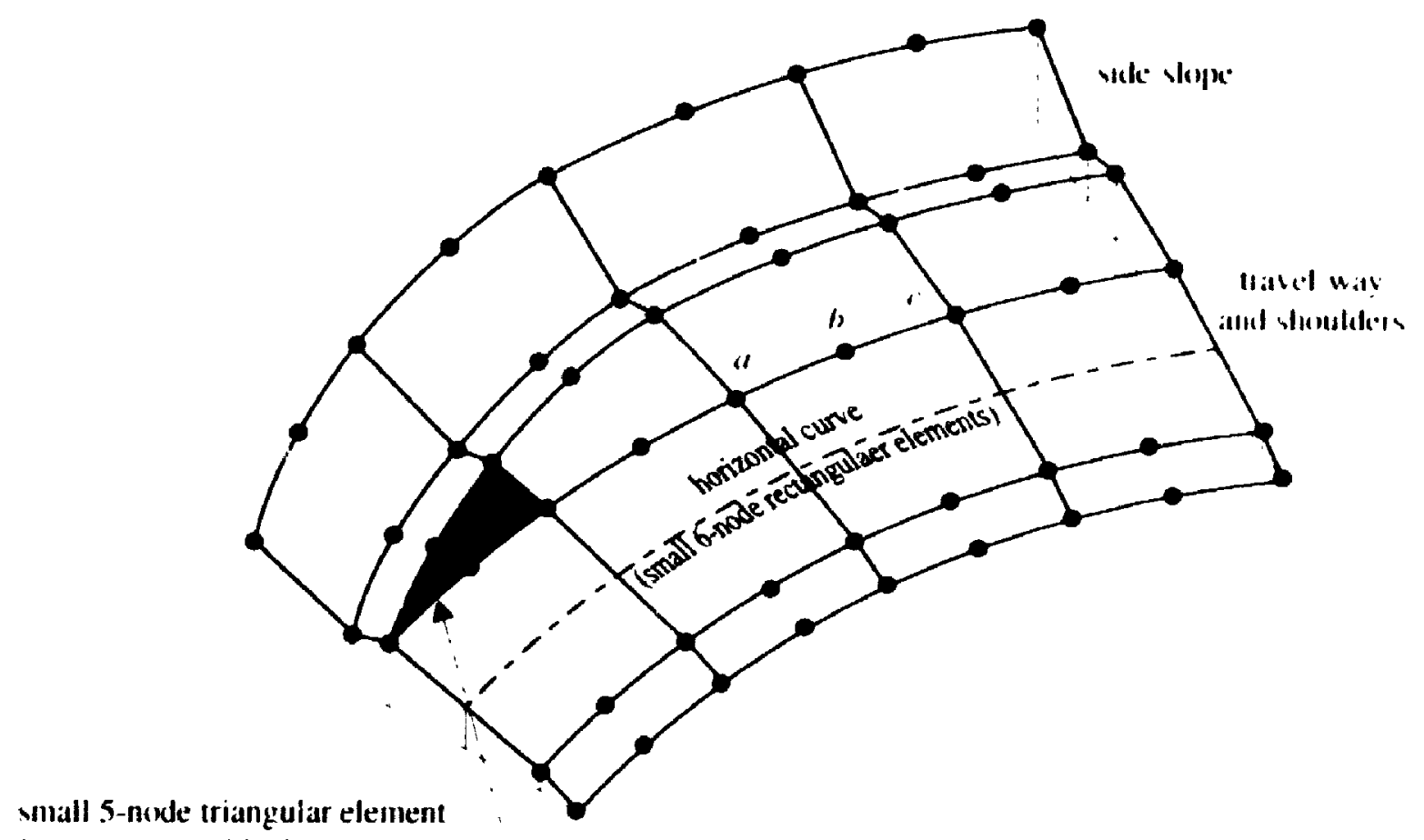
for parement widening on curve

(b) Horisontal Curve.

FICURE 5.3: Idealization of Tangents, Vertical Curves, and Horizontal C Urves into Parametric Elements. 
i 1 example of a crest vertical curve modelled by large 6-node rectungular elements. The elements extend longitudinally from the beginning to the end of the curve. However, similar to the tangent segment, a number of transversally adjacent elements is required so that every breaking edge will represent the edge of two adjacent elements. As shown also in the figure, the continuous lateral obstruction, represented by a side-slope, is also modelled by a 4-node or large 6-node rectangular element if it is on a tangent segment or a vertical curve, respectively. In addition to the parabolic vertical curves, the vertical alignment may include spline grades which do not have explicit mathematical formulas. In this case, small 6-node elements should be used. Generally, the smaller the element size, the greater the modelling accuracy.

On the other hand, since the curvature due to the horizontal alignment is circular or spiral, rather than parabolic, the 6-node element can only provide approximate modelling. The accuracy of this approximation will depend on the element size. Generally, the smaller the element size, the greater the accuracy. Therefore, if the curvature involves a horizontal curve (circular or spiral), the curve should be modelled by a series of 6-node rectangular elements referred to here as small elements. Nonetheless, the element size is still larger than what should be used if the curvature is modelled by planar elements. As shown in Figure 5.3b, a segment on a horizontal curve (or a continuous lateral obstruction on a horizontal curve) is modelled by a number of small 6-node elements where. for example, the circular arc ac is approximated to a parabola (second-degree polynomial) passing through the two ends $a$ and $c$ and the midpoint $b$. However, it should be noted that in using the 6-node rectangular element 
(for vertical or horizontal curves), the cross-section of the highway segment should he linear. If the highway has a parabolic curved cross-section. the 8-node element will be more accurate.

Triangular elements can be beneficial, however, in modelling pavement widening or narrowing when a new lane is added to or removed from the highway. If the extra lane has a cross-slope different from that of the adjacent lane, the widening should be modelled by triangular elements. Triangular elements can also be used in modelling the side-slope, if there is a tapered segment with an increasing (or decreasing) width. As shown in Figure 5.3, a 3-node triangular element should be used if the widening is on a straight segment while a 5-node would be more accurate if the widening is on a horizontal or/and vertical curve with non-curved cross-section. Similar to the rectangular elements, the modelling accuracy will not depend on the element size if it is on a straight segment or a vertical curve, and large elements can be used. If the curvature is due to a horizontal curve, a small element size should he used.

Figure 5.4 shows a highway segment where a part of a horizontal curve coincides with a part of a vertical curve. To idealize this segment into parametric elements, first, 4-node rectangular elements are required for the segment $a b$ because it is a tangent segment in both horizontal and vertical alignments. Second, since segment bc has a vertical curvature only, large 6-node rectangular elements should be used. Then, small 6-node rectangular elements should be used on segment $c d$ (which has horizontal and vertical curvatures) and segment de (which has horizontal curvature only). Irinally, segment $e f$ is modelled by 4-node rectangular elements similar to segment $a b$. If a 


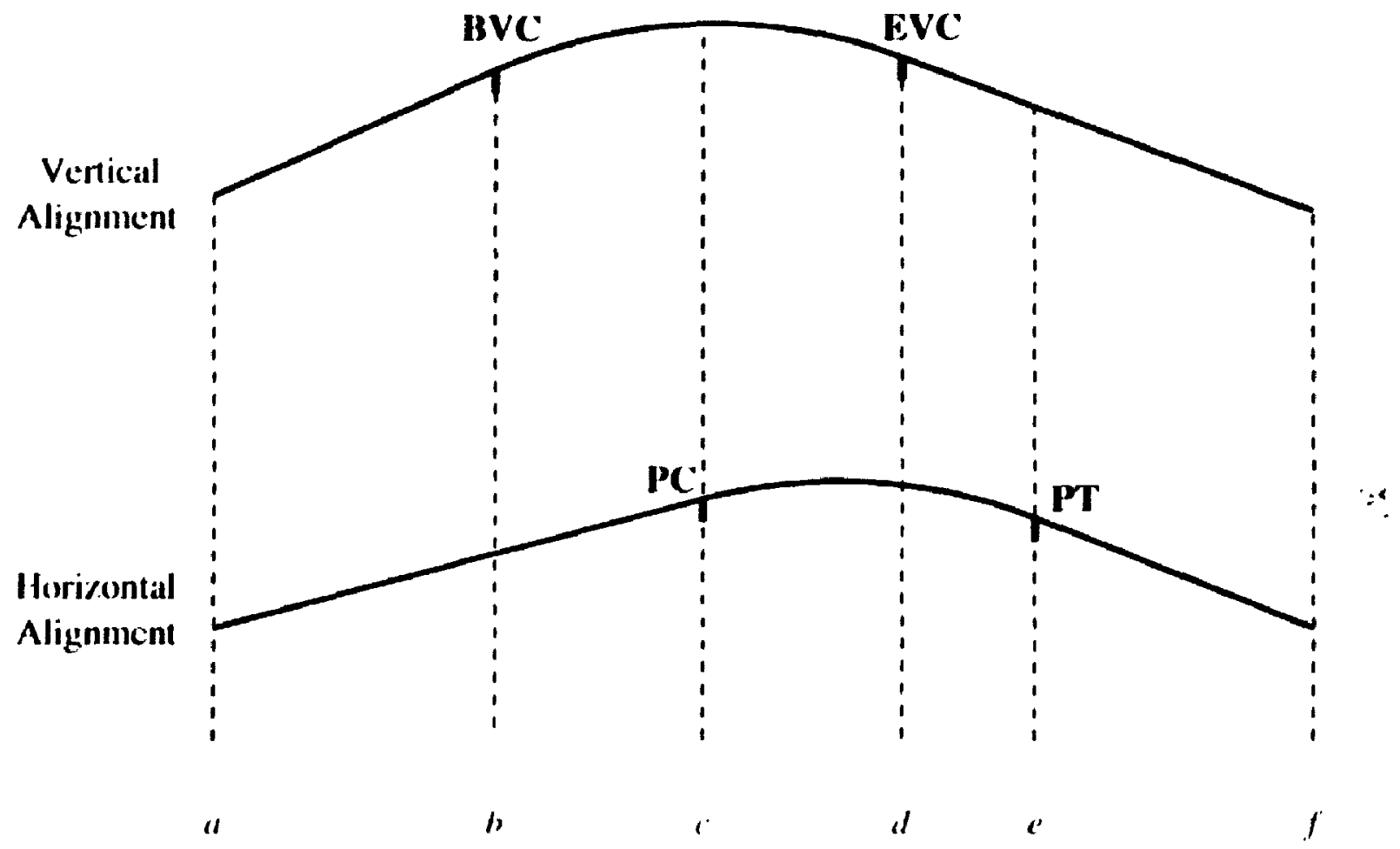

$\mathbf{P C}=$ Point of C'urve

$\mathbf{P r}=$ Point of 'Tangent

BVC $^{\prime}=$ Beginning of Vertical Curve

SVC' = Find of Vertical Curve

FI(iURE 5.4: Idealization of Combined Vertical and Horizuntal Alignment. 
tapered element is encountered on segment $a b$ or ef, a 3-node triangular clement would used. On the other hand, a large 5-node element should be used if the tapered element is on segment $b c$. Finally, a small 5-node element should be used if the tapered element is on segment $c d$ or de.

Single lateral obstructions, which are usually on the inside of a horizontal curve and are represented by a single point in the plan with coordinates $(x, y)$. can be modelled by a 4-node rectangular element. As shown in Figure $5.5 a$, the first edge of this element coincides with the coordinates $(x, y)$ and extends vertically while the element itself extends to the inside of the curve. The element should have vertical and horizontal dimensions large enough to obstruct the sight line if passes beyond the obstruction. In some cases, however, the lateral obstruction cannot be modelled properly using one-point obstruction. Figure $5.6 b$ and $c$ shows schematic representations for other cases where the obstruction cannot be modelled properly with one-point obstruction. In such cases. a number of elements will be required depending on whether the obstruction can be modelled with two or three points.

Similarly, overpasses on sag vertical curves, with a minimum elevation of $z$, can be represented by 4-node rectangular elements. As shown in Figure 5.6a, the first edge of the element will have an elevation of $z$ and extends from the beginning to the end of the overpass. Then, the element extends vertically to an elevation large enough to obstruct the sight line if lies above the overpass. If the overpass is too wide to be modelled by one vertical element, two successive elements can be used instead. The spacing between the two elements will equal the width of the overpass. In audition to 


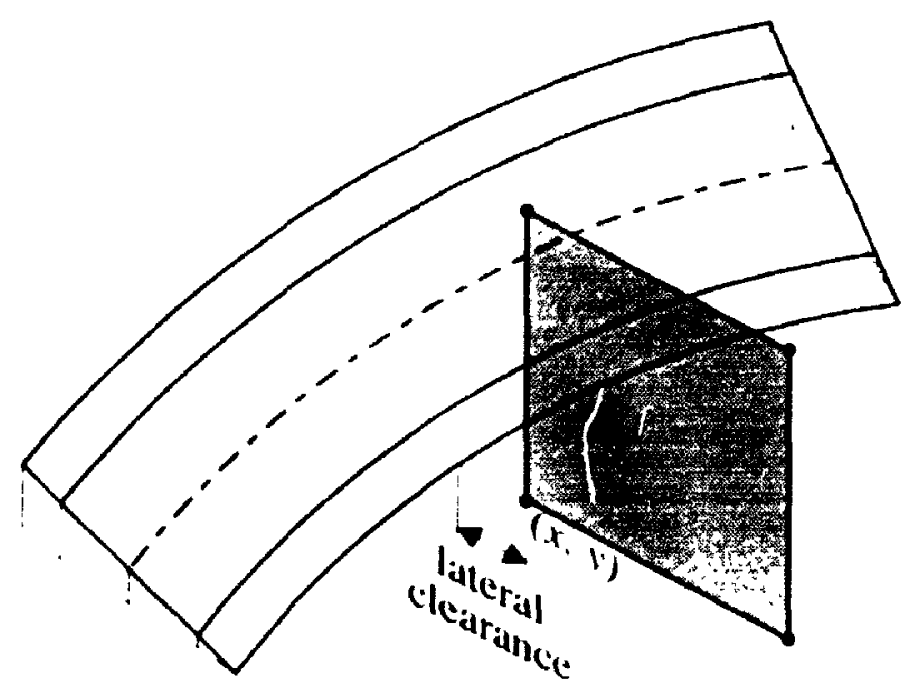

(a) Onc-Point Single Lalteral Obstruction on Horizontal Curve.
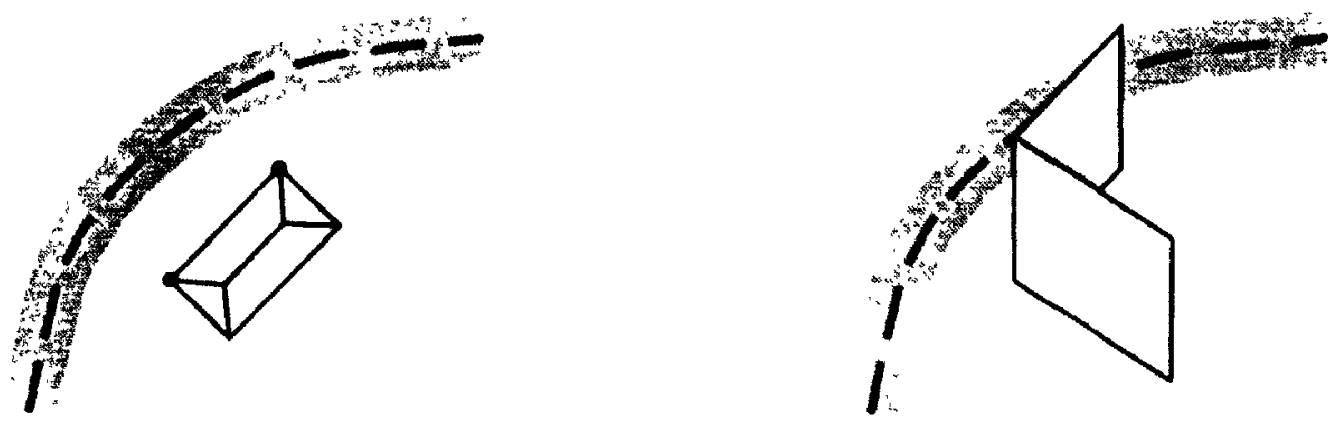

(b) Two-Point Obstruction.
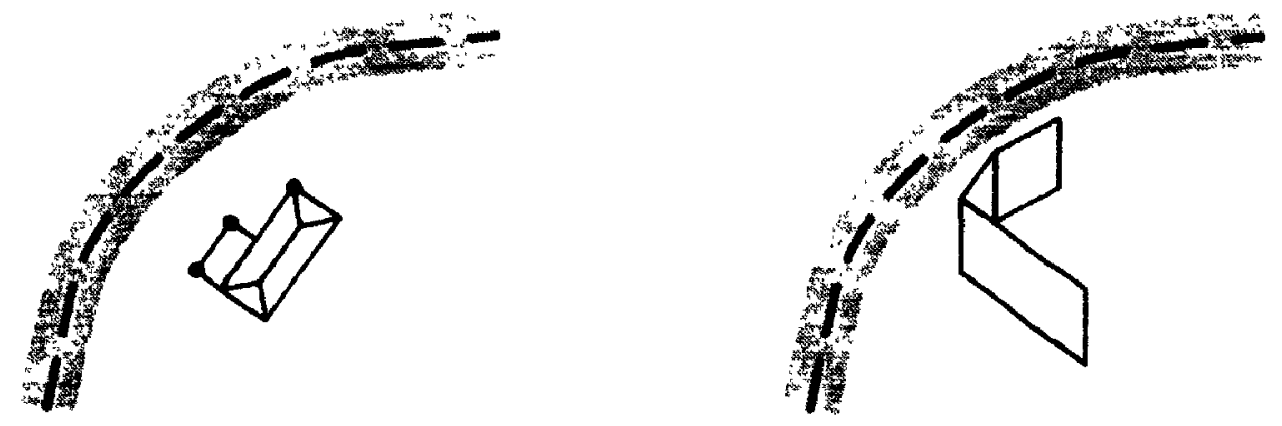

(c) Three-Pont Obsiruction.

FI(;IRE 5.5: Iateral Obstructions and Their Representation by 4-Node Elements. 


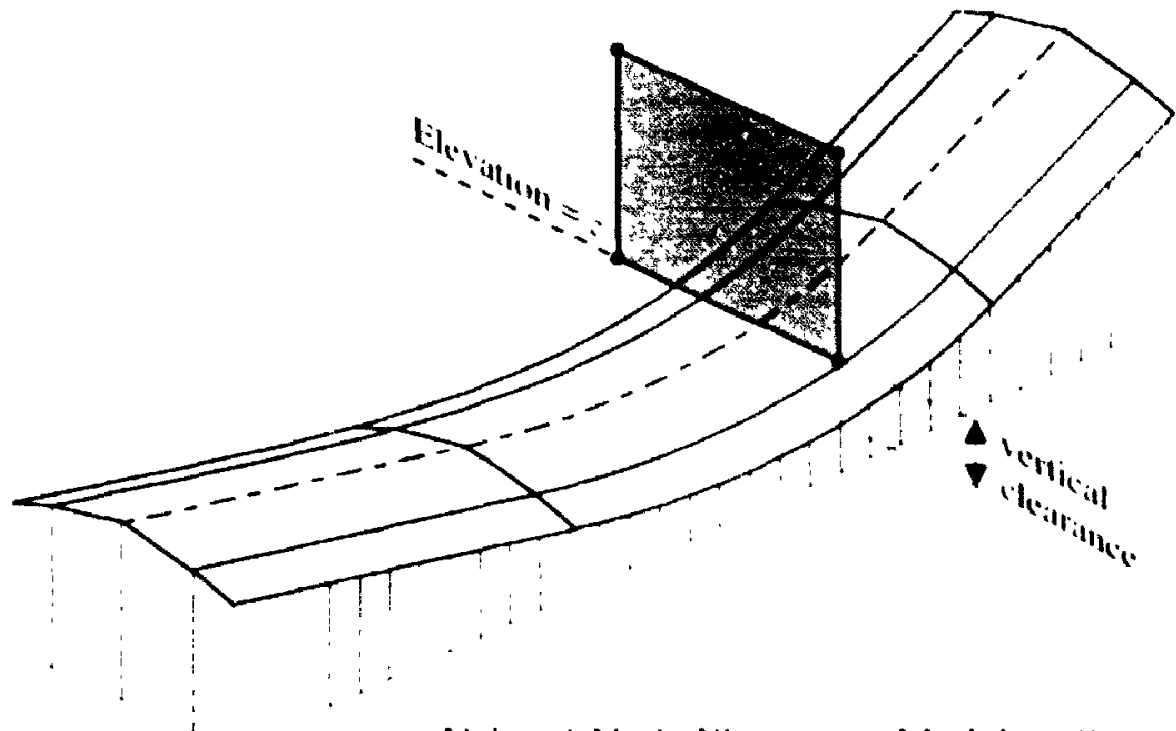

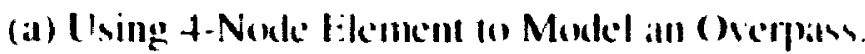

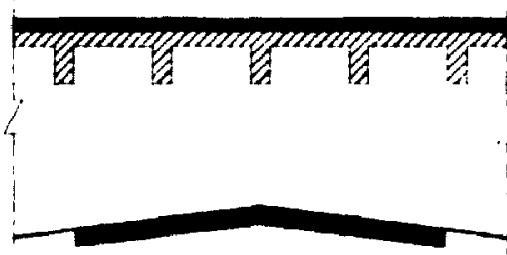

(b) Overpass with Constant Vertical Clearance.
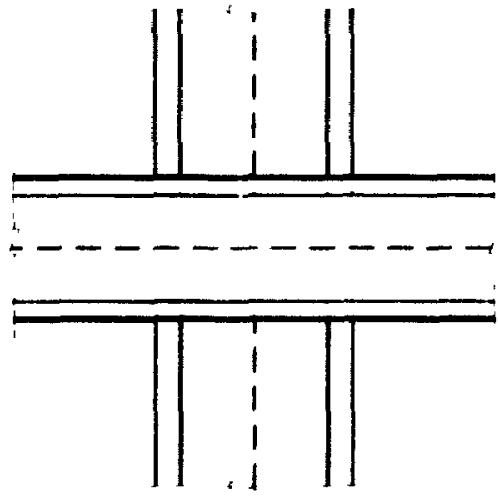

(d) Overpass at Right Angle with the Highway.

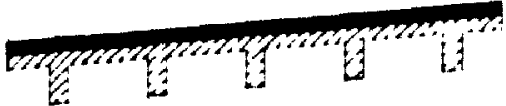

(c) ()erpass will Varratble Vertical cleallance.

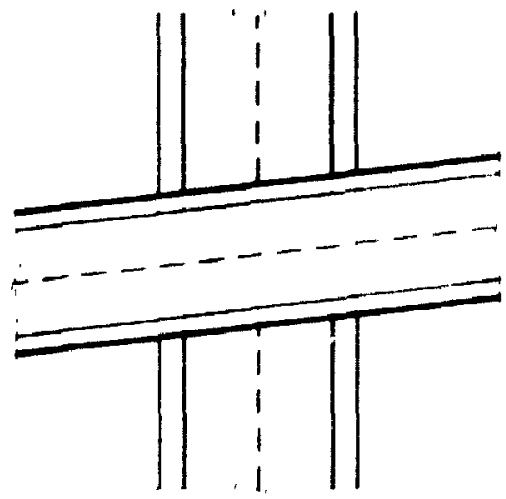

(e) Skewed ()verpas

FIGURE 5.6: Modelling of ()verpasses. 
the overpass width. a proper positioning of the element nodes enables modelling the different types of overpasses, such as overpass with variable vertical clearance or overpasses at skewed angles with the highway (see Figure 5.6 for illustration).

\subsection{Mathematical Modelling}

The mathematical modelling required for the developed iterative procedure is based on clecking the intersection of the sight line, which is a straight line, and the elements used to model the highway alignment. In this modelling, a global system of $X-Y-Z$ coordinates is used to define the 3-D highway alignment. The coordinates $\left(x_{11}, y_{13}, z_{11}\right)$ and $\left(x_{12}, y_{32}\right.$. $\left.z_{s .}\right)$ refer to the cartesian coordinates of any two points on the sight line. Usually, these two points are taken as its beginning and end. Similarly, $\left(x_{1}, y_{1}, z_{t}\right)$ refer to the cartesian coordinates of node $i$. Also, the parameters $a_{1}$ and $b$, refer to constants defined at node $i$ while the parameters $\tau$ and $c$, refer to unique constants in the equations.

\subsection{The 4-Node Rectangular Element}

Referring to Figure 5.1a, the 4-node sectangular element has a node at each of its four corners. The local coordinates are set so that for any point within the element, $-1 \leq \xi$ $\leq+1$ and $-1 \leq \eta \leq+1$. For this local coordinate system and referring to the node numbering system in the figure, the element's shape functions are given as: 


$$
\begin{aligned}
& N_{1}=\frac{1}{4}(1-\eta-\xi+\eta \xi) \\
& N_{2}=\frac{1}{4}(1-\eta+\xi-\eta \xi) \\
& N_{3}=\frac{1}{4}(1+\eta+\xi+\eta \xi) \\
& N_{4}=\frac{1}{4}(1+\eta-\xi-\eta \xi)
\end{aligned}
$$

As discussed in the previous chapter for the linear element, the shape functions of the 4-node element are linear in both $\eta$ and $\xi$ directions. Thus. this elemem! can atcurately model surfaces with straight edges only.

The point(s) of intersection between the sight line and the element can the determined by enhancing the methodology applied in 2-D analysis as follows:

First. the equation of the sight line in 3-D space will be:

$$
\frac{x-x_{s 1}}{x_{32}-x_{s 1}}=\frac{y-y_{31}}{y_{32}-y_{11}}=\frac{z-z_{11}}{z_{12}-z_{11}}
$$

which represents the following two linear equations:

$$
\begin{array}{ll}
x-S_{11} y+\left(S_{11} y_{11}-x_{11}\right)=0 & y_{11} \neq y_{12} \\
x-S_{8 z} z+\left(S_{1 z} z_{11}-x_{11}\right)=0 & z_{11} \neq z_{2}
\end{array}
$$

where

$$
\begin{aligned}
& S_{11}=\left(x_{12}-x_{3}\right) /\left(y_{12}-y_{1}\right) \\
& S_{x z}=\left(x_{52}-x_{3}\right) /\left(z_{32}-z_{1}\right)
\end{aligned}
$$

If $y_{s 1}=y_{s 2}=y_{1}$. Equation 5.4 becomes: 


$$
y-y_{1}-0 \quad\left(y_{1}=y_{2}-y_{1}\right)
$$

Similarly, if $z_{11}=z_{12}=z_{1}$, Equation 5.5 becomes:

$$
z-z_{1}-0 \quad\left(z_{11}=z_{12}=z_{1}\right)
$$

Since the point(s) of intersection hetween the sight line and the element must satisfy the equations of hoth, then

$$
\begin{aligned}
& \sum N_{1} a_{1}+\tau_{1}-0 \\
& \sum N_{1} b_{1}+\tau_{z}=0
\end{aligned}
$$

where

$$
\begin{aligned}
& a_{1}=x_{1}-S_{11} y_{1} \\
& \left(v_{s 1} \neq y_{2}\right) \\
& a_{t}=y \\
& \left(v_{11}=y_{2}\right) \\
& b_{1}=x_{1}-S_{1} z \\
& \left(z, 1 \neq z_{12}\right) \\
& b_{1}=z_{1} \\
& \left(z_{11}=z_{1}\right) \\
& \tau_{1}=-x_{14}+S_{11} r_{1} \\
& \left(y_{11} \neq y_{2}\right) \\
& \tau_{1}=-y \\
& \left(y_{11}=y_{3}\right) \\
& \tau_{i}=-x_{11}+S_{1 z} z_{11} \\
& \left(z_{11} \neq z_{12}\right) \\
& \tau_{z}=-\tau_{1} \\
& \left(z_{11}=z_{2}\right)
\end{aligned}
$$

Substituting for the element shape functions. Equations 5.8 and 5.9 can be written in Lerms of $\eta$ and $\xi$ as: 


$$
\begin{aligned}
& c_{1}+c_{2} \eta+c_{2} \xi+c_{4} \eta \xi-0 \\
& c_{5}+c_{n} \eta+c_{7} \xi+c_{x} \eta \xi-0
\end{aligned}
$$

where

$$
\begin{aligned}
& c_{1}=4 r_{1}+a_{1}+a_{2}+a_{3}+a_{4} \\
& c_{2}=-a_{1}-a_{2}+a_{1}+a_{4} \\
& c_{3}=-a_{1}+a_{2}+a_{3}-a_{4} \\
& c_{4}=a_{1}-a_{2}+a_{2}-a_{4}
\end{aligned}
$$

$c_{s}$ to $c_{x}$ are similar to $c_{1}$ to $c_{3}$. respectively. with replacing $a_{1}$ by $b_{1}$ and $\tau_{1}$ by $\tau_{2}$

Equations 5.10 and 5.11 are (wo polynomials in two unknowns $\eta$ and $\xi$, and cant be solved numerically or analytically. Ising the software MAPI.I: V Wo solve fle equations analytically. $\xi$ is given as the rouls of the polynomial:

$$
\left(c_{3} c_{x}-c_{4} c_{1}\right) \xi^{2}+\left(c_{1} c_{x}-c_{2} c_{2}+c_{2} c_{n}-c_{1} c_{1}\right) \xi+\left(c_{1} c_{1}-c_{3}\right)
$$

Then. $\eta$ is as follows:

$$
\eta=-\frac{c_{5}+c_{\xi} \xi}{c_{6}+c_{k} \xi}
$$

This solution can produce:

1. No solution, if the equation is trivial or due to division by fero. The catse represents a sight line parallel to or coincident with the element, and no sight obstruction exists.

2. Two imaginary roots. This case also represents no intersection. 
3. One real roxt, if $c_{7} c_{x}-c_{4} c_{2}=0$. In this case, one point of intersection exists whose cartesian coordinates can be calculated using Equation 5.1. For this case to represent a sight obstruction, the point of interse. tion must be within the limits of the element. This happens only if $-1 \leq \xi \leq+1$ and $-1 \leq \eta \leq+1$.

4. Two real ron!: (different or identical). This case can happen only with distorted elements, and therefore, it is not applicable in this research.

\subsubsection{The' 6-Node Rectangular Element}

As shown in Figure 5.1b, the 6-node element is similar to the 4-node element but with an additional node at the middle of each of any two parallel edges. For the node numbering shown in Figure $5.1 \mathrm{~b}$, the shape functions of this element are:

$$
\begin{aligned}
& N_{1}-\frac{1}{4}\left(-\eta+\eta \xi+\eta^{2}-\eta^{2} \xi\right) \\
& N_{2}-\frac{1}{4}\left(-\eta-\eta \xi+\eta^{2}+\eta^{2} \xi\right) \\
& N_{1}-\frac{1}{4}\left(\eta+\eta \xi+\eta^{2}+\eta^{2} \xi\right) \\
& N_{4}-\frac{1}{4}\left(\eta-\eta \xi+\eta^{2}-\eta^{2} \xi\right) \\
& N_{4}-\frac{1}{2}\left(1+\xi-\eta^{2}-\eta^{2} \xi\right) \\
& N_{0}-\frac{1}{2}\left(1-\xi-\eta^{2}+\eta^{2} \xi\right)
\end{aligned}
$$

The shape functions of the 6-node element shown in Figure $5.1 b$ are quadratic in the $\eta$ direction and linear in the $\xi$-direction. Subsequently, this element can accurately model surfaces with two curved and two straight edges. 
Following the same derivation as in the calse of the f-llode element, tile coordinates of the points of intersection hetween the sight line and the element can the given as the roots of the following two polynomials:

$$
\begin{aligned}
& c_{1}+c_{2} \eta+c_{1} \xi+c_{4} \eta^{2}+c_{4} \eta \xi+c_{n} \eta^{2} \xi-0 \\
& c_{7}+c_{x} \eta+c_{4} \xi+c_{11} \eta^{2}+c_{11} \eta \xi+c_{12} \eta^{2} \xi-0
\end{aligned}
$$

where

$$
\begin{aligned}
& c_{1}=4 r_{1}+2 a_{4}+2 a_{10} \\
& c_{2}=-a_{1}-a_{2}+a_{3}+a_{4} \\
& c_{3}=2 a_{4}-2 a_{n} \\
& c_{4}=a_{1}+a_{2}+a_{2}+a_{4}-2 a_{4}-2 a_{10} \\
& c_{5}=a_{1}-a_{2}+a_{3}-a_{4} \\
& c_{n}=-a_{1}+a_{2}+a_{3}-a_{4}-2 a_{4}+2 a_{10}
\end{aligned}
$$

$c_{,}$to $c_{12}$ are similar to $c_{1}$ to $c_{n}$, respectively, with replacing $a$, by $b_{r}$ and $\tau_{1}$ by $\tau_{!}$

$a_{1}, b_{1}, \tau_{1}$, and $\tau_{2}$ are as given previously.

Using the software MAPLE-V wo solve Equations 515 and 5.16 analycleally, $\eta$ is given as the roots of the polynomial:

$$
\begin{aligned}
& \left(-c_{11} c_{b}+c_{12} c_{4}\right) \eta^{4}+\left(-c_{11} c_{5}-c_{k} c_{1}+c_{11} c_{4}+c_{12} c_{1}\right) \eta^{\prime} \\
& +\left(-c_{10} c_{3}-c_{7} c_{6}-c_{k} c_{6}+c_{11} c_{2}+c_{12} c_{1}+c_{4} c_{4}\right) \eta^{2} \\
& +\left(-c_{1} c_{5}-c_{x} c_{3}+c_{11} c_{1}+c_{11} c_{2}\right) \eta+\left(-c_{1} c_{3}+c_{4} c_{1}\right)-0
\end{aligned}
$$

which can be solved numerically (Goult et al, 1973;. Then, $\xi$ is as follows This solution can produce: 


$$
\xi=-\frac{c_{1}+c_{2} \eta+c_{4} \eta^{2}}{c_{3}+c_{5} \eta+c_{6} \eta^{2}}
$$

1. No solution, if the equation is trivial or due to division by zero. This case represents a sight line parallel to or coincident with the element, and no sight obstruction exists.

2. Imaginary roots only. This case also represents no intersection.

3. One real root. In this case, one point of intersection exists whose cartesian coordinates can be calculated using Equation 5.1. For this case to represent a sight obstruction, the point of intersection must be within the limits of the element. This happens only if $-1 \leq \xi \leq+1$ and $-1 \leq \eta \leq+1$.

4. Two different real roots. In this case, iwo points of intersection exist, and the comments made above are applicable.

5. Two identical real roots. This case represents a point of tangency. No sight obstruction exists.

6. More than Two real roots. This case can happen only with distorted elements, and therefore, it is not applicable in this research.

\subsubsection{The 8-Node Rectangular Element}

As shown in Figure 5.1c, the 8-node element is similar to the 4-node and 6-node elements but with an additional node at the middle of each edge. For the node numbering shown in Figure 5.1c, the shape functions of this element are: 


$$
\begin{aligned}
& N_{1}=\frac{1}{4}\left(-1+\eta \xi+\eta^{2}+\xi^{2}-\eta^{2} \xi-\eta \xi^{2}\right) \\
& N_{2}=\frac{1}{4}\left(-1-\eta \xi+\eta^{2}+\xi^{2}+\eta^{2} \xi-\eta \xi^{2}\right) \\
& N_{3}=\frac{1}{4}\left(-1+\eta \xi+\eta^{2}+\xi^{2}+\eta^{2} \xi+\eta \xi^{2}\right) \\
& N_{4}=\frac{1}{4}\left(-1-\eta \xi+\eta^{2}+\xi^{2}-\eta^{2} \xi+\eta \xi^{2}\right) \\
& N_{5}=\frac{1}{2}\left(1-\xi^{2}-\eta+\eta \xi^{2}\right) \\
& N_{6}=\frac{1}{2}\left(1+\xi^{2}-\eta^{2}-\eta^{2} \xi^{2}\right) \\
& N_{7}=\frac{1}{2}\left(1-\xi^{2}+\eta-\eta^{2}\right) \\
& N_{8}=\frac{1}{2}\left(1-\xi-\eta^{2}+\eta^{2} \xi_{1}\right)
\end{aligned}
$$

The shape functions of the 8 -node element are quadratic in both $\eta$ and $\xi$ direclions. Subsequently, this element can accurately model surfaces with all edges curved.

Following the same derivation as in the case of the 4-nonde and (6-node elements, the coordinates of the points of intersection between the sight line and the clencint call be given as the roots of the following two polynomials:

$$
\begin{aligned}
& c_{1}+c_{2} \eta+c_{3} \xi+c_{4} \eta^{2}+c_{9} \xi^{2}+c_{6} \eta \xi+c_{7} \eta^{2} \xi+c_{k} \eta \xi^{2}=0 \\
& c_{9}+c_{10} \eta+c_{11} \xi+c_{12} \eta^{2}+c_{14} \xi^{2}+c_{14} \eta \xi+c_{15} \eta^{2} \xi+c_{14} \eta \xi^{2}-0
\end{aligned}
$$

where

$$
\begin{aligned}
& c_{1}=4 \tau_{1}-a_{1}-a_{2}-a_{3}-a_{4}+2 a_{5}+2 a_{6}+2 a_{7}+2 a_{x} \\
& c_{2}=-2 a_{4}+2 a_{7}
\end{aligned}
$$




$$
\begin{aligned}
& r_{4}=2 a_{6}-2 a_{\mathrm{k}} \\
& c_{4}=a_{1}+a_{2}+a_{4}+a_{4}-2 a_{6}-2 a_{\mathrm{k}} \\
& c_{3}=a_{1}+a_{2}+a_{3}+a_{4}-2 a_{5}-2 a_{7} \\
& c_{1}=a_{1}-a_{2}+a_{4}-a_{4} \\
& c_{7}=-a_{1}+a_{2}+a_{3}-a_{4}-2 a_{6}+2 a_{x} \\
& c_{x}=-a_{1}-a_{2}+a_{4}+a_{4}+2 a_{5}-2 a_{7}
\end{aligned}
$$

$c_{1}$ to $c_{1,}$ are similar to $c_{1}$ to $c_{4}$, respectively, with replacing $a_{1}$ by $b_{1}$ and $\tau_{1}$ by $\tau_{2}$. $a, b, \tau_{1}$, and $\tau_{2}$ are as given previously.

liquations 5.20 and 5.21 were also solved using the software MAPLE-V to produce the solution in terms of $c_{1}$ to $c_{16}$. However, because the solution is too long and, as explained earlier, this element will not be used, the solution is not given here.

\subsubsection{The 3.Node Triangular Eleme'nt}

As discussed earlier, rectangular elements provide an easier and better modelling of the highway aliznment than triangular elements. However, this element can be particulary importallt in modelling tapered elements on strajght segments. Moreover, the analytical modelling of this element should provide an automated tool for the methodology followed by Sanchez (1994).

Referring to Figure 5.2a. the 3-node clement is a triangle with 3 nodes at its apexes. The main difference between this element and the rectangular elements is the system of local coordinates used in each element. The coordinate system in the triangular clement is the area coordinates $\left(\xi_{1}, \xi_{2}\right.$, and $\left.\xi_{2}\right)$ which can be defined as follows: 


$$
\xi_{1}=\frac{A_{1}}{A} \quad(i=11,3)
$$

where $A$ is the area of the triangle and $A$, are the areas of the subtriangles as shown in Figure 5.2.

The hape functions of this element are simply the area coordinalles. Irom Equation 5.22, it can be proven that:

$$
\xi_{1}+\xi_{2}+\xi_{3}=1
$$

To determine the coordinates of the point(s) of intersection between the sight line and the element, three equations are required. The first two equations call be obtained by substituting for the shape functions in the equations of the sight linc, Ispuations 5.8 and 5.9, as follows:

$$
\begin{aligned}
& \xi_{1} a_{1}+\xi_{2} a_{2}+\xi_{3} a_{3}=-\tau_{1} \\
& \xi_{1} b_{1}+\xi_{2} b_{2}+\xi_{3} b_{3}=-\tau_{2}
\end{aligned}
$$

where $a_{i}, b_{1}, \tau_{1}$, and $\tau_{2}$ are as given previously.

Equations 5.23, 5.24, and 5.25 are three lincar equations in threc unknowns, and can be solved to calculate the area coordinates of the point(s) of intersection. 'Tlie solution of the three equations can produce:

1. No solution, due to singularity. This case represents a sight line parallel to or coincident with the element, and no sight obstruction exists.

2. One real solution. In this case, one point of intersection exists wluse cartesan coordinates can be calculated using Fquation 5.1. For this case to represent a dight 
obstruction, the point of intersection must be within the limits of the element. Referring to Equation 5.22, this happens only if $0 \leq \xi_{1} \leq+1$.

\subsubsection{The 5-Node Triangular Element}

As shown in Figure 5.2b, the 5-node element is a triangle with 3 nodes at its apexes and one node at the middle of two edges. As mentioned earlier, this element can replace the 3-node element if the pavement widening or narrowing occurs on curved sections in horizontal and/or vertical alignments.

For this element, the same area coordinate system used for the 3-rode triangular element is still the best parametric coordinate system. Therefore, Equation 5.23 is still valid. However, the shape functions, for the node numbering shown in Figure 5.2b, will be as follows:

$$
\begin{aligned}
& N_{1}=\xi_{1}\left(1-2 \xi_{3}\right) \\
& N_{2}=\xi_{2}\left(1-2 \xi_{1}\right) \\
& N_{3}=\xi_{1}\left(2 \xi_{3}-1\right) \\
& N_{4}=4 \xi_{2} \xi_{1} \\
& N_{4}=4 \xi_{1} \xi_{1}
\end{aligned}
$$

To determine the coordinates of the point(s) of intersection between the sight line and the element. three equations are required. Equation 5.23 represents the first equation and the other two can be obtained by substituting for the shape functions in the equations of the sight line, Equations 5.8 and 5.9. Solving the three equations using MAPLE-V, $\xi_{1}$ is the roots of: 


$$
c_{0}+c_{1} \xi_{2}+c_{2} \xi_{i}^{2}+c_{1} \xi_{2}^{2}=0
$$

where

$$
\begin{aligned}
c_{0}= & a_{1}\left(-\tau_{2}-b_{2}\right)+a_{2}\left(\tau_{2}+b_{1}\right)+\tau_{1} b_{1} \\
c_{1}= & a_{1}\left(5 b_{2}+b_{3}-4 b_{4} 2 \tau_{2}\right)+a_{2}\left(-5 b_{1}-b_{1}+4 b_{4}-2 \tau_{2}\right)+a_{1}\left(-b_{1}+b_{2}\right) \\
& +a_{1}\left(4 b_{1}+4 \tau_{2}\right)+a_{4}\left(-4 b_{2}+\tau_{2}\right)+\tau_{1}\left(-2 b_{1}+2 b_{2}-4 b_{4}+4 b_{4}\right) \\
c_{2}= & a_{1}\left(-8 b_{2}-4 b_{3}+12 b_{4}\right)+a_{2}\left(8 b_{1}+4 b_{3}-12 b_{4}\right)+a_{1}\left(4 b_{1}-4 b_{2}+4 b_{4}-4 b_{4}\right) \\
& a_{4}\left(-12 b_{1}-4 b_{1}\right)+a_{4}\left(12 b_{2}-16 b_{4}+16 b_{4}\right) \\
c_{3}= & a_{1}\left(4 b_{2}+4 b_{3}-8 b_{4}\right)+a_{2}\left(-4 b_{1}-4 b_{1}+8 b_{5}\right)+a_{3}\left(-4 b_{1}+4 b_{3} \quad 8 b_{4}+8 b_{1}\right) \\
& a_{4}\left(8 b_{1}+8 b_{3}-16 b_{4}\right)+a_{4}\left(-8 b_{2}-8 b_{3}+16 b_{4}\right)
\end{aligned}
$$

Then $\xi_{2}$ and $\xi_{1}$ can be given as follows:

$$
\begin{aligned}
& \xi_{2}=-\frac{\left(-2 b_{1}-2 b_{3}+4 b_{5}\right) \xi_{1}^{2}+\left(3 b_{1}+b_{1}-4 b_{4}\right) \xi_{1}+\left(-b_{1}-\tau_{3}\right)}{\left(-2 b_{1}+2 b_{2}-4 b_{4}+4 b_{4}\right) \xi_{1}+\left(b_{1}-b_{3}\right)} \\
& \xi_{1}=1-\xi_{2}-\xi_{1}
\end{aligned}
$$

This solution can produce:

1. No solution, if Equation 5.27 is trivial or due to division by 2 eto. The case represents a sight line parallel to or coincident with the elememt. and no sphth obstruction exists.

2. One real root (note that a cubic equation must have at keast one rea! root). In llius case, one point of intersection exists whose cartesian coordinates can be catculatted using Equation 5.1. For this case to represent a sight obstruction, the puint of intersection must be within the limits of the element. This happens only if $0-\xi_{1}$ +1 . 
3. Two different real roots (the third root must be real and repeated). In this case, two points of intersection exist, and the comments made above are applicable.

4. Identical real roots. This case represents a point of tangency. No sight obstruction exists.

5. Three different real roots. This case can happen only with distorted elements, and therefore, it is not applicable in this research.

\subsection{Iterative Procedure for Available Sight Distance}

Based on the mathematical formulation presented above, an iterative procedure can be used to determine the available sight distance on 3-D combined horizontal and vertical alignments. In this procedure, the sight distance is initially assumed as $S$, and then $S$ is decreased or increased until the sight line becomes tangent to an element representing a sight obstruction. The following steps summarize the procedure:

1. Idralize the highway surface and the sight obstructions into a series of elements as explained previously. Since, it is the relative positioning, not the absolute positioning, that controls the sight distance, the cartesian coordinates of each node can be referred to any arbitrary point of origin.

2. Position the driver's eye at the station where the available sight distance is required and detcrmine the coordinates of the beginning of the sight line. These coordinates should be calculated relative to the same point of origin.

3. Position the object al a distance $S$ ahead to the driver's eye and determine the coordinates of the end of the sight line relative to the same point of origin. 
4. Check the intersection between the sight lane and the elements which alle used to idealize the highway surface and the sight obstructions. The pount of entersection which represents a sight obstrtiction must be within the limits of both the sight line and the element.

5. If the sight line intersects with any element, $s$ is grealter than the availahle sight distance. Decrease $S$ and repeat steps 3 and 4.

6. If the sight line does not intersect with any element. S is less than the available sight distance. Increase s and repeatl steps 3 and 4.

7. If the sight line is tangent to any element, $S$ is equal to the available sight distallece. End of iterations.

\subsection{Software for Marking No-Passing Zones}

\subsubsection{Description of Developed Solturare}

The theoretical procedure, presented above, has been translatted into a compulce proy,rall,. MARKC, written in Microsoft QuickBasic. The program can detcrmme the protile of available passing sight distance, and in turn ne passing zones, on Iwo lane lig:liway: duc to 3 -D combined horizontal and vertical alignments. Thiv first version of $M A K K^{\circ}$ is based on the 4-node and 6-node rectangular elenients only. The main mputs for MARKC' are the alignment, cross-section data, and sight obstructions and the mann output w the profile of available sight distance on both lanes of the highway. This profile can be ured to determine the passing and no-passing zones in both directions. $\Lambda$ brief explatiallons of 
the input data, data processing, and the output results of MARKC is presented below, and more detailed explanation with numerical examples can be found in Hassan et al (1995e).

Input Data. The input data are entered to MARKC through a data file prepared by the user before running the program. The data file includes description of the highway alignment, cross-section data. and sight obstructions. In addition, similar to the 2-D computer programs, the input file contains user-specified parameters that determine the accuracy required in determining the sight distance. the stations at which the sight distance will be determined, the minimum value of the sight distance to be checked, $S_{m}$. and the lecights of the driver eye and the object.

The horizontal alignment of the highway centerline is input as a series of straight segments (tangents) and circular and spiral curves. These segments may be arranged in any order. Thus. MARKC can deal with simple and complex alignments. The information required to define the alignment are the station of every point between two successive clements and the turning direction and the radius of each curve. If the element is a spiral curve, the radii al the beginning and end should be identified. Thus. MARKC ciall consider spiral curves connecting two circular curves.

Similarly. the vertical alignment of the highway centerline is input as a series of straight segments (slopes) and crest and sag parabolic curves. The segments mas be arranged in ans order. In addition. the user can identify certain segments as spline grades rather than parabolic vertical curves. Although, spline grades are not used in the design of new highways, ihis feature allows the application of MARKC to existing highways that 
have such type of alignment. The spline grades are modelled by small o-mode clements similar to the horizontal curves.

The cross-section data include the widh and cross-slope of eath of the awo tratrellanes, width and cross-slope of each shoulder, and the side-slope ditla (w hether in a cul or fill section). A total of four segments can be centered on eath side of the highway, and each segment is defined by width and slope (horizontal-to-vertical). If the side-slepe is positive, it will indicate a cut-section, and will be considered ats a comtinuous lateral sight obstruction. A zero value for the slope indicates a cut section with vertical side-slopxe, and will be interpreted as a contintous lateral sight obstruction. On the ofher hand, a negative value for the slope indicates a fill section. Since horiesntal-to-verticall slope cannot define flat segments, a very flat slope will have lo be used (for example, l: $((K K))$. Generally, al set of cross-section data should be entered to the sofiware att any station with a change of any of these data. Widths and slopes at intermediate stations alte extimialled by linear interpolation between the !wo bounding points.

Finally, in addition to the contenuous sight obstructions represconted by the cut slopes, the user can identify the existence of single lateral obstructions and overpassus which can obstruct the sight distance. Single lateral ohstructions (ame-, I wo- - and threepoint) are defined to $M A R K C$ as stations and lateral clearances from the highway centerline. In all cases, the sight line is allowed only to pass within the lateral clearance. If the sight line passes through or beyond the obstruction, it will be considered obstructed. In case of overpasses, the user can specify the station, vertical clearance, and width of each overpass. Skewed overpasses and/or overpasses with vartable vertical clearance can 
also be defined to the software. Similar to lateral obstructions, the sight line must be below the overpass; otherwise, it will be considered obstructed.

Data Processing. The main modules that are incorporated in MARKC are the idealization module and the sight distance module. In the idealization module, MARKC automatically idealizes the alignment and the obstructions into a series of 4-node and 6-node elements according to the concept presented perviously. In the second module. MARKC assumes a relatively low value for the available sight distance $S_{a t}$. Then. $S_{a t}$ is increased gradually with checking the existence of any sight obstruction until the actual $S_{a,}$ is determined at the specified accuracy. However, if $S_{u}$ becomes greater than $S_{m}$, no more iterations are condected al this station. Since $S_{a}$ is determined as the difference between the stations of the driver eye and the object, it will be a distance projected on the highway centerline. Subsequently, a subroutine is added to the software to project this distance on the lane centerline. Therefore, the linai $S_{a 1}$ is a distance projected on the lane centerline similar I1 the MITCD (1995) standards. The module carries on this procedure for all the slations on which the available sight distance is required.

Output Results. The final output is shown first on the computer screen with optıons of sending it to a data file or to a printer. The output contains first a display of the input datti and then the available sight distance on the right and left lane, where the right and left lanes are relative to a driver travelling in the direction of increasing stations. It should be noted also that the available sight distance is given only if it is less than $S_{m}$. 


\subsubsection{Verification}

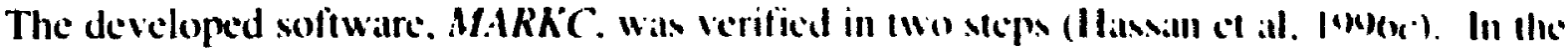
first step, MARKC was verified using a series of sepaltalle horigmtal and lonthal alignments. In preparing the data input, a staight segment was uxed as llev vertical alignment when a horizontal alignment was used for verification. and vice versal. In either case, the cross-section was assumed constant aleng the alignment. The results showed that MARKC can determine the available sight distante on sepaltalte allentuments accuratcly. In the second step, the software was verificel using a combilled allgumbent. Two aiternatives were suggested: to build a physical model alceordmg af all avilumed

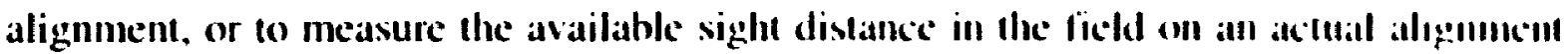
Because the longiludinal and cross-slopes of highwatys are relatlrely sllall. a sltall entol in building the physical model or in measurngl the available sight dostance would magnaly significantly. Therefore. the option of ficld measurements of an actual ally!nument was favoured.

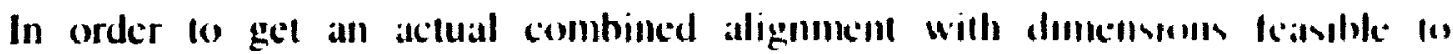
measuring the available sight distance in the feeld and with the sight olstrictions eany lo locate, an overpass was selected, namely the Marina Park ()verpass mil the ('ily of 'Thunde' Bay, Ontario (Figure 5.7). The overpass maps were provided by the ('ity of Thunde' Bay, Engineering Division, and the as-built horizontal and vertical alignun nts alle slown in Figure 5.8. Nonetheless, it was noticed that considerable pavennem selllemilnls had taken place in the fill immediately alter the bridge deck. A lometed levelling wats carnued out to this section to determine the centerline's elevaluon every 5 III Figure 5.9 shows 


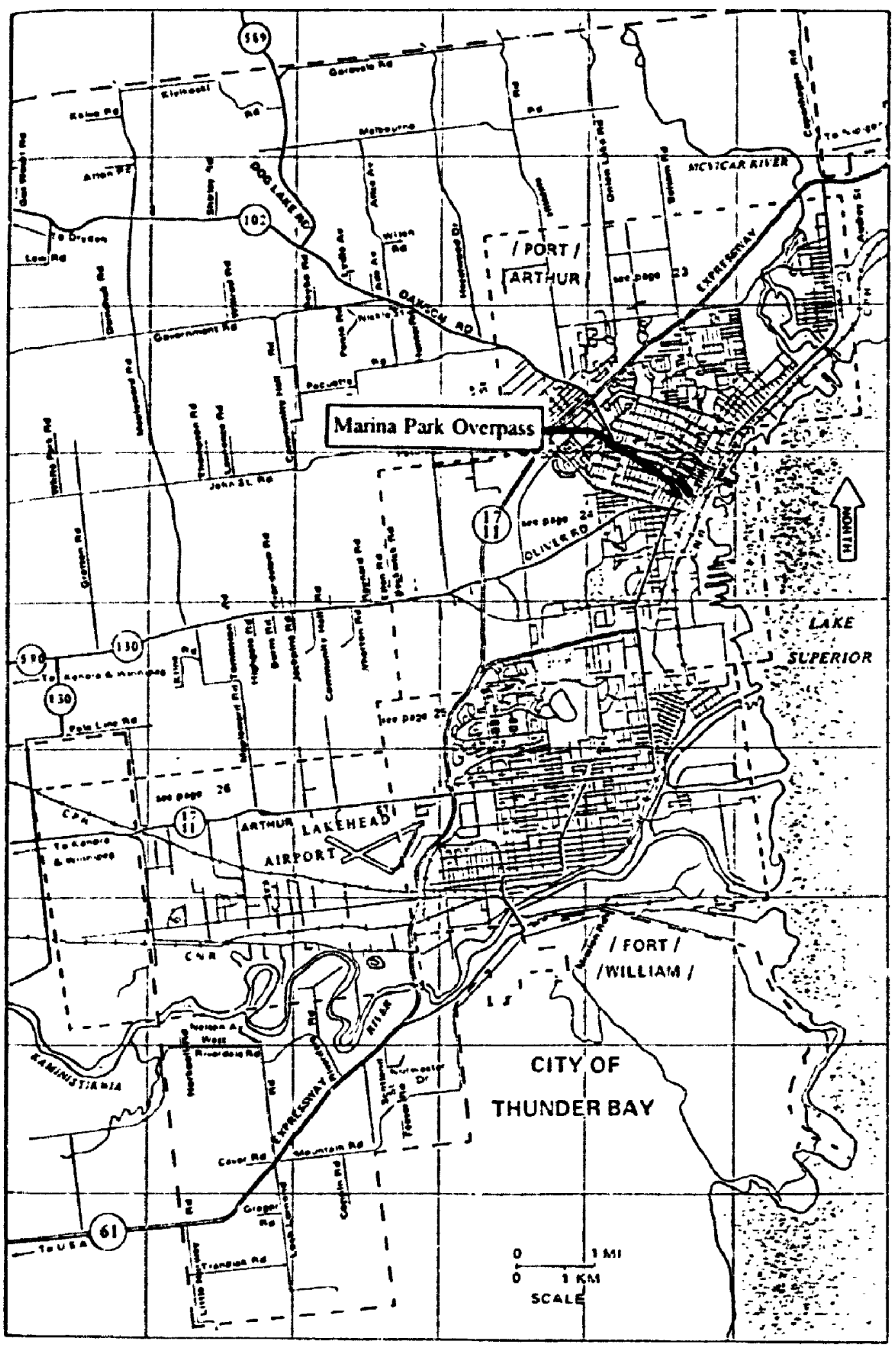

FigURE 5.7: Location of Marina Park Ov' ápass. 


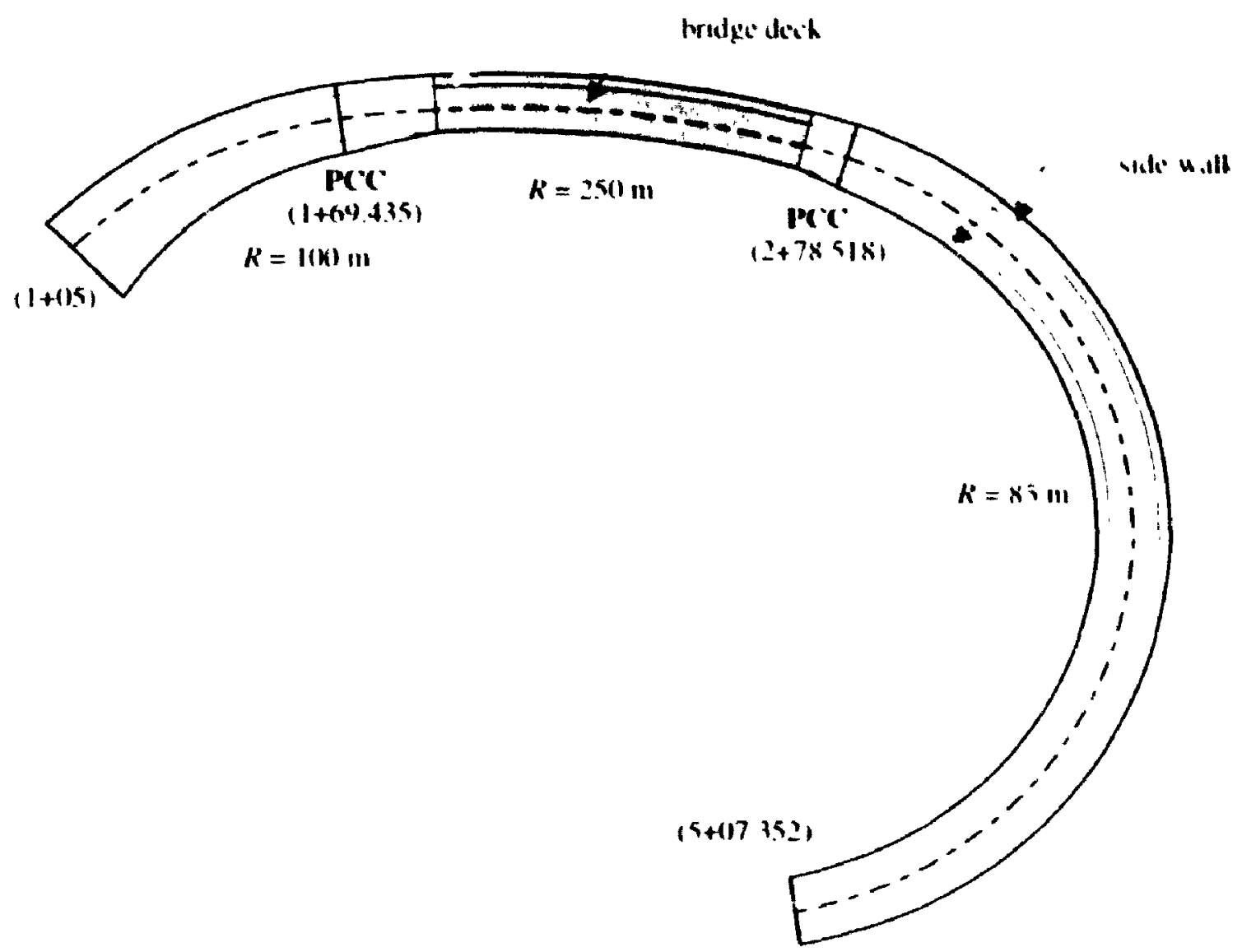

(a) Horizontal Alignment

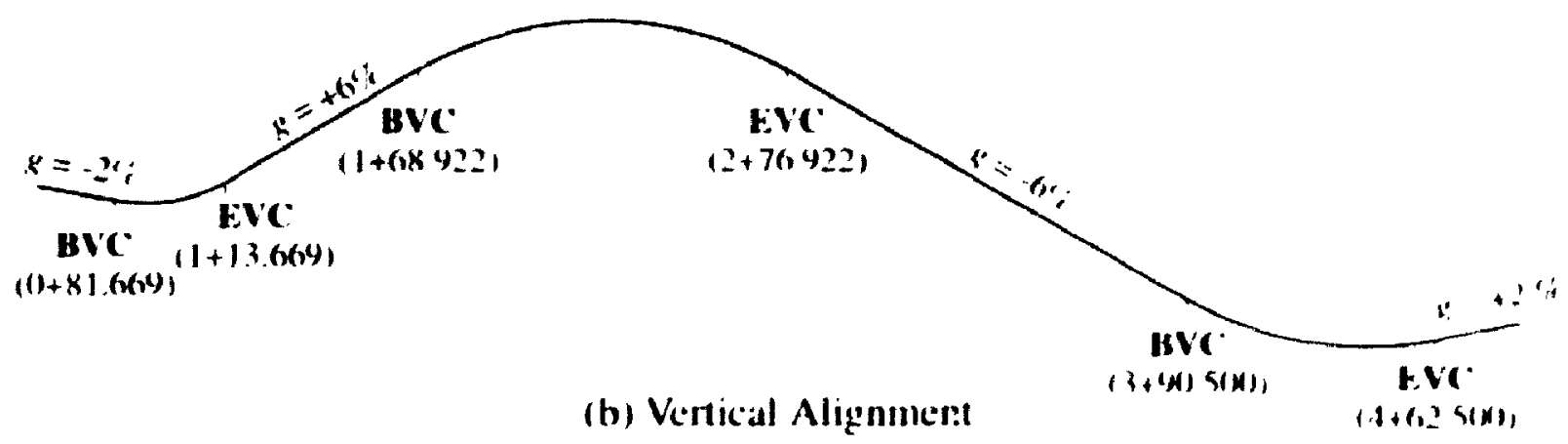

Statwon are every $1(x) \mathrm{m}$.

FIGURE 5.8: As-Built Horizontal and Vertical Alignments of Marina (Bverpass, Thunder Bay, (Ontario. 


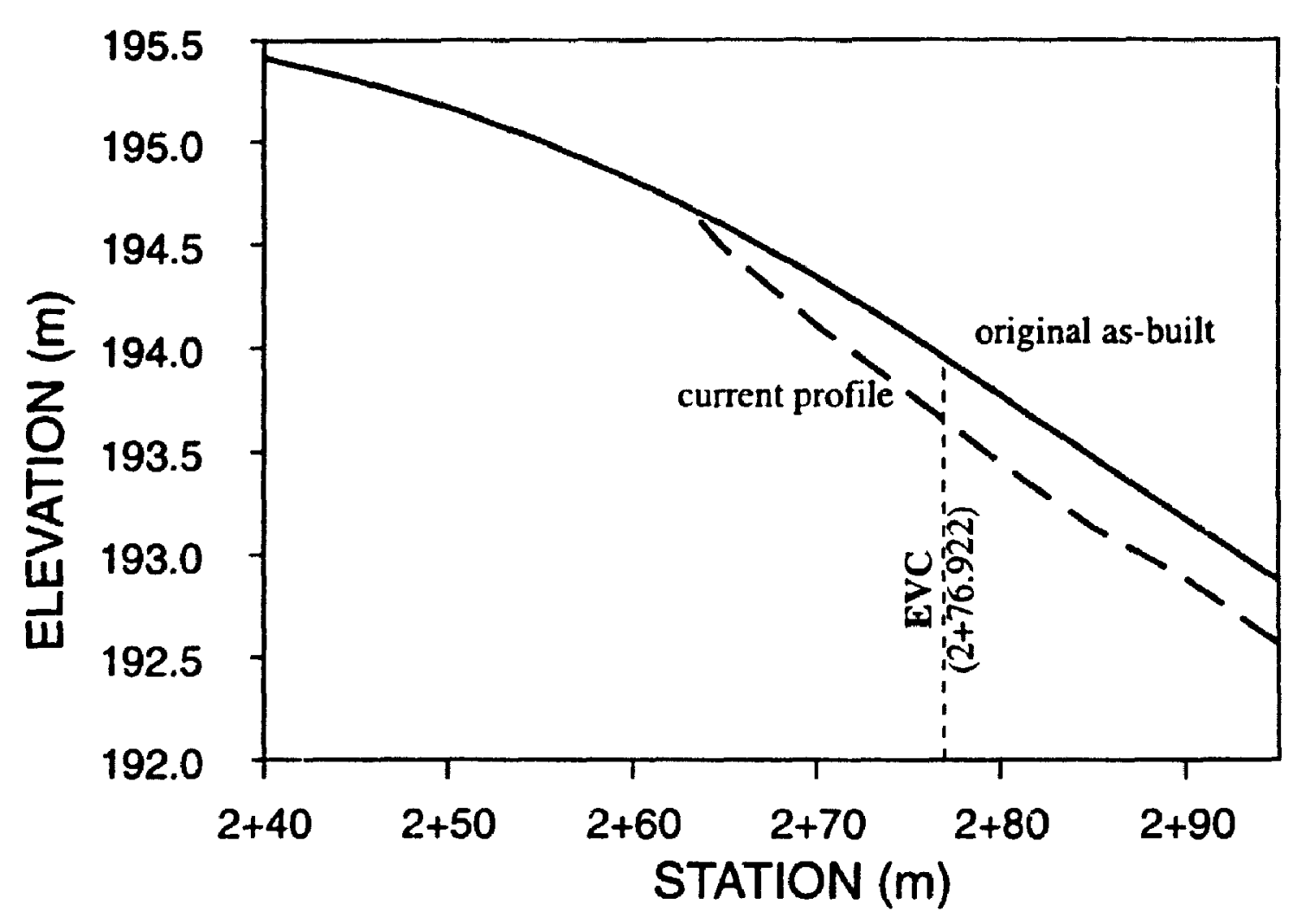

FIGURE 5.9: Original As-Built and Current Vertical Alignments After Bridge Deck.

the centerline's original and current profiles. The cross-section data can be classified into four distinct segments: prior to the bridge deck (variable lane width and lateral clearance), the bridge deck (constant lane width and lateral clearance), immediately after the bridge deck (constant lane width and variable lateral clearance), and the last segment (constant lane width and lateral clearance). Field measurements were carried out to determine the lane width and lateral clearance on the four segments every $10 \mathrm{~m}$. The entire overpass had, however, a constant superelevation rate, namely $4 \%$.

The available sight distance in the ficid was determined using a pair of targets used by the Ministry of Transportation of Ontatio (MTO) in marking passing and no- 
passing zones in the field. As shown in Figure 5.10. cach of the two targets comsists of a rod with two dises, and each disc has an opening. The heights of the iwo openings were measured and found 0.305 and $1.14 \mathrm{~m}$. One of the two targets was used as the driver eye (at the height of $1.14 \mathrm{~m}$ ) and the other was used as the ohject (all the heipht of $0.305 \mathrm{~m}$ ). Based on the advice of the $\mathrm{N}^{\prime} \mathrm{O}$ field crew, side levels were fixed it lese two targets to ensure their vertical setup. Then, the available sight distance on the inside lane was measured every $10 \mathrm{~m}$ beginning at station $(1+0.5)$ and ending at station $(2+8.5)$ for a total of 29 measurements. The alignment data were fed to the program and llev available sight distances at the same stations were determined to the ncitrest $0 .(1) \mathrm{m}$. Setting the maximum element size as $25 \mathrm{~m}$, the element net crealed by $M A R K \mathrm{C}^{\prime}$ to model the overpass is shown in Figure 5.11.

As shown in Table 5.1, the calculated and measured sight dislances were in good agreement for 25 stations where the absolute difference was less than I III (Imaxumum percentage difference was $1.448 \%$ ). However, a considerable error was observed at the stations which had the object immediately after the bridge deck, specially at stations $(2+05)$ and $(2+15)$. Another observation was that the sight distance at these two slations w. re limited by the crest vertical curve in the field while the so!! ware stowed lhat they were limited by the lateral obstruction. The reason for these differences was matnily due to the settlements observed after the bridge deck (see Figure 5.9). This settlement would reduce the effective object height which was estimated as 0.101 and $0 .(140$ m for stallons $(2+05)$ and $(2+15)$, respectively. Therefore, the vertical alignment after the bridge was assumed as a series of broken slopes, as measured in the fieid, and the availatble sight 

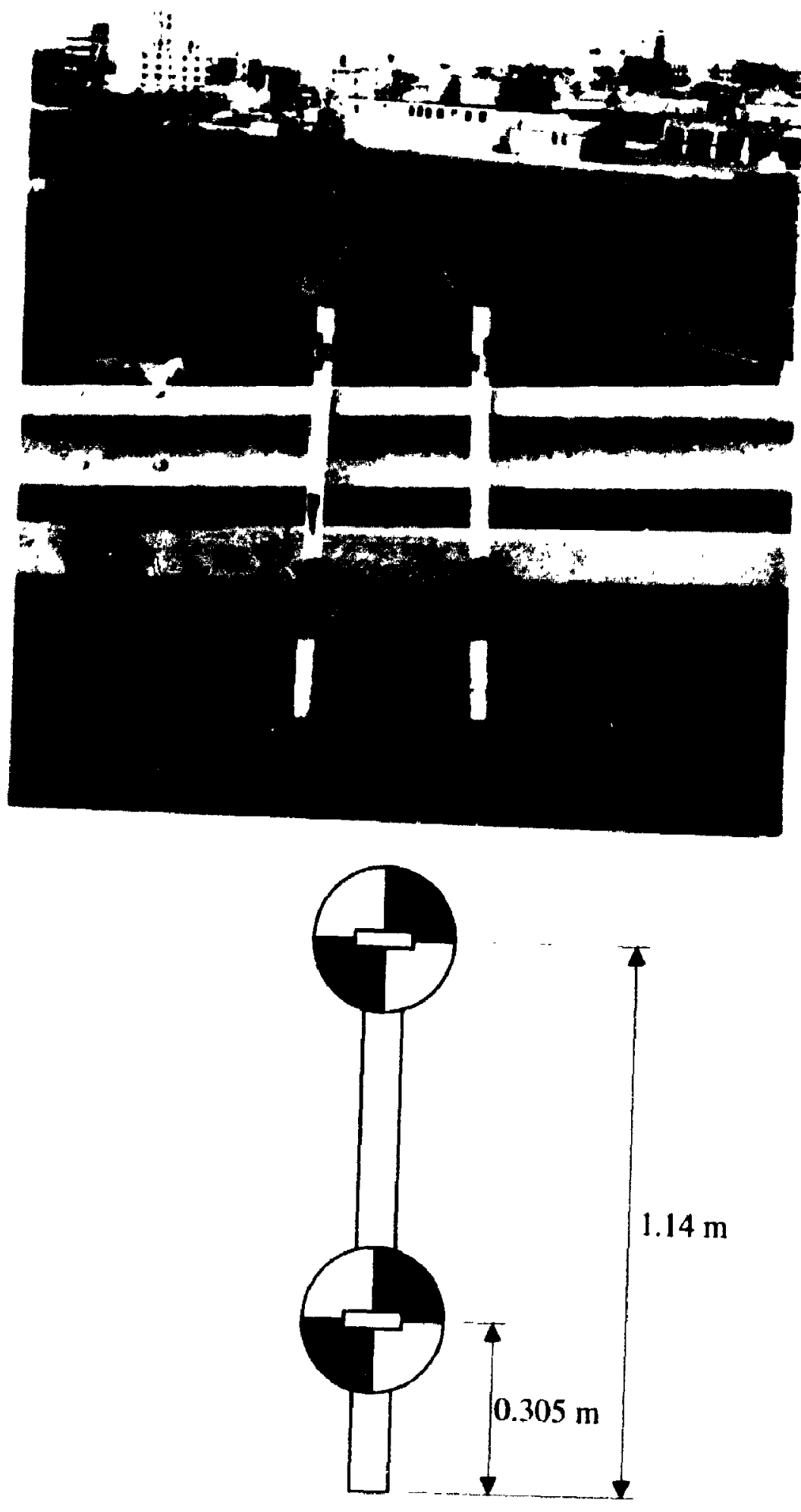

FI(;URE 5.10: Sight Distance Targets Used in Field Measurements. 


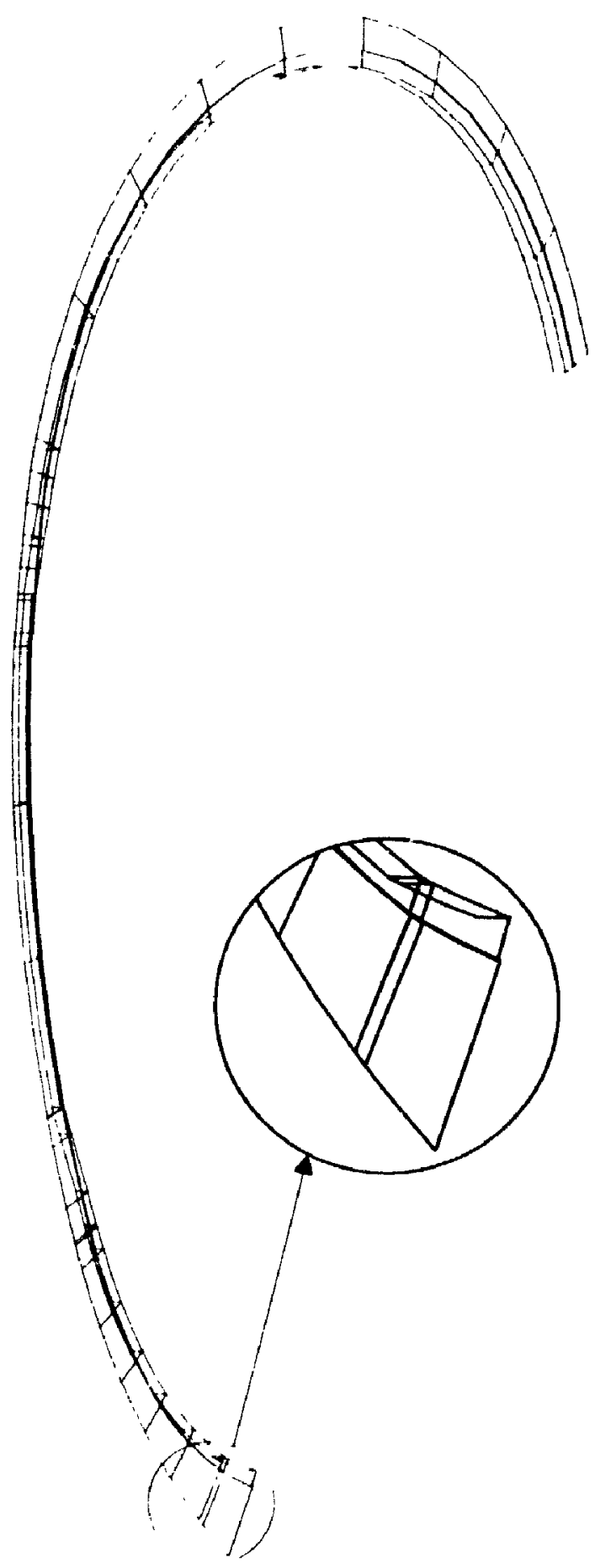

 
TABI.F 5.1: Measured and (alculated Values of the Available Sight Distance.

\begin{tabular}{|c|c|c|c|c|}
\hline \multirow[b]{2}{*}{$\begin{array}{c}\text { Slalion } \\
\text { (min) }\end{array}$} & \multicolumn{2}{|c|}{$S_{a}(m)$} & \multirow{2}{*}{$\begin{array}{c}\text { Absolute } \\
\text { Difterence } \\
\text { (III) }\end{array}$} & \multirow[b]{2}{*}{$\begin{array}{c}\text { Difference } \\
(\dot{\alpha})\end{array}$} \\
\hline & $\begin{array}{l}\text { Meisured } \\
\text { (IFicld) }\end{array}$ & $\begin{array}{l}\text { Calculated } \\
\text { (Soltware) }\end{array}$ & & \\
\hline $1+0.5$ & 60.95 & 60.57 & -0.38 & -0.627 \\
\hline $1+15$ & $0(0.0 \%)$ & 59.47 & -0.62 & -1.043 \\
\hline $1+25$ & 0.3 .102 & 02.06 & 0.36 & -0.575 \\
\hline $1+35$ & 70.11 & 69.97 & -0.14 & -0.200 \\
\hline $1+4.5$ & 0.5 .97 & 66.47 & +0.50 & +0.752 \\
\hline $1+55$ & 07.20 & 67.81 & +0.61 & $+0.90(0)$ \\
\hline $1+0.5$ & 71.94 & 71.48 & -0.46 & -0.644 \\
\hline $1+75$ & 71.42 & 70.91 & $-(0.51$ & -0.719 \\
\hline $1+85$ & 72.06 & 71.12 & -0.94 & -1.322 \\
\hline $1+95$ & 69.50 & 71.12 & +1.62 & +2.278 \\
\hline $2+05$ & 63.74 & 71.12 & +7.38 & +10.377 \\
\hline $2+15$ & 57.70 & 70.08 & +12.38 & +17.666 \\
\hline $2+25$ & 67.53 & 06.81 & -0.72 & -1.078 \\
\hline $2+3.5$ & 01.25 & 02.15 & +0.90 & +1.448 \\
\hline $2+45$ & 59.07 & 58.50 & $-(0.57$ & -0.974 \\
\hline $2+55$ & 59.45 & $0(1.01$ & +0.56 & +0.933 \\
\hline $2+0.5$ & 61.05 & 01.01 & +0.56 & +0.909 \\
\hline $2+75$ & 59.26 & 59.94 & +0.68 & +1.134 \\
\hline $2+85$ & 54.55 & 59.87 & +0.32 & +0.534 \\
\hline $2+95$ & 59.47 & 59.87 & +0.40 & +0.668 \\
\hline $3+0.5$ & $51) .52$ & 59.87 & +0.35 & +0.585 \\
\hline $3+15$ & 54.54 & 59.87 & +0.33 & +0.551 \\
\hline $3+25$ & 50.40 & 59.87 & +0.47 & +0.785 \\
\hline $3+35$ & 50.0 .5 & .59 .87 & +0.22 & $+0 . .367$ \\
\hline $3+45$ & 59.47 & 59.87 & $+(0.40$ & +0.668 \\
\hline $3+5.5$ & 50.55 & 59.87 & +0.32 & +0.543 \\
\hline $3+6.5$ & 00.44 & 59.87 & -0.57 & -0.952 \\
\hline $3+75$ & (1) .27 & 59.87 & $-(0.40$ & -0.668 \\
\hline $3+85$ & 00.35 & 59.87 & -0.48 & $-0.8(02$ \\
\hline
\end{tabular}


distances at these points were recalculated. As shown an Table 5.2. the difference

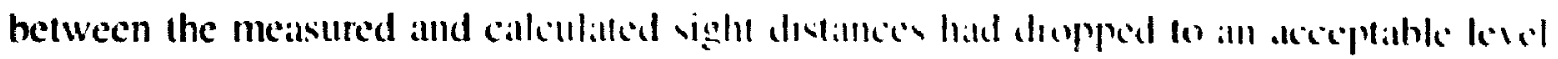

TABLE 5.2: Results of the Modified V'ertical Mlignment.

\begin{tabular}{|c|c|c|c|c|}
\hline \multirow[b]{2}{*}{$\begin{array}{l}\text { Station } \\
(\mathrm{m})\end{array}$} & \multicolumn{2}{|c|}{$s_{\mu}(m)$} & \multirow{2}{*}{$\begin{array}{l}\text { Absolule } \\
\text { Diflerence } \\
\text { (III) }\end{array}$} & \multirow[b]{2}{*}{$\begin{array}{c}\text { Difference } \\
\text { (ai) }\end{array}$} \\
\hline & $\begin{array}{l}\text { Meanured } \\
\text { (Ficld) }\end{array}$ & $\begin{array}{l}\text { (alculitted } \\
\text { (Soltware) }\end{array}$ & & \\
\hline $1+95$ & 69.50 & (6).58 & +0.08 & +0.115 \\
\hline $2+05$ & 6.74 & 6.3 .36 & -0.38 & $-(1) .(1)$ \\
\hline $2+15$ & 57.70 & 57.46 & -0.24 & $-(0)+1 x$ \\
\hline
\end{tabular}

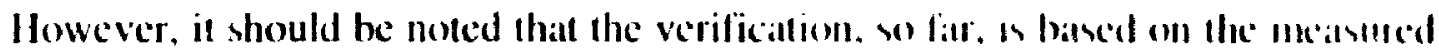

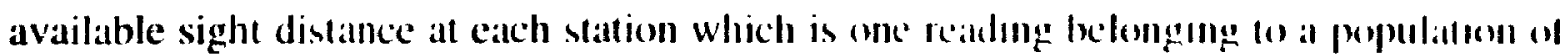
readings. Normally, because the measurement errors are unbiased. seme of the se keadung:4 should be greater than the actual sight distance while the oflhers blumld be lese. However. since the alignment, obstruction. and cross-section on the segmenil after statton $(2+35)$ were the same, the available sight distance sheuld also be the sanne (ther was lle case for

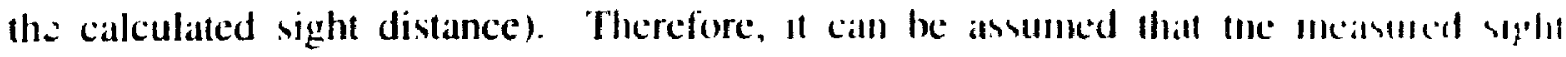
distance at these stations belong to the same population. Beciatuse the number of leadhen, sample size, was small $(n=11)$, it was assumed that they would follew the a distributen The sample's average sight distance, $\bar{x}$, wats $5975 \mathrm{~m}$ and the sannple s standiand de vithnt.

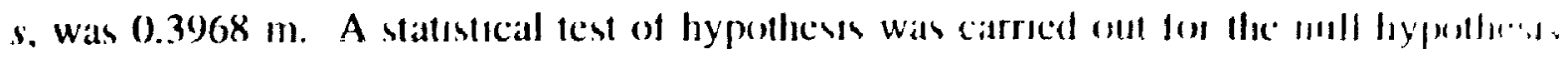
that the actual mean of the sample, $\mu$, was $59.87 \mathrm{~m}$ (at calculated by the solfwalte) and 
the difference between the actual mean and the sample's average was due to the measurement errors. As shown in Table 5.3, the hypothesis is acceptable for a level of signilicance, $\alpha$, of $5 \%$.

TABIE 5.3: Test of Hypothesis for Available Sight Distance.

\begin{tabular}{|c|c|}
\hline 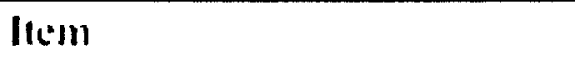 & Value \\
\hline Simple's average, $x$ & $59.75 \mathrm{~m}$ \\
\hline Sample's standard deviation,, & $0.3968 \mathrm{~m}$ \\
\hline Sample sire, $n$ & 11 \\
\hline Degree of freedom, $r=n-1$ & 10 \\
\hline I.evel of symificance, $\alpha$ & $5 \%$ (two-sided test) \\
\hline Null hypothesis, $H_{11}$ & the population mean, $\mu=59.87 \mathrm{~m}$ \\
\hline Calculations & $\begin{array}{l}t_{11}= \pm 2.228 \text { (from the } t \text {-distribution) } \\
t_{1}=\frac{\mu-\bar{x}}{s / \sqrt{n-1}}=0.9563 \\
t_{1}<t_{0}\end{array}$ \\
\hline Conclusion & Accept the hypothesis, $U_{0}$ \\
\hline
\end{tabular}




\section{CHAPTER 6}

\section{UTILIEATION OF DEVELOPED}

\section{MODELS IN MARKING TWO-LANE HIGHWAYS}

In this chapter the models for 2-D and 3-D avialable sight distance, presented in ('hapters 4 and 5, are used to determine the profile of available sight distince ont a teal segentent of a two-lane highway. A discussion about the programs applicability and ancuracy and a comparison between 2-D and 3-D sight distances are presented. Then, lle profiles of 2-D and 3-D available sight distances are used to determine the proper marhing of passingt and no-passing zones according to the MUTCD standards and arcording to the revised mociel developed in Chapter 3. Finally, a comparison among the different methods of marking and the existing marking in the field is presented.

\subsection{Alignment Data}

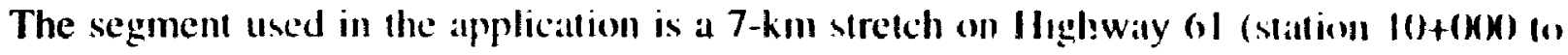
17+000. township of Crooks) between the Canada-lis borders and the ('ily of Illundel Bay (Figure 6.1). The highway is a two-lane lacility with a passing lante addded to dhe right lane (relative to a driver travelling in the dacetuon of increasmg vattums) form station $10+740$ 10 12+550 (including tapers). Another passing lante is asdded to flue Ieft

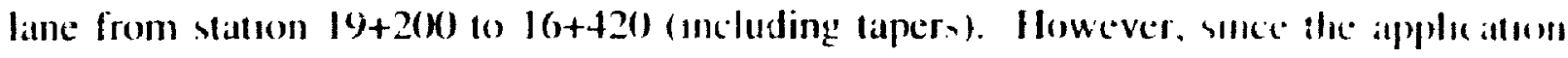

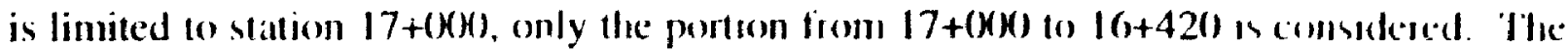
speed limit on Heghway of is $90 \mathrm{kph}$ whle the design speed is $110 \mathrm{kph}$ Accondeng? 11 


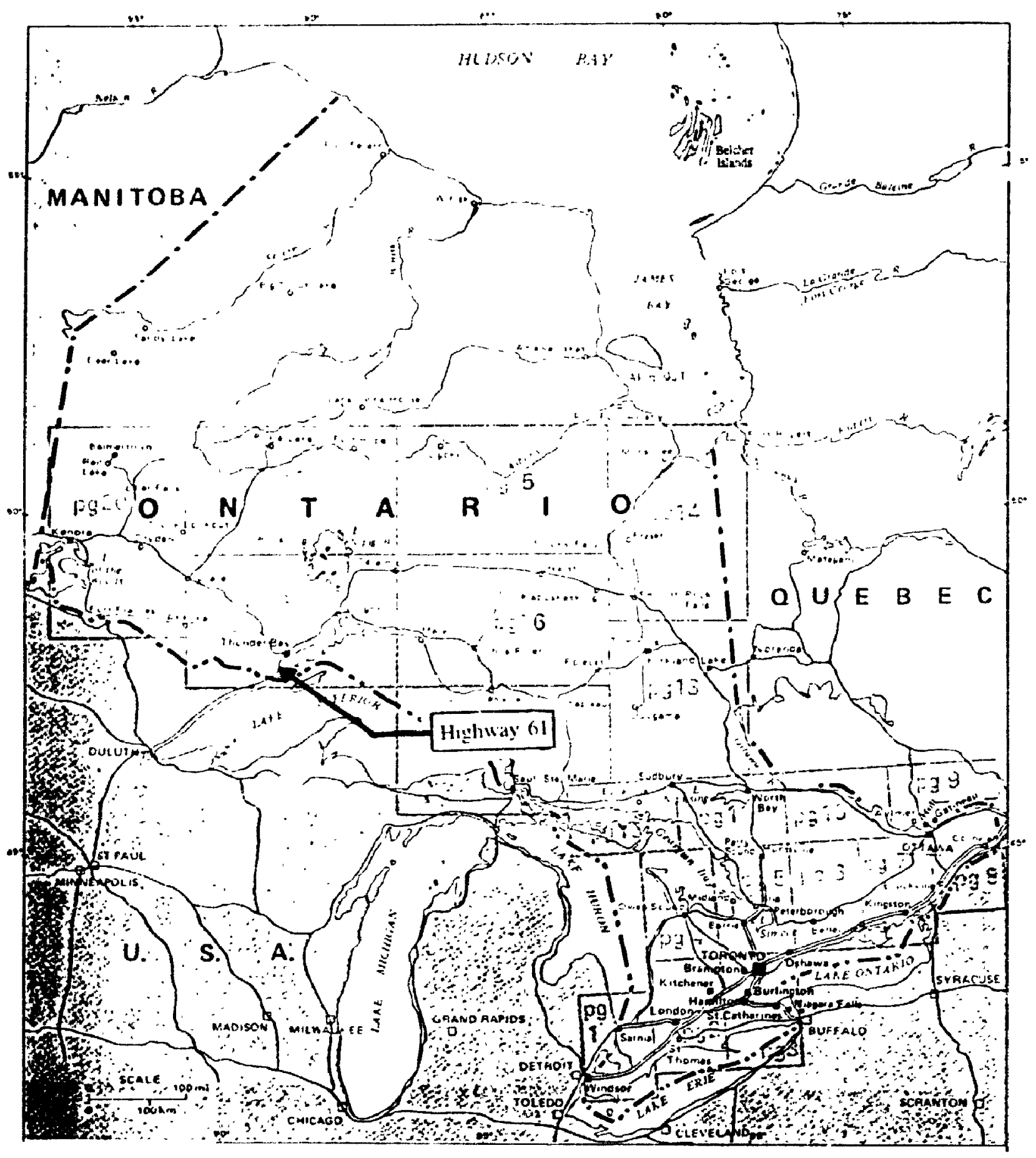

HIf l'RE 6.1: Location of Highway Segment. 


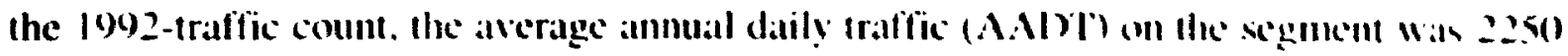

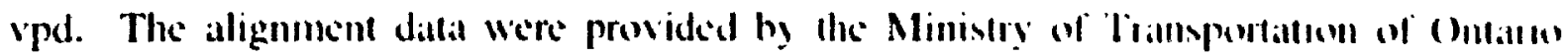
(MTO). Nonetheless, the data of single lateral sight obstructions were men alsalable in

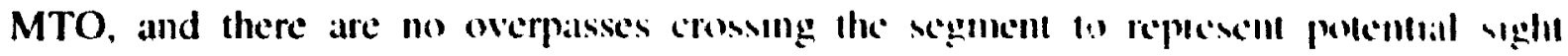
obstructions. Therefore. sight obstructions on the segmemt comsts manty of the hi! hw.ty surface and the cut-slopes in cut sections.

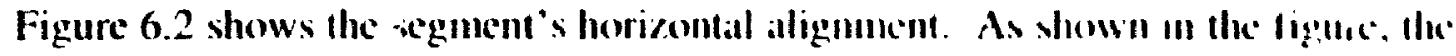
alignment includes a wide variety of horigmtal curves stah as curves with and wallout

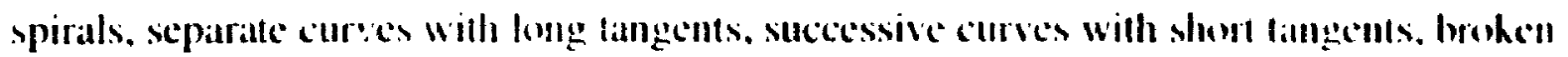

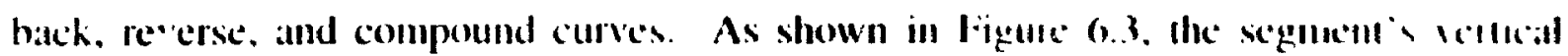

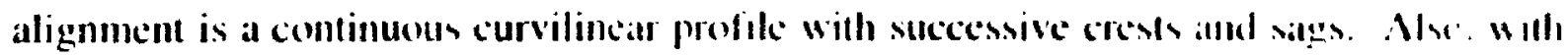

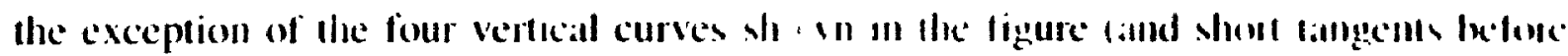
and after each curve), the aignnent consist mainly of stecessive spling prakes. The spline grades are defined by a total of $f(x)$ pomts wh hrown statloms and ele vallums. In

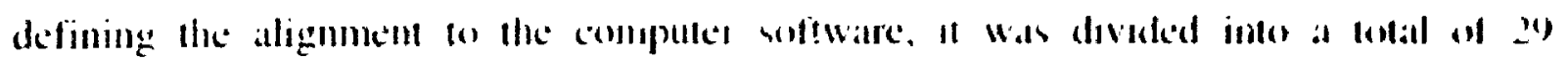
segments 14 curves. 7 tangents, and is splune grates).

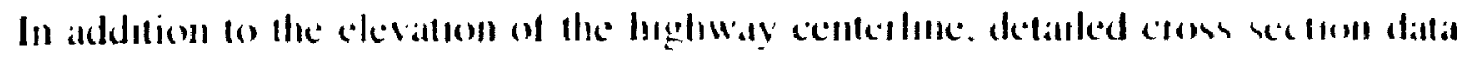

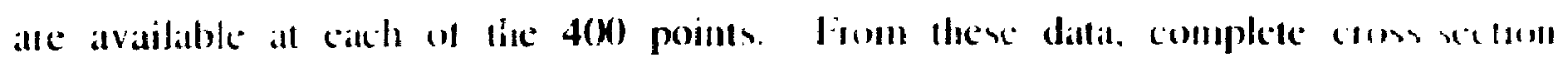

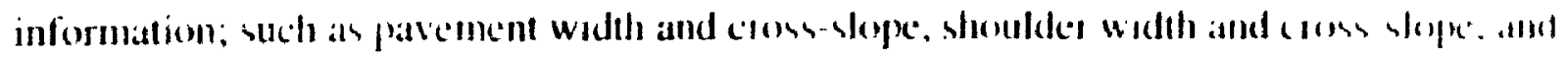

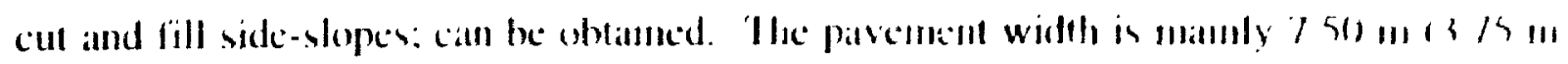

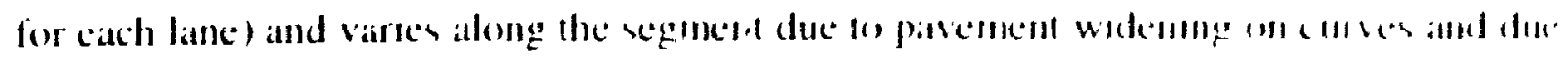

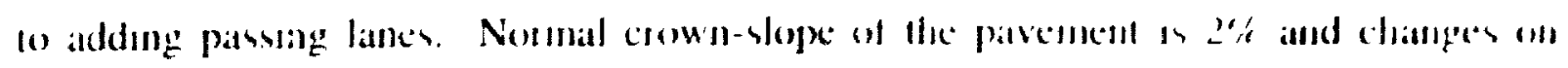




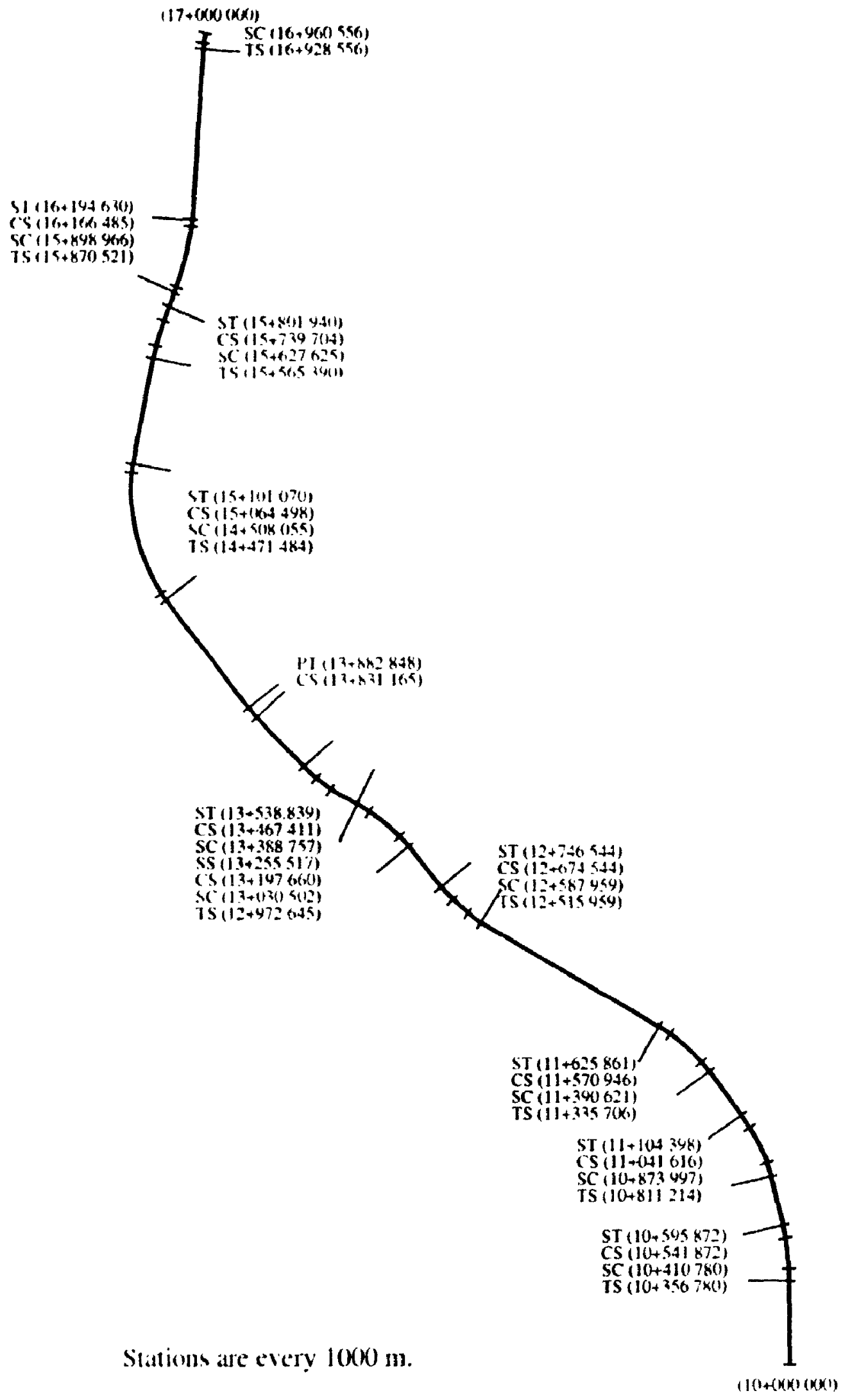

FIGURE 6.2: Horizontal Aligiment of Highway Segment. 


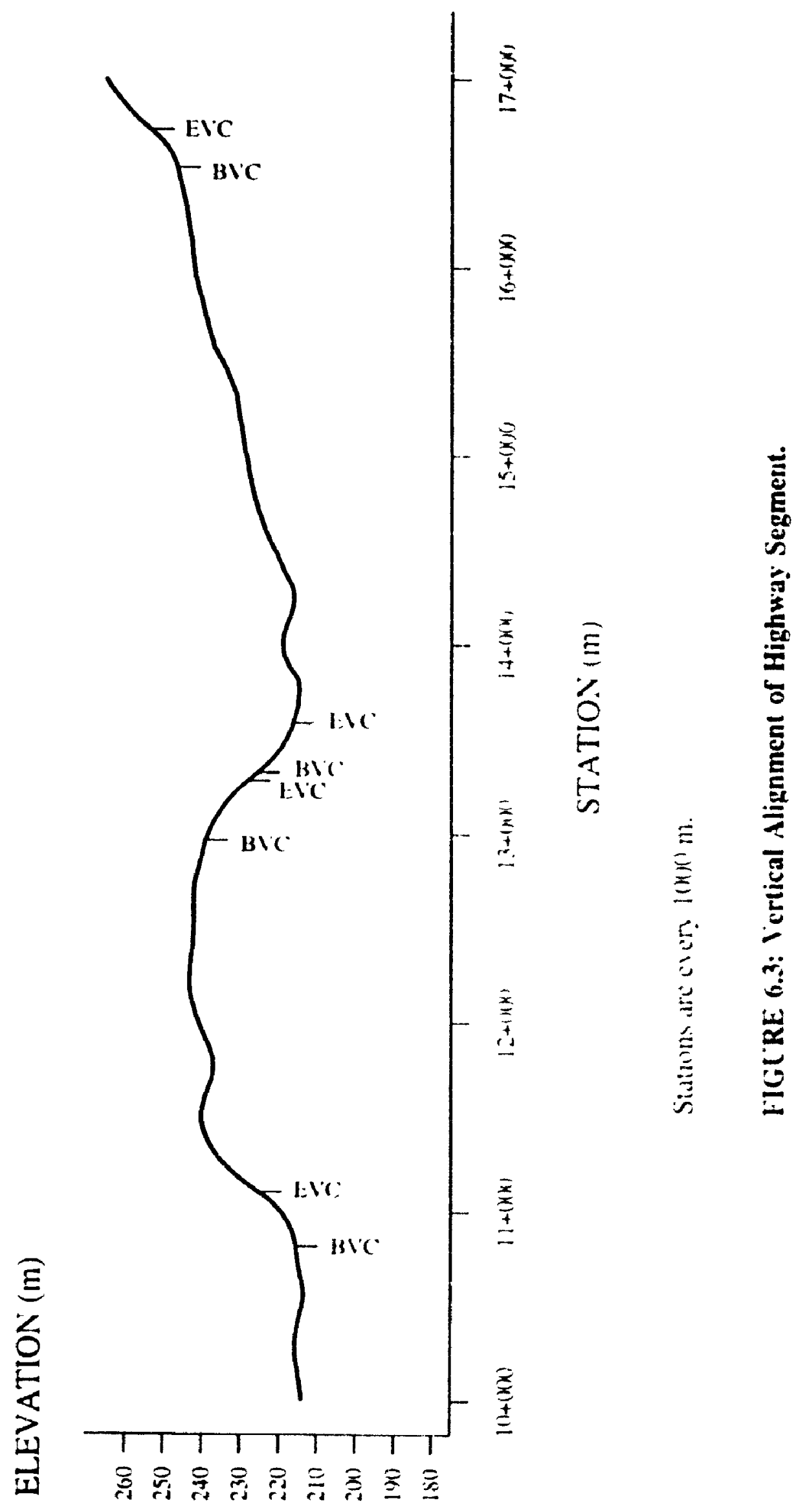


curves to the superelevation rate (up to $5.90 \%$ ). Shoulder width is mainly $2.5 \mathrm{~m}$ but varies up $101.5 \mathrm{~m}$. Shoulder cross-slope is mainly $6 \%$ and varies on curses depending on the superelevation rate. Finally, side-slopes vary widely and include fill in both sides, cut in both sides, fill in one side and cut in the other, and vertical cut in rock areas. However, in cut sections, a down-slope (with or without a ditch) is introduced before the up-sope. Figure 6.4 shows examples for four different cross-sections. In defining the cross section data to the computer sofiuate, a totul of 189 points (ciut of the original 400 poimts) were used. The cross-section data for any other point is estimated by linear interpolation.

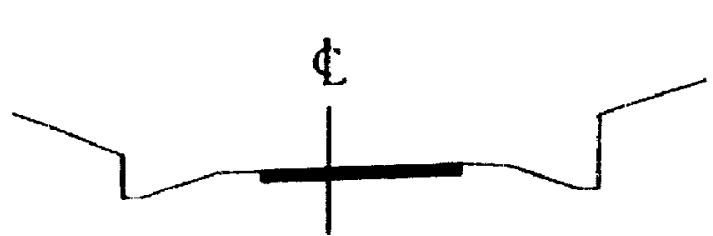

(a) Station $(11+38())$.

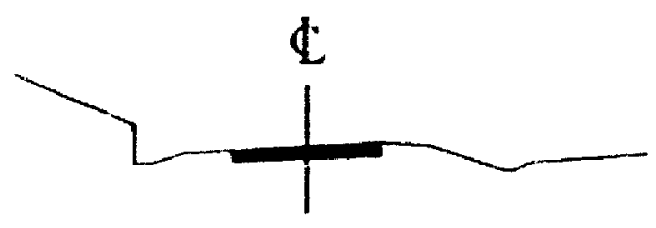

(c) Station $(13+(150)$.

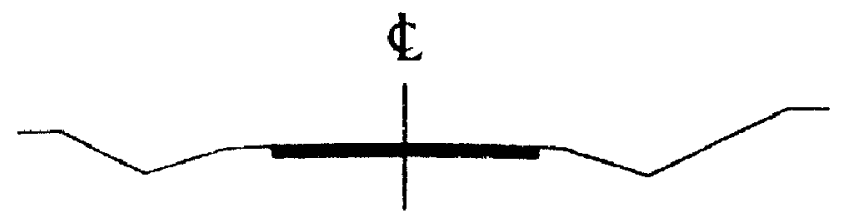

(b) Station $(13+936)$.

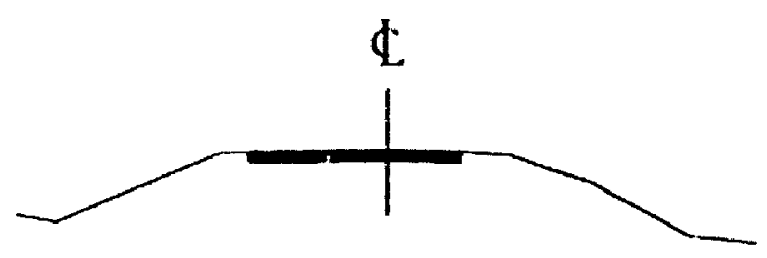

(d) Station $(15+839)$.

FI(i) 'RE 6.4: Examples for Different Cross-Sections of Highway Segment. 


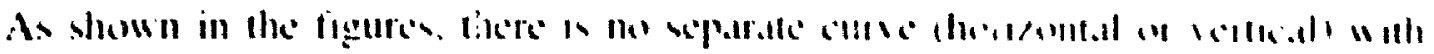

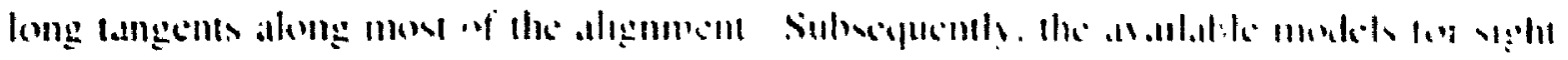

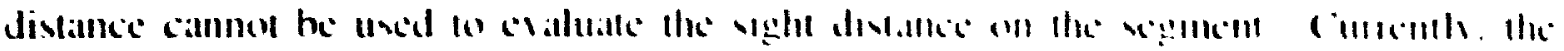

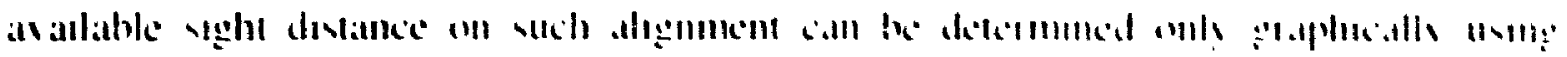

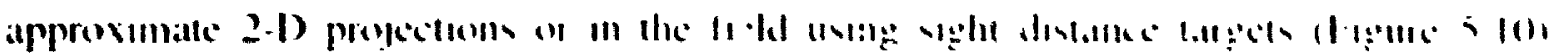

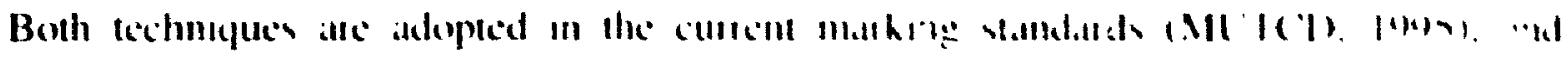

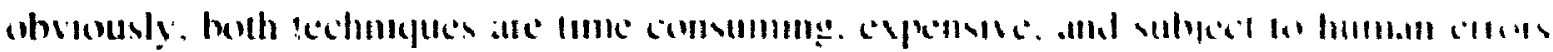

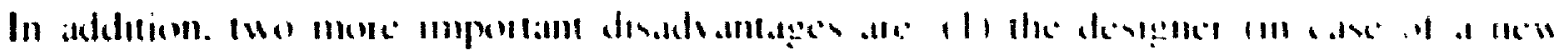

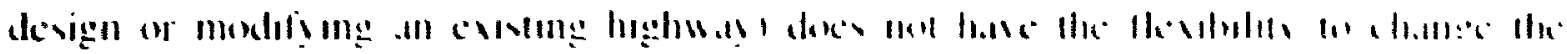

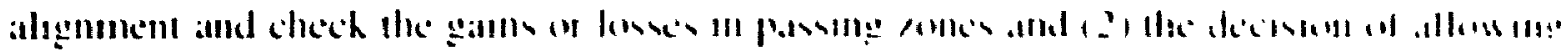

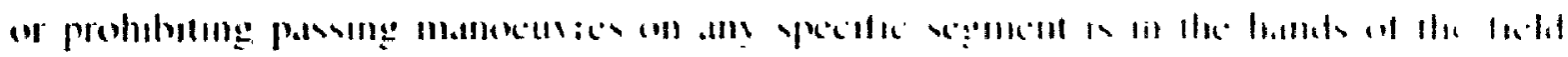

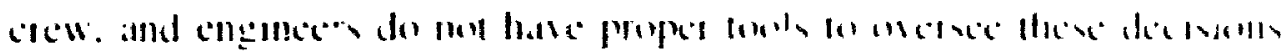

\subsection{Application Chjectives and Procedure}

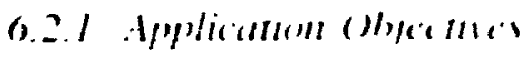

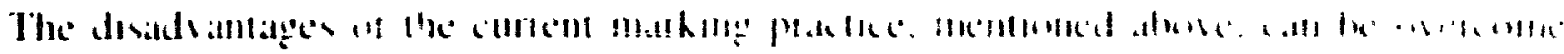

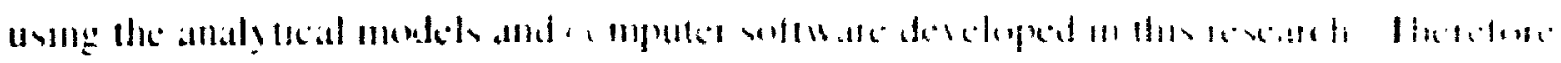

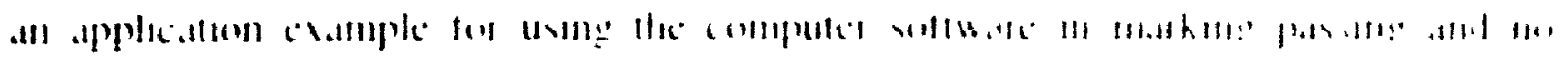

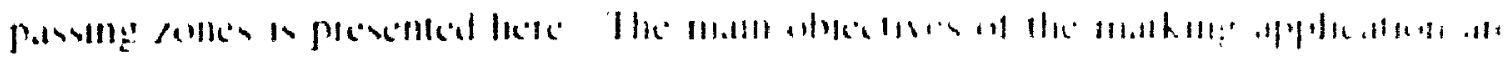

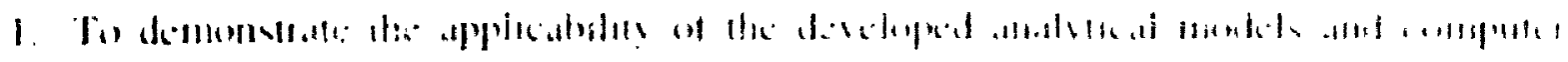

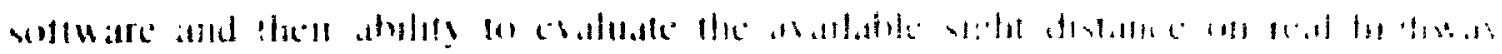
x'gullonts 

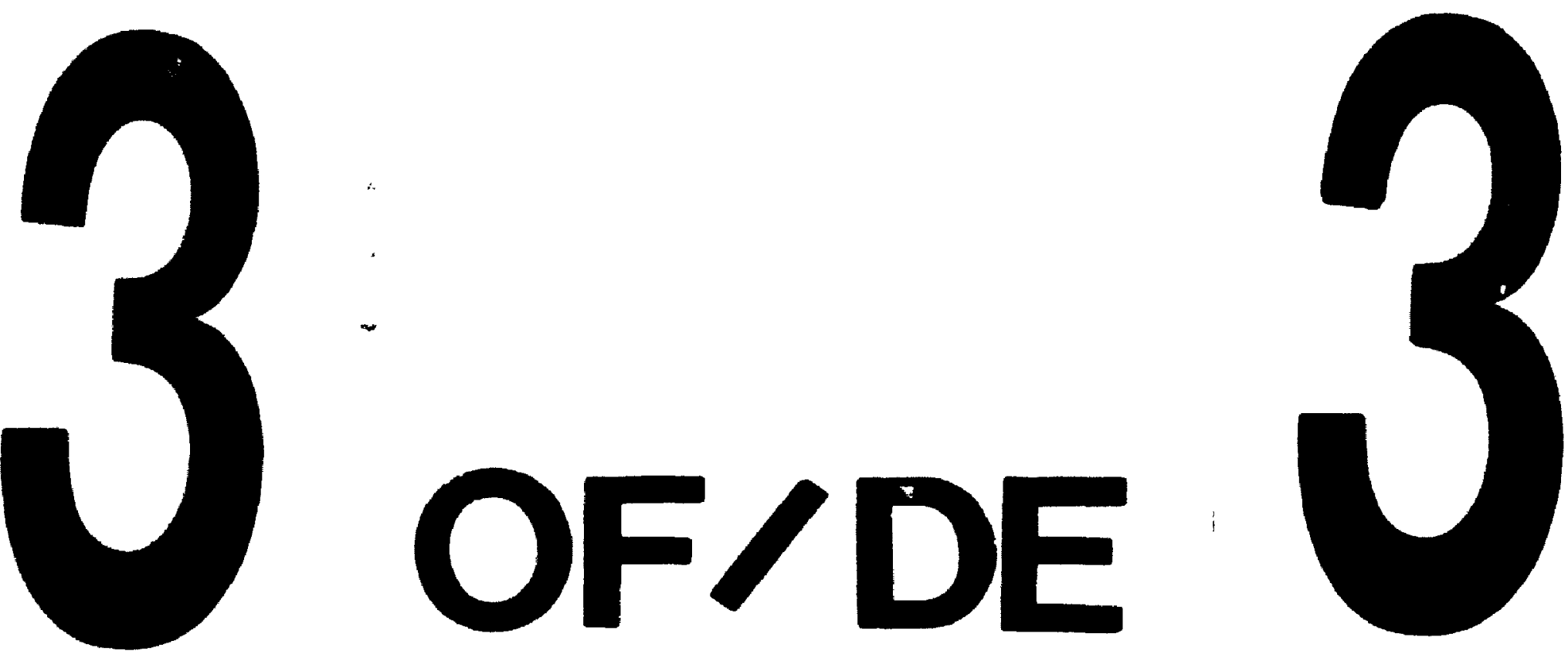

PM-1 3', "x " PHOTOCRAPHIC MICAOCOPY TARGET NES 1010a ANSH/ISO \#2 EOUIYALENT

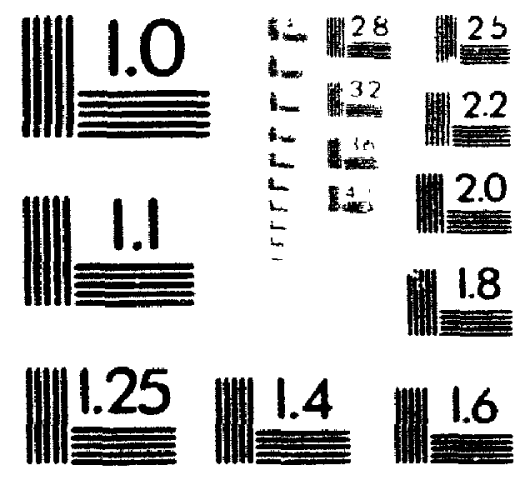

PAECISIONSM RESOLUTION TARGETS 
2. To pinpoint the important points that should be taken eare of "lewt using the developed software. specifically, alignment preparatum. element sise, amd eomputer rin-time.

3. To compare between the 2-D and 3-1) avdilable sight distances and 10 yuatuty the range of error associated with ignoring the 3-D nature of highway alignments.

4. To compare the marking obtained by the developed sofiwate wilu the curremt matrkin! in the field.

5. To compare the marking according to the MlleCD (194), tandards with that according to the criteria developed in this research (Chapler 3).

\subsubsection{Compater Programs and User-.Specified Parameters}

Because of the changes in the pavement width and latcral obstruction due to cull slopes (horizontal alignnent) and because of the spline grades (vertical alignment), both $M A R K H$ and MARKV could not be used in this application. Thus, MARKS wat used In thus application to evaluate the available sight distance, $S_{a}$. on 2-I) separate horizontal and vertical alignments. On the other hand, MARKC was used to evaluate $S_{\text {in }}$ (1) the 3 I) combined alignment. As explained previously, in both programs. the user specifics certain parameters which control the minimum sight distance to be checked, $S_{m}$, accuracy of the calculated sight distance. ACC. and the range of stations on which the sight distance should be evaluated (first and last station and step between each two successive stations). 
Although the current marking tandards specifies a $300-\mathrm{m}$ minimum PSD corresponding to a speed limit of $90 \mathrm{kph}\left(\right.$ ML TCD. 1995), $S_{m}$ was specified as $400 \mathrm{~m}$ which corresponds w the minimum PSD developed on the research (Figure 3.9). Therefore, the range of stations on which $S_{a}$ could be calculated was $10+000$ to $16+600$. for the right lane, and $10+4(4)$ to $17+000$, for the left lane. The step between each two successive stations, STEF, was set as $10 \mathrm{~m}$. Thus, $S_{a}$ was determined on a total of 661 tations on each lane. Finally. ACC was set as $1.0 \mathrm{~m}$. However. to speed up the computer runs, $S_{a 1}$ was checked using $100-\mathrm{m}$ jumps. If the sight distance was obstructed. the finer $1.0-\mathrm{m}$ jump was used to determine $S_{a 1}$ to the required accuracy.

\subsubsection{Experimemal Design}

Referring to the parameters set above. a single computer run involved the calculation of $S_{a 1}$ to the nearest $1.0 \mathrm{~m}$ on a total of 1.322 stations. In order to achieve the objectives listed earlier, a four-phase study was carried out as follows.

Phase (1): Efrect of Element Size. Reducing the element size increases both the modelling accuracy and the computer run-time. While the former is desirable, the latter is not. Therefore, different element sizes were used to study the trade-offs between the modelling accuracy and computer run-time in 2-D and 3-D alignments. Three element sizes: namely. 10. 25. and $50 \mathrm{~m}$ : were used to evaluate $S_{a \mathrm{~m}}$ in 2-D separate horizontal alignment. 2-D separate vertical alignment. and 3-D combined alignment (total of 9 tuns). It should be noted. however. that these element sizes are the maximum size that can be 
used for any small element (om horizontal curses or vertical splute grates). The real element size is variable and depends on the lenght of eith segment hetweon mo successive points used to define the horizontal algenment. the vertabl alignment. an the cross-section. Also, this element size dokes mot control the long elements used an stratight segments or parabolic vertical curves. The heights of the driver eye and the olved. ht and $h_{2}$ were taken as 1.05 and $1.30 \mathrm{~m}$. respectively (T.AC, $198(6)$.

Phase (2): 2-D Versus 3-D Sight Distances. The same rums in the tirst plate can lec used to compare the 2-D and 3-1) sight distances. Thus. the effect af ignowilg llie 3 1) nature of highway alignment regrading the sight distance can be quantified.

Phase (3): Comparison with Existing Marking in the Field. According to the curremt marking standards (MUTCD, 1995). a no-passing zone shumld begin on verlacal alignments when a $1.05-\mathrm{m}$ object is not visible 10 a driver eye all $10.5-\mathrm{m}$ height. However, the same no-passing zone should end when a $(1.30$-m objecl is visible 10 a driver eye at $1.05-\mathrm{m}$ height. On horizontal alignments, a comstant ohject height at 0.30 $\mathrm{m}$ is used. Therefore, two computer runs with 1.05 and $(1.30)-11$ object heights wetc carried out on the 2-D separate vertical alignment. Another rull with a 1 . 30-111 object height was carried out on the $2-D$ separate horizontal alignment (tostal of 3 rums). In ihus last case, a continuous lateral obstruction after a $3-\mathrm{m}$ shoulder is added on right-lurn curves to simulate the MUTCD (1995) standards used by MTO. The element size in each of these runs was taken as the optimum size determined in the first phase. 
Phase (4): Marking Esing Profiles of A vailable and Required PSD. In this phase. practacal marking considerations developed for marking passing and no-pas:,ing zones (chapter 3 , are used w th the profile of $S_{a t}$ to establish the optimum marking. The driver eye and object heights were taken as 1.05 and 1.39$) \mathrm{m}$. respectively. It should be noted that the shject height of $1.05 \mathrm{~m}$ is set in the MIFTCD standards to mark the two lanes at the same time and reduce the amount of work. which is not a matter of concern when using the computer software. Since these runs were already included in the first phase. no alditional runs are require in this phase.

In summary, a total of 12 runs were conducted in this application. The main parameters in these runs are summarized in Table 6.1 .

TABILE 6.1: Summal of Experimental Design.

\begin{tabular}{lccc} 
Parameter & \multicolumn{2}{c}{ 2-D Separate Alignment } & $\begin{array}{c}\text { 3-D Combined } \\
\text { Alignment }\end{array}$ \\
\cline { 2 - 4 } & Horizontal & Vertical & MARKC \\
\hline Software & $M A R K S$ & MARKS & MA. \\
Element size $(\mathrm{mi}$ & 10.25 .50 & 10.25 .50 & $10.25,50$ \\
Height of driver eye. $h_{1}(\mathrm{~m})$ & 1.05 & 1.05 & 1.05 \\
Height of object, $h_{2}(\mathrm{~m})^{a}$ & 1.30 .0 .30 & $1.30,1.05,0.30$ & 1.30 \\
Number of runs & 4 & 5 & 3 \\
\hline
\end{tabular}

“ Only $h_{z}$ of $1.30 \mathrm{~m}$ is used with the three element sizes. Other values of $h_{2}$ are used with a specific element size 


\subsection{Results and Analysis}

\subsubsection{Phase 1: Effect of Ele'ment Size}

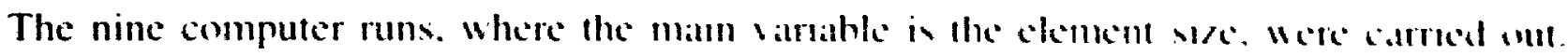
Figure 6.5 shows the element net created by M:1RKS w mokel the l.tter.t obstuctums due to th: cut-slopes in the 2-D horkental alignment using a $25-\mathrm{m}$ maximum telement s/e:, white the element net to model the highway surtite un the 2-1) vertical athgument is shown in Figure 6.3. An example for the element net created by MARKR to makel the highw:ay surface and side-slopes in the 3-D combuned alignment usmg a 25-II maximum, element size is shown in Figure 6.6.

The computer run-time for each run is shown in Tathle 62 . The lable shows also the effect of the element ste on the calculated avalable sight distance. $S_{11}$. The smalle's element size of $10 \mathrm{~m}$ is used as a reference and the effect of any ofher specilic element size. Eff. is defined as:

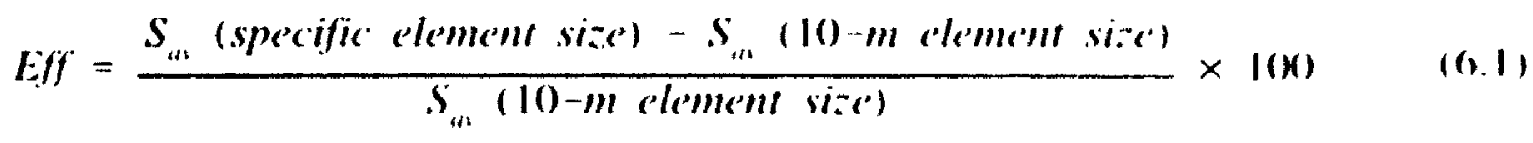

where Eff can be positive or negative.

As shown in the table, expectedly. reducing the element sise mereatses the computer run-time. However, the rate of increase in run-tume varies in each of the three cases show. in the table (2-D horizontal. 2-D vertical, and 3-1) combuned). Hart. smce: most of the vertical alignment is spline grade, and is modelled by shorf clements, reducing the element size will increase the number of elements and run-fime wilh almost the same ratio. However, because the parabolic curves and tangents are modelled with 


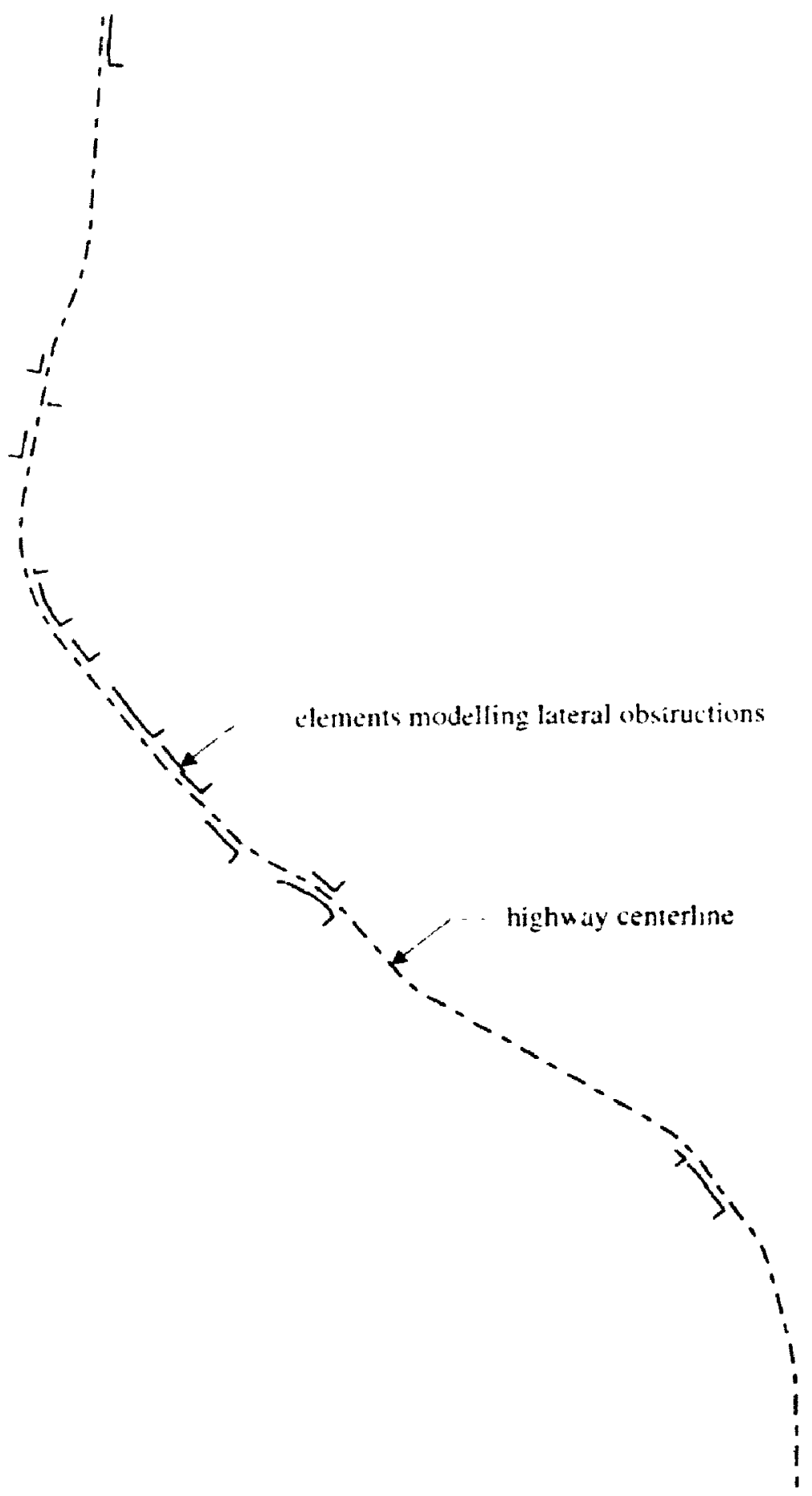

FIGURE 6.5: Element Net for 2-D Horizontal Alignment. 


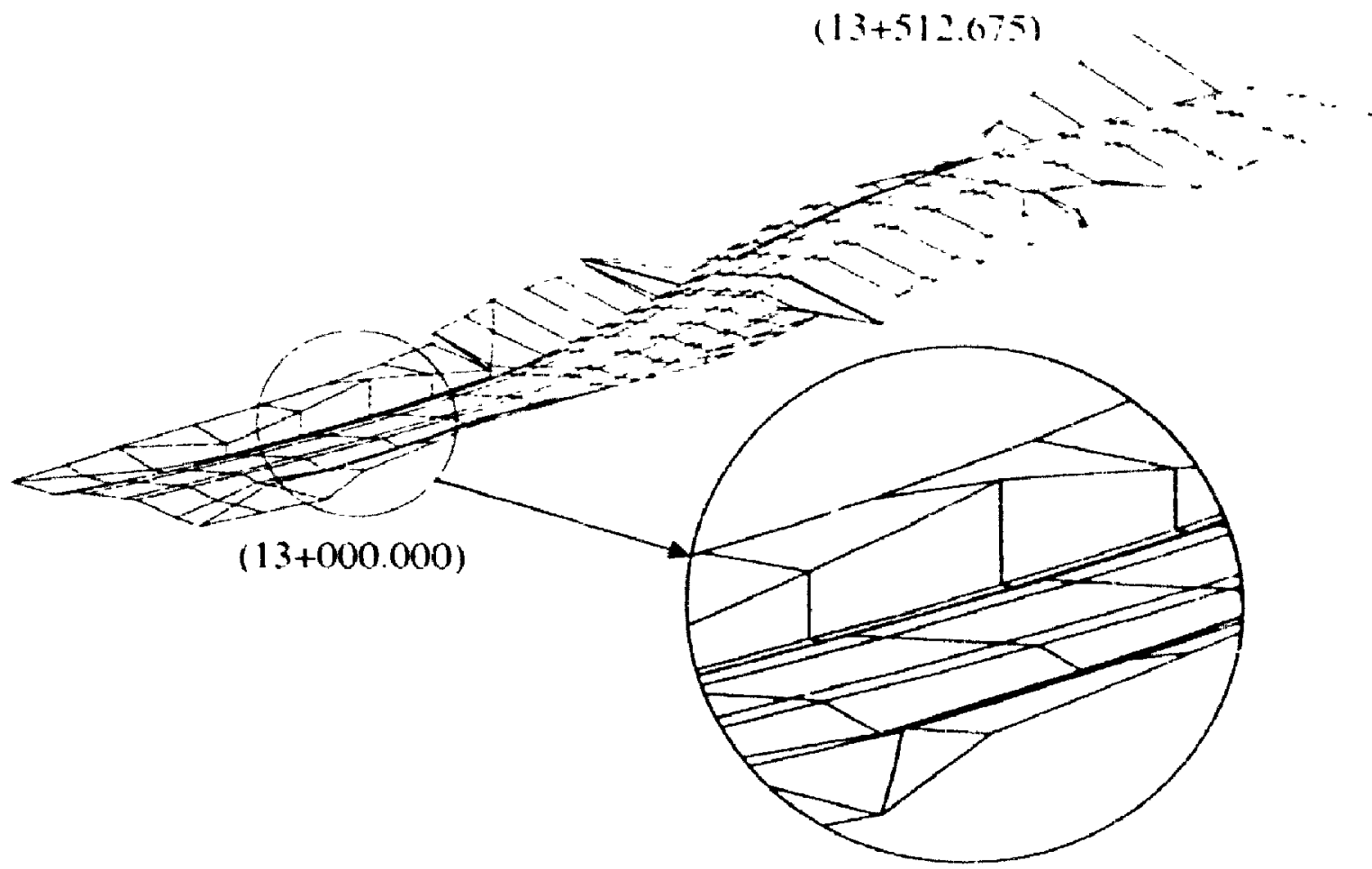

FIGURE 6.6: Example of Element Net for 3-1) (ombired Alignment.

\section{TABLE 6.2: Effect of Element Size.}

\begin{tabular}{lccc}
\hline \multirow{2}{*}{ Element Size } & \multicolumn{2}{c}{ 2-D Separate Alignment } & 3-1) ('omhined \\
\cline { 2 - 4 } & Horizontal & Vertical & Alignment \\
\hline (a) Computer Run-Time (minutes) & & \\
$10 \mathrm{~m}$ & 3.77 & 7.38 & 3.1202 \\
$25 \mathrm{~m}$ & 2.77 & 3.28 & 16.4 .53 \\
$50 \mathrm{~m}$ & 2.55 & 1.88 & 117.78 \\
\hline
\end{tabular}

(b) Eff (Equation 6.1).

\begin{tabular}{llll}
$25 \mathrm{~m}$ & -2.20 to $+0.90 \%$ & -0.48 to $+1.02 \%$ & $-0.46 \mathrm{to}+0.51 \%$ \\
$50 \mathrm{~m}$ & -2.19 to $+50.57 \%$ & -0.98 to $+3.11 \%$ & $-1.13 \mathrm{t} \%+086 \%$ \\
\hline
\end{tabular}

a Using a 486-PC with $66 \mathrm{MHZ}$ speed 
anc elemem cach, regardless of the maxtmum element vize. the rattes of increatse in number of clements and ran-tume aic slightly less than the rate of reduction in element 41/. As for the horizontal alignment. a large portuon of the alignment consists of tangents where the clement sise does not depend on the maximum size. In addition, each point used to define the horrontal alignment or the cross-section must fall at the end of an element and the heginning of a new element. The large number of points used in delimng the cros- section data (189 points) would produce an average element length of $3 \% .2 \mathrm{~m}$ regardless of the maximum size. Therefore. the rates of increase in number of elements and run-time are consderably less than the rate of reduction in elenent size. The samk argument is also valid for the 3-D combined alignment.

As shown also in the table. the low values of Iff mdicates that increasing the clement si/e from 10 to $25 \mathrm{~m}$ would result in slight reduction in the modelling accuracy of 2-1) and 3-D alignments. The same argument is also valid for 2-D vertical alignment and 3-1) combined alignment when the element size is increased from $101050 \mathrm{~m}$. On the wher hand. the $5(0)-m$ maximum element size produced a considerably high value of Eff $(50.57 \%$ ) in case of 2-D horizontal alignment. This high value of Eff occurred at one statton only $13+05($ on the right lane $)$ and the next highest value of Eff was $6.17 \%$. The reason for this high value of Eff can be referred to the modelling of the lateral obstruction hetween station $13+100$ to $13+! 50$. Cross section data are defined at both stations and are interpolated for any intermediate station. The exact lateral clearance from the highway centerline to the left lateral obstruction produced by the cut-slope is constant (4.41 $\mathrm{m}$ ) from ; on $1.3+100$ to $13+140.8$. and then increasing gradually to $12.212 \mathrm{~m}$ at 
station $13+150$. As shown in Figure 6.7, this profile of lateral clearance 11 as modellad almost exactly using 5 elements of $10 \mathrm{~m}$ alement sike each. However, fior a $50 \mathrm{~m}$ element size, only one slement was generated to mokel the obstruction. Nhhough the element had the exact lateral clearance at $13+100.13+125$, and $13+150$. usmp a ungle curve to fit these three points slightly underestimated tive lateral clearance form wation $13+100$ to $13+125$ and overestimated it from station $13+125$ to $13+150$. This falled overestimation of the lateral clearance caused the sight distance on station $13+(1) i d$ (w) be overestimated from $206 \mathrm{~m}$ to unobstructed $(f(H) \mathrm{m})$.

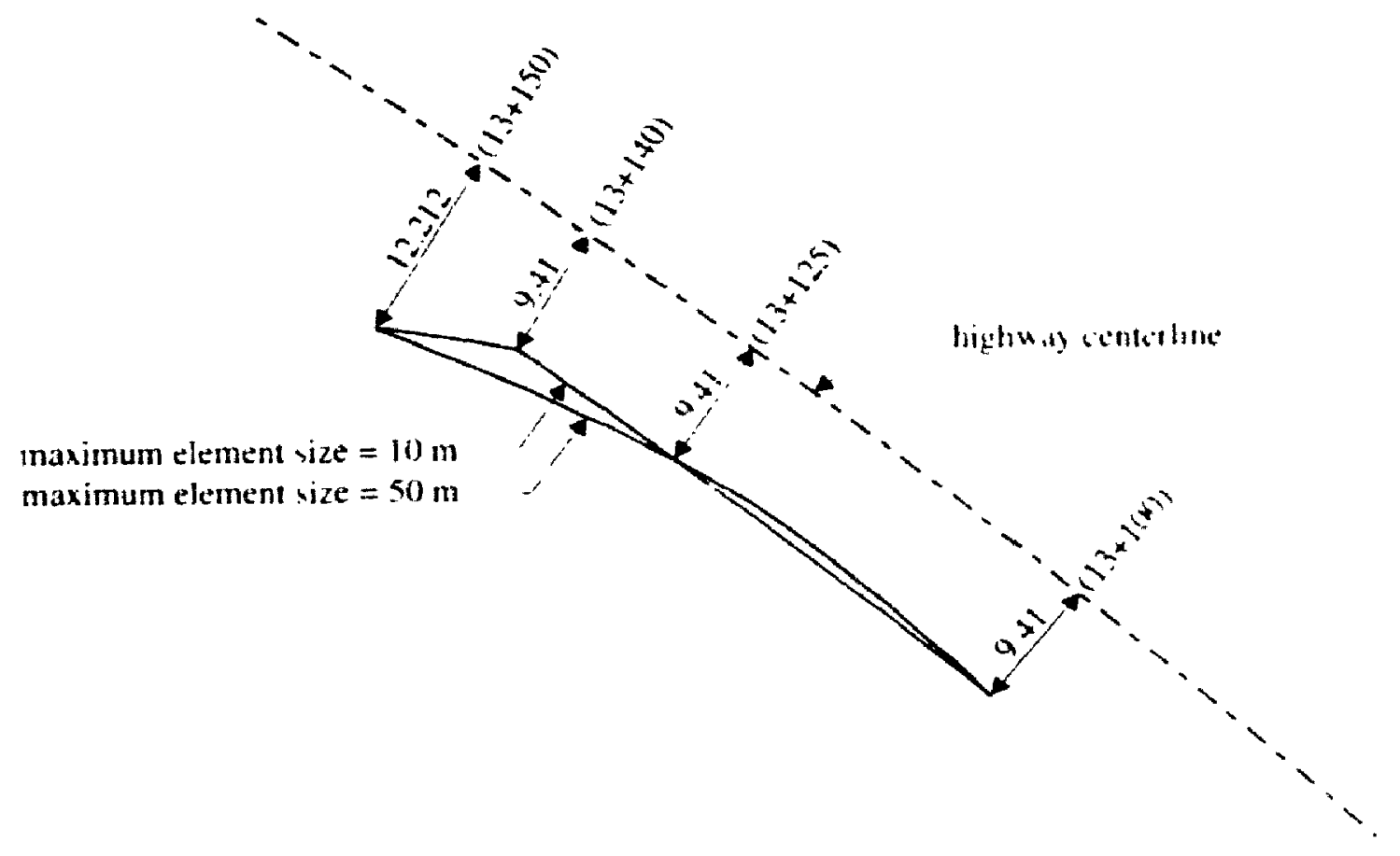

FIGURE 6.7: Lateral Obstruction in 2-D Horizontal Alignment from Station $(13+100)$ to Station $(13+150)$. 
It should be noted. however. that this problem was associated with the 2-D horizontal alignment with variable side-slope data. For the 3-D combined alignment. using surfaces to model the side-slopes did not cause the same accuracy problem. Moreover, the values of E:ff in the case of 3-D combined alignment were considerably less than those in both $Z-D$ horizontal and vertical alignments. This shows that the 3-D modelling produces a relatively better accuracy which is less dependent on the element vire.

In summary. a caution :hould be practiced in selecting the element size in 2-D horizontal alignments. Noting that the run-time in this case is significantly short. small element size is recommended if the side-slope data vary along short distances. On the other hand. the element sire does not have a signticant effect on the modelling accuracy of 2-D vertical alignments or 3-D combined alignments. For this specific segnkent, a 25 In elenent size represents the best compromise between the run-time and the accuracy.

\subsubsection{Phasc 2: 2-l) V'ersus i-D Sight Dis.ances}

In this phase. the results of sight distance obtained in the first phase for a $25-\mathrm{m}$ clement length are used to compare the 2-D and 3-D alignments. At any station. the 2-D sight distance or 2-D PSD refiers to the minimum of the two values of passing sight distance (PSI) determined from 2-D horzontal and vertical alignments. The corresponding sight distance defermmed from the 3-D combined alignment is referred to as the 3-D sight distance or 3-1) PSD. Profiles of both 2-D and 3-D PSD are shown in Figure 6.8, and 


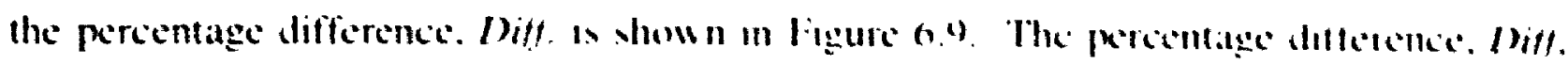

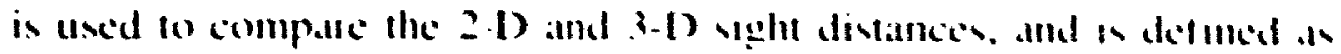

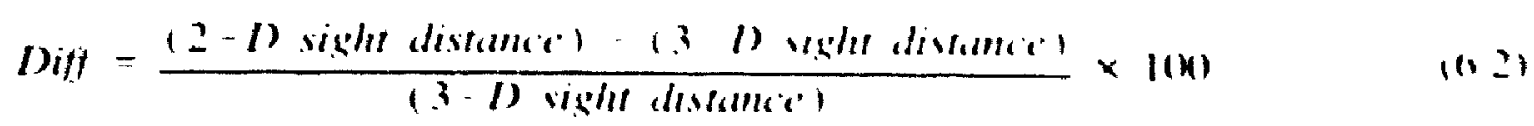

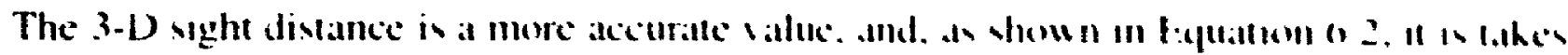

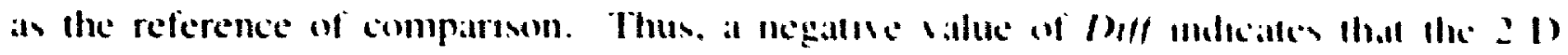

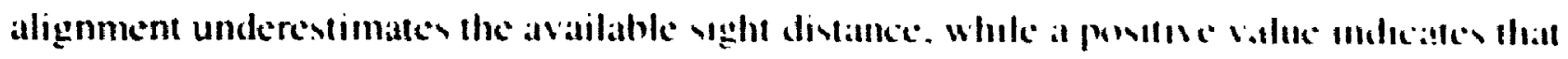
the 2-D alignment orerestimates the at alable vght devtance.

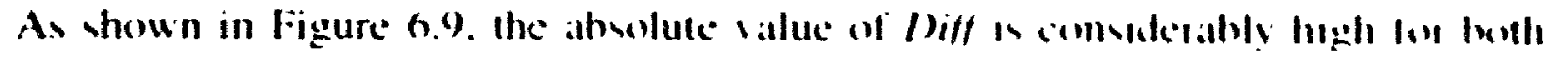

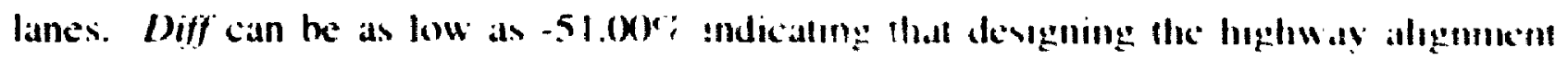

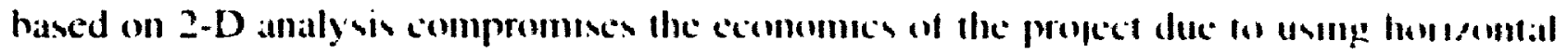

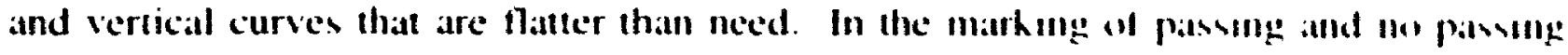

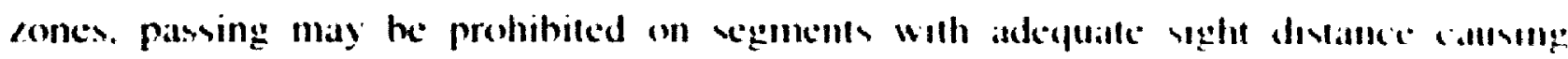

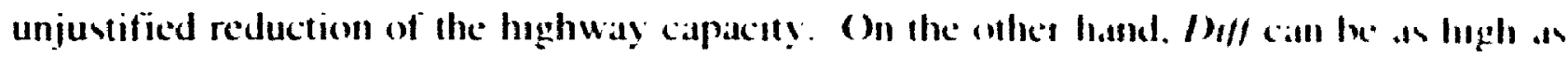
$11.51 \%$ indicating that designing the heghway alignment bated on 21 allalysu compromeses the traffic safety due to using horecontal and werteal curver that ale vhatpel than needed. In the marking of passing and no-passong /ances, passmy maty be allowed on segments with inadequate sight distance generattug unsate passmg mankeuses A close investigation of $l$ Sitf with the hortental and vertical alignment and croms section data showed that the positive values of lliff were encountered at comburmed horizontal and crest vertical curves in cut sectums. In such cabes, the crest verllacal curves cause both the driver eye and the object to sink relative to the latcral ohstructinl. Ilius. 


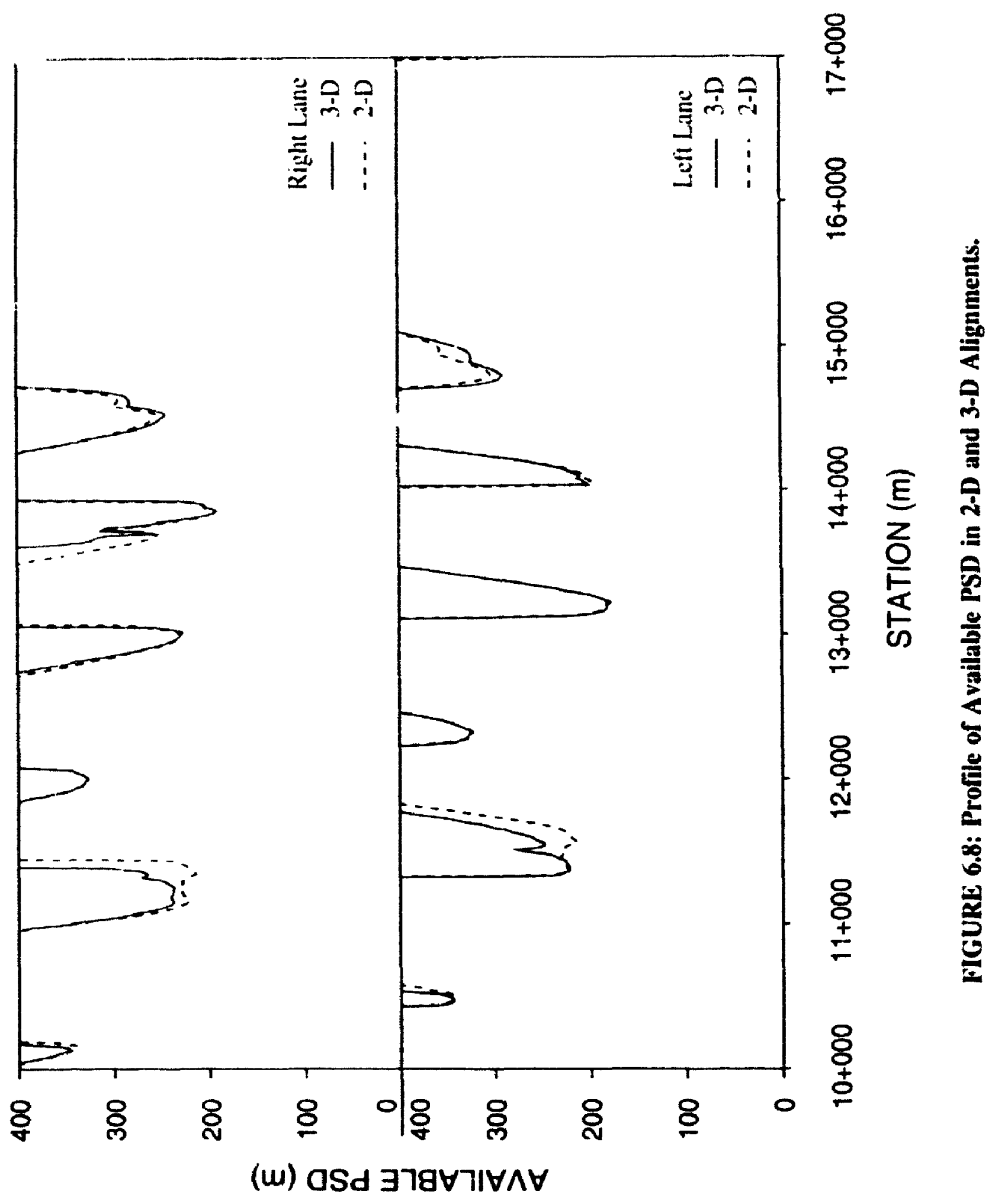




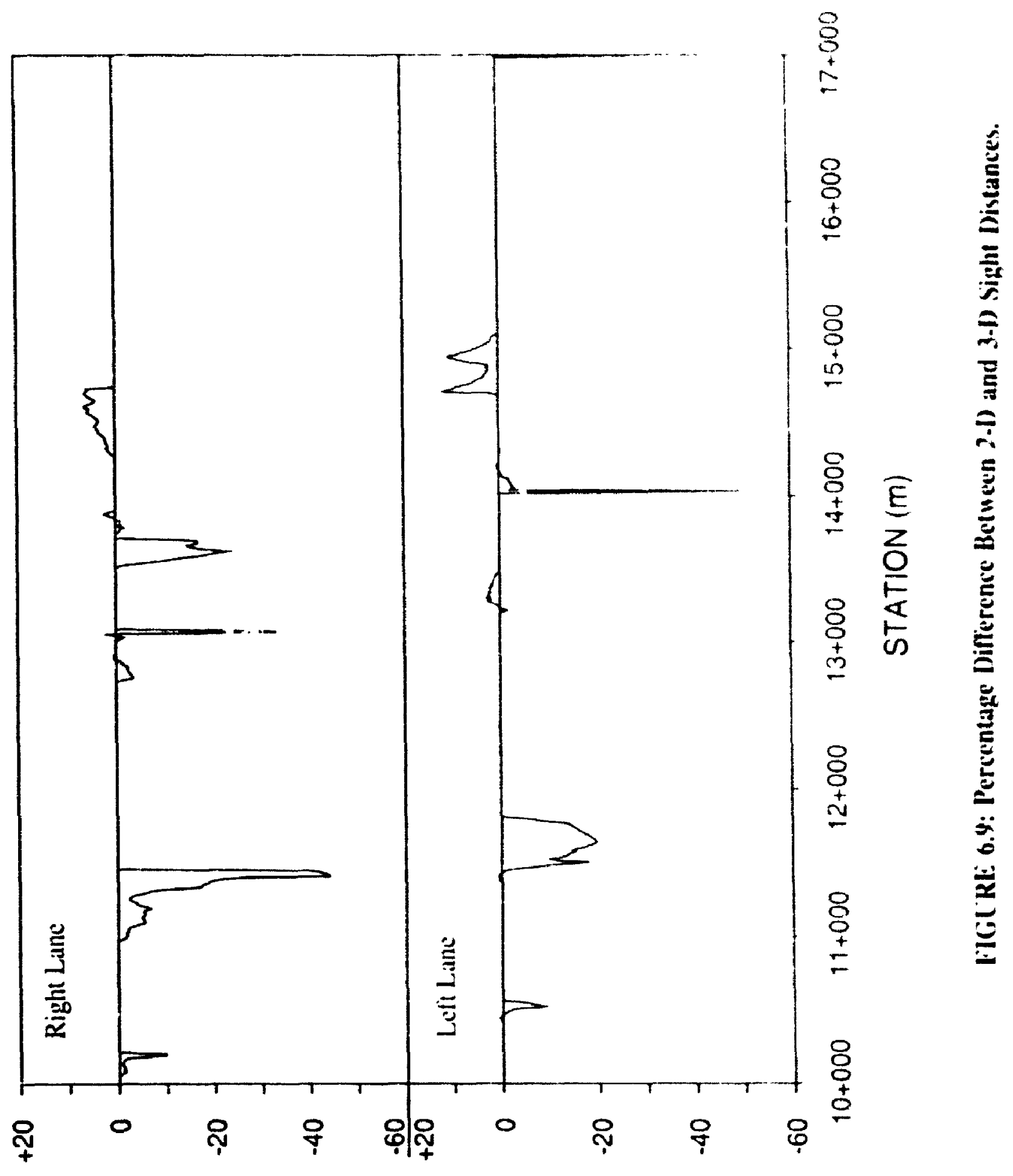

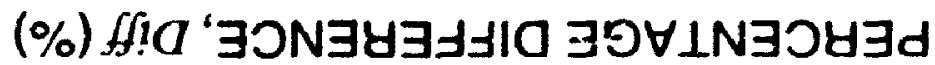


the lateral clearance at which the sight line becomes tangent to the lateral obstruction is less than the laterit clearance calculated at the average height between the driver eye and the object in 2-D horizontal alignment. On the other hand, the negative values of Diff were encountered at two situations, combined horizontal and sag vertical curves in cut sections and combined horizontal and crest vertical curves in fill sections. The first case is the opposite of the case with positive Diff, whele the sag curve causes the effectivg lateral clearance to be greater than that calculated at the average height between the driver eye and the object in 2-D horizontal alignment. In the second case, the superelevation on the horizontal curve lowers the highway surface relative to the sight line. Therefore, the crest vertical curve becomes less critical than it looks to be in 2-D vertical alignment. These three cases are discussed in more detail in Chapter 7.

\section{3.. Phase 3: Comparison with Existing Marking in the Field}

As explained earlier, the profile of available PSD obtained by MARKS and MARKC can be used to determine the marking of passing and no-passing zones on two-lane highways. Figure 6.10 shows the profile of available PSD in 2-D a:ignments according to MUTCD (1995) standards used by MTO. According to these standards, the minimum required PSD, corresponditg to a 90-kph speed limit, is $300 \mathrm{~m}$. The available PSD at any specific station is the lower of PSD on horizontal and vertical alignments. In calculating the PSD on the horizontal alignment, the object height, $h_{2}$, is set as $0.30 \mathrm{~m}$ and a vertical continuous ohstruction after a $3-\mathrm{m}$ shoulder is added on right-turn curves. For the vertical alignment. $h_{z}$ is initially set as $1.05 \mathrm{~m}$. If a no-passing zone is detected $\left(S_{w}<300 \mathrm{~m}\right)$, 


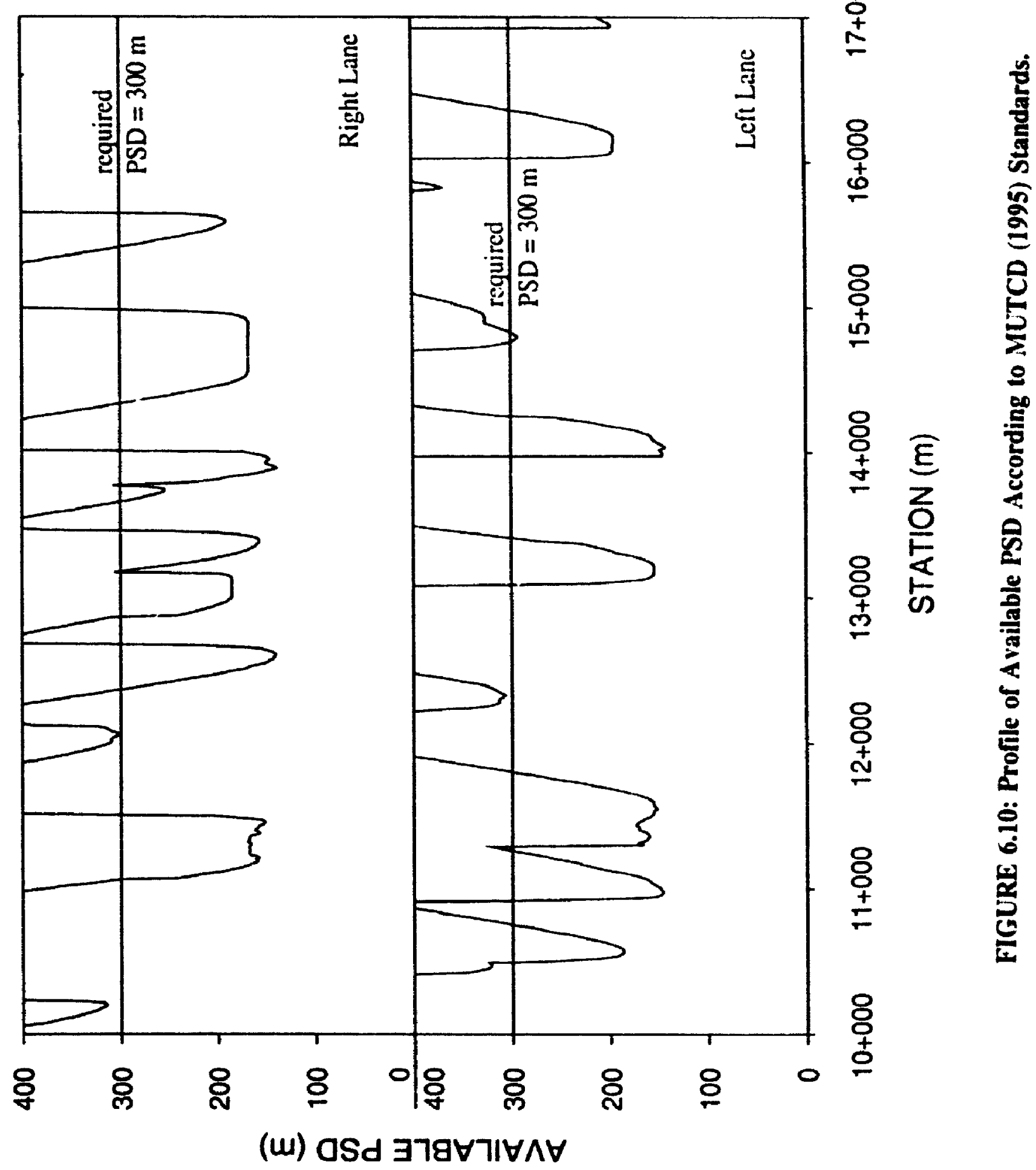


$h_{2}$ is set as $0.30 \mathrm{~m}$ until the no-passing zone ends where $h_{2}$ is re-set as $1.05 \mathrm{~m}$. As shown in the figure, a no-passing zone should be marked wherever the available PSD drops below the 300-m minimum required PSD.

Additional considerations in marking passing and no-passing zones are (MUTCD, 1995):

1. The minimum length of passing zones, $l_{z}$, is $280 \mathrm{~m}$. If a passing zone has a length less than $280 \mathrm{~m}$, passing should be prohibited and a no-passing zone is warranted. This warrant was not considered at the segment's both ends.

2. The minimum length of no-passing zones is $150 \mathrm{~m}$. If a no-passing zone has a length less than $150 \mathrm{~m}$, an additional length should be added to the beginning of the nopassing zone This warrant was not considered at the segment's both ends.

3. When a passing or a truck climbing lane is added in a specific direction, a no-passing zone should be marked in the same direction. The no-passing zone begins $100 \mathrm{~m}$ before the beginning of the widering taper and ends at the end of the narrowing taper (if the sight distance is unobstructed). However, the opposite direction can be marked as passing or no-passing depending on the available PSD. Therefore, a no-passing zone is warranted from station $10+640$ to $12+550$ on the right lane and from station $17+000$ to $16+420$ on the left lane.

4. A no-passing zone should be marked on bridges which are not $1.25 \mathrm{~m}$ or more wider than the approach pavement. The no-passing zone begins $120 \mathrm{~m}$ before the bridge structure and ends at its end (if the sight distance is unobstructed). Therefore, a no- 
passing zone is warranted from station $13+6.30$ to $13+776$ on the right lanc and from station $13+896$ to $13+750$ on the left lane.

A summary for the marking of passing and no-passing zones obtained using the profile of available PSD is shown in Table 6.3. If a no-passing zone is warratited at a specific segment, the warrant is given in the table. The actual marking in the field was recovered by the MTO Traffic Section, and is shown in Table 6.4. Comparing the marking in Tables 6.3 and 6.4 shows that the marking based on the profile of PSD matched all the passing zones in the field. However, a considerable length that is safe for passing according to MUTCD (1995) standards is marked as no-passing in the ficta. Although, ignoring the single obstructions in the computer runs may contribute to this difference, the consideration of a continuous obstruction after a $3-m$ shoulder on right curves reduces this possibility significantly. On the other hand, there are some no-passing zones where the warrants are not obvious and could not be lateral obstructions. For example, on the right lane in the field, a no-passing zone begins at station $16+532$ while the segment remains straight in the horizontal alignment until station $16+928.556$. In the vertical alignment, the segment consists of a sag curve followed by a langent and a smooth crest curve that does not obstruct the sight distance until station $16+680$. In addition, no intersections are encountered at this specific section. Thus, this los; of almost $150-\mathrm{m}$ passing zone is not justified. 
TA BI.F 6.3: Marking Using Profile of Available PSD and MUTCD (1995) Standards.

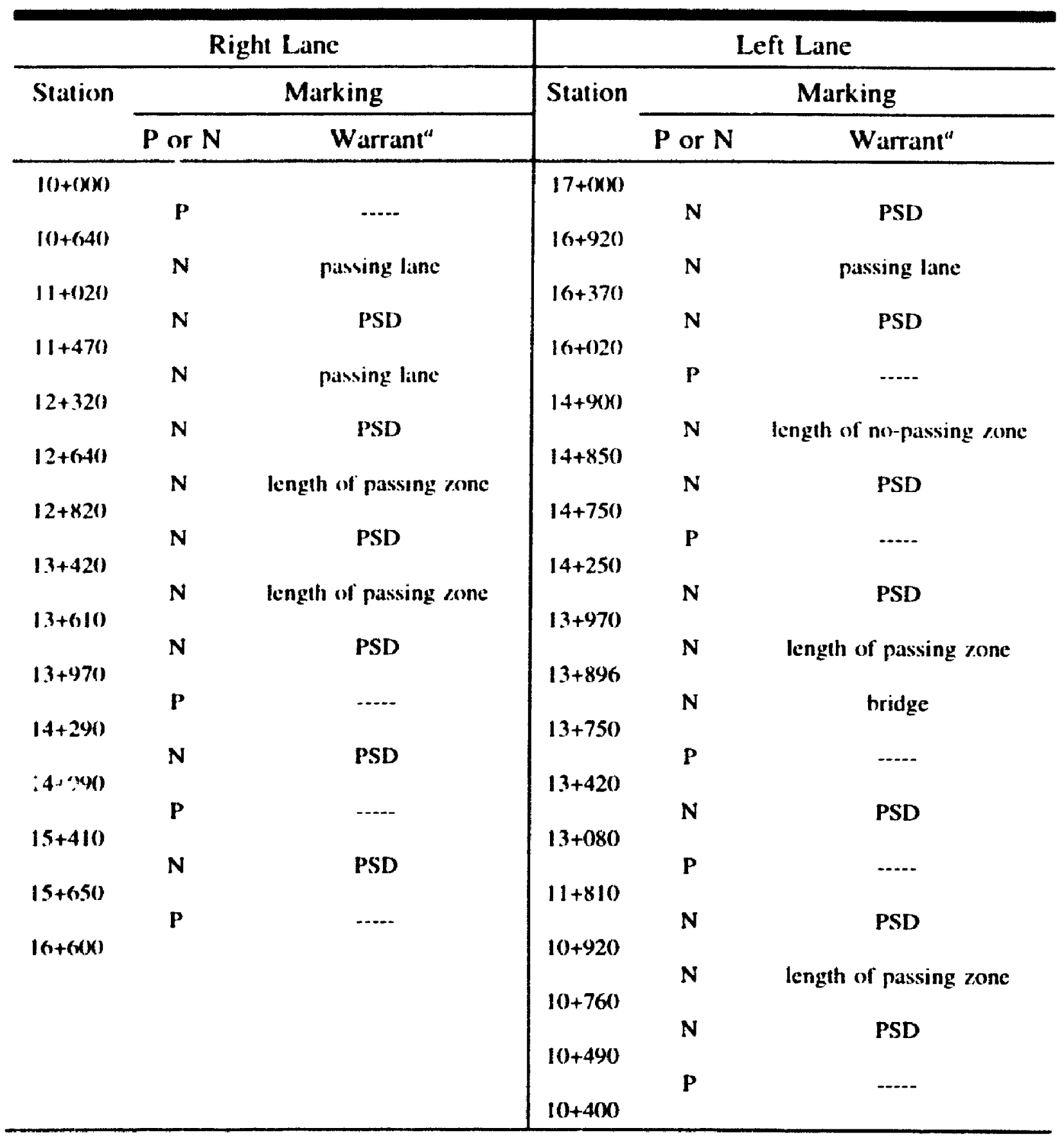

$\mathbf{P}=$ passing zone. $\mathbf{N}=$ no-passing zone

Right and left lanes are relative to the direction of increasing stations

"Warrant for no-passing zones 
TABLE 6.4: Segment Marking in the Field.

\begin{tabular}{cc|cc}
\hline \multicolumn{2}{c|}{ Right Lane } & \multicolumn{2}{c}{ Left Lane } \\
\hline Station & Marking & Station & Marking \\
\hline $10+000$ & $\mathrm{P}$ & $17+000$ & $\mathrm{~N}$ \\
$10+050$ & $\mathrm{~N}$ & $15+700$ & $\mathrm{P}$ \\
$16+02+$ & $\mathrm{P}$ & $15+286$ & $\mathrm{~N}$ \\
$16+532$ & $\mathrm{~N}$ & $10+460$ & $\mathrm{P}$ \\
$17+000$ & & $10+000$ & \\
\hline
\end{tabular}

$\mathbf{P}=$ passing zone, $\mathbf{N}=$ no-passing zone

Right and left lanes are relative to the direction of increasing stations

\subsubsection{Phase 4: Marking Using Profiles of Available and Required PSI)}

As explained in Chapter 3, there are a number of safety concerns assiciated with the marking of passing and no-passing zones according to MUTCD slandards. First, the current standards fail to consider the effect of the traffic characteristics on the minimum required PSD. A 300-m PSD is set for the 90-kph speed limit regardess of the vehicles' acceleration and deceleration capabilities or length. On the other hand, the revised model. presented in Chapter 3, can consider these traffic characteristics. For a passenger car passing a passenger car (both are $5 \mathrm{~m}$ long) and for the acceleration and deceleration capabilities discussed in Chapter 3, the required PSD is $398.9 \mathrm{~m}$. Second, the same argument is valid for the minimum length of passing zones. For the same assumptions for PSD, the minimum length of passing zones is $203.3 \mathrm{~m}$ compared to the 280 - $\mathrm{m}$ length 
recommended in the standards. Third, according to the MUTCD standards, passing zones should end when the available PSD becomes less than the required PSD. However, since the inaximum sight distance is required some distance after the pass initiation (at the critical position), the available PSD at this position will be significantly less than the required PSD. Subsequently, a pass that begins legally near the end of a passing zone will not have a sufficient sight distance for safe manoeuvring. On the other hand, the revised model uses the profile of available and required PSD to ensure that any specific pass beginning at a passing zone will have a sufficient PSD along the entire pass.

In this phase, the profiles of 2-D and 3-D available sight distance shown in Figure 6.8 and the profile of required PSD shown in Figure 3.11 are used to establish the passing and no-passing zones. A computer program was written to automate the comparison between available and required PSD as explained in Chapter 3 (Figure 3.12). The resulting markings for 2-D and 3-D alignments, referred to as the 2-D and 3-D marking. are shown in Tables 6.5 and 6.6 , respectively.

Comparing Tables 6.5 and 6.6 shows that the 2-D and 3-D markings are almost the same. According to the 2-D marking, a total of $130 \mathrm{~m}(4.5 \%$ of the $2900-\mathrm{m}$ total length of passing zones on the segment), where passing can be achieved safely, are marked as no-passing on the right lane (this total should have been $220 \mathrm{~m}$ if the warrant for passing lane is not applicable). The corresponding length on the left lane is $100 \mathrm{~m}$ (3.2\% of the 3090-m total length of passing zones on the segment). On the other hand, just $10 \mathrm{~m}$ on the right lane $(0.3 \%$ of the length of passing zones) are marked as passing while the 3-D marking indicates that passing manoeuvres are unsafe. This last condition 
TABLE 6.5: Marking Using Profiles of Required and 2-1) Available ISD.

\begin{tabular}{|c|c|c|c|c|c|}
\hline \multicolumn{3}{|c|}{ Right Lane } & \multicolumn{3}{|c|}{ Lefi L.anc } \\
\hline \multirow[t]{2}{*}{ Station } & \multicolumn{2}{|r|}{ Marking } & \multirow[t]{2}{*}{ Station } & \multicolumn{2}{|r|}{ Marking } \\
\hline & $\mathbf{P}$ or $\mathbf{N}$ & Warrant ${ }^{a}$ & & $P$ or $N$ & Warrant" \\
\hline \multirow[t]{2}{*}{$10+000)$} & & & $17+1(x)$ & & \\
\hline & $\mathbf{N}$ & PSD & & $\mathbf{N}$ & pamsing lanke \\
\hline $10+050$ & & & $16+420$ & & \\
\hline \multirow{2}{*}{$10+640$} & $\mathbf{P}$ & $\cdots$ & $15+290$ & $\mathbf{P}$ & $\cdots$ \\
\hline & $\mathbf{N}$ & passing lane & & $\mathbf{N}$ & Psil \\
\hline \multirow{2}{*}{$10+750$} & & & $14+840$ & & \\
\hline & $\mathbf{N}$ & PSD & & P & - \\
\hline $11+370$ & $\mathbf{N}$ & passing lanc & $1++520$ & $\mathbf{N}$ & ('S1) \\
\hline \multirow[t]{2}{*}{$11+640$} & & & $14+070$ & & \\
\hline & $\mathbf{N}$ & PSD & & $\mathbf{N}$ & length of passmg corte \\
\hline $11+950$ & T & . & $13+896$ & & brulue. \\
\hline \multirow[t]{2}{*}{$12+520$} & $\mathbf{N}$ & passing lanc & $13+750$ & N & moge \\
\hline & $\mathbf{N}$ & PSD & & $\mathbf{N}$ & kength of passing come \\
\hline $1.3+000$ & $\mathbf{p}$ & & $13+6 \times 0$ & 9 & 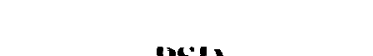 \\
\hline \multirow{2}{*}{$13+300$} & & $\cdots$ & $1.3+180$ & $\mathbf{N}$ & PSI \\
\hline & $\mathbf{N}$ & PSD & & $\mathbf{P}$ & $\ldots \ldots$ \\
\hline $13+880$ & $\mathbf{N}$ & length of passing cone & & $\mathbf{N}$ & exis \\
\hline \multirow{2}{*}{$14+1070$} & & & $12+370$ & & (1) \\
\hline & $\mathbf{N}$ & PSD & sons & $\mathbf{P}$ & .. $\cdot$ \\
\hline $14+590$ & $\mathbf{P}$ & $\ldots$ & & $\mathbf{N}$ & p'sil \\
\hline \multirow[t]{5}{*}{$16+600$} & & & $11+.340$ & & \\
\hline & & & $10+770$ & $P$ & \\
\hline & & & Cos -5 & $\mathbf{N}$ & (PSI) \\
\hline & & & & $p$ & $\ldots .$. \\
\hline & & & $1(1)+4(x)$ & & \\
\hline
\end{tabular}

$\mathbf{P}=$ passing zone, $\mathbf{N}=$ no-passing zone

Right and left ianes are relative to the direction of increasing stalions

"Warrant for no-passing zones 
TABI.E 6.6: Marking Using Profiles of Required and 3-D Available PSD.

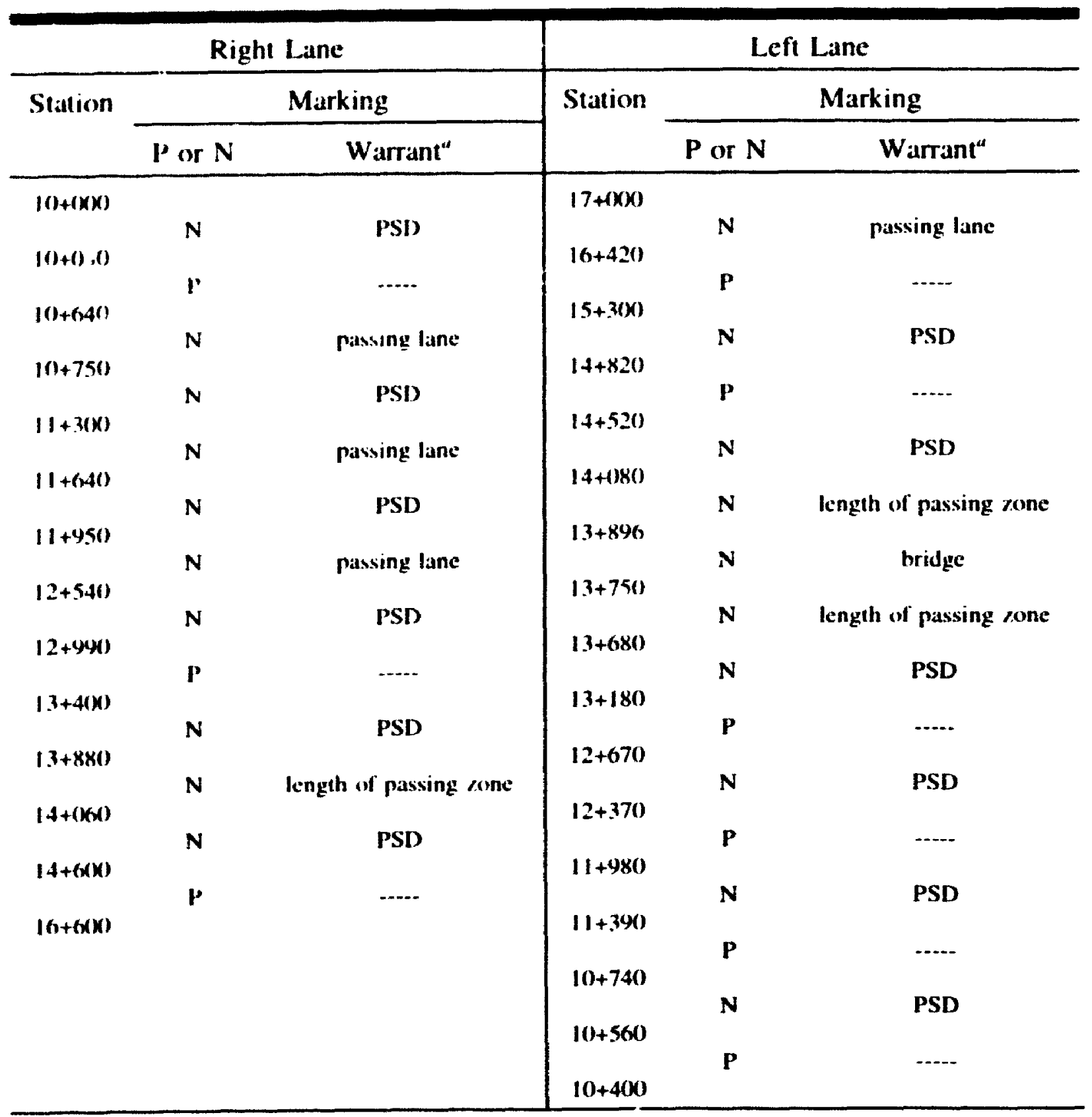

$\mathbf{P}=$ passing zone, $\mathbf{N}=$ no-passing zone

Right and left lanes are relative to the direction of increasing stations

“Warrant for no-passing zones 
is not experienced on the left lane. Subsequently. the 2-D and $\$-D$ marking dox's not yielo large difference: in the marking of passing and no-passing and. This is duc to the considerable length upon which a sufficient PSD is required. As a result, the short distances which have significant differences hetween 2-D and '-D sight disfances do mol yield large differences in the marking. In addition. as shown in Figure 6.\$, most of the differences between the 2-D and 3-D PSD are experienced at sections with available PSI) less than the required PSD.

However, a more significant difference can be seen in comparing the marking. based on the profiles of required and 3-D available PSD and the marking according th the MUTCD standards (Tables 6.6 and 6.3, respectively). As shown in the (wo tables, the MUTCD results a total of $350 \mathrm{~m}$ on the right lane $(15.0 \%$ of the $2330 \mathrm{~m}$ (otal kength of passing zones) and $1190 \mathrm{~m}(36.0 \%$ of the $3310-\mathrm{m}$ total length of passing zones) on the left lane to be marked as passing while they are unsafe according to the marking based on the profiles of required and 3-D availahle PSD. Also. $1040 \mathrm{~m}$ on the right lant and $980 \mathrm{~m}$ on the left lane $(44.6$ and $29.6 \%$ of the corresponding total length of passing zones) are marked as no-passing while they are safe according to the marking based on the profiles of required and 3-D available PSD.

Comparing Tables 6.4 and 6.6, it can be seen that the field marking has a tolal of $30 \mathrm{~m}$ (5.4\% of the $558-\mathrm{m}$ total length of passing zones) on the riglit lanc and $14 \mathrm{~m}(3.0 \%$ of the 474-m total length of passing zones) on the left lane are marked as passing although they are unsafe according to the marking based on the profiles of required and 3-D available PSD. On the other hand, $2492 \mathrm{~m}$ on the right lane and $26 \%(\mathrm{~m}$ on ahe left 
lane (446.6 and $567.5 \%$ of the corresponding total length of passing zones) are marked as no-passing while they are safe according to the marking based on the profiles of 3-D available and required PSI.

\subsection{Summary}

It is shown in the 4-phase study presented in this chapter that the 2-D and 3-D computer programs, MARKS and MiRKC, can determine the profile of available sight distance on real highway segments with complex alignments and variable side-slopes. The programs use the data that are already available in the highway agencies, and can reduce the time required to evaluate the sight distance significantly. In addition, using these computer programs reduces the cost. avoids human errors. and provides high flexibility to change the alignment and evaluate the resulting sight distance and the gains or losses in passing zones. Moreover, the decision for marking passing and no-passing zones is transferred to the engineers instead of the technicians in the field crew. However, when using the computer programs, users must be aware of the trade-offs between the computer run-time and the accuracy where both increase as the element size decreases. Generally, the element size can be set as large as $50 \mathrm{~m}$ if the alignment and the cross-section data are regular. As the irregularity in the side-slopes increases, smaller elements should be use. Comparing the 2-D and 3-D sight distances shows that differences can be significant. Ignoring the 3-D nature of highway alignments may overestimate or underestimate the available sight distance. However, because of other warrants in the marking of passing and no-passing zones, the resulting marking using 2-D ..ad 3-D 
alignments would the close. and most of the differences are in the conservative side. (Mn the other hand. comparing the marking according (1) MIITCD) standards and that hased

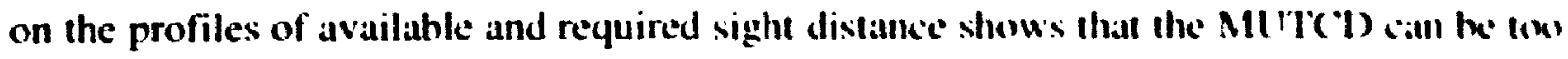
conservative or too liberal. 


\section{CHAPTER 7}

\section{ESTABLISHMENT OF DESIGN PROVISIONS}

\section{FOR 3-D HIGHWAY ALIGNMENTS}

In this chapter the three cases in which the 2-D and 3-D sight distances are significantly different are examined in more detail. As mentioned in Chapter 6, these cases are: (1) horizontal curve combined with crest vertical curve in cut section, (2) horizontal curve combined with sag vertical curve in cut section, and (3) horizontal curve combined with crest vertical curve in fill section. The model for 3-D available sight distance, presented in Chapter 5, is used to design combined horizontal and vertical alignments in cut and fill sections, and to examine the effect of considering the 3-D combined alignment on two highway design elements: namely the minimum radius of horizontal curves and the minimum length of crest verti al curves (Hassan et al, 1996d; 1996e).

\subsection{Current and 3-D Design Practices}

The current highway geometric design practice is explained in detail in Chapter 2. According to this design practice, the main design element of horizontal curves is the radius, $R$, which is determined so as to satisfy vehicle stability, driver comfort, and sight distance. The minimum radius required for vehicle stability and driver comfort can be determined using Equation 2.10 while the minimum radius required for sight distance can be determined using Equation 2.5. This latter radius should enable a driver with an $h_{1}$ eye height to see ahead an object of an $h_{2}$-height at a minimum of stopping sight distance 
(SSD). Since the design requirements are based on SSD rather than PSD. $h_{z}$ in this case is taken as $0.38 \mathrm{~m}$ (TAC. 1986). However. $h_{1}$ reconmended for design is still equal to that recommended for marking $(1.05 \mathrm{~m})$. Because the lateral clearance on a curve in a cut section varies with the height. the average height of $h_{1}$ and $h_{2}$ can the used (o) calculate the lateral clearance (AASHTO 1994). For a crest or sag vertical curve, the main design element is the curve length. $L$. or the length of vertical curve per percent change in grade. $K$ : where $K=L A$. and $A$ is the algebraic difference of the curve grades in percellt. The design values of $K$ are given in the design guides to satisfy two critcrial sght distamce and drainage (AASHTO 1994: TAC 1986). Also, when the sight distance is the governing criterion, the formulas in Equations 2.6 through 2.9 can the used.

As an alternative, the analytical model developed in this rescarch and presented in Chapter 5 can be used to design combined alignments in $1-D$ projections. The software MARKC can be used iteratively to determine the minimum requires " of a horizontal curve or $L$ of a vertical crest curve to satisfy a specific SSD. Itelative loxpss have been added to MARKC to automate the calculation of minimum $R$ or $L$. It visould be noted. however, that the calculated $R$ or $L$ will satisfy only the sight distance, and other checks for other design bases are required. Nonetheless, only the reyuired value for sight distance is used for a clear comparison between 2-D and 3-D designs.

\subsection{Study Procedure}

A horizontal circular curve combined with a vertical sag or crest curve is assumed as a typical combined alignment. The horizontal and vertical curves are posituned $x$ that the 
station of the midpoint of both curves coincide with each other. Long tangents are added at the end of either curve. Two distinct highway sections are considered: cut and fill. The design criteria and the parameters affecting the design may vary for either section as followe.

\subsubsection{Cut Section}

A highway cut section can be experienced if the natural ground level is higher than the level of the highway surface. Thus, an upward side-slope is used as a transition between the two levels. The slope, $S S$, is usually expressed as a ratio of horizontal to vertical and depends mainly on the stability of the side-slope soil (Figure 7.1). A vertical side-slope $(S S=0: 1)$ can be used if the soil is very stable, e.g. rock. Generally, cut side-slopes represent continuous lateral obstructions which may limit the available sight distance. As a result, sight distance is a controlling criterion in the design of horizontal curves. The procedure used to study the effect of 3-D consideration on the design elements can be summarized as follows:

1. A base case is used as a reference to study the effect of each element. The parameters of the base case are as follows:

- The highway is a two-lane facility which represents the dominant component of the Canadian highway network.

- The design speed, $V$, is equal to $110 \mathrm{~km} / \mathrm{h}$, which is the design speed of most rural arterial undivided highways in Canada, and the corresponding required SSD is 220 m (TAC 1986). 


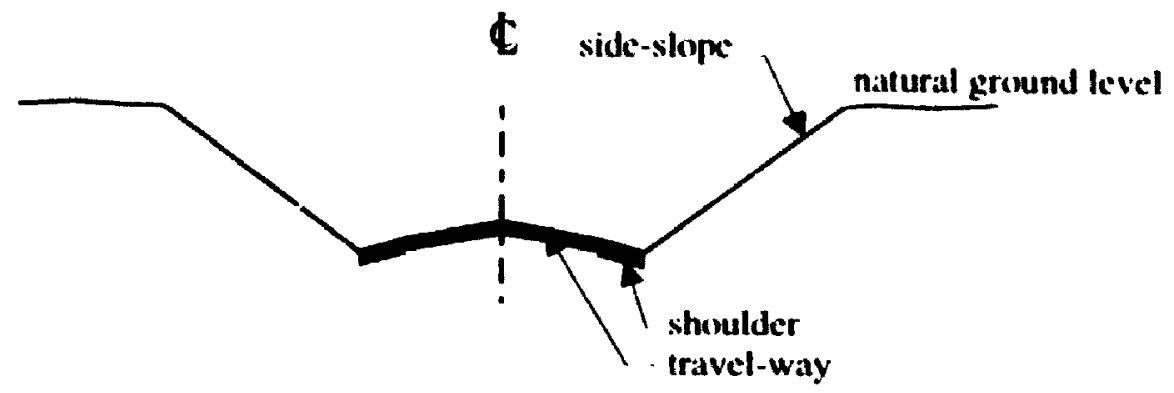

(a) Cut Section with No Down-Slope or Ditch.

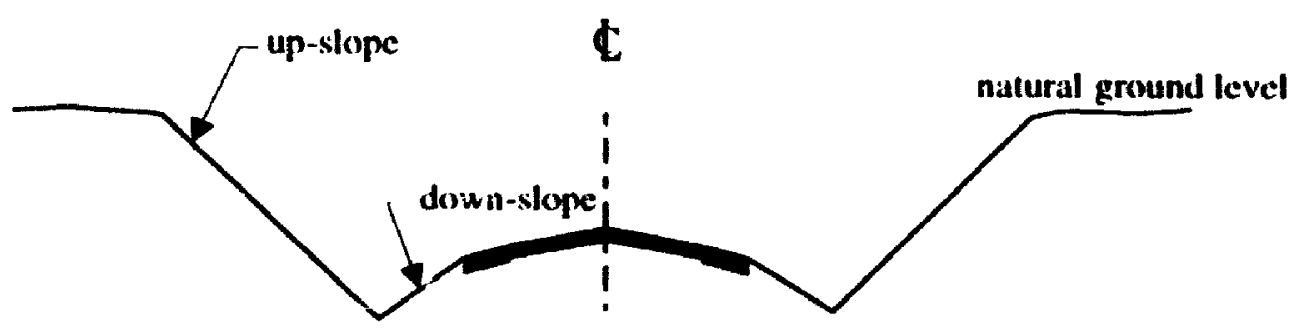

(b) Cut Section with Down-Slope.

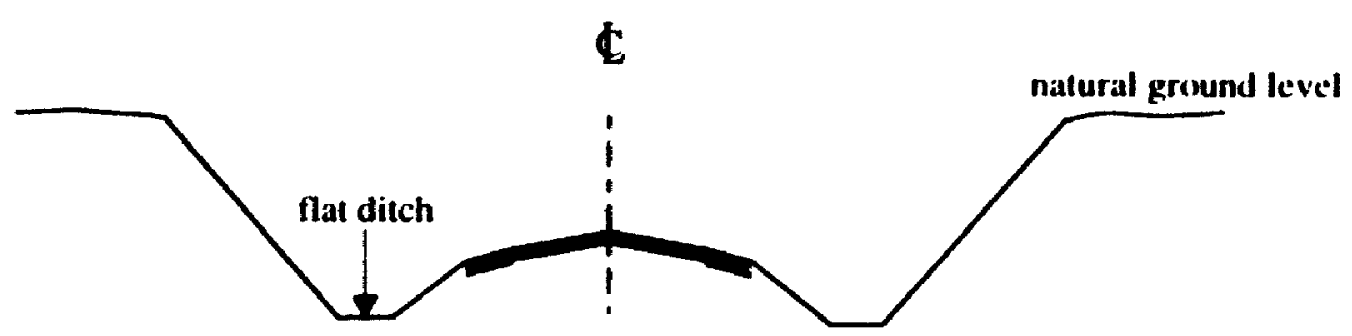

(c) Cut Section with Down-Slope and Ditch.

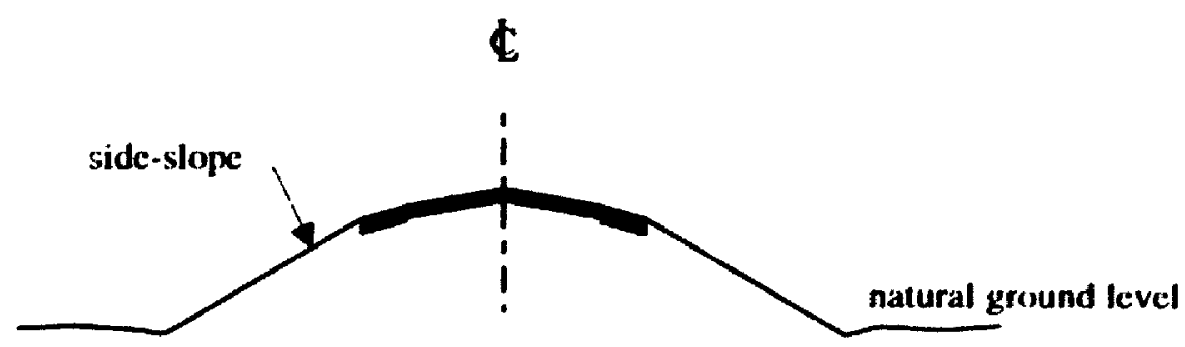

(d) Fill Section.

FIGURE 7.1: Highway Cut and Fill Sections. 
- Lane and shoulder widths are 3.7 and $2.5 \mathrm{~m}$, respectively (TAC 1986).

- The development of the superelevation is ignored; that is the entire alignment has a fully superelevated cross-section with a superelevation rate, $e$, of $6 \%$.

- The shoulder cross-slope is equal to the pavement cross-slope.

- The side-slope, SS, is 2:1. The cut side-slope begins immediately at the end of the shoulder with no down-slope or drainage ditch.

- The horizontal curve is combined with either a crest or sag vertical curve.

- The algebraic difference of the vertical curve is variable. The grades of the two tangents have the same absolute value but different signs.

- Driver's eye height, $h_{1}$, and object height, $h_{2}$, are 1.05 and $0.38 \mathrm{~m}$, respectively (TAC 1986). Thus, the lateral clearance, $m$, for current design practice is corresponding to an average height, $h_{u \text {, }}$, of $0.715 \mathrm{~m}$.

2. The required $R$ of the horizontal curve and $K$ of the vertical curve are determined according to the conventional design practice. These values will be referred to as the 2-D values. Then, due to the fact that both $R$ and $K$ depend on the sight distance, one of them, $K$, is designed conventionally and the other, $R$, is determined considering the 3-D interaction among design elements. This value will be referred to as 3-D $R$. In both cases, the object and the driver eye are positioned along the centerline of the inside lane.

3. The effect of each of the design elements on the required $R$ is studied by changing the value of one parameter at a time, while other parameters have the base values. Namely: 
- The range of $V$ is 110 and $130 \mathrm{~km} / \mathrm{h}$. The corresponding values of SSD are 220 and $260 \mathrm{~m}$, respectively (TAC 1986).

- The range of $e$ is 6 and $8 \%$.

- The range of $S S$ is $2: 1$ and $3: 1$.

- The range of the algebraic difference of the vertical curve, $A$, is 2,4 , and $6 \%$ crest and sag. A seventh case corresponds to a separate horizontal curve, i.e. $A=0$, is alsol considered.

- An additional value for $K$ is taken greater than the minimum value in the design standards.

- The range of the deflection angle of the horizontal curve, $\Delta$. begins at $5^{\prime \prime}$ and increases with a $5^{\circ}$-increment until any further increase in $\Delta$ will not change the required $\boldsymbol{R}$.

- A 1-m down-slope of $S S=2: 1$, with and without I-Im flat ditch, is added before the up-slope to help as a drainage ditch (Figure 7.1).

- The superelevation is developed over $90-\mathrm{m}$ superelevation runoff with and without spiral curve. In this case, the normal crown slope, $q$, is $2 \%$.

\subsubsection{Fill Section}

If the natural ground level at the location of the highway is lower than the required level of the highway surface, a fill soil is used to reach the required levels forming a fill section (Figure 7.1). In this case, no lateral obstruction exists, and therufore horizontal sight distance is not a governing criterion for $R$, but $K$ is still controlled by the sight 
distance. The study approach is similar to that for the cut sections but with some differences:

1. The side-slope, $S S$, is not a parameter because it does not affect either $R$ or $K$.

2. $R$ is designed based on the stability condition only (Equation 2.10 ). Then, $K$ is determined according to the current practice and considering the effect of the 3-D interaction.

3. The effect of the change in each parameter is studied with respect to the required 3-D $\boldsymbol{K}$.

4. Both the inside and outside lanes are studied. The driver's eye and the object are positioned along the centerline of the lane being considered.

\subsection{Results and Analysis}

The design values based on the current design practice are shown in Table 7.1. In this table, a half of the lane width is added to the values of $\boldsymbol{R}$ for the sight distance calculated using Equation 2.5 because the equation gives the radius of the centerline of the inside lane while the design values are always expressed in terms of the radius of the highway centerline. These values are used as reference for studying the effect of 3-D consideration.

\subsubsection{Cut Seations}

As mentioned previously, the length of the vertical curve, $L$, in this case is taken as the 2-D value, that is $L=K A$, where the values of $K$ are shown in Table 7.1. Then, the 
TABLE 7.1: Minimum Horizontal Curve Radius, $R$, and Design Values of $\boldsymbol{K}$ According to Current Design Practice.

\begin{tabular}{|c|c|c|c|c|c|c|}
\hline \multirow{2}{*}{$\begin{array}{c}V \\
(\mathrm{~km} / \mathrm{h})\end{array}$} & \multirow{2}{*}{$\begin{array}{c}\text { SSD }^{a} \\
(\mathbf{m})\end{array}$} & \multirow{2}{*}{$f_{\mathrm{s}}{ }^{a}$} & \multicolumn{2}{|c|}{$R(\mathrm{~m})^{h}$} & \multicolumn{2}{|c|}{$\boldsymbol{K}(\mathrm{II})$ “ } \\
\hline & & & $\begin{array}{c}\text { Vehicle } \\
\text { Stability }\end{array}$ & $\begin{array}{c}\text { Sight } \\
\text { Distunce }\end{array}$ & $\begin{array}{l}\text { Crest } \\
\text { Curve }\end{array}$ & $\begin{array}{l}\text { Sug } \\
\text { Curve }\end{array}$ \\
\hline 110 & 220 & 0.10 & 595.5 & 960.9 & 85 & 55 \\
\hline 130 & 260 & 0.08 & 950.5 & 1341.7 & 120 & 6.5 \\
\hline
\end{tabular}

“ From (TAC, 1986)

b Alignment parameters have the base values

- For fill section only. The lateral clearance, $m$, is equal to $6.302 \mathrm{~m}$ (corresponding 10 an average height of $0.715 \mathrm{~m}$ )

minimum 3-D $R$ is determined by iterations to the nearest $0.1 \mathrm{~m}$ to satisfy the sight distance condition. It should be noted that these values should not be less than the minimum value for vehicle stability shown in Table 7.1. However, as mentioned earlier. to have a clear comparison with the 2-D values, only the values controlled by the sight distance are shown. Table 7.2 shows the percentage difference between the 2-1) and 3-I) radii, Diff, that is calculated as:

$$
\text { Diff }=\frac{(3-D \text { value })-(2-D \text { value })}{(2-D \text { value })} \times 100
$$

As shown in the equation, the 2-D values are taken as the reference for comparison because designers and engineers are more familiar with them. Thus, a negative value of Diff indicates that the 2-D analysis overestimates the design, while a positive value indicates that the 2-D analysis underestimates the design. 
TABLE 7.2: Difference Between 2-D and 3-D Radii for Horizontal Curves Combined with Vertical Curve.

\begin{tabular}{|c|c|c|c|c|c|c|c|}
\hline \multirow{3}{*}{$\Delta$} & \multicolumn{7}{|c|}{ Diff (\%) } \\
\hline & \multicolumn{3}{|c|}{$A$ (crest curves) } & \multirow{2}{*}{$A=0$} & \multicolumn{3}{|c|}{$A$ (sag curves) } \\
\hline & $6 \%$ & $4 \%$ & $2 \%$ & & $2 \%$ & $4 \%$ & $6 \%$ \\
\hline \multicolumn{8}{|c|}{ (a) $V=110 \mathrm{~km} / \mathrm{h}(S S D=220 \mathrm{~m}) .2-\mathrm{D} R=960.9 \mathrm{~m}$. } \\
\hline 5 & $-100.00(0)$ & $-100.00(0)$ & $-100.00(0)$ & $-100.00(0)$ & $-100.00(0)$ & $-100.00(0)$ & $-100.00(0)$ \\
\hline 10 & $29.84(218)$ & $29.84(218)$ & $27.72(214)$ & $.9 .26(152)$ & $-54.56(76)$ & $-69.67(51)$ & $-69.76(51)$ \\
\hline 15 & $29.88(327)$ & $29.88(327)$ & $27.90(322)$ & $0.35(253)$ & $-21.47(198)$ & $-28.25(181)$ & $-28.25(181)$ \\
\hline 20 & & & & & $-16.00(282)$ & $-25.56(250)$ & $-25.82(249)$ \\
\hline 25 & & & & & $-5.67(396)$ & $-22.39(325)$ & $-25.82(311)$ \\
\hline 30 & & & & & $-0.30(502)$ & $-9.95(453)$ & $-25.16(377)$ \\
\hline 35 & & & & & $0.35(589)$ & $-1.36(579)$ & $-18.21(480)$ \\
\hline 40 & & & & & & $0.36(673)$ & $-3.48(648)$ \\
\hline 45 & & & & & & & $0.32(757)$ \\
\hline 50 & & & & & & & $0.37(842)$ \\
\hline \multicolumn{8}{|c|}{ (b) $V=130 \mathrm{~km} / \mathrm{h}(S S D=260 \mathrm{~m}) .2-\mathrm{D} R=1341.7 \mathrm{~m}$. } \\
\hline 5 & $-37.47(73)$ & $-37.47(73)$ & $-38.32(72)$ & $-100.00(0)$ & $-100.00(0)$ & $-100.00(0)$ & $-100.00(0)$ \\
\hline 10) & $29.36(303)$ & $29.36(303)$ & $29.13(302)$ & $-0.84(232)$ & $-39.17(143)$ & $-51.99(112)$ & $-52.02(112)$ \\
\hline 15 & & & & $0.31(352)$ & $-23.17(270)$ & $-29.31(248)$ & $-29.31(248)$ \\
\hline 20 & & & & & $-9.87(422)$ & $-27.65(339)$ & $-29.12(332)$ \\
\hline 25 & & & & & $-0.69(581)$ & $-14.15(503)$ & $-28.99(416)$ \\
\hline 30 & & & & & $0.32(705)$ & $-1.24(694)$ & $-22.87(542)$ \\
\hline 35 & & & & & & $0.33(822)$ & $-2.12(802)$ \\
\hline 40 & & & & & & & $0.34(940)$ \\
\hline
\end{tabular}

- $c=6 \% . S S=2: 1, K=85 \mathrm{~m}$ (crest), $K=55 \mathrm{~m}$ (sag)

- Values between parentheses are the length of horizontal curve, $L_{c}$, in meters

- Below the solid line, further increases in $\Delta$ do not change the value of Diff 
As shown in the table, three possibilities exist for the combined alignment. First, if the horizontal curve is combined with a crest vertical curve. the 3-D $R$ is less than the 2-D value for small angles of deflection. $\Delta$, where $R$ can vanish indicating that the sight distance is not a controlling criterion. However. for larger $\Delta$. the required 3-D $R$ is greater than the required 2-D value. The reason for this increase is that the curvature of the crest curve reduces the effective $h_{a v}$ at which the sight line is tangent to the side-slope. This in turn reduces the lateral clearance, $m$, compared to the clearance at the average height of $h_{1}$ and $h_{2}$. The reduction in $m$ is maximum when the vertical curve length, $l$. is equal to or greater than SSD. Therefore, the required 3-D $R$ will not depend on $L$. and in turn on $A$. This can be observed by comparing Diff for $A=4$ and $6 \%$, where for SSI) $=220 \mathrm{~m}, L$ is 340 and $510 \mathrm{~m}$, respectively, and for $S S D=260 \mathrm{~m}, L$ is 480 and $720 \mathrm{~m}$. respectively. However, if $L$ is less than SSD. as $A$ and $L$ decrease, the reductions in $m$ and $h_{a r}$ decrease, and thus the required 3-D $R$ decreases. This trend can be observed by comparing Diff for $A=2$ and 4, where for $\operatorname{SSD}=220 \mathrm{~m}, L$ is 170 and $340 \mathrm{~m}$, respectively, and for SSD $=260 \mathrm{~m}, L$ is 240 and $480 \mathrm{~m}$, respectively. This trend continues until $A$ vanishes, that is a case of no vertical curve, where the 3-D and 2-D values of $R$ are basically the same. For the same value of $A$, the difference between the 3-D and 2-D values of $R$ increases as $\Delta$ increases until the length of the horizontal curve is equal to SSD. This can be seen in the stabilization of Diff when the horizontal curve length, $L_{t}$, is greater than or equal to SSD.

Second, if a sag curve is combined with the horizontal curve, the effective $h_{a v}$ at which the sight line is tangent to the side-slope increases. Therefore, as shown in the 
table, the 3-D $R$ is less than the corresponding 2-D value. Also, the larger the $A$, the greater the difference hetween the 3-D and the 2-D values. For the same $A$, as $\Delta$ increases the 3-D $R$ increases. However, unlike the case of a crest curve, the change of the 3-D $R$ continues even after the length of the horizontal curve is greater than SSD. However, if $L \geq \operatorname{SSD}$ and $L \geq L_{1}$, any further increase in $A$ and $L$ will not affect the required 3-D $R$. For example, for SSD $=220 \mathrm{~m}$, Diff for $A=4 \%(L=220 \mathrm{~m})$ is equal to Diff for $A=6 \%(L=330 \mathrm{~m})$ up to $\Delta=15^{\circ}\left(L_{\mathrm{c}}=180.5 \mathrm{~m}\right)$. Similarly, for SSD $=260$ m. Diff for $A=4 \%(L=260 \mathrm{~m})$ is equal to Diff for $A=6 \%(L=390 \mathrm{~m})$ up to $\Delta=15^{\circ}$ $\left(L_{1}=248.3 \mathrm{~m}\right)$. The reason for this is that the critical part of the curve is not the part combined with the vertical curve: but rather each of the two parts with no overlap with the vertical curve at both ends. When either of these parts equals SSD, this part will act as a separate horizontal alignment and will control the 3-D $R$. Therefore, $R$ is practically equal to the value corresponding to $A=0$, and any further increase in $\Delta$, and in turn in the length of the horizontal curve, has no effect on the 3-D $R$.

The same trend in both cases of crest and sag curves is valid regardless of the design speed. However, for the same $\Delta$ of the horizontal curve, the higher the design speed, the higher the effect of crest curves and the lower the cffect of sag curves. For example, when $\Delta=10^{\circ}$ and $A=2 \%$, as the design speed increases from 110 to $130 \mathrm{~km} / \mathrm{h}$, the difference between the 2-D and 3-D values of $R$ increases from 27.72 to $29.13 \%$ for a horizontal curve combined with a crest curve while the difference decreases from $\mathbf{5 4 . 5 6}$ to $39.17 \%$ for a horizontal curve combined with a sag curve. Also, as the design speed increases, the value of $\Delta$ beyond which it has no effect decreases. For example, when 
$A=2 \%$ and $V=110 \mathrm{~km} / \mathrm{h}$, the value of $\Delta$ has no effect beyond $15^{\circ}$ for a horizontal curve combined with a crest curve and $45^{\circ}$ for a horizontal curve combined with a sag curve while the corresponding values when $V=130 \mathrm{~km} / \mathrm{h}$ are $10^{\circ}$ and $30^{\circ}$. respectively.

Third, if no vertical curve exists. i.e.. $A=0$, the 3-D $R$ for short horizontal curves. small $\Delta$, is less than the 2-D $R$. As $\Delta$ and the length of the horizontal curve increase, Equation 2.5 will be more accurate, and therefore the difference between the 3-D and 2-1) values of $R$ decreases. This trend continues until the length of the horizontal curve is equal to SSD. However, as shown in the table, for horizontal curves longer than SSD, a slight difference between the 2-D and 3-D radii still exists. This is due to the fact that both the lateral obstruction by the side-slope and the sight line are sloped in the same direction (the sight line is sloped from a $1.05 \mathrm{~m}$ driver eye $(1)$ a $0.38 \mathrm{~m}$ object). As a result, the point of tangency between the sight line and the obstruction is not at $h_{a}$ exactly. Such difference is expected to decrease as SS decreases until it vanishes when $S S$ is equal to $0: 1$ (vertical obstruction). To verify this hypothesis, Table 7.3 shows the 2-D and 3-D radii for separate horizontal curves with lengths longer than SSD using different values of $S S(A=0)$. As shown in the table, for a vertical side-slope, $S S=0: 1$, the difference between the 2-D and 3-D radii is $0.1 \mathrm{~m}$ which is the accuracy used 10 determine $R$. As $S S$ increases, Diff increases. Yet, the difference is very minimal (less than $0.5 \%$ ).

The effect of the remaining parameters on the difference between the 2-D and 3-D values of $R$ can be seen by comparing Table $7.2 a$ with Table 7.4. In cach part of Table 7.4, all the parameters have the base values and only one parameter is changed. 


\begin{tabular}{|c|c|c|c|c|c|}
\hline \multirow{2}{*}{ SS } & \multicolumn{2}{|c|}{ 2-D } & \multirow{2}{*}{$\begin{array}{c}3 \mathrm{D} R \\
(\mathrm{~m})\end{array}$} & \multirow{2}{*}{$\begin{array}{l}\text { Difference } \\
\text { (m) }\end{array}$} & \multirow{2}{*}{$\begin{array}{l}\text { Diff } \\
(\%)\end{array}$} \\
\hline & $m(\mathbf{m})$ & $R(\mathrm{~m})$ & & & \\
\hline ():1 & 4.350 & 1392.0 & 1392.1 & 0.1 & 0.0072 \\
\hline $1: 1$ & 5.326 & 1136.9 & 1138.5 & 1.6 & 0.1405 \\
\hline $2: 1$ & 6.302 & 960.9 & 964.3 & 3.4 & 0.3526 \\
\hline 3:1 & 7.278 & 832.0 & 836.0 & 4.0 & 0.4785 \\
\hline
\end{tabular}

Comparing the values of Diff in Tables $7.2 a$ and $7.4 a$ shows that for the same $\Delta$ and $A$, the effect of the combined alignment decreases as the vertical curve becomes flatter, that is $K$ hecomes larger. This finding is expected since as $K \rightarrow \infty$, the alignment will end up as a separate horizontal curve. On the other hand, Comparing Tables $7.2 a$ and $7.4 b$ shows that as the superelevation rate, $e$, increases, the effect of crest curves decreases slightly while the effect of sag curves increases slightly. The reason for this is that as $e$ increases, the difference in elevation between the centerline of the inside lane (where the driver eye and the object are positioned) and the end of the shoulder (where the side-slope begins) increases. As a result, the effective height at the point of tangency between the sight line and the side-slope increases, and in turn the effective lateral clearance increases. This will reduce the increase of $R$ on crest curves and will increase the reduction of $R$ on sag curves. Comparing Tables $7.2 a$ and $7.4 c$, it is shown that as $S S$ increases, the effect of both crest and sag curves increases. This is due to the fact that for flatter slopes (higher SS). the difference in the effective $h_{u r}$ will result in higher difference in the lateral 
TABLE 7.4: Effect of Vertical Curvature, Superelevation Rate, and Side-Slope on 3-D $R$.

\begin{tabular}{|c|c|c|c|c|c|c|c|}
\hline \multirow{3}{*}{$\Delta$} & \multicolumn{7}{|c|}{ Diff $(\%)$} \\
\hline & \multicolumn{3}{|c|}{$A$ (crest curves) } & \multirow{2}{*}{$A=0$} & \multicolumn{3}{|c|}{ A (saly curves) } \\
\hline & $6 \%$ & $4 \%$ & $2 \%$ & & $2 \%$ & $w$ & $6 \%$ \\
\hline \multicolumn{8}{|c|}{ (a) $K$ (crest) $=102$ and $K($ sag $)=66.2-D R=9(0) .9 \mathrm{~m}$. } \\
\hline 10 & 23.31 & 23.31 & 23.12 & -9.26 & -.51 .59 & -59.65 & -59.65 \\
\hline 20 & 23.79 & 23.79 & 23.62 & 0.35 & -16.12 & -22.44 & -22.44 \\
\hline 30 & & & & & -0.88 & -14.54 & -22.43 \\
\hline 40 & & & & & 0.36 & 0.11 & -11.80 \\
\hline 50 & & & & & & 0.36 & 0.37 \\
\hline \multicolumn{8}{|c|}{ (b) Superelevation rate, e. $=8 \%, 2-D R=935.1 \mathrm{~m}$. } \\
\hline 10 & 28.52 & 28.52 & 26.38 & -11.68 & -58.21 & -73.77 & -73.84 \\
\hline 20 & 28.82 & 28.82 & 26.92 & 0.32 & -16.73 & -25.1 .5 & -2.5 .32 \\
\hline 30 & & & & & -0.75 & -11.68 & $-24 .(x)$ \\
\hline 40 & & & & & 0.33 & 0.32 & -5.35 \\
\hline 50 & & & & & & 0.34 & 0.34 \\
\hline \multicolumn{8}{|c|}{ (c) $S S=3: 1 . \quad 2-\mathrm{D} R=832.0 \mathrm{~m}$. } \\
\hline 10 & 42.20 & 42.20 & 38.69 & -25.55 & $-1(0) .00$ & $-\mid(x) .(x)$ & $-I(X) .(M)$ \\
\hline 20 & 42.79 & 42.79 & 39.69 & 0.48 & -25.14 & -31.88 & -31.88 \\
\hline 30 & & & & & -5.28 & -27.49 & .31 .11 \\
\hline 40 & & & & & 0.50 & -2.12 & $-2 x .10$ \\
\hline 50 & & & & & & 0.49 & .0 .12 \\
\hline 60 & & & & & & & (1.49) \\
\hline
\end{tabular}

Below the solid line, further increases in $\Delta$ do not change the value of Diff 
clearance, $m$. and in turn higher effect of the combined alignment. If the lateral obstruction is vertical (SS=0):1), the change in the effective $h_{a 1}$ will not cause any difference in $m$, and thus the combined alignment will have no effect.

Table 7.5 a shows the effect of adding a 1-m down-slope $(S S=2: 1)$ without flat ditch before the up-slope. and Table 7.5b shows the effect of the same down-slope with a $1-\mathrm{m}$ llat ditch (Figure 7.1 ). In the first case, the lateral clearance. $m$. at $h_{\omega}$ is $8.302 \mathrm{~m}$ and the required 2-D $R$ is $729.3 \mathrm{~m}$. When the flat ditch is added, $m$ increases to 9.302 $\mathrm{m}$ and the required 2-D $R$ decreases to $650.7 \mathrm{~m}$. Comparing Tables $7.2 a$ and 7.5 shows that the same trends discussed in the base case are sti!! ; aiid. However, the effect of the crest curve on the required 3-D $R$ is reduced by the addition of the down-slope. and is reduced even more by the addition of the flat ditch. For example, for $A=6 \%$. the corresponding maximum value of Diff in the base case is $29.88 \%$. compared to $21.10 \%$ when the down-slope is added. This maximum value of Diff is further reduced to $18.52 \%$ when the flat ditch is added. This reduction in the effect of the crest curve can be due to the increase of the 2-D lateral clearance, $m$, at $h_{\omega}$ while the absolute decrease in the 3-D $m$ due to the vertical curvature remains the same. As a result, the decrease in the 3D $m$ will represent a lower percentage and will have a lower effect on the 3-D $R$ than that in the base case. In case of sag vertical curves, the same effect is noted for the large values of $\Delta$. For example, for $A=6 \%$ and $\Delta=30^{\circ}$. Diff decreases from $-25.16 \%$ in the base case to -20.94\% when the down-slope is added. The corresponding value of Diff decreases more to $-19.16 \%$ when the flat ditch is added. However, for small values of $\Delta$. both the down-slope and the ditch increase the effect of the sag curve. This can be 
TABLE 7.5: Efrect of Down-Slope and Drainage Ditch Before ('p-Slope on 3-1) $\mathrm{k}$.

\begin{tabular}{|c|c|c|c|c|c|c|c|}
\hline \multirow{3}{*}{$\Delta$} & \multicolumn{7}{|c|}{ lNiff $(\because)$} \\
\hline & \multicolumn{3}{|c|}{ A (crest curves) } & \multirow{2}{*}{$A=0$} & \multicolumn{3}{|c|}{ A (rigk cullow) } \\
\hline & $6 \%$ & $4 \%$ & $2 \%$ & & $\because \because$ & H'i & ti: \\
\hline \multicolumn{8}{|c|}{ (a) Down-slope before the up-slope. 2-D $R=730.3 \mathrm{~m}$. } \\
\hline 10 & -1.23 & -1.23 & -3.92 & -.52 .86 & $-1(x)(x)$ & $\ln (\mathrm{x}) \mathrm{(x)}$ & $-1(x)(x)$ \\
\hline 20 & 21.10 & 21.10 & 19.79 & (1).11) & -16.66 & -21.64 & $21 .(14)$ \\
\hline 30 & & & & & -7.54 & $-19 .(x)$ & $20)(1.4$ \\
\hline 40 & & & & & $-(0.21$ & o.tod & $11) .4$ \\
\hline 50 & & & & & 021 & $(i, 1$, & 550 \\
\hline 60 & & & & & & 0.21 & 11.21 \\
\hline
\end{tabular}

(b) Down-slope and ditch before the up-slope. $2-1) \mathrm{K}=6.50 .7 \mathrm{~m}$.

\begin{tabular}{|c|c|c|c|c|c|c|}
\hline-29.86 & -29.86 & -32.80 & -87.51 & $-1(0) .(0)$ & $-1(x),(x)$ & $f(x)(x)$ \\
\hline 18.52 & 18.52 & 17.37 & 0.2 .3 & -16.74 & -22.44 & 22.44 \\
\hline 30 & & & & -10.36 & $-1 \times .73$ & 16.16 \\
\hline 40 & & & & -1.87 & -11.34 & $1 \times .83$ \\
\hline 50 & & & & 0.23 & -1.80 & 12.60 \\
\hline 60 & & & & & 0.25 & 1.71 \\
\hline 70 & & & & & & 0.25 \\
\hline
\end{tabular}

- e =6\%. SS $=2: 1, K=85 \mathrm{~m}$ (crest), $K=55 \mathrm{~m}$ (sig)

- Down-slope: $S S=2: 1$, width $=1 \mathrm{~m}$. Ditch width $=1 \mathrm{~m}$

- Below the solid line, further increases in $\Delta$ do not change the value of $/$ Itfl 
seen by comparing the values of Diff for $A=6 \%$ and $\Delta=15^{\circ}$ (not shown in Table 7.5). The corresponding values for the base case, the base case with down-slope, and the base case with down-slope and ditch are $-28.25,-37.76$, and $-48.26 \%$, respectively. This is due to the fact that the large lateral clearances, $m$, when the down-slope and the ditrh are added, accompanied with small $\Delta$ reduce the required $R$ significantly, and may not require a curve at all.

Finally, Table 7.6 shows the minimum 3-D $R$ for the horizontal curve with all parameters having the base values. However, in this case, the transition from a normal crown cross-section to a fully superelevated cross-section is considered with and without a spiral curve. In either case, a $90 \mathrm{~m}$-transition is used so that the rate of change of the elevation of either the inside or the outside edges does not exceed 1:400 (TAC 1986). Figure 7.2 shows the development of the superelevation in both cases. As shown in the table, for smaller $\Delta$, the consideration of the superelevation development raises the elevations of the driver eye and the object and thus the effective average height. Subsequently, the required $R$ is reduced slightly. Therefore, the elfect of the sag curve is slightly aggravated while the effect of the crest curve is slightly reduced. However, if the horizont.1 curve is long enough so that the development of the superelevation does not interact with the vertical alignment, the required $R$ will not change whether the development is considered or not. This is reflected in the value of $R$ which is required for large $\Delta$. 
Tangent-Spiral

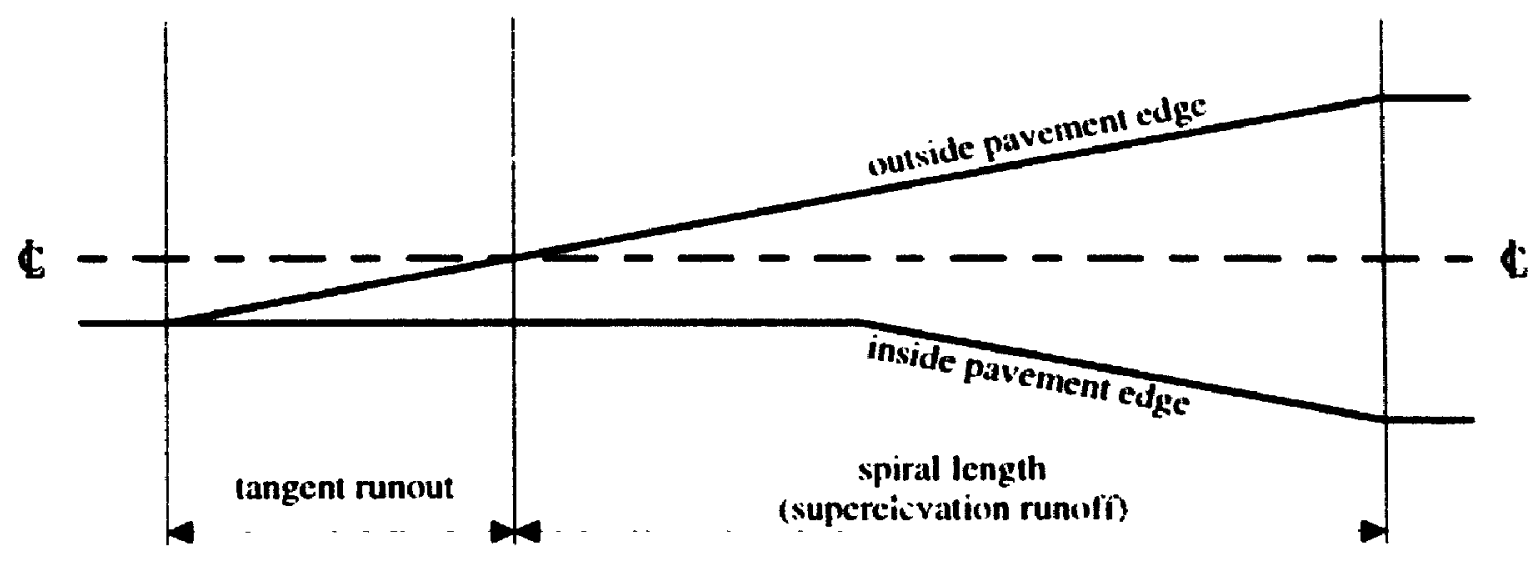

(a) Horizontal Curve with Spiral

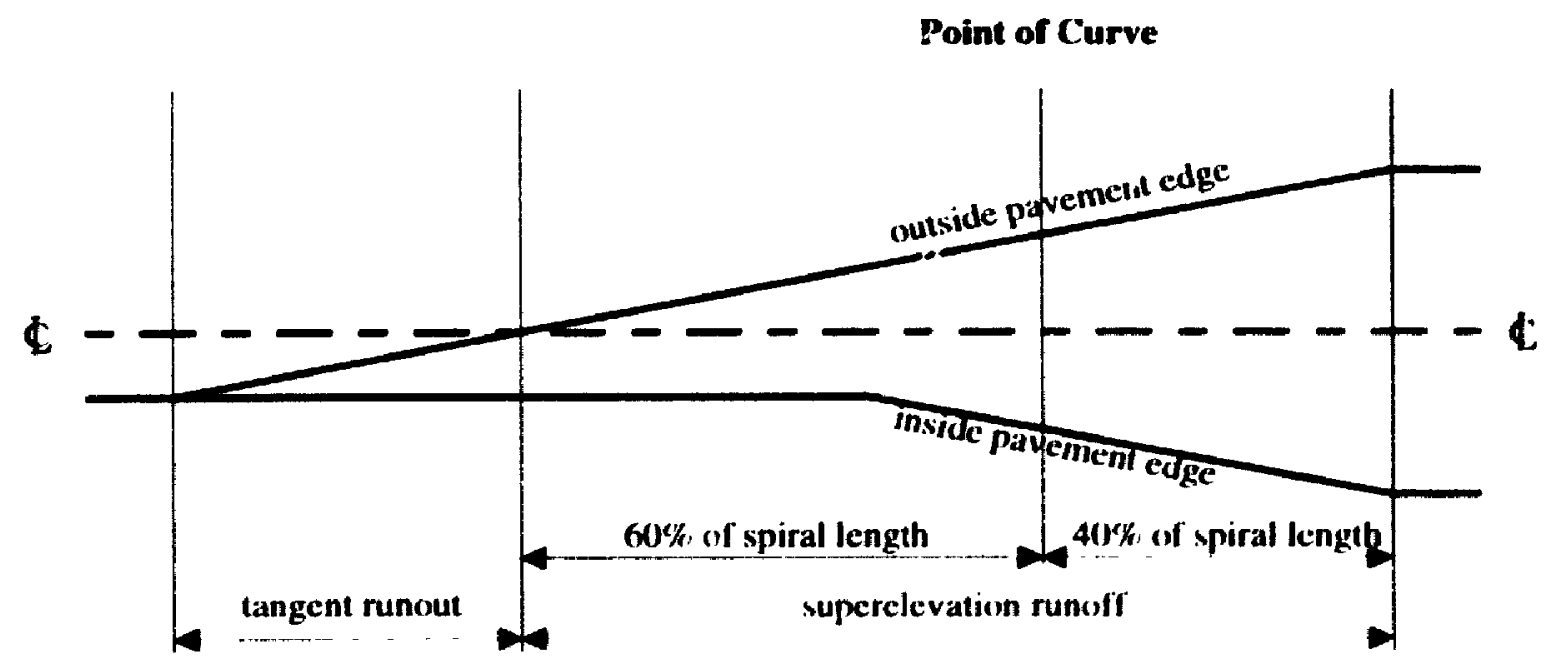

(b) Horizontal Curve without Spiral 
TABIE 7.6: Minimum $R$ for Horizontal Curve Considering the Superelevation Development.

\begin{tabular}{|c|c|c|c|c|c|c|c|}
\hline \multirow{3}{*}{$\Delta$} & \multicolumn{7}{|c|}{$R(\mathrm{~m})$} \\
\hline & \multicolumn{3}{|c|}{$A$ (crest curves) } & \multirow{2}{*}{$A=0$} & \multicolumn{3}{|c|}{$A$ (sag curves) } \\
\hline & $6 \%$ & $4 \%$ & $2 \%$ & & $2 \%$ & $4 \%$ & $6 \%$ \\
\hline \multicolumn{8}{|c|}{ (a) Curves with spiral. 2-D $R=960.9 \mathrm{~m}$. } \\
\hline 10 & 1156.6 & 1156.6 & 1130.2 & 709.8 & N/A & N/A & N/A \\
\hline 20 & 1247.9 & 1247.9 & 1229.0 & 964.3 & 758.8 & 698.4 & 698.4 \\
\hline 30 & & & & & 928.9 & 801.9 & 712.8 \\
\hline 40 & & & & & 964.3 & 952.9 & 866.3 \\
\hline 50 & & & & & & 964.4 & 963.1 \\
\hline 6) & & & & & & & 964.5 \\
\hline \multicolumn{8}{|c|}{ (b) Gradual change of cross-slope without spiral. 2-D $R=960.9 \mathrm{~m}$. } \\
\hline 10 & 1221.8 & 1221.8 & 1201.1 & 832.7 & N/A & N/A & N/A \\
\hline 20 & 1248.0 & 1248.0 & 1229.0 & 964.3 & 793.6 & 710.3 & 708.2 \\
\hline 30 & & & & & 949.0 & 847.4 & 713.5 \\
\hline 40 & & & & & 964.3 & 958.9 & 911.5 \\
\hline 50 & & & & & & 964.4 & 964.5 \\
\hline \multicolumn{8}{|c|}{$\begin{array}{l}\text { N/A = Not applicable (the length of the curve is not enough for the development of } \\
\text { the superelevation) } \\
-q=2 \%, e=6 \%, S S=2: 1, K=85 \mathrm{~m} \text { (crest), } K=55 \mathrm{~m} \text { (sag) } \\
\text { - Below the solid line, further increases in } \Delta \text { do not change the value of } R\end{array}$} \\
\hline
\end{tabular}




\subsubsection{Fill Sections}

As mentioned earlier, in this case, the main parameter is the length of the vertical curve. $L$, or the vertical curvature which is expressed in terms of, $K$. However. because the analytical model cannot consider the headlight sight distance, only crest curves are considered in this study. The range of the algebraic difference in grades, $A$, is $+1012 \%$. The radius of the horizontal curve is controlled by the vehicle stability only. Therefure. $R$ is taken as 600 and $950 \mathrm{~m}$ fur the design speeds of 110 and $130 \mathrm{~km} / \mathrm{h}$, respectively (Table 7.1). The other design elements base values are the same as those used in cut sections. However, the degree of alignment overlap is changed by increasing the length of the horizontal curve, $L_{t}$, with $100 \mathrm{~m}$-increments from zero (separate vertical alignment) until the required 3-D $K$ does not change with the change of $L_{1}$. The minimum 2-ID $K$ corresponding to design speeds of 1,0 and $130 \mathrm{~km} / \mathrm{h}$, calculated using Equations 2.6 and 2.7, are 89.9 and $125.5 \mathrm{~m}$, respectively. These values are slightly larger than those recommended in the design guides. Possibly this is due to the fact that the required SSI) shown in the table are rounded while the design values of $K$ are based on the exact SSD before approximation. Therefore, for consistency, the calculated values are used as the base of comparison with the 3-D $K$.

Table 7.7 shows the percentage difference between the 2-D and 3-D $K$ values, Diff, calculated using Equation 7.1. As shown, when a portion of the vertical curve, of a length equal to or more than SSD, is not overlapping with the horizontal curve, the 2-I) and 3-D $K$ values are practically the same. This is due to the fact that this portion will act as a separate vertical alignment and will control the required vertical curvature. 
TABLE 7.7: Difference Between 2-D and 3-D $K$ Values for Crest Vertical Curves Combined with Horizontal Curve.

\begin{tabular}{|c|c|c|c|c|c|}
\hline \multirow{2}{*}{$\begin{array}{c}L_{1} \\
(\mathrm{~m})\end{array}$} & \multicolumn{5}{|c|}{ Diff $(\%)$} \\
\hline & $A=4 \%$ & $A=6 \%$ & $A=8 \%$ & $A=10 \%$ & $A=12 \%$ \\
\hline \multicolumn{6}{|c|}{ Inside Lane } \\
\hline 0 & 0.00 & 0.00 & 0.00 & 0.00 & 0.00 \\
\hline 100 & -31.92 & 0.00 & 0.00 & 0.00 & 0.00 \\
\hline 200$)$ & -66.52 & -13.13 & 0.00 & 0.00 & 0.00 \\
\hline $3(0)$ & -71.64 & -59.96 & -0.22 & 0.00 & 0.00 \\
\hline 400 & & & -59.73 & 0.00 & 000 \\
\hline $5(X)$ & & & & -1.56 & 0.00 \\
\hline 600) & & & & -59.51 & 0.00 \\
\hline 700 & & & & & -59.29 \\
\hline \multicolumn{6}{|c|}{ Outside lane } \\
\hline () & 0.00 & 0.00 & 0.00 & 0.00 & 0.00 \\
\hline 100 & -32.15 & 0.00 & 0.00 & 0.00 & 0.00 \\
\hline 200 & -50.17 & -12.46 & 0.00 & 0.00 & 0.00 \\
\hline $3(0)$ & -.50 .83 & -48.83 & -0.22 & 0.00 & 0.00 \\
\hline $4(0)$ & & & -48.61 & 0.00 & 0.00 \\
\hline 500 & & & & -1.22 & 0.00 \\
\hline 600 & & & & -48.39 & 0.00 \\
\hline 700$)$ & & & & & .48 .16 \\
\hline \multicolumn{6}{|c|}{ Both Lanes } \\
\hline 0 & 0.00 & 0.00 & 0.00 & 0.00 & 0.00 \\
\hline 100 & -31.92 & 0.00 & 0.00 & 0.00 & 0.00 \\
\hline 200 & -50.17 & -12.46 & 0.00 & 0.00 & 0.00 \\
\hline $3(x)$ & -50.83 & -48.83 & -0.22 & 0.00 & 0.00 \\
\hline $4(0)$ & & & -48.61 & 0.00 & 0.00 \\
\hline 500 & & & & -1.22 & 0.00 \\
\hline $6(0)$ & & & & -48.39 & 0.00 \\
\hline 700 & & & & & -48.16 \\
\hline
\end{tabular}

2-D $K=89.9 \mathrm{~m}$

Below the solid line, further increases in $L_{c}$ do not change the value of Diff 
However, when the entire vertical curve is combined with the horisontitl curve, (he 3.1) $K$ is reduced significantly relative to the 2-D $K$. The reduction maly be ats high as $70 \%$. It is found also that the reduction in the repuired 3-D $\mathcal{K}$ is higher for the inside lance than that for the outside lane. For example. when $A=12 \%$, the maximum $I$ iff for the inside and outside lanes are -59.29 and $-48.16 \%$, respectively. $A$ possible reason tor this difference is that. for the inside lane, the sight line can be obstructed by the highway surface on the inside shoulder and half a lane. On the other hand, for the outside lane. the sight line can be obstructed by the inside shoulder, the inside lante, and hall : lalle. Subsequently, the potential of having a sight obstruction is higher when considering the inside lane than when considering the outside iane. The overall $3-1) \alpha$ recpuired for the highway is the maximum for both lanes. Comparing the results for different values af $A$ indicates that the 3-D $K$ is slightly lower for curves with small $A(1 \%)$. For cillves with higher $A$ (6 to $12 \%$ ), the 3-D $K$ is practically independent of the algebrate difference in grades of the vertical curve.

The effect of the other design elements on the required $3-1$ ) $K$ is shown in "Tible 7.8. The results for a higher design speed, $130 \mathrm{~km} / \mathrm{h}$. have the same trend observed for $110-\mathrm{km} / \mathrm{h}$ speed. In addition, the results suggest that the length of the horizontal curve required for the 3-D $K$ to be minimum increases as the design speed increares. Alst, the difference between 2-D and 3-D $K$ values decreases with the increase in the design speed. For example, when $A=12 \%$, Diff and the length of the horizontal curve reyuired for minimum 3-D $K$ are $-43.98 \%$ and $1100 \mathrm{~m}$, respectively, for $V=130 \mathrm{~km} / \mathrm{h}$ companed $\mathrm{kn}$. $48.16 \%$ and $700 \mathrm{~m}$ for $V=110 \mathrm{~km} / \mathrm{h}$. It is also shown in the table that, expectedly, as 
TABLE 7.8: Effect of Speed, Horizontal Curve Radius, and Superelevation Rate on 3-D K Values for Crest Vertical Curves Combined with Horizontal Curve.

\begin{tabular}{|c|c|c|c|c|c|c|c|c|c|c|}
\hline \multirow{2}{*}{ Case } & \multicolumn{2}{|c|}{$A=4 \%$} & \multicolumn{2}{|c|}{$A=6 \%$} & \multicolumn{2}{|c|}{$A=8 \%$} & \multicolumn{2}{|c|}{$A=10 \%$} & \multicolumn{2}{|c|}{$A=12 \%$} \\
\hline & $L^{\prime}$ & Diff $^{\prime}$ & $L$. & Diff & $L$ & Diff & $L_{t}$ & Diff & $L$ & Diff \\
\hline (a) & 300 & -50.83 & 300 & -48.83 & 400 & -48.61 & 600 & -48.39 & 700 & -48.16 \\
\hline (b) & 300 & -44.46 & 500 & -44.38 & 600 & -44.22 & 800 & -44.14 & 1100 & -43.98 \\
\hline (c) & 300 & -40.49 & 400 & -40.38 & 500 & -40.27 & 600 & -40.27 & 700 & -40.14 \\
\hline (d) & 300 & -62.18 & 300 & -55.73 & 400 & -55.51 & 600 & -55.39 & 700 & -55.22 \\
\hline
\end{tabular}

(a) Base case $(V=110 \mathrm{~km} / \mathrm{h}, \mathrm{SSD}=220 \mathrm{~m}, R=600 \mathrm{~m}, e=6 \%, 2-\mathrm{D} K=89.9 \mathrm{~m})$

(b) $V=130 \mathrm{~km} / \mathrm{h}(\mathrm{SSD}=260 \mathrm{~m}, R=950 \mathrm{~m}) .2-\mathrm{D} K=1255 \mathrm{~m}$

(c) $R=800 \mathrm{~m}$. $2-\mathrm{D} K=89.9 \mathrm{~m}$

(d) $e=8 \%$. 2-D $K=89.9 \mathrm{~m}$

- The length of the horizontal curve (to the nearest $100 \mathrm{~m}$ ) beyond which $L_{4}$ does not have an effect on 3-D $K$

1 The corresponding difference between the 2-D and 3-D $K$ values (Equation 7.1)

the horizontal curve radius increases, Diff decreases. For example, for $A=12 \%$, Diff changes from -48.16 to $-40.14 \%$ as $R$ increases from 600 to $800 \mathrm{~m}$. Eventually, if $R \rightarrow \infty$, the combined alignment will be a separate vertical alignment. On the other hand, as the superelevation rate increases, Diff. and in turn the effect of the 3-D alignment, increases. For example, for $A=12 \%$, Diff changes from -48.16 to $-55.22 \%$ as $e$ increases from 6 to $8 \%$. This is due to the lowering of the highway surface, which represents a potential sight obstruction relative to the sight line.

Tables 7.9 and 7.10 show the required 3-D $K$ when the development of the superelevation rate is carried out on a $90-\mathrm{m}$ transition without and with spiral curves, 
TABLE 7.9: 3-D $K$ Values for Crest Vertical Curve Combined with Horizontal Curve with $90 \mathrm{~m}$ Superelevation Runofr Iength (No Spiral Curve).

\begin{tabular}{|c|c|c|c|c|c|}
\hline \multirow{2}{*}{$\begin{array}{c}L_{\mathrm{c}} \\
(\mathrm{m})\end{array}$} & \multicolumn{5}{|c|}{$K(\mathrm{~m})$} \\
\hline & $A=4 \%$ & $A=6 \%$ & $A=8 \%$ & $A=10 \%$ & $A=12 \%$ \\
\hline \multicolumn{6}{|c|}{ Inside Lane } \\
\hline 200 & 29.1 & 94.9 & 94.9 & 94.9 & 94.8 \\
\hline 400 & 24.7 & 36.0 & 94.6 & 94.7 & 94.7 \\
\hline 600 & & & 36.2 & 9.3 .8 & 94.5 \\
\hline 800 & & & & 36.4 & 36.6 \\
\hline \multicolumn{6}{|c|}{ Outside Lane $(2-\mathrm{D} K=89.9 \mathrm{~m})$} \\
\hline 200 & 47.9 & 83.0 & 90.0 & 90.0 & 89.9 \\
\hline 400 & 44.3 & 46.0 & 79.2 & 90.0 & 89.9 \\
\hline 600 & & & 46.2 & 46.4 & 87.3 \\
\hline 800 & & & & 46.4 & 46.6 \\
\hline \multicolumn{6}{|c|}{ Both Lanes } \\
\hline 200 & 47.9 & 94.9 & 94.9 & 94.9 & 94.8 \\
\hline 400 & 44.3 & 46.0 & 94.6 & 94.7 & 94.7 \\
\hline 600 & & & 46.2 & 93.8 & 94.5 \\
\hline 800 & & & & 46.4 & 46.6 \\
\hline
\end{tabular}

Below the solid line, further increases in $L_{\mathrm{c}}$ do not change the value of $K$ 
TABIf: 7.10: 3-1) $K$ Values for Crest Vertical Curve Combined with Horizontal Curve with $90 \mathrm{~m}$ Spiral Curve at Both Ends.

\begin{tabular}{|c|c|c|c|c|c|}
\hline \multirow{2}{*}{$\begin{array}{l}L_{1} \\
\text { (m) }\end{array}$} & \multicolumn{5}{|c|}{$K(\mathrm{~m})$} \\
\hline & $A=4 \%$ & $A=6 \%$ & $A=8 \%$ & $A=10 \%$ & $A=12 \%$ \\
\hline \multicolumn{6}{|c|}{ Inside Lane } \\
\hline 200 & 29.3 & 92.6 & 92.6 & 92.7 & 92.7 \\
\hline $4(0)$ & 25.5 & 36.0 & 36.2 & 92.6 & 92.6 \\
\hline $6(x)$ & & & & 90.6 & 92.4 \\
\hline $8(X)$ & & & & 36.4 & 36.6 \\
\hline \multicolumn{6}{|c|}{ Outside Lane } \\
\hline 200$)$ & 61.7 & 87.2 & 87.3 & 89.9 & 89.9 \\
\hline 400 & 44.5 & 46.0 & 87.3 & 87.2 & 89.9 \\
\hline 600 & & & 46.2 & 86.6 & 87.3 \\
\hline 800 & & & & 46.4 & 46.6 \\
\hline \multicolumn{6}{|c|}{ Both Lanes } \\
\hline 200 & 61.7 & 92.6 & 92.6 & 92.7 & 92.7 \\
\hline 400 & 44.5 & 46.0 & 87.3 & 92.6 & 92.6 \\
\hline 600 & & & 46.2 & 90.6 & 92.4 \\
\hline 800 & & & & 46.4 & 46.6 \\
\hline
\end{tabular}

- $L_{\mathrm{c}}$ is the length of the original circular curve from point of curve to point of tangent. Actual circular curve is $L_{\mathrm{t}}-90$

- Below the solid line, further increases in $L_{4}$ do not change the value of $K$ 
respectively. Comparing Table 7.7 to Tables 7.9 and 7.10 . the effect of the development of the superelevation can be explained as follows. First. the inside lane has a $2 \%$ crossslope (normal crown-slope) which increases gradually to 6\% (full superelevationi). If the driver eye is on the transition and the object is on a fully superelevated section. or vice versa, one of them will sink relative to the other. Therefore, (he required 3-1) $k$ will increase relative to that if the cross-slope is constam. However, if the horizontal curve is long enough so that both the driver eye and the object will be on fully superelevated sections white the transition is not overlapping with the vertical curve, the superelevaltion develepment will not affect the 3-D $K$. This is illustrated by the same 3-D $K$ for long horizontal curves.

Second, the outside lane has an adverse cross-slope of $2 \%$ which changes (o) a full superelevation of $6 \%$. As a result, the lane surface will be warped and the elevations of the surface increase as the superelevation develops. Therefore, if the horizontal curve is very short, the driver's eye will be on a transition and the object will be on the other transition, and the sight line will pass over the outside lane. Therefore, the lance surface on the superelevated portion will be as a mount, and the 3-D $K$ will increase relative to that if the cross-slope is constant. As the horizontal curve length increases, the driver's eye will be on a transition while the object is on a superelevated section. or vice versa. and the sight line will pass over the inside lane. As a result, the elfective height of the driver's eye, or the object, will increase and the required 3-D $K$ will decrease. However. the portion of the vertical curve that is not overlapping with the horizontal curve will produce a greater 3-D $K$ that controls the curve. As a result, the 3-D $K$ will be the same 
like that for separate vertical alignment and for combined alignment with constant crossslope. Finally, as the entire vertical curve is overlapping with the horizontal curve, the fully superelevated portion will control the 3-D $K$ and the result will be identical to that in the case of a constant cross-slope.

\subsection{Summary}

It is shown in this chapter that the analytical model for 3-D sight distance can be very usceful in establishing geometric design standards based on 3-D combined highway alignment. The model is used to design combined horizontal and vertical curves with the sight distance as the main controlling parameter. It is shown that the 2-D and 3-D designs may differ significantly with the result that the current 2-D-based design standards are compromising traffic safety or highway economy. The model is also used to study the effect of the different design elements on the 3-D design. It is shown that both the $r_{-i} \quad \mathbb{R}$ for horizontal curves and $\boldsymbol{X}$ for crest vertical curves depend on all other design elements (superelevation rate, SSD, side-slope, deflection angle and degree of horizontal curves, flatness of vertical curves, and degree of overlap between horizontal and vertical curves). lgnoring the interaction among these elements may lead to erroneous results.

The addition of down-slope and/or flat drainage ditch before the up-slope in cut sections reduces the impact of the 3-D sight distance on the design elements, and, intuitively, reduces the effect of aligament overlap on the 3-D sight distance. This explains in part ihe small difference between the marking of passing and no-passing zones using the 2-D and 3-D sight distances (Chapter 6). On the segment used in the marking application, cuwn-slopes, with or without flat ditches, are always added before the up- 
slopes in the cut sections. and thus the effect of the alignment overlap is relatively reduced. It should be noted, however, that sometimes the topography may not allow such provisions. For example, Figure 7.3 shows a horizontal curve combined with a crest vertical curve on Trans-Canada Highway (Highway 17). As shown in the figure. an upslope is introduced immediately after the shoulder with no provision for down-slope or ditch.

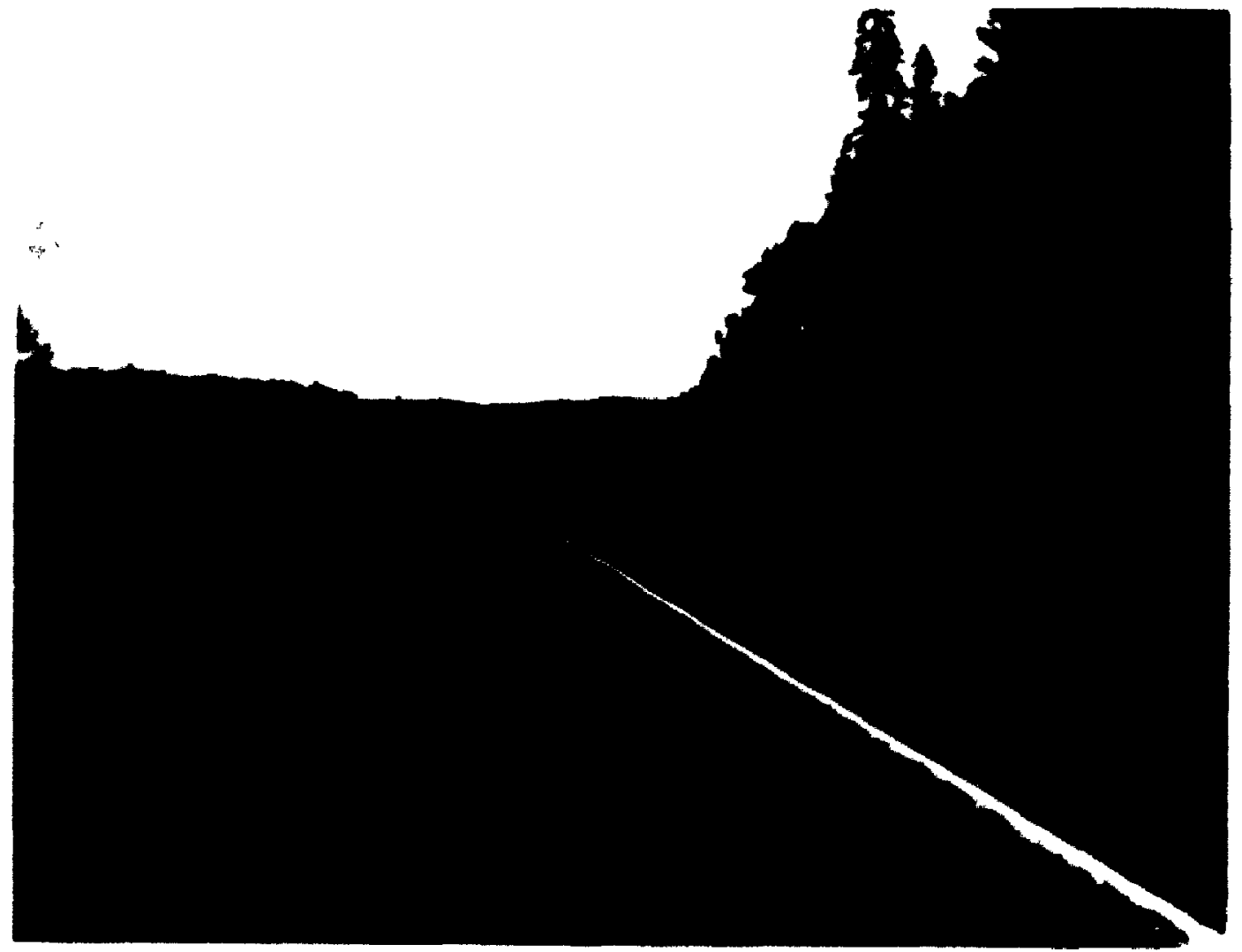

FIGURE 7.3: Combined Alignment on Cut Section on Highway 17. 


\section{CHAPTER 8}

\section{CONCLUSIONS AND RECOMMENDATIONS}

\subsection{Summary}

As has been demonstrated throughout this research, highway geometric design is a vital phase in the design of safe, pleasing, and efficient highways. However, the develor ment of the current highway geometric design standards contains a number of rough assumptions, and many new rescarch findings have not been adopted in the several revisions of the standards over the last forty years. Among these rough assumptions is ignoring the 3-D nature of highway alignment. Such an assumption has been introduced because of the anticipated difficulties associated with considering the 3-D interaction among the different design elements. As a result, the design standards can be inaccurate. and the resulting margin of safety or hazard cannot be estimated.

In this research, the subject of sight distance on rural highways is addressed comprehensively. Although sight distance is agreeably believed to be an important element in highway safety and efficiency, not much work has been done to determine the required or the available sigint distance accurately. Even though, most of the work done has not been considered in the design standards. Therefore, the research presents a complete study of the required passing sight distance and other requirements for safe and efficient passing manocuvres on two-lane highways (length, beginning, and end of passing zones). The sight distance required for stopping is being studied in the NCHRP Project 3-42 and is beyond the scope of this research. 
In addition to the requirements for passing manceuvres. the rescarch presents comprehensive analytical models for available daytimxe sight distance on complex separate 2-D horizontal and vertical alignments. These models fill the gaps that currenty exist in this area where the current models can deal only with smple and isolated horizomal or vertical curves. The developed models are coded into compuler programs w delermine the profile of available sight distance on complex 2-D alignments at a user-specified accuracy. Furthermore, an unprecedented analytical morlel wevaluate the avaliable daytime sight distance in 3-D combined alignments is developed. The analyticial moxlel can be considered an application of the finite element technique in highway geometric design. The modelling proved to be very accurate, especially for straight seguncmts and parabolic vertical curves where the modelling is axact. For circular and spiral horiantal curves and vertical spline grades. the modelling is approximate, and the smaller the element size used in the modelling the higher the accuracy. The mokled is alsocoded into a computer program that can determine the profile of available sight distance on 3-1) complex alignments at a user-specified accuracy.

Finally, two applications for the developed models are presented: namely marking of passing and no-passing zones on two-lane highways and design of $3-1)$ combined alignments. In the marking application, the 2-D and 3-D computer programs and inc developed requirements for passing are used to determine the marking of passing and mopassing zones on a $7-\mathrm{km}$ segment of Highway 61 . In the design application. the minimum required radius of horizontal curves, $R$, and the minimum length of vertical crest curves, $L$, are determined in 3-D combined alignments. These 3-D values of $K$ and 
$L$ are compared with the corresponding 2-D values according to the current standards. Thus, the effeci of ignoring the 3-D interaction among the different highway design elements is quantified.

\subsection{Conclusions}

First, the literature review presented in Chapter 2 shows a number of deficiencies in the current highway geometric design standards. Among these deficiencies is the design of highway alignments in 2-D projections separalely from the other elements. Even in this approximation, the design standards can deal only with a simple and isolated element at a time, e.g.. a simple circular curve with long tangents and with a curve length greater than the required sight distance. Expectedly, the resulting formulas are simple but of limited value in practical applications (AASHTO, 1990; 1994).

Second, several discrepancies are encountered in the current design and marking standards regarding the requirements for passing manoeuvres on two-lane highways. Furthermore, the design standards are not free from self-discrepancies while the reasons behind the values recommended in marking standards are not clear. Other models have been developed to provide more accurate and realistic modelling for the passing manocuvres. However, close investigations of these models show that revisions are still needed. Moreover, all of these models have focused on the minimum required passing sight distance, and none of them has investigated the minimum length of passing zones or the optimum beginning and end of passing zones. A revised analytical model is presented in Chapter 3 to overcome these setbacks. The model provides a comprenensive 
modelling for the entire passing manocuvre from its beginning to end. The resulting values of minimum required passing sight distance have been validalted with field measurements, and they have showed superiority over the other existing models. A comparison with the current marking standards shows that these standards can be unsalfe. The degree of hazard that may result from the marking standards increases with the increase of the design speed where the consequences of a collision are more severe.

Third, the analytical models and computer software for 2-D and 3-1) sight distances, presented in Chapters 4 and 5, have proven to be accurate and comprehensive through graphical and field verifications. The software can consider virtually all possible types of sight obstructions; namely, highway surface, continuous latteral obstructions. single lateral obsttu :ions, and overpasses. Moreover, the single lateral obstructions can be defined by one, two, or three points, and the overpasses can have variable vertical clearance and/or can be at skewed angle with the highway centerline. These models and software provide a very useful tool for marking passing and no-passing zoncs on Iwo-lanc highways and for designing combined alignments in 3-D projections.

Fourth, a marking application for a 7-km segment of llighway ol is used in Chapter 6 to show the applicability of the developed 2-D and 3-1) software. The sofiware can determine the profile of available 2-D and 3-D sight distances using the aligninent and cross-section data that are already available in the highway agencies. The maximum element size specified in the alignment modelling can affect the computer run-time and the modelling accuracy. The optimum size depends on the irregularities of the alignment and cross-section data. However, a maximum element size as large ar, 25 in dres not 
compromise the modelling accuracy in this application example. Interestingly, the modelling accuracy of the 3-D combined alignment is relatively insensitive to the maximum element size up to $50-\mathrm{m}$ maximum element size. The profiles of available sight distance can be used for marking passing and no-passing zones according to the MUTCD standards or according to the requirements developed in this research. A comparison between the two methods of marking shows that the MUTCD standards can compromise the traffic safety by allowing passing on unsafe sections, and can compromise the highway level-of-service by disallowing passing on safe sections. The marking on the field is, however, more conservative but on the expense of the highway quality of service.

The profiles of 2-D and 3-D sight distances can differ significantly. The 2-D separate alignment tends to overestimate the available sight distance when a crest vertical curve overlaps with a horizontal curve in a cut section. On the other hand, the 2-D separate alignment tends to underestimate the available sight distance when a sag vertical curve overlaps with a horizontal curve in a cut section or when a crest vertical curve overlaps with a horizontal curve in a fill section. However, this difference does not translate into significant difference in the marking in this specific application. The reasons behind this are: (1) the 2-D and 3-D sight distances differ from each other along short distances while the sight distance for passing is required on long distances, (2) the 2-D and 3-D sight distances differ from each other on obstructed segments where the available sight distance is less than the required sight distance, and (3) the provision of 
down-slopes and flat ditches before the up-slopes in cut sections reduces the effect of the combined alignment on the sight distance.

Finally, Chapter 7 presents an application for the use of the 3-D model and software in designing combined highway alignments. Because the design is based on the stopping sight distance which is considerably less than the passing sight distance. the differences between the 2-D and 3-D sight distances have a significant effice on the highway design. When a horizontal curve is overlapping with a crest curve in a cut section, the required radius, $R$, can be significantly higher than the 2-D value recommended in the design standards (up to 130\%). On the other hand, the required $R$ can be significantly lower than the 2-D value recommended in the design standiards (no curve may be required for small deflection angles) when the horizontal curve is overlapping with a sag curve in a cut section. Also, when a crest vertical curve is overlapping with a horizontal curve in a fill section, the required length of the vertical curve, $L$, can be significantly lower than the 2-D value recommended in the design standards (up to 50\%). It is also shown that the highway design elements: namely $R$ and $L$ depend on the values selected for the other design elements such as, deflection angle of horizontal curve, degree of alignment overlap, superelevation ratc, required stopping sight distance, side-slope, and superelevation development.

\subsection{Recommendations and Future Research}

Based on the findings of this research, presented in the previous chapters and summarized above, the following can be recommended: 
- The current standards for marking passing and no-passing zones on two-lane highways require major revisions to eliminate the discrepancies related to the passing manoeuvre and to ensure that the resulting marking can effectively assist drivers take a decision to pass or trail a slower vehicle without compromising the highway's level-of-service. The revised model presented in this research can provide a useful tool in such revisions.

- The current design standards require major revisions to enhance the design requirements based on 3-D combined alignments and to establish 3-D design standards. The analytical model and software developed in this research can provide a useful tool in the consideration of the daytime sight distance. However, further research is still needed for establishing a complete set of 3-D design standards.

- Designers should be aware of the effect of the combined alignment on the sight distance provisions. Ignoring this effect may lead to constructing roads with too liberal and too conservative sections, even though all of them are designed according to the current standards. In modifying existing roads, ignoring the interaction among the different highway design elements may lead to erroneous allocation of financial resources where safe sections may be modified and unsafe sections are overlooked.

- Highway agencies should survey the single lateral obstructions in addition to the horizontal and vertical alignments and cross-section data.

Because this research has focused mainly on daytime sight distance on rural highways, further research is still needed and can be very fruitful as follows: 
- An accurate consideration of the nighttime sight distance (headlight sight distance) in highway design has not been modelled. At nighttime, drivers can see just as far as their vehicles' headlights cover. Although the design standards include provisions for designing sag vertical curves based on the headlight sight distance, only 2-D simple isolated sag is considered. Analytical models for 2-D and 3-D headlight sight distance are still lacking.

- The revised model for required PSD, minimum length of passing zoncs, and beginning and end of passing zones developed in this research was deterministic. That is a specific design value was assume to each parameter in the model. However, due to the wide variations in drivers' reactions and performances in the same situalion, a probabilistic analysis should be carried out. Such an analysis would determine the required PSD, minimum length of passing zones, and beginning and end of passing zones that would be sufficient for a specific percentage of drivers and vehicles on each specific highway.

- In this research as in all current models, the driver of the passing velicle is assumed to have a clear sight distance that can be obstructed by the alignment only. However. in reality, the impeding vehicle is by default ahead of the passing vehicle and may fall on the sight line of the passing driver. Therefore, the impeding vehicle may act as a moving obstruction that may block the passing driver's sight distance. Such possibility needs to be investigated closely to simulate the passing manoeuvre as accuralc an possible. 
- The developed models for 2-D and 3-D sight distance can be enhanced to consider the intersection sight distance. Since urban intersections are usually controlled, and speeds are relatively low, the intersection areas are usually small enough to be approximated into a 2-D plan. However, rural intersections are usually uncontrolled, and speeds are relatively high. Subsequently, approximating the intersection into a 2-D plan may lead to erroneous results. 


\section{REFERENCES}

AASHO. 1954. A Policy on Geometric Design of Rural Highways. American

Association of State Highway Officials. Washington. D.C.

AASHO. 1940. A Policy on Sight Distance for Highways. Anxerican Assonciation of State Highway Officials, Washington, D.C.

AASHTO. 1994. A Policy on Geometric Design of Highn'ans and Strenets. American Association of State Highway and Transportation Officials, Washington, I).C. AASHTO. 1993. Highway Drainage Guidelines. Vols. 1-11. American Assceialton af State Highway and Transportation Officials, Washington. D.C.

AASHTO. 1991. Model Drainage Mamual. American Association of State llighway and Transportation Officials, Washington, D.C.

AASHTO. 1990. A Policy on Geometric Design of Highways and Streders. Americal! Association of State Highway and Transportation Officials, Washington, D.C. AASHTO. 1986. AASHTO Guide for Design of Pavement Siruchures. American Association of State Highway and Transportation Officials, Washington, I).(

Adler, H.A. 1987. Economic Appraisal of Transport Projects. Published for the Economic Development Institute of the World Bank, The Jhons Ilopkins University Press, Baltimore, U.S.A.

Agg, T.R. 1916. Construction of Roads and Pavements. First Edition. (Ciled in Hall and Turner, 1989) 
Allen, R.W., T.J. Rosenthal, and D.H. Klyde. 1995. Application of Vehicle Dynamic Modeling to Interactive Highway Safety Design. Presented at the Transportation Research Board Annual Meeting, January 22-27.

ASCE. 1977. Practical Highway Aesthetics. American Society of Civil Engineering, New York. N.Y.

Berg, W.D., J. Choi, and E.J. Kuipers. 1989. Development of Highway Alignment Information from Photolog Data. Transportation Research Record 1239, TRB, National Research Council, Washington, D.C., pp. 54-61.

Blanchard. A.H., and H.B. Drowne. 1914. Text-Book on Highway Engineering. First Edition. (Cited in Hall and Turner, 1989)

BTS. 1996. National Transportation Statistics. Bureau of Transportation Statistics, U.S. Departmert of Transportation, Washington, D.C.

Cook, R.D., D.S. Malkus, M.E. Plesha. 1989. Concepts and Applications of Finite Element Analysis. Third Edition, John Wiley \& Sons, Inc., New York, N.Y.

Dräbek, K. 1969. Plane Curves and Constructions. Chapter 4: Survey of Applicable Mathematics. Edited by K. Rektoıvs, M.I.T. Press, Cambridge, Mass.

Dunlap, D.F., P.S. Fancher, R.E. Scott, C.C. MacAdam, and L. Segel. 1978. Influence of Combined Highway Grade and Horizontal Alignment on Skidding. National Cooperative Highway Research Program, NCHRP Report 184, TRB National Research Council. Washington, D.C.

Easa. S.M. 1995. Geometric Design. Chapter 60: Civil Engineering Handbook. Edited by W.F. Chen. CRC Press. Boca Raton, Fla. 
Easa, S.M. 1994a. Design Considerations for Highway Reverse Curves. Transportation Research Record 1445. TRB, National Research Council. Washington. D.C.. pp. 1-11.

Easa, S.M. 1994b. Design Considerations for Highway Sight-Hidden Dips. Journal of Transportation Research, Vol. 28A. No. 1, pp. 17-29.

Easa, S.M. 1993. Lateral Clearance Needs on Compound Horizontal Curves. Joumal of Transportation Engineering, ASCE, Vol. 119, No. 1, pp. 111-123.

Easa, S.M. 1992. Sight Distance Relationships for Symmetrical Say Curve with Noncentered Overpass. Journal of Transportation Research, Vol. 26B. No. 3. pp. 241-251.

Easa. S.M. 1991a. Lateral Clearance to Vision Obstacles on Horizontal Curves. Transportation Research Record 1303, TRB, National Research Council, Washington, D.C., pp. 22-32.

Easa, S.M. 1991b. Sight Distance Model for Unsymmetrical Crest Curves.

Transportation Research Record 1303, TRB, National Research Council, Washington, D.C., pp. 39-50.

Easa, S.M. 1991c. Sight Distance Model for Unsymmetrical Sag Curves.

Transportation Research Record 1303, TRB, National Research Council. Washington, D.C., pp. 51-62.

Fambro, D.B., K. Fitzpatrick, and R. Koppa. 1995. A New Stopping Sight Distance Model for Use in Highway Geometric Design. Presented at the Internatiomal 
Symposium on Highway Geometric Design Practices. Boston, Massachusetts, USA, August 30-September 1.

FHWA. 1995. Status Report: Strategic Highway Research Program Product Implementation. Federal Highway Adminstration, U.S. Department of Transportation, Washington, D.C.

FHWA. 1972. Eicomemic and Social Effects of Highways. Federal Highway Adminstration, U.S. Department of Transportation, Washington, D.C.

Fitzpatrick, K. 1994. Horizontal Curve design: An Exercise in Comfort and Appearance. Transportution Research Record 1445, TRB, National Research Council, Washıngton, D.C., pp. 47-53.

French, A. 1982. Vehicle Operating Characteristics. Chapter 6 (B): Transportation and Truffic Engineering Handbook. Edited by S.H. Wolfgang, L.E. Keefer, and W.R. McGrath, Institute of Transportation Engineers, ITE, Second Edition, Prentice-Hall, Inc., Englewood Cliffs, New Jersey.

Gallaway, B.M., R.E. Schiller, and J.D. Rose. 1971. The Effect of Rainfall Intensity, Pavement Cross Slope, Surface Texture, and Drainage Length on Pavement Water Depths. Research Report No. 138-5, Texas Transportation Institute. (Cited in Dunlap et al, 1978)

Garher, J.G., and L.A. Hoel. 1988. Traffic and Highway Engineering. West Publishing Company, St. Paul, MN.

Geissler, E.H. 1968. A Three-Dimensıonal Approach to Highway Alignment Design. Highway Resedrch Record 232, HRB, National Research Council, Washington, 
D.C.. pp. 17-28. (Also published as Report No. RR138, Minisiry of

Transportation of Ontario, MTO. Downsview. Ontario, 1968.)

Glauz, W.D., D.W. Harwood, and A.D. St. John. 1980. Projected Vehicle

Characteristics Through 1995. Transportation Re'se'ure'h Record 772. TRB.

National Research Council. Washington, D.C., pp. 37-44.

Glennon, J.C. 1989. Highway Sight Distance Design Issues: An Overview.

Transportation Research Record 1208, TRB. National Rescarch Council.

Washington, D.C.. pp. 1-3.

Glennon, J.C. 1988. New and Improved Model of Passing Sight Distance on Two-

Lane Highways. Transportation Research Record I195. TRB. National

Research Council, Washington, D.C., pp. 1.32-137.

Glennon, J.C. 1987. Effect of Sight Distance on Highway Salety. Transpurrtation

Research Board, State of the Art Report 6, National Research Council.

Washington, D.C., pp. 65-77.

Glennon, J.C., and G.D. Weaver. 1972. Highway Curve Design for Salie Vehicle Operations. Highway Research Record 390. HRB, National Research Council, Washington, D.C., pp. 15-26.

Good, D., J.B.L. Robinson, G. Sparks, and R. Neudrol. 1991. The Liffect of Velhicle Length on Traffic on Canadian Two-Lane, Two-Wav Resads. Transportation Association of Canaua. Ottawa. Ontario.

Goult, K.J. R.F. Hoskins, J.A. Milner, and M.J. Prall. 1973. Applicahle Mathematics: A Course for Scientists and Engineers. The Macmillan Press LAd, I andon, lingland. 
Guillet, E.C. 1966. The Story of Canadian Roads. University of Toronto Press, Toronto, Canada.

Hall, J.W., and D.\$. Turner. 1989. Stopping Sight Distance: Can We See Where We Now Stand? Transportation Research Record 1208, TRB, National Research Council, Washing!on, D.C., pp. 4-13.

Hiarwood D.W., and J.M. Mason. 1994. Horizontal Curve Design for Passenger Cars and Truck Iransportation Research Record 1445. TRB, National Research Council. Washington. D.C., pp. 22-33.

Harwoxd. D.W.. and J.C. Glennon. 1989. Passing Sight Distance for Passenger Cars and Trucks. Transportation Research Record 1208. TRB, National Research Council, Washington, D.C., pp. 59-69.

Harwood. D.W.. and J.C. Gilennon. 1977. Framework for Design and Operation of Passing \%ones on Two-Lane Highways. Transportation Research Record 601, TRB, National Research Council. Washington. D.C.. pp. 45-50.

Hassan. Y., S.M. Easa, and A.O. Abd El Halim. 1996a. Passing Sight Distance on Two-Lane Highways: Review and Revision, Journal of Transportation Researih (in press).

Hatssan, Y.. S.M. Easa, and A.O. Abd El Halim. 1996b. Analytical Model for Sight Distance Analysis on 3-D Highway Alignments. Transportution Research Record 1523. TRB. National Research Council, Washington, D.C., pp. 1-10. Hassan. Y.. S.M. Easa, and A.O. Abd El Halim. 1996c. Computer Model for Establishing No-Passing Zones on 3-D Highway Alignments. Proceedings, Ist 
Transportation Speciality Conference for the Canadian Soxiety for Civil Engineering. CSCE. Edmonton. Alberta. May 29-Junc I. Vol. Illb, pp. \$11422.

Hassan, Y., S.M. Easa, and A.O. Abd El Halim. I006d. Toward 3-D Highway Geometric Design Standards. Proceedings. I.st Tramspontation Sperciality Comference for the Camadian Societs for Civil Engmeering. CSCI: Edmonton. Alberta, May 29-June 1, Vol. IIIh, pp. 345-354.

Hassan, Y., S.M. Easa, 1.O. Abd El Halim. 1996e. Design Comsiderations for Combined Highway Alignments. Accepted for Publication in the Jomrmal of Transportation Engineering, ASCE.

Hassan, Y., A.O. Abd El Halim, and S.M. Easa. 1995a. Design Considerations forf Passing Sight Distance and Passing Zones. Presented dt the Into'matiomal Symposium on Highna Geometric Design Practices. August 30). Septeanter 1, Boston, Massachusetts, USA.

Hassan, Y., S.M. Easa, and A.O. Abd El Halim. 1995h. Sight Distance of Hlorizulal Alignments with Continuous Lateral Obstructions. Tramspormation Kesearch Record 1504, TRB, National Research Council, Washington I).C., pp. 3]-42.

Hassan. Y., S.M. Easa, and A.O. Abd El Halim. I995c. New Development for Establishing No-Passing Zones on Two-I ane Highways due lo Verikal Alignments. Proceedings, Annual Comference of the Canadian Sincielv for ( sid Engineering, CSCE, Ottawa, Vol. IV, pp. 205-214. 
Hassan, Y., S.M. Easa, and A.O. Abd El Halim. 1995d. New Methodology for Available Sight Distance in Two-Dimensional Horizontal and Vertical Alignments, Proceedings, Al-Azhar Engineering Fourth International Conference. Cairn, Egypt, December 16-19, 1995, Vol. 4, pp. 283-294.

Hassan, Y., S.M. Easa, and A.O. Abd El Halim. 1995e. User's Guide of MARKC: A Program for Establishing No-Passing Zones on Two-Lane Highways due to Combined Horizontal and Vertical Alignments. Research Report LU-TRC-RR95-1. Transportation Research Centre, Lakehead University, Thunder Bay, Ontario.

Hassan, Y., S.M. Easa, and A.O. Abd El Halim. 1994a. User's Guide of MARKV: A Program for Establishing No-Passing Zones on Two-Lane Highways due to Vertical Alignment. Research Report LU-TRC-RR-94-3, Transportation Research Center, Lakehead University, Thunder Bay, Ontario.

Hassan, Y., S.M. Easa, and A.O. Abd El Halim. 1994b. User's Guide of MARKH: A Program for Establishing No-Passing Zones on Two-Lane Highways due to Horizontal Alignment. Research Report LU-TRC-RR-94-1, Transportation Research Center, Lakehead University, Thunder Bay, Ontario.

HCM. 1994. Highway Capacity Manual. Special Report 209. Transportation Research Board, National Research Council, Washington, D.C.

Huang. Y.H. 1993. Pavement Analysis and Design. Preritice-Hall, Inc., Englewood Cliffs, New Jersey. 
Jones, J.R. 1970. An Evaluation of the Sufety and Utilization of Short Passing Sections. Texas A\&M University, College Station, MS Thesis. (Cited in Harwood and Glennon, 1977)

Jull, D.E., and G.T. Murray. 1984. ITEDS: A Computer Aided Design System for Highway Engineering. Proceedings, Annual Conference of the Camadian Siociety. for Civil Engineer: 1 g, CSCE. Halifax, May 23-25. pp. 431-445.

Khisty, C.J. 1990. Transportation Engineering: An Introduction. Prentice-Hall, Inc.. Englewood Cliffs, New Jersey.

King, C.L., and W.B. Harkins. 1982. Geometric Design. Chapter 19: Transportaniom and Traffic Engineering Handbook. Edited by S.H. Uolfgang. L.E. Keefer. and W.R. McGrath, Institute of Transportation Engineers, ITE, Second Edition, Prentice-Hall, Inc., Englewood Cliffs, New Jersey.

Kontaratos, M., B. Psarianos, and A. Yotis. 1994. Minimum Hori־ontal Curve Radius as Function of Grade Incurred by Vehicle Motion in Driving Morle. Transportation Research Record 1445, TRB, National Research Council, Washington, D.C., pp. 86-93.

Kosasih, D., R. Robinson, and J. Snell. 19:97. A Review of Some Recent Geometric Road Standards and Their Application to Developing Countries. Transport asıd Road Research Laboratory, TRRL, Research Report 114. England.

Krammes, R.A., and M.A. Garnham. 1995. Review of Alignment Design Policies Worldwide. Presented at the International Symposium on Highway (ieomn Iric Design Practices, Boston, Massachusetts, USA, August 30-September I. 
Lamm, R. 1984. Driving Dynamic Consideration: A Comparison of German and American Friction Coefficients for Highway Friction. Transpori Atıon Research Record 960, TRB, National Research Council, Washington, D.C., pp. 13-20.

Lamm, R., and B.L. Smith. 1994. Procedure for Detecting Errors in Alignment Design and Consequences for Safer Redesign. Transportation Research Record 1445, TRB, National Research Council, Washington, D.C., pp. 64-72.

Larson, T.R. 1996. Application of 3D and 4D Visual Modeling for Transportation Systems (NCHRP Project 20-5). Presented at the Transportation Research Board Annual Meeting, January 7-11.

Lieberman, E.B. 1982. Model for Calculating Safe Passing Sight Distances on TwoLane Rural Roads. Transportation Research Record 869, TRB, National Research Council, Washington, D.C., pp. 70-76.

Mannering, F.L., and W.P. Kilareski. 1990. Principles of Highway Engineering and Traffi Analysis. John Wiley \& Sons, Inc. New York, N.Y.

Meijer, F., and O. Van Nijf. 1992. Trade, Transportation and Society in Ancient World. Routledge, Chapman and Hall Inc., New York, N.Y.

Michael, H.L. 1960. Highway Planning. Section 2: Highway Engineering Handbook. Edited by K.B. Woods, D.S. Berry, and W.H. Goetz, McGraw-Hill, Inc., New York, N.Y.

Mori. Y.. M. Kurihara, A. Hayama, S. Ohkuma. 1995. A Study to Improve the Safety of Expressways by Desirable Combinations of Geometric Alignments. 
Presented at the International Simposium on Highway Geometric Design

Practices, Boston, Massachusetts, USA, August 30-September 1.

MUTCD. 1995. Manual of Uniform Traffic Control Devices. Ministry of Tran: ,ortation of Ontario, MTO, Downsview, Ontario.

MUTCD. 1988. Manual of Uniform Traffic Control Devices. Federal Highway Administration, FHWA, Washington, D.C.

MUTCD. 1976. Manual of Uniform Traffic Control Devices for Canuda.

Transportation Association of Canada, TAC, Ottawa, Ontario.

NAASRA 1980. Interim Guide to the Geometric Design of Rural Ronds. National Association of Australian State Road Authorities., Sydney, Australia.

Neuman, T.R. 1989. New Approach to Design for Stopping Sight Distance.

Transportation Research Record 1208. TRB. National Research Council, Washington, D.C., pp. 14-22.

Neuman, T.R., and J.C. Glennon. 1984. Cost-Effectiveness of Improvements to Stopping Sight Distance. Transportation Research Record 923, TRB, National Research Council, Washington, D.C.. pp. 26-34.

Noble, C.M. 1960. Geometric Design. Section 22: Highway Engincering Ilandhouk. Edited by K.B. Woods, D.S. Berry, and W.H. Goetz, McGraw-Hill, Inc., New York, N.Y.

Oglesby, C.H., and R.G. Hicks. 1982. Highway Engineering. Fourth Edition, John Wiley \& Sons, Inc., New York, N.Y 
Olson, P.L., D.E. Cleveland, P.S. Fancher, L.P. Koystyniuk, and L.W. Schneider. 1984. Parameters Affecting Stopping Sight Distance. National Cooperative Highway Research Program, NCHRP Report 270, TRB, National Research Council, Washington, D.C.

Park. R.A., N.J. Rowan, and N.E. Walton. 1968. A Computer Technique for Perspective Plotting of Roadways. Highway Research Record 232, HRB, National Research Council, Washington, D.C., pp. 29-45.

PCA. 1984. Thickness Design for Concrete Highway and Street Pavements. Portland Cement Association, Chicago, Illinois.

Persaud, B.N. 1992. Roadway Safety - A Review of the Ontario Experience and Relevant Work Elsewhere. Report PAV-92-02, Ministry of Transportation of Ontario, Downsview, Ontario.

Reagan, J.A. 1994. The Interactive Highway Safety Design Model: Designing for Safety by Analyzing Road Geometrics. Public Roads, Vol. 58, No. 1, pp. 3743.

Reagan, S. 1995. Influence of Road Geometrics on Vehicle Dynamics. Presented at the International Symposium on Highway Geometric Design Practices, Boston, Massachusetts, USA, August 30-September 1.

Rıllet, L.R., B.G. Hutchinson, and M. Whitney. 1990. Mechanics of the Passing Maneuver and the Impact of Large Trucks. Journal of Transportation Research, Vol. 24A. No. 2. pp. 121-128. 
Ross, N.F., and K. Russam. 1968. The depth of Rain Water on Road surfaces. Road and Research Laboratory, RRL. Report No. LR 236, England. (Cited in Dunlap et al, 1978)

Saito, M. 1984. Evaluation of the Adequacy of the MUTCD Minimum Passing Sight Distance Requirement for Aborting the Passing Maneuver. Journal of the Institute of Transportation Engineers, pp. 18-22.

Sanchez, E. 1994. A 3-Dimensional Analysis of Sight Distance on Interchange Connectors. Transportation Research Record 1445, TRB, National Research Council, Washington, D.C., pp. 101-108

Smith, B.L., and R. Lamm. 1994. Coordination of Horizontal and Vertical Alignment with Regard to Highway Aesthetics. Transportation Research Recourd 1445. TRB, National Research Council, Washington, D.C., pp. 7.3-8.5.

Sparks, G.A., R.D. Neudorf, J.B.L. Rohinson, and D. Good. 1993. Effects of Vehicle Length on Passing Operations. Journal of Transporfation Engine'ering. AS( I:, Vol. 119, No. 2, pp. 272-283.

Staughton, G.C.. and T. Williams. 1970. Tyre Performance in Wet Surface Comditioms. Road and Research Laboratory, RRL, Report No. IR 355, England. (Cited in Dunlap et al, 1978)

TAC. 1990. Canada's Roadway Infrastructure: Selected Facts and Figures. Transportation Association of Canada, Ottawa, Ontario.

TAC. 1986. Manual of Geometric Design Standards for Canadian Rouds. Transportation Association of Canada, Ottawa, Ontario. 
Tanton, M.S., R.M. Prescott, N. Walji, W.B. Currie. D.R. Saunders, R.G. Charlwood, P.Salt, C.W. Baker, and S. Gray. 1986. The Use of Computer-Aided Design Techniques in Highway Engineering. Transportation Association of Canada, TAC, Ottawa, Ontario.

TRB. 1987. Designing Safer Roads: Practices for Resurfacing, Restoration, and Rehubilitation. Special Report 214. Transportation Research Board, National Research Council, Washington, D.C.

Van Der Tak, H.G., and A. Ray. 1971. The Economic Benefits of Road Transport Projects. Occasional Paper No. 13, World Bank.

Van Valkenberg. G.W., and H.L. Michael. 1971. Criteria for No-Passing Zones. Highway Research Record 366, HRB, National Research Council, Washington, D.C., pp. 1-9.

Waissi, G.R., and D.E. Cleveland. 1987. Sight Distance Relationships Involving Horizontal Curves. Transportation Research Record 1122, TRB, National Research Council, Washington, D.C., pp. 96-107.

Weaver. G.D., and J.C. Glennon. 1971. Passing Performance Measurements Related to Sight Distance Design. Research Report 134-6. Texas Transportation Institute. Wrighı, P.H., and R.J. Paquette. 1979. Highway Engineering. Fourth Edition, John Wiley \& Sons, Inc.. New York, N.Y.

Yeager, R.W. 1971. The Depth of Rain Water on Road Surfaces. Unpublished Report, Goodyear Tire \& Rubber Co. (Cited in Dunlap et al, 1978) 
25.3

Yeager, R.W., and J.D. Miller. 1971. Actual Water Depths on Roadways armed Its Relationship to Tire Testing. Unpublished Report, Goodyear Tire \& Rubber Co. (Cited in Dunlap et al. 1978)

Yoder. E.J., and M.W. Witczak. 1975. Principles of Pavement Design. John Wiley \& Sons, Inc., New York. N.Y.

Young, J.C., R.D. Miles, and C.L. Miller. 1960. Route Selection, Airpholo

Interpolation. Photogrammetry, and Digital Computers. Section S: Highway Engineering Handbook. Edited by K.B. Woods, D.S. Berry, and W.II. Ci(x-1y., McGraw-Hill, Inc., New York. N.Y.

Zienkiewicz, O.C., and R.L. Taylor. 1991. The Finite Element Method. Fourth Edition. McGraw-Hill Book Company Limited, London, England. 

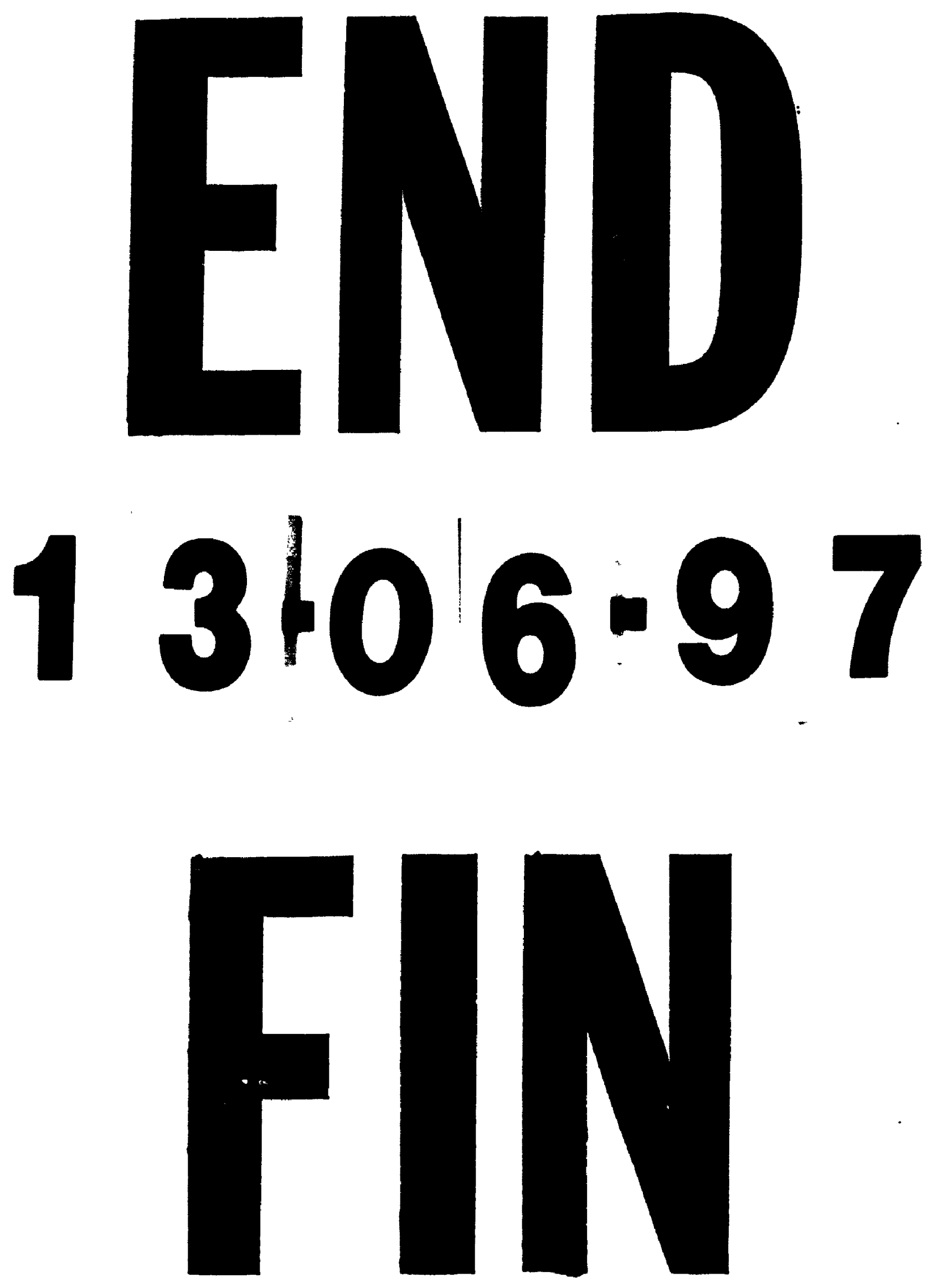